\title{
DIE LOKALE STRUKTUR VON T-DUALITÄTSTRIPELN
}

\author{
Dissertation zur Erlangung des Doktorgrades \\ der Mathematisch-Naturwissenschaftlichen Fakultäten \\ der Georg-August-Universität Göttingen \\ angefertigt von \\ ANSGAR SCHNEIDER \\ aus \\ FOERDE \\ geboren in \\ SIEGEN
}

Göttingen, im Oktober 2007 
D7

Referrent: Prof. Dr. Ulrich Bunke

Koreferent: Prof. Dr. Thomas Schick

Tag der mündlichen Prüfung: 5. 11. 2007 
Meinen Eltern und Großeltern

- Jetzt habt ihr den Salat! 



\section{Vorrede und Danksagung}

Die vorliegende Arbeit ist das wesentliche Ergebnis meiner Zeit am mathematischen Institut der Universität Göttingen. Dort war ich vom Sommer 2005 bis zum Herbst 2007 Promotionsstudent, unterstützt durch den dortigen Graduiertenkolleg Gruppen und Geometrie.

Wie der Titel sagt, beschäftigt sich die Arbeit mit der lokalen Struktur von T-Dualitätstripeln. T-Dualitätstripel (das $\mathrm{T}$ steht für Torus) sind mathematische Objekte, die sich als zweckmäßig erwiesen haben, T-Dualität mittels topologischer Methoden zu beschreiben [BRS]. T-Dualität selbst ist eine Dualität von Stringtheorien [Po] auf zwei verschiedenen Raumzeitmanigfaltigkeiten. Im einfachsten Falle sind diese durch eine Transformation Radius $\mapsto 1 /$ Radius entlang einer kompaktifizierten Raumdimension (einem Torus) miteinander identifiziert. Das Verhalten der zugrundeliegenden Felder und die Geometrie und Topologie der Raumzeitmanigfaltigkeiten unter dem Übergang von einer Raumzeit zu der anderen ist das, was durch T-Dualität beschrieben wird. Die Literatur zu diesem Thema ist reichhaltig, und man möge etwa [BEM, BHM1, $M R, B R S]$ und der darin zitierten Literatur folgen, um einen Überblick darüber zu erhalten. Neben der schon erwähnten topologischen Beschreibungsweise, ist in [MR] ein $C^{*}$-algebraischer Zugang beschrieben, dessen zentrale Objekte gewisse $C^{*}$-dynamische Systeme sind, deren Dualitätstheorie wiederum eine andere mathematische Beschreibung von T-Dualität liefert.

Das Ziel dieser Arbeit ist es, einen expliziten Zusammenhang zwischen $\operatorname{dem} C^{*}$-algebraischen Zugang und den Resultaten über topologische T-Dualität herzustellen. Dabei wird sich zeigen, daß wir dieses Ziel erreichen können, indem wir die lokale Struktur der zugrundeliegenden Objekte analysieren und mit Hilfe der gewonnenen lokalen Daten zeigen, daß in beiden Fällen die jeweiligen, geeignet gewählten Äquivalenzklassen der topologischen und $C^{*}$ algebraischen Objekte übereinstimmen.

Ich will mich an dieser Stelle recht herzlich bei all denjenigen bedanken, die zum Gelingen und Entstehen dieser Arbeit beigetragen haben. An erster Stelle gilt mein Dank natürlich meinem Doktorvater Herrn Ulrich Bunke, der sich (erstaunlicherweise) dazu bereit erklärte, mich als Doktorand zu betreuen, und mich immer wieder auf vielfältige Weise gefordert und gefördert hat. Ohne ihn wäre diese Arbeit tatsächlich nicht möglich gewesen, und da die mir aufgetragene Fragestellung im Grenzgebiet verschiedener, mathematischer Disziplinen liegt, konnte ich Dinge lernen, die mir unter anderen Umständen sicher verwehrt geblieben wären. Meinem Doktoronkel Herrn Thomas Schick und meinem Doktorbruder Herrn Moritz Wiethaup sei hier ausdrücklich für die zahlreichen, fruchtbaren Gespräche gedankt, ohne die ich einige Dinge nicht hätte so einfach oder schnell oder überhaupt hätte bewerkstelligen können. Dem Graduiertenkolleg Gruppen und Geometrie danke ich für das entgegengebrachte Vertrauen und die großzügige Unterstützung in den letzten zwei Jahren.

Nachdem mir von prominenter Stelle zugetragen wurde, daß es wohl an- 
gebracht ist, auch denjenigen zu danken, die indirekt an dieser Arbeit beteiligt sind, will ich manchen von meinen Lehrern und Hochschullehrern danken, von denen ich (natürlich in alphabetischer Folge) besonders die Herren Helmut Becker, Detlev Buchholz, Rüdiger Heidersdorf, Bernhard Meyer, KarlHenning Rehren und Wolfgang Watzlawek erwähnen will, die alle ihren besonderen Anteil an meinem Bildungsweg hatten und haben. Herrn Friedrich von Schiller danke ich für die Ode an die Freude, Herrn Ralf Lindemann für alles und Herrn Jens Latsch dafür, daß er mir im ersten Semester meine Oberonprogramme zugeschickt hat. Zum Schluß will ich meinen lieben Eltern und meiner lieben Großmutter dafür danken, daß diese Arbeit, nicht nur in der offensichtlichen Weise, ohne sie nicht hätte entstehen können.

Göttingen, im Oktober 2007 Ansgar Schneider 


\section{Contents}

1 Introduction and Summary $\quad 8$

2 Pairs and Triples 15

2.1 The Category of Pairs . . . . . . . . . . . . . . . . . 15

2.2 The Local Structure of Pairs and their Classifying Space . . . . . 18

2.3 Pairs and Twisted Čech Cohomology . . . . . . . . . . . . . . 20

2.4 Dynamical Triples and their Local Structure . . . . . . . . . . . . 23

2.5 Dual Pairs and Triples . . . . . . . . . . . . . . . . . . . . . 31

2.6 Topological Triples . . . . . . . . . . . . . . . . . 32

3 T-Duality 38

3.1 The Duality Theory of Dynamical Triples . . . . . . . . . . . 38

3.2 The Relation to Topological T-Duality . . . . . . . . . . . . . . . . . 47

3.3 The Case of $G=\mathbb{R}^{n}$ and $N=\mathbb{Z}^{n} \ldots \ldots \ldots$. . . . . . 71

3.4 The Structure of the Associated $C^{*}$-Dynamical Systems . . . . . 79

A Some Notation and Basic Lemmata $\quad \mathbf{8 8}$

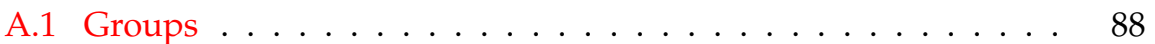

A.2 Group and Čech Cohomology . . . . . . . . . . . . . . . . 89

A.3 The Unitary and the Projective Unitary Group . . . . . . . . . . 89

A.4 Crossed Products . . . . . . . . . . . . . . . . . . . . . 92

A.5 Some Topology . . . . . . . . . . . . . . . 93

$\begin{array}{lr}\text { - Index } & 97\end{array}$

- References 99 


\section{Introduction and Summary}

String theory [Po] is based on the physical idea to describe nature not only by point particles but with the concept of higher dimensional objects. Although it is still unclear how close string theory is to physics a lot of interesting new phenomena have been observed which have led to many fruitfully new ideas and have made it a rich theory. In particular, a lot of new mathematical ideas have arose from the desire to understand the discovered structures. One of those is the concept of T-duality.

T-duality is a duality of string theories (type IIA and IIB) on different underlying space-time manifolds $E$ and ${ }^{1} \widehat{E}$ which are (in the simplest case) related by a transformation of type: radius $\mapsto 1$ /radius along a compactified space-time dimension. A duality between two theories on different space-time manifolds gives a prescription how the fields and their correlation functions transform under the change of the underlying manifolds. In the present case one may take as an example the charges of the D-branes which take values in the twisted K-theory of $E[\mathrm{BM}]$, where the twist is given by a background field on $E$ (a 3-form $H$ called $H$-flux). Then T-duality must give the answer how the background fields transform and should lead to an isomorphism of the twisted K-theories of the underlying manifolds.

We cannot give a summary of the whole subject, but we can try to point out some of its mathematical issues. In literature there are different approaches of a mathematical understanding of T-duality. One is based on the theory of $C^{*}$-dynamical systems which serve a notion of T-duality using crossed product $C^{*}$-algebras [BHM2, MR], another is by geometric and topological means [BEM, BHM1, BS, BRS], a third using methods from algebraic geometry [BSST]. We focus our attention to the first and second approach and continue to describe some features of the geometric-topological side in more detail.

Let us think of the manifolds $E$ and $\widehat{E}$ as principal circle bundles which have isomorphic quotients $E / S^{1} \cong \widehat{E} / S^{1}=: B$. In [BEM] it is described in terms of differential geometry how the data of the curvature $F$ of $E$ and of the $\mathrm{H}$-flux $H$ on $E$ are related to the corresponding dual data $\widehat{F}$ and $\widehat{H}$ of $\widehat{E}$. The result is that integration of $H$ along the fibres of $E$ yields the dual curvature and vice versa. In the case of $S^{1}$-bundles $E$ and $\widehat{E}$ we can identify the classes of the curvatures with the realifications of the first Chern classes $c_{1}, \hat{c}_{1} \in H^{2}(B, \mathbb{Z})$ of the respective bundles, and there also exist integer cohomology classes $h \in$ $H^{3}(E, \mathbb{Z}), \hat{h} \in H^{3}(\widehat{E}, \mathbb{Z})$ whose realifications are $h_{\mathbb{R}}=[H]$ and $\hat{h}_{\mathbb{R}}=[\widehat{H}]$. In this sense, we forgot geometry and now may only consider these topological data. This is the point of view which was adopted in [BS], wherein among other things the results of [BEM] are restated on a purely topological level. The higher dimensional case, where the circle $S^{1}$ is replaced by the $n$-dimensional torus $\mathbb{T}^{n}=\left(S^{1}\right)^{\times n}$, i.e. $E$ is thought of a principal $\mathbb{T}^{n}$-bundle, is described in [BHM1] in terms of differential geometry. Its topological structure is described

\footnotetext{
${ }^{1}$ The accent circonflexe ${ }^{\wedge}$ is going to be the most overloaded symbol in this work.
} 
in [BRS] which we want to discuss in more detail. They introduce so-called T-duality triples and define that a pair $(E, h)$ is dual to a pair $(\widehat{E}, \hat{h})$ if there is a T-duality triple connecting them. The notion of T-duality triples which we are going to call topological triples (Definition 2.9) is central for this work, so let us clarify what it means that a T-duality triple connects the pairs $(E, h)$ and $(\widehat{E}, \hat{h})$ :

A T-duality triple is a commutative diagram

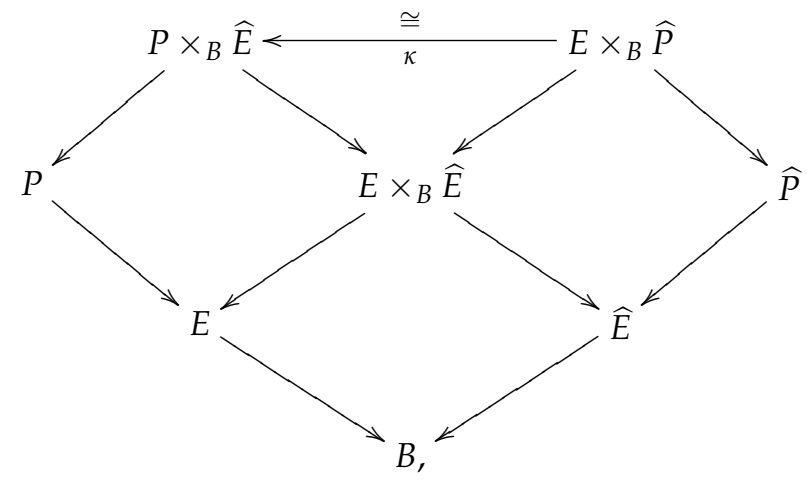

wherein $P \rightarrow E$ and $\widehat{P} \rightarrow \widehat{E}$ are principal bundles with structure group $\mathrm{PU}(\mathcal{H})$, the projective unitary group of some infinite dimensional, separable Hilbert space $\mathcal{H}$, such that both of these bundles are trivialisable when restricted to the fibres of $E \rightarrow B$ or $\widehat{E} \rightarrow B$ respectively. Moreover, the top-isomorphism $\kappa$ satisfies the following local condition: Due to the triviality condition on the bundles $P, \widehat{P}$, we can trivialise (1) over each $u \in B$ such that the isomorphism $\kappa$ induces a map $\kappa(u): \mathbb{T}^{n} \times \mathbb{T}^{n} \rightarrow \mathrm{PU}(\mathcal{H})$ which implements the isomorphism. Now, $\mathrm{PU}(\mathcal{H})$ is an Eilenberg-McLane space of type $K(\mathbb{Z}, 2)$ so $\kappa(u)$ defines a class $[\kappa(u)] \in H^{2}\left(\mathbb{T}^{n} \times \mathbb{T}^{n}, \mathbb{Z}\right)$. We force this class to satisfy $[\kappa(u)] \in \pi+$ $\operatorname{im}\left(\operatorname{pr}_{1}^{*}\right)+\operatorname{im}\left(\operatorname{pr}_{2}^{*}\right)$, where $\operatorname{pr}_{1,2}: \mathbb{T}^{n} \times \mathbb{T}^{n} \rightarrow \mathbb{T}^{n}$ are the projections and $\pi$ is the class of the tautological line bundle over $\mathbb{T}^{n} \times \mathbb{T}^{n}$ which is $\pi=y_{1} \cup \hat{y}_{1}+$ $\cdots+y_{n} \cup \hat{y}_{n}$, for the generators $y_{1}, \ldots, y_{n}, \hat{y}_{1}, \ldots, \hat{y}_{n}$ of $H^{1}\left(\mathbb{T}^{n} \times \mathbb{T}^{n}, \mathbb{Z}\right)$.

Remark 1.1 In fact, this is not the definition of [BRS]. Firstly, they uses the more general notion of twists instead of the bundles $P$ and $\widehat{P}$, but the category of $\mathrm{PU}(\mathcal{H})$ principal bundles with homotopy classes of bundle isomorphisms as morphisms is a model of twists. Secondly, they require the (a priori) more restrictive condition that the class of the bundles $[P] \in H^{3}(E, \mathbb{Z})$ (analogously for $[\widehat{P}] \in H^{3}(\widehat{E}, \mathbb{Z})$ ) lies in the second step $F^{2} H^{2}(E, \mathbb{Z})$ of the filtration $\{0\} \subset F^{3} H^{3}(E, \mathbb{Z}) \subset F^{2} H^{3}(E, \mathbb{Z}) \subset$ $F^{1} H^{3}(E, \mathbb{Z}) \subset H^{3}(E, \mathbb{Z})$ associated to the Leray-Serre spectral sequence. The requirement of triviality over the fibres of $E$ we stated above precisely means that the class lies in the first step $F^{1} H^{3}(E, \mathbb{Z})$ of the filtration. However, these two conditions on the classes are equivalent as we show in Lemma 3.7.

A T-duality triple which we denote by $(\kappa,(P, E),(\widehat{P}, \widehat{E}))$ connects the two pairs $(E, h)$ and $(\widehat{E}, \hat{h})$ if we have an equality of the classes $[P]=h$ and $[\widehat{P}]=\hat{h}$. 
To a T-duality triple we can associate two $C^{*}$-algebras, namely the $C^{*}$-algebra of sections $\Gamma(E, F)$ and $\Gamma(\widehat{E}, \widehat{F})$ of the associated bundles $F:=P \times_{\mathrm{PU}(\mathcal{H})} \mathcal{K}(\mathcal{H})$ and $\widehat{F}:=\widehat{P} \times{ }_{\mathrm{PU}(\mathcal{H})} \mathcal{K}(\mathcal{H})$. It is the very aim of this work to understand how these two $C^{*}$-algebras are related to each other. This issue turns our focus on the $C^{*}$-algebraic approach to T-duality [MR, BHM2] which is based on the understanding of abelian $C^{*}$-dynamical systems.

We shortly summarise some $C^{*}$-algebraic background.

The duality theory of abelian $C^{*}$-dynamical systems which has been investigated for a quite long time [Pe1] is the foundation to understand T-duality by $C^{*}$-algebraic means. The dual of an abelian $C^{*}$ dynamical system $(A, G, \alpha)$, i.e. $A$ a $C^{*}$-algebra with strongly continuous action $\alpha: G \rightarrow \operatorname{Aut}(A)$ of a locally compact, abelian group $G$, is the crossed product $C^{*}$-algebra $G \times_{\alpha} A$ equipped with the natural action $\hat{\alpha}$ of the dual group $\widehat{G}$, i.e. $\left(G \times_{\alpha} A, \widehat{G}, \hat{\alpha}\right)$ becomes again a $C^{*}$-dynamical system (see [Pe1] or section A.4). A central result is the Takai duality theorem (Theorem A.2) which states in particular that the bi-dual $C^{*}$ algebra is stably isomorphic to the original one, i.e. they are Morita equivalent. Thus, it is completely trivial to understand the structure of the bi-dual and the difficult task is to understand the dual $G \times{ }_{\alpha} A$.

In the $80 \mathrm{~s}$ and $90 \mathrm{~s}$ big progress has been made to understand the dual in case $A$ is a continuous trace algebra which we assume from now on. The basic structure theorem of Dixmier and Douady (see e.g. [Di]) says that any separable, stable continuous trace algebra $A$ is isomorphic to $\Gamma_{0}(E, F)$ the $C^{*}$-algebra of sections vanishing at infinity, where $E:=\operatorname{spec}(A)$ is the spectrum of $A$ and $F \rightarrow E$ is a locally trivial bundle with each fibre isomorphic to the compacts $\mathcal{K}(\mathcal{H})$. Their isomorphism classes are classified by $\check{H}^{2}(E, \mathrm{U}(1)) \cong H^{3}(E, \mathbb{Z})$ (cp. section A.3), and the class in $H^{3}(E, \mathbb{Z})$ which determines the isomorphism type of $A \cong \Gamma_{0}(E, F)$ is called the Dixmier-Douady invariant of $A$.

A first result [Pe2, RW] for an understanding the crossed product $G \times{ }_{\alpha} A$ was that if $G$ is compactly generated and the induced action of $G$ on the spectrum $E$ of $A$ is trivial, then the crossed product $G \times_{\alpha} A$ is isomorphic to the balanced tensor product $C_{0}(\widehat{E}) \otimes_{\mathcal{C}_{0}(B)} A=: p^{*} A$, wherein $p: \widehat{E} \rightarrow B$ is a $\widehat{G}$ principal bundle consisting of the spaces $\widehat{E}:=\operatorname{spec}\left(G \times_{\alpha} A\right)$ and $B:=E$.

The more general situation wherein the action of $G$ does not fix the spectrum $E$ of $A$ but has constant isotropy group $N$ for each $\pi \in E$ is concerned in [RR]. One of the statements therein is the following. Assume that $E$ with the induced action of $G / N$ is a principal fibre bundle $E \rightarrow B:=E /(G / N)$ and that the restricted action $\left.\alpha\right|_{N}$ of $N$ on $A$ is locally unitary, then there is a pull 
back diagram of principal fibre bundles

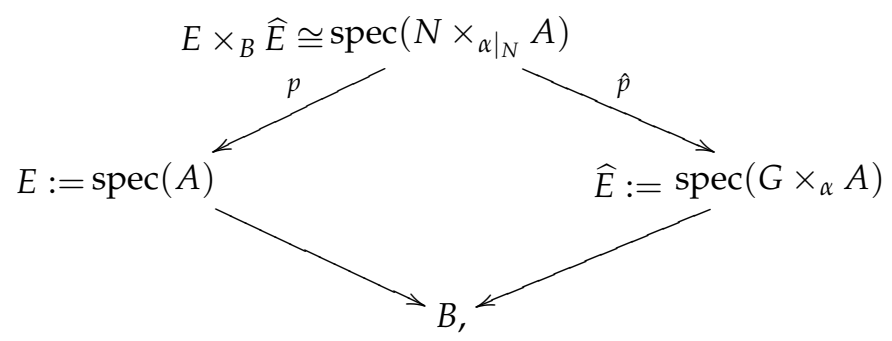

wherein the down-right arrows have fibre $G / N$ and the down-left arrows have fibre $\widehat{N} \cong \widehat{G} / N^{\perp}$. ( $N^{\perp}$ is the annihilator of $N$ which is the set of characters of $G$ whose restriction to $N$ is identically 1.) Moreover, $p^{*} A$ is isomorphic to $N \times{ }_{\left.\alpha\right|_{N}} A$ and Morita equivalent to $\hat{p}^{*}\left(G \times_{\alpha} A\right)$. Thus, we have the following schematic situation of $C^{*}$-algebras over their spectra

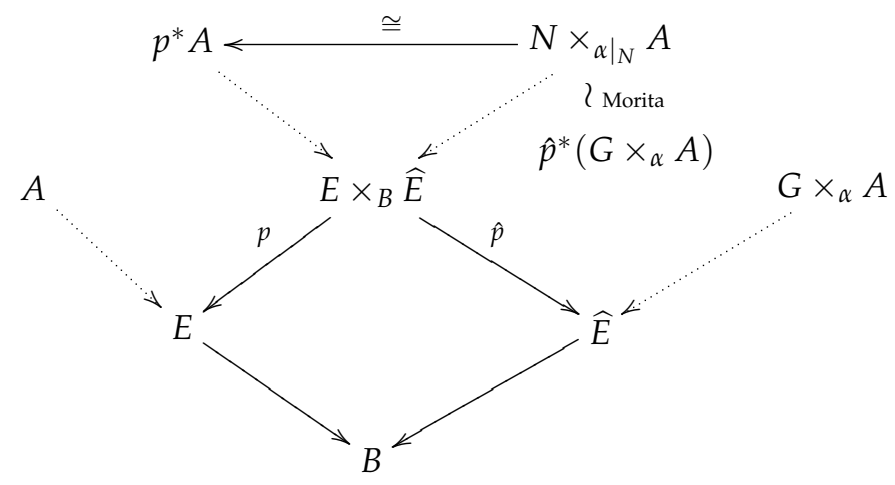

which obviously is similar to diagram (1). The question is whether or not it is possible that both $A$ and $G \times_{\alpha} A$ are separable, stable continuous trace algebras. This question has been answered in [ER, Thm. 6]. In particular, this is true if $\left.\alpha\right|_{N}$ is point-wise unitary and the action of $G / N$ on $E \times{ }_{B} E \cong \operatorname{spec}\left(N \times{ }_{\left.\alpha\right|_{N}} A\right)$ is proper, e.g. $G / N$ is compact.

This finishes our summary.

The approach to T-duality of [MR] considers the following set-up. Let $E$ be a locally compact space (with certain finiteness assumptions) with an action of the torus $\mathbb{T}^{n}$ such that $E \rightarrow B:=E / \mathbb{T}^{n}$ becomes a principal torus bundle, and let $h \in H^{3}(E, \mathbb{Z})$. They concern these data as a stable continuous trace algebra $\Gamma_{0}(E, F)$ that has Dixmier-Douady invariant $h$. Under which circumstances is it possible to lift the $\mathbb{T}^{n}$-action from the spectrum $E$ to an action of $\mathbb{R}^{n}=: G$ on $\Gamma_{0}(E, F)$ ? If so, is it further possible to obtain an action whose restriction to $N:=\mathbb{Z}^{n}$ is point-wise unitary for all $\pi \in E$ ? These questions are answered in [MR, Thm 3.1]. The general answer is no, but if we make further restrictions 
to the class $h$ one achieves a positive answer. Namely, a lift $\alpha$ to an $\mathbb{R}^{n}$-action exists if and only if $h \in F^{1} H^{3}(E, \mathbb{Z})$ the first step of the filtration

$$
0 \subset F^{3} H^{3}(E, \mathbb{Z}) \subset F^{2} H^{3}(E, \mathbb{Z}) \subset F^{1} H^{3}(E, \mathbb{Z}) \subset H^{3}(E, \mathbb{Z})
$$

associated to the Leray-Serre spectral sequence, and there exists a point-wise unitary action if even $h \in F^{2} H^{3}(E, \mathbb{Z})$. Consequently, in the latter case there exists a T-dual in the sense that there exists a dual space-time $\widehat{E}$ in diagram (2) which is a $\widehat{\mathbb{Z}^{n}} \cong \mathbb{T}^{n}$-principal fibre bundle over $B$ and is the spectrum of the stable continuous trace algebra $\mathbb{R}^{n} \times_{\alpha} \Gamma(E, F) \cong \Gamma_{0}(\widehat{E}, \widehat{F})$.

In this work we are going to show that the two approaches to T-duality are essentially equivalent, i.e. there is no difference between (equivalence classes of) T-duality triples and (equivalence classes of) abelian $C^{*}$-dynamical systems which are obtained as described above. To fulfil this task we must develop a technique which enables us to compare such different objects. The methods of [BRS] and [MR] are not applicable for such a manoeuvre as they are too less explicit: The explicit description of the local structure of these two different kinds of objects can be used to describe a transformation as desired.

Our method is general enough that we do not have to restrict ourselves to the case of the groups $\mathbb{R}^{n}$ and $\mathbb{T}^{n} \cong \mathbb{R}^{n} / \mathbb{Z}^{n}$. - We develop a theory for all second countable, locally compact, abelian groups $G$ with lattice $N \subset G$, i.e. $N$ is a discrete, cocompact subgroup.

We give an overview of this work.

A basic technique we use throughout the whole of this work is to lift all local, projective unitary families of functions (e.g. the transition functions of a PU(F)-principal bundle $P \rightarrow E$ ) to unitary Borel- or $L^{\infty}$-functions in direction of the fibres $G / N$ of $E \rightarrow B$ and to think of them as new unitary (multiplication) operators in $\mathrm{U}\left(L^{2}(G / N) \otimes \mathcal{H}\right)$. We call this procedure Borel lifting technique. The technical condition we must assume is that the base $B$ is a paracompact Hausdorff space which is locally contractible. We call spaces with these properties base spaces, and the whole theory we develop is a theory over base spaces.

In sections 2.1 and 2.2 we introduce the notion of pairs. A pair is a $G / N-$ principal fibre bundle $E \rightarrow B$ over a base space $B$ and a $\mathrm{PU}(\mathcal{H})$-principal fibre bundle $P \rightarrow E$ which is trivialisable over the fibres of $E$. We explain their local structure and give a quick result on their classification.

In section 2.3 we introduce a twisted version of Čech cohomology on the base $B$, where the twist is given by the bundle $E \rightarrow B$. The Borel lifting technique mentioned above defines a map from (equivalence classes of) pairs into the second twisted Čech cohomology (similar to the ordinary definition of the second Čech class of a PU(H)-bundle).

In sections 2.4 we extend the same procedure to dynamical triples $(\rho, E, P)$ which are pairs $(P, E)$ equipped with a lift $\rho$ of the $G / N$ action on $E$ to a $G$ action on $P$. Due to this action, group cohomological expressions arise in the 
description of their local structure and lead finally to a map from (equivalence classes of) dynamical triples to the second cohomology of a double complex which has one (twisted) Cech cohomological direction and a second group cohomological direction. A two cocycle (or two cohomology class) of this complex has three entries: a pure Čech part due to the transition functions of the pair, a group cohomological part and a third mixed term. In section 3.1 we focus our attention to those triples for which the group cohomological entry vanishes.

Section 2.5 just contains the definition of dual pairs and dual dynamical triples which are nothing more than pairs and dynamical triples, but as underlying groups we take the dual group $\widehat{G}$ of $G$ with dual lattice $N^{\perp}$ the annihilator of $N$.

Then in section 2.6 we introduce topological triples. Our definition is a straight forward generalisation to arbitrary locally compact, abelian groups $G$ with lattice $N$ from the notion of a T-duality triple $\left(G=\mathbb{R}^{n}, N=\mathbb{Z}^{n}\right)$.

In section 3.1 we state our first main result. We first single out a specific subclass of dynamical triples which we call dualisable. They are those dynamical triples for which the group cohomological entry of the associated two cohomology class (of the double complex) vanishes. We construct an explicit map $[(\rho, E, P)] \mapsto[(\hat{\rho}, \widehat{E}, \widehat{P})]$ from the set of equivalence classes of dualisable dynamical triples to the set of equivalence classes of dualisable dual dynamical triples, and show that it is a bijection whose inverse is given by the dual map (defined by replacing everything by its dual counterpart). In this sense dualisable dynamical triples and dual dualisable triples are in duality.

Section 3.2 contains two important statements. The first is that we have a map $\tau(B)$ from the set of equivalence classes of dualisable dynamical triples to the set of equivalence classes of topological triples (everything understood over a base space $B$ ). This map is defined by the duality theorem of section 3.1 , i.e. the topological triple we define consists of the pairs of two dynamical triples in duality. Then we try to define a map $\delta(B)$ in the opposite direction which generally fails as an obstruction occurs. However, on the subset of those topological triples which have a vanishing obstruction we then find a construction of a whole family of dualisable dynamical triples which is associated to a topological triple. This construction, when restricted to the image of the first map, can be turned into an honest map, i.e. we have a preferred choice of an element of the family, and this map is inverse to $\tau(B)$.

Section 3.3 is devoted to the special case of the group $G=\mathbb{R}^{n}$ with lattice $N=\mathbb{Z}^{n}$. In this situation the construction of the map $\delta(B)$ simplifies drastically, the group wherein the obstruction lives vanishes. As a result, we can associate to each topological triple a dynamical triple which is unique (up to equivalence) because the family of dynamical triples degenerates to a family of one single element only. As it turns out the two maps $\tau(B)$ and $\delta(B)$ are bijections and inverse to each other.

Moreover, the four maps mentioned above are natural in the base, so they define natural transformations of functors. Thus, the main result of section 3.3 
can be restated as follows. In the case of $G=\mathbb{R}^{n}$ and $N=\mathbb{Z}^{n}$, we have a completely explicit construction of equivalences of functors

$$
\mathrm{Top} \cong \mathrm{Dyn}^{+} \cong \widehat{\mathrm{Dyn}}^{+}
$$

Therein, $\mathrm{Dyn}^{\dagger}\left(\widehat{\mathrm{Dyn}}^{+}\right)$is the functor sending a base space to the set of equivalence classes of dualisable (dual) dynamical triples over it. Top is the functor which sends a base space to the set of equivalence classes of topological ( $\mathrm{T}$ duality) triples over it.

In section 3.4 we point out that the theory developed so far is connected to the theory of $C^{*}$-dynamical systems precisely as one expects. Namely, the $C^{*}$ dynamical systems $\left(G \times \alpha_{\alpha \rho} \Gamma(E, F), \widehat{G}, \widehat{\alpha \rho}\right)$ and $\left(\Gamma(\widehat{E}, \widehat{F}), \widehat{G}, \alpha^{\hat{\rho}}\right)$ are isomorphic, wherein $(\hat{\rho}, \widehat{E}, \widehat{P})$ is the dual of $(\rho, E, P)$ and $(\rho, E, P) \mapsto\left(\Gamma(E, F), G, \alpha^{\rho}\right)$ is the functor which sends a dualisable (dual) dynamical triple to its corresponding $C^{*}$-dynamical system, i.e. $F$ is the associated $\mathcal{K}(\mathcal{H})$-bundle to $P$ and $\alpha^{\rho}$ is the by $\rho$ induced action on the $C^{*}$-algebra of sections $\Gamma(E, F)$.

In an appendix we put some technical lemmata and notation we are going to use. 


\section{Pairs and Triples}

\subsection{The Category of Pairs}

Definition 2.1 A base space B is a topological space which is Hausdorff, paracompact and locally contractible.

The category of base spaces consists of bases spaces as objects and continuous maps between them as morphisms. A typical class of base spaces are CW-complexes [FP, Thm. 1.3.2, Thm. 1.3.5].

By $G$ we will always denote a second countable, Hausdorff, locally compact abelian group and by $N$ a discrete, cocompact subgroup, i.e. the quotient $G / N$ is compact.

Let $\mathcal{H}$ be an infinite dimensional, separable Hilbert space. Let $E \rightarrow B$ be a $G / N$-principal fibre bundle and $P \rightarrow E$ be a $\mathrm{PU}(\mathcal{H})$-principal fibre bundle.

Definition 2.2 We call the data ${ }^{2}(P, E)$ a pair over B with underlying Hilbert space $\mathcal{H}$ if

i) B is a base space,

ii) the restriction of the bundle $P \rightarrow E$ to the fibres of $E \rightarrow B$ is trivialisable.

Remark 2.1 We do not require local compactness for $B$, because we want to develop a theory that includes non locally compact spaces such as classifying spaces of groups (s. next section). Therefore $E$ need not to be locally compact and thus need not equal the spectrum of any (continuos trace) $C^{*}$-algebra such as $\Gamma(E, F)$ the $C^{*}$-algebra of bounded sections (or $\Gamma_{0}(E, F)$ the $C^{*}$-algebra of sections vanishing at infinity [A section vanishing at infinity vanishes already identically on the set of points which don't have a compact neighbourhood.]) of the associated $C^{*}$-bundle $F:=P \times_{\mathrm{PU}(\mathcal{H})} \mathcal{K}(\mathcal{H})$.

A morphism $(\varphi, \vartheta, \theta)$ over $B$ from a pair $P \rightarrow E \rightarrow B$ with underlying Hilbert space $\mathcal{H}$ to a pair $P^{\prime} \rightarrow E^{\prime} \rightarrow B$ with underlying Hilbert space $\mathcal{H}^{\prime}$ is a commutative diagram of bundle isomorphisms

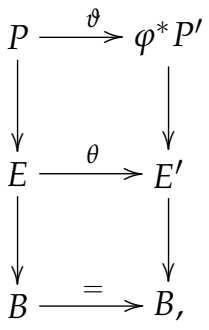

wherein $\varphi \in \operatorname{PU}\left(\mathcal{H}, \mathcal{H}^{\prime}\right):=\mathrm{U}\left(\mathcal{H}, \mathcal{H}^{\prime}\right) / \mathrm{U}(1)$ is the class of a unitary isomorphism $\mathcal{H} \rightarrow \mathcal{H}^{\prime} . \varphi^{*} P^{\prime}$ is the $\mathrm{PU}(\mathcal{H})$-bundle with total space $\varphi^{*} P^{\prime}=P^{\prime}$, but with $\mathrm{PU}(\mathcal{H})$-action that is induced by $\varphi^{*}: \mathrm{PU}(\mathcal{H}) \rightarrow \mathrm{PU}\left(\mathcal{H}^{\prime}\right)$, i.e. $x^{\prime} \cdot U:=$

${ }^{2}$ differing from [BRS] 
$x^{\prime} \cdot\left(\varphi^{*}\right)^{-1} U, x^{\prime} \in P^{\prime}, U \in \mathrm{PU}(\mathcal{H})$. Pairs over a base space $B$ and their morphisms form a category; composition of morphisms $(\varphi, \vartheta, \theta)$ and $\left(\varphi^{\prime}, \vartheta^{\prime}, \theta^{\prime}\right)$ is just component-wise composition $\left(\varphi^{\prime} \circ \varphi, \vartheta^{\prime} \circ \vartheta, \theta^{\prime} \circ \theta\right)$. This category is even a groupoid, i.e. every morphism is an isomorphism.

This notion of morphism is well-behaved under stabilisation in the following sense. Let $(P, E)$ be a pair over $B$ with underlying Hilbert space $\mathcal{H}$. Let $\mathcal{H}_{0}$ be any separable Hilbert space, not neccessarily infinite dimensional. Then $\mathrm{PU}(\mathcal{H})$ is isomorphic to the subgroup $\mathbb{1} \otimes \mathrm{PU}(\mathcal{H})$ of $\mathrm{PU}\left(\mathcal{H}_{0} \otimes \mathcal{H}\right)$ and thus $\mathrm{PU}(\mathcal{H})$ acts on $\mathrm{PU}\left(\mathcal{H}_{0} \otimes, \mathcal{H}\right)$ by left multiplication: $(U, V) \mapsto\left(\mathbb{1}_{\mathcal{H}_{0}} \otimes U\right) V$. Then the associated (stabilised) bundle

$$
P_{\mathcal{H}_{0}}:=\mathrm{PU}\left(\mathcal{H}_{0}\right) \otimes P:=P \times_{\mathrm{PU}(\mathcal{H})} \mathrm{PU}\left(\mathcal{H}_{0} \otimes \mathcal{H}\right)
$$

is a $\mathrm{PU}\left(\mathcal{H}_{0} \otimes \mathcal{H}\right)$-principal bundle and $\left(P_{\mathcal{H}_{0}}, E\right)$ is a pair over $B$ with underlying Hilbert space $\mathcal{H}_{0} \otimes \mathcal{H}$. We call two pairs $(P, E)$ and $\left(P^{\prime}, E^{\prime}\right)$ with underlying Hilbert spaces $\mathcal{H}$ and $\mathcal{H}^{\prime}$ stably isomorphic if there exists a Hilbert space $\mathcal{H}_{0}$ such that the pairs $\left(P_{\mathcal{H}_{0}}, E\right)$ and $\left(P_{\mathcal{H}_{0}}^{\prime}, E^{\prime}\right)$ are isomorphic.

Proposition 2.1 Two pairs over B are stably isomorphic if and only if they are isomorphic.

Proof : It is clear that isomorphic pairs are stably isomorphic. To prove the converse it suffices to show that $P$ and $\varphi^{*} P_{\mathcal{F}_{0}}$ are isomorphic over $E$, for an isomorphism $\varphi: \mathcal{H} \cong \mathcal{H}_{0} \otimes \mathcal{H}$. To do so, we show that $P$ and $\varphi^{*} P_{\mathcal{H}_{0}}$ define the same Čech class, hence the classification theorem of $\mathrm{PU}(\mathcal{H})$-bundles (Theorem A.1) implies that the two bundles are isomorphic.

In fact, if $\zeta_{j i}^{1}: V_{j i} \rightarrow \mathrm{PU}(\mathcal{H})$ are transition functions for $P$ (here $\left\{V_{i}\right\}$ is a covering of $E)$, then $\zeta_{j i}^{2}:=\varphi^{*}\left(\mathbb{1} \otimes \zeta_{j i}\right)$ are transition functions for $\varphi^{*} P_{\mathcal{F}_{0}}$. If we refine the covering such that the transition functions $\zeta_{j i}^{1}$ lift to unitaryvalued functions $\bar{\zeta}_{j i}^{1}$ (Lemma A.8), then these lifts define also lifts $\bar{\zeta}_{j i}^{2}$ for the other family of transition functions. Thus, on threefold intersections $V_{k j i}$ the cocycle identities of the two families are perturbed by the same Čech 2-cocycle

$$
c_{k j i}:=\bar{\zeta}_{j i}^{n} \bar{\zeta}_{k i}^{n-1} \bar{\zeta}_{k j}^{n}: V_{k j i} \rightarrow \mathrm{U}(1) \cdot \mathbb{1}, \quad n=1,2 .
$$

Hence, their classes agree.

Remark 2.2 In case of an additional structure such as a group action of $G$ on $P$ stable isomorphism and isomorphism are different notions when we force them to preserve the extra structure. This will be important in section 2.4.

Let us denote by Par the set valued contravariant functor that sends a base space $B$ to the set of stable isomorphism classes of pairs over $B$, i.e.

$$
\operatorname{Par}(B):=\{\text { pairs over } B\} / \text { stable isomorphism },
$$


and if $f: B^{\prime} \rightarrow B$ is a continuous map between base spaces, then pullback defines a map $f^{*}: \operatorname{Par}(B) \rightarrow \operatorname{Par}\left(B^{\prime}\right)$.

There is a subcategory of pairs over $B$ consisting of pairs with a fixed $G / N$ bundle $E \rightarrow B$ and morphisms of the form $\left(\varphi, \vartheta, \mathrm{id}_{E}\right)$, and we call pairs $(P, E)$ and $\left(P^{\prime}, E\right)$ stably isomorphic over $E$ if there is a isomorphism of this special form between $\left(P_{\mathcal{H}_{1}}, E\right)$ and $\left(P_{\mathcal{H}_{1}}^{\prime}, E\right)$, for a Hilbert space $\mathcal{H}_{1}$. We define

$$
\operatorname{Par}(E, B):=\{\text { pairs over } B \text { with fixed } E\} / \text { stable isomorphism over } E^{\prime}
$$

and for a bundle morphism

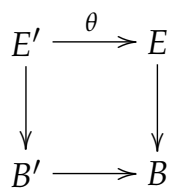

we define by pullback a map $\theta^{*}: \operatorname{Par}(E, B) \rightarrow \operatorname{Par}\left(E^{\prime}, B^{\prime}\right)$, so $\operatorname{Par}(., .$.$) be-$ comes a contravariant functor from the category of $G / N$-principal fibre bundles over base spaces to sets. The bundle automorphisms $\operatorname{Aut}_{B}(E)$ of $E$ over $\operatorname{id}_{B}$ act on $\operatorname{Par}(E, B)$ by pullback, and we have a decomposition $\operatorname{Par}(B) \cong$ $\amalg_{[E]}\left(\operatorname{Par}(E, B) / \operatorname{Aut}_{B}(E)\right)$, wherein the disjoint union runs over all isomorphism classes of $G / N$-bundles $E \rightarrow B$.

Remark 2.3 For each fixed $E \rightarrow B$ the set $\operatorname{Par}(E, B)$ has a natural group structure. If $[(P, E)]$ and $\left[\left(P^{\prime}, E\right)\right]$ are two classes of pairs, then we let $[(P, E)]+\left[\left(P^{\prime}, E\right)\right]:=$ $\left[\left(P \otimes P^{\prime}, E\right)\right]$, wherein $P \otimes P^{\prime}$ is the $\mathrm{PU}\left(\mathcal{H} \otimes \mathcal{H}^{\prime}\right)$-bundle which is associated to the $\mathrm{PU}(\mathcal{H}) \times \mathrm{PU}\left(\mathcal{H}^{\prime}\right)$-bundle $P \times{ }_{E} P^{\prime}$,

$$
P \otimes P^{\prime}:=\left(P \times{ }_{E} P^{\prime}\right) \times \mathrm{PU}(\mathcal{H}) \times \mathrm{PU}\left(\mathcal{H}^{\prime}\right) \mathrm{PU}\left(\mathcal{H} \otimes \mathcal{H}^{\prime}\right) .
$$

The unit element is given by the class of a trivial bundle and the inverse of $[(P, E)]$ is given by the class $\left[\left(P^{\#}, E\right)\right]$ of the complex conjugate bundle $P^{\#}$ which is as space the bundle $P$ but has the action $(x, U) \mapsto x \cdot U^{\#}$. $U^{\#}$ is here the complex conjugate (not the adjoint) of $U \in \mathrm{PU}(\mathcal{H})$ (which may be defined by identifying $\mathcal{H}=l^{2}(\mathbb{N})$ and taking the complex conjugate matrix of $u=\left(u_{i j}\right)_{i, j \in \mathbb{N}}$, for $\left.U=\operatorname{Ad}(u)\right)$.

In this way we just mimic the group structure of $\breve{H}^{2}(E, \mathrm{U}(1))$, i.e. the classification map $\operatorname{Par}^{3}(E, B) \rightarrow \breve{H}^{2}(E, \underline{\mathrm{U}(1)})$ is turned into a group homomorphism.

An automorphism of a pair is a morphism from a pair onto itself. The group of automorphisms of a pair is denoted by $\operatorname{Aut}(P, E)$. It becomes a topological group when equipped with the initial topology of the forgetful map $\operatorname{Aut}(P, E) \rightarrow \operatorname{PU}\left(\mathcal{H}, \mathcal{H}^{\prime}\right) \times \operatorname{Map}(P, P)$ which sends a morphism $(\varphi, \vartheta, \theta)$ to $(\varphi, \vartheta)$, wherein $U\left(\mathcal{H}, \mathcal{H}^{\prime}\right)$ has the strong topology, i.e. the topology of pointwise convergence. $\operatorname{Map}(P, P)$ has the compact open topology.

Since $G / N$ is a commutative group mappings of the form $\theta_{z}: E \ni e \mapsto$ $e \cdot z \in E, z \in G / N$, are bundle morphisms. They give rise to a subgroup

\footnotetext{
${ }^{3}$ See Theorem A.1.
} 
$\operatorname{Aut}_{1}(P, E)$ which consist of all morphisms $\left(\operatorname{id}_{\mathcal{H}}, \vartheta, \theta_{z}\right)$. Let $\operatorname{Aut}_{0}(P, E)$ denote the subgroup consisting of morphisms $\left(\operatorname{id}_{\mathcal{H}}, \vartheta, \mathrm{id}_{E}\right)$, then we find a short exact sequence of topological groups

$$
\mathbb{1} \rightarrow \operatorname{Aut}_{0}(P, E) \rightarrow \operatorname{Aut}_{1}(P, E) \rightarrow G / N \rightarrow 0 .
$$

\subsection{The Local Structure of Pairs and their Classifying Space}

By $\mathcal{A}$ we denote the group of automorphisms of the trivial pair over a point. In particular, $a=(\varphi, \vartheta, \theta) \in \mathcal{A}$ makes

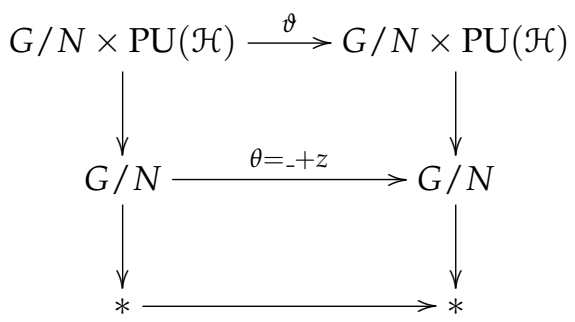

commute, for some $z \in G / N$. It is immediate that there is an isomorphism

$$
\mathcal{A} \cong G / N \ltimes \operatorname{Map}(G / N, \operatorname{PU}(\mathcal{H})) \rtimes \operatorname{PU}(\mathcal{H})
$$

of topological groups, wherein $G / N \ltimes \operatorname{Map}(G / N, \mathrm{PU}(\mathcal{H})) \rtimes \mathrm{PU}(\mathcal{H})$ is the semidirect product with multiplication $(y, \eta, u) \cdot(z, \zeta, v):=(y+z,(z \cdot \eta \cdot v) \zeta, u v)$. The action on the continuous functions $\operatorname{Map}(G / N, \mathrm{PU}(\mathcal{H}))$ is

$$
(z \cdot \eta \cdot v)(x):=v^{-1} \eta(x+z) v .
$$

Let $(P, E)$ be any pair over $B$ with underlying Hilbert space $\mathcal{H}$. $B$ is a base space, and so we can choose a covering $\left\{U_{i} \mid i \in I\right\}$ of $B$ of open sets such that for each $U_{i}$ there is a commutative diagram

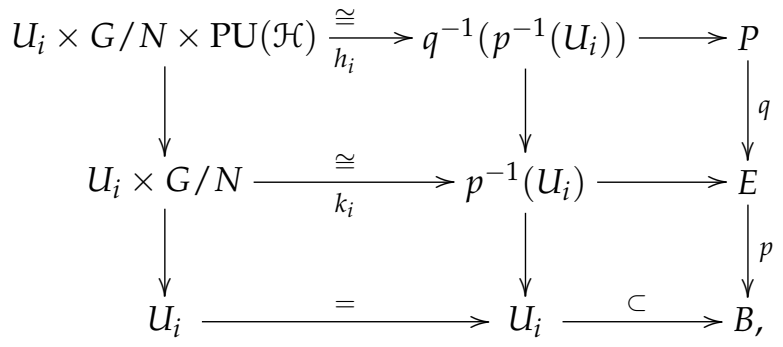

with bundle isomorphisms $k_{i}, h_{i}$. We refer to such a covering as an atlas $U_{\bullet}=$ $\left\{\left(U_{i}, k_{i}, h_{i}\right) \mid i \in I\right\}$ consisting of the charts $\left(U_{i}, k_{i}, h_{i}\right)$. The transition from one chart to another is described by a set of transition functions. For a pair 
this consists of two families of continuous functions $g_{i j}: U_{i j} \rightarrow G / N$ and $\zeta_{i j}: U_{i j} \rightarrow \operatorname{Map}(G / N, \operatorname{PU}(\mathcal{H}))$ which appear in

$$
\begin{aligned}
h_{j}^{-1} \circ h_{i}: U_{j i} \times G / N \times \mathrm{PU}(\mathcal{H}) & \rightarrow U_{i j} \times G / N \times \mathrm{PU}(\mathcal{H}) . \\
(u, z, U) & \mapsto\left(u, g_{j i}(u)+z, \zeta_{j i}(u)(z) U\right)
\end{aligned}
$$

It follows that on threefold intersections $U_{i j k}$ the relations $g_{k i}(u)=g_{k j}(u)+$ $g_{j i}(u)$ and

$$
\zeta_{k i}(u)(z)=\zeta_{k j}(u)\left(g_{j i}(u)+z\right) \zeta_{j i}(u)(z) \in \mathrm{PU}(\mathcal{H})
$$

are valid; equivalently, the family of functions

$$
a_{i j}:=g_{i j} \times \zeta_{i j}: U_{i j} \rightarrow G / N \ltimes \operatorname{Map}(G / N, \operatorname{PU}(\mathcal{H}))=: \mathcal{A}_{1} \subset \mathcal{A}
$$

satisfies the Čech 1-cocycle condition $a_{i j}(u) a_{j k}(u)=a_{i k}(u)$. Now, let $\mathcal{E} \mathcal{A}_{1} \rightarrow$ $\mathcal{B} \mathcal{A}_{1}$ be the universal $\mathcal{A}_{1}$-principal fibre bundle. We call the associated pair

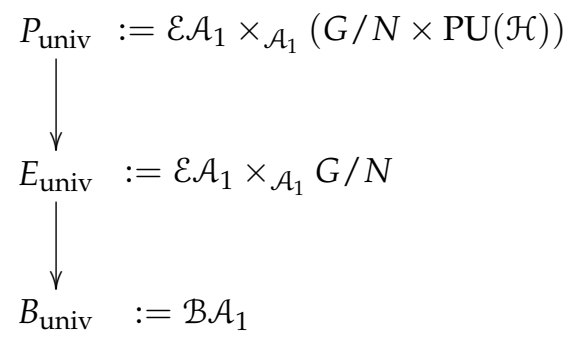

the universal pair. Indeed we can choose a $\mathrm{CW}$-model for $\mathcal{B} \mathcal{A}_{1}$ such that the universal pair is a pair in the sense of Definition 2.2. Its name is due to the following universal property.

Proposition 2.2 The space $B_{\text {univ }}$ classifies pairs over (pointed) $C W$-complexes, i.e. if $B$ is a (pointed) $C W$-complex, then

$$
\left[B, B_{\text {univ }}\right] \cong \operatorname{Par}(B),
$$

wherein the left hand side is the set of (pointed) homotopy classes of maps $B \rightarrow B_{\text {univ }}$.

Proof : We already observed that the transition functions of a pair define a Čech class $\left[a_{\text {... }}\right]=\left[g_{\text {.. }} \times \zeta_{\text {.. }}\right] \in \breve{H}^{1}\left(B, \mathcal{A}_{1}\right)$. This class is independent of the chosen atlas. Now, let $(\varphi, \vartheta, \theta):(P, E) \rightarrow\left(\overline{P^{\prime}}, E^{\prime}\right)$ be an isomorphism of pairs, then the transition functions $a^{\prime}$. for $\left(P^{\prime}, E^{\prime}\right)$ can be turned into a Čech $\mathcal{A}_{1}$-cocycle induced by $\varphi^{*}: \operatorname{PU}(\mathcal{H}) \cong \operatorname{PU}\left(\mathcal{H}^{\prime}\right)$, and this class depends on the isomorphism class of the pair only. Conversely, the associated pairs of isomorphic $\mathcal{A}_{1}$-principal bundles are isomorphic, and each isomorphism class arises.

Thus, pairs and $\mathcal{A}_{1}$-principal bundles over $B$ have the same isomorphism classes, but for a (pointed) $\mathrm{CW}$-complex $B$ the latter isomorphism class is given by homotopy classes of maps to $\mathcal{B} \mathcal{A}_{1}$. 


\subsection{Pairs and Twisted Čech Cohomology}

Let $\mathcal{M}$ and $\mathcal{G}$ be abelian (pre-)sheaves on a space $B$ and assume that $\mathcal{M}$ is a right $\mathcal{G}$ module. We are going to twist the Čech coboundary operator of $\mathcal{M}$ by a Čech $\mathcal{G}$ 1-cocycle. Fix an open covering $U_{\bullet}=\left\{U_{i} \mid i \in I\right\}$ of $B$ and let $g \in \check{Z}^{1}\left(U_{\bullet}, \mathcal{G}\right)$. Then for $\varphi \in \check{C}^{n-1}\left(U_{\bullet}, \mathcal{N}\right), n=1,2, \ldots$, we define

$$
\delta_{g} \varphi:=\delta \varphi+g^{\star} \varphi,
$$

wherein $\delta$ is the ordinary Čech coboundary operator ${ }^{4}$ and

$$
\left(g^{\star} \varphi\right)_{i_{0} \ldots i_{n}}:=\left.(-1)^{n-1}\left(\varphi_{i_{0} \ldots i_{n-1}}-\varphi_{i_{0} \ldots i_{n-1}} \cdot g_{i_{n-1} i_{n}}\right)\right|_{U_{i_{0} \ldots i_{n}}} .
$$

The choice of the sign $(-1)^{n-1}$ is such that the last term of $\delta \varphi$ and the first term of $g^{\star} \varphi$ cancel. We obtain a sequence

$$
0 \longrightarrow \check{C}^{0}\left(U_{\bullet}, \mathcal{M}\right) \stackrel{\delta_{g}}{\longrightarrow} \check{C}^{1}\left(U_{\bullet}, \mathcal{M}\right) \stackrel{\delta_{g}}{\longrightarrow} \check{C}^{2}\left(U_{\bullet}, \mathcal{M}\right) \stackrel{\delta_{g}}{\longrightarrow} \ldots
$$

Lemma $2.1\left(\check{C} \bullet\left(U_{\bullet}, \mathcal{M}\right), \delta_{g}\right)$ is a cochain complex.

Proof : We have to show that the square of $\delta_{g}$ vanishes. Let $\varphi \in \check{C}^{n-1}\left(U_{\bullet}, \mathcal{M}\right)$, then

$$
\begin{aligned}
\delta_{g} \delta_{g} \varphi & =\delta \delta \varphi+\delta\left(g^{\star} \varphi\right)+g^{\star} \delta \varphi+g^{\star}\left(g^{\star} \varphi\right) \\
& =\delta\left(g^{\star} \varphi\right)+g^{\star} \delta \varphi+g^{\star}\left(g^{\star} \varphi\right)
\end{aligned}
$$

and therefore

$$
\begin{aligned}
\left(\delta_{g} \delta_{g} \varphi\right)_{i_{0} \ldots i_{n+1}}= & \sum_{k=0}^{n+1}(-1)^{k}\left(g^{\star} \varphi\right)_{i_{0} \ldots \hat{i}_{k} \ldots i_{n+1}} \\
& +(-1)^{n}\left(\sum_{k=0}^{n}(-1)^{k} \varphi_{i_{0} \ldots \hat{i}_{k} \ldots i_{n}}-\sum_{k=0}^{n}(-1)^{k} \varphi_{i_{0} \ldots \hat{i}_{k} \ldots i_{n}} \cdot g_{i_{n} i_{n+1}}\right) \\
& +(-1)^{n}\left(g^{\star} \varphi\right)_{i_{0} \ldots i_{n}}-(-1)^{n}\left(g^{\star} \varphi\right)_{i_{0} \ldots i_{n}} \cdot g_{i_{n} i_{n+1}} \\
= & \sum_{k=0}^{n-1}(-1)^{k}(-1)^{n-1}\left(\varphi_{i_{0} \ldots \hat{i}_{k} \ldots i_{n}}-\varphi_{i_{0} \ldots \hat{i}_{k} \ldots i_{n}} \cdot g_{i_{n} i_{n+1}}\right) \\
& +(-1)^{n}(-1)^{n-1}\left(\varphi_{i_{0} \ldots i_{n-1}}-\varphi_{i_{0} \ldots i_{n-1}} \cdot g_{i_{n-1} i_{n+1}}\right) \\
& +(-1)^{n+1}(-1)^{n-1}\left(\varphi_{i_{0} \ldots i_{n-1}}-\varphi_{i_{0} \ldots i_{n-1}} \cdot g_{i_{n-1} i_{n}}\right) \\
& +(-1)^{n}\left(\sum_{k=0}^{n}(-1)^{k} \varphi_{i_{0} \ldots \hat{i}_{k} \ldots i_{n}}-\sum_{k=0}^{n}(-1)^{k} \varphi_{i_{0} \ldots \hat{i}_{k} \ldots i_{n}} \cdot g_{i_{n} i_{n+1}}\right) \\
& +(-1)^{n}(-1)^{n-1}\left(\varphi_{i_{0} \ldots i_{n-1}}-\varphi_{i_{0} \ldots i_{n-1}} \cdot g_{i_{n-1} i_{n}}\right) \\
& -(-1)^{n}(-1)^{n-1}\left(\varphi_{i_{0} \ldots i_{n-1}}-\varphi_{i_{0} \ldots i_{n-1}} \cdot g_{i_{n-1} i_{n}} \cdot g_{i_{n} i_{n+1}}\right) \\
= & 0 .
\end{aligned}
$$

${ }^{4}$ See A. 2 
The last equality holds due to the cocycle relation $g_{i j}+g_{j k}=g_{i k}$.

By the last lemma we have well-defined cohomology groups $\check{H}^{n}\left(U_{\bullet}, \mathcal{M}, g\right)$ for $n=0,1,2, \ldots$ and any open cover $U_{\bullet}=\left\{U_{i} \mid i \in I\right\}$ of $B$. And as in the untwisted case we define the twisted cohomology groups $\breve{H}^{n}(B, \mathcal{N}, g)$ of $B$ by passing to the limit

$$
\check{H}^{n}(B, \mathcal{M}, g):=\lim _{V_{\bullet}} \check{H}^{n}\left(V_{\bullet}, \mathcal{M}, g \mid\right),
$$

wherein the limit runs over all refinements $V_{\bullet}$ of $U_{\bullet}$. To be precise, consider a refinement $V_{\bullet}=\left\{V_{k} \mid k \in K\right\}$ of $U_{\bullet}=\left\{U_{i} \mid i \in I\right\}$ with refinement map $\iota: K \rightarrow I$, i.e. $V_{k} \subset U_{\iota(k)}$ then we define $\left(\iota^{*} g\right)_{k l}:=\left.g_{\iota(k) \iota(l)}\right|_{V_{k l}}$ and similarly $\left(\iota^{*} \varphi\right)_{k_{0} \ldots k_{n}}:=\varphi_{\iota\left(k_{0}\right) \ldots \iota\left(k_{n}\right)} \mid V_{k_{0} \ldots k_{n}}$ to obtain a cochain map $\iota^{*}:\left(\check{C}^{n}\left(U_{\bullet}, \mathcal{M}\right), \delta_{g}\right) \rightarrow$ $\left(\check{C}^{n}\left(V_{\bullet}, \mathcal{M}\right), \delta_{l^{*} g}\right)$. This construction defines a functor from the category of coverings with refinement maps as morphisms to the category of cochain complexes (and after taking homology to the category of graded abelian groups). The category of coverings is filtered in the following sense:

(i) Any two coverings have a common refinement, i.e. for any two objects $\left(U_{\bullet}, I\right)$ and $\left(V_{\bullet}, K\right)$ there is a third object $\left(W_{\bullet}, L\right)$ with morphisms $\left(U_{\bullet}, I\right) \rightarrow$ $\left(W_{\bullet}, L\right)$ and $\left(V_{\bullet}, K\right) \rightarrow\left(W_{\bullet}, L\right)$.

(ii) Any two refinement maps become equal finally, i.e. for any to morphisms $\iota:\left(U_{\bullet}, I\right) \rightarrow\left(V_{\bullet}, K\right)$ and $\kappa:\left(U_{\bullet}, I\right) \rightarrow\left(V_{\bullet}, K\right)$ there exists an object $\left(W_{\bullet}, L\right)$ and morphisms $\iota^{\prime}:\left(V_{\bullet}, K\right) \rightarrow\left(W_{\bullet}, L\right)$ and $\kappa^{\prime}:\left(V_{\bullet}, K\right) \rightarrow\left(W_{\bullet}, L\right)$ such that $\kappa^{\prime} \circ \kappa=\iota^{\prime} \circ \iota$.

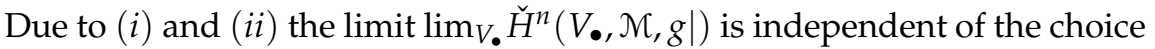
of the first covering and independent of the refinement maps.

So far, in our construction we referred explicitly to a choice of a cocycle $g \in \breve{Z}^{1}\left(U_{\bullet}, \mathcal{G}\right)$, but up to isomorphism $\breve{H}^{n}(B, \mathcal{M}, g)$ depends only on the class of $g$. In fact, let $V_{\bullet}=\left\{V_{k} \mid k \in K\right\}$ and $U_{\bullet}=\left\{U_{i} \mid i \in I\right\}$ be open coverings of $B$, and let $g^{\prime} \in \check{Z}^{1}\left(V_{\bullet}, \mathcal{G}\right)$ and $g \in \check{Z}^{1}\left(U_{\bullet}, \mathcal{G}\right)$ represent the same element in $\breve{H}^{1}(B, \mathcal{G})$. If $W_{\bullet}=\left\{W_{m} \mid m \in M\right\}$ is a common refinement with refinement maps $\iota: M \rightarrow I$ and $\kappa: M \rightarrow K$ then there are $r_{m} \in \mathcal{G}\left(W_{m}\right)$ such that $g_{\mathcal{\kappa}(m) \kappa(n)}^{\prime}\left|W_{m n}=r_{m}\right|_{W_{m n}}+g_{\iota(m) \iota(n)}\left|W_{m n}-r_{n}\right|_{W_{m n}}$. They give rise to the following diagram of cochain complexes

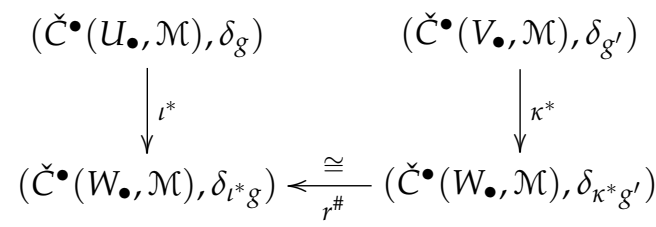

wherein $r^{\#}$ is defined by $\left(r^{\#} \varphi\right)_{k_{0} \ldots k_{n}}:=\left.\varphi_{k_{0} \ldots k_{n}} \cdot r_{k_{n}}\right|_{k_{k_{0} \ldots k_{n}}}$ for $\varphi \in \check{C}^{n}\left(V_{\bullet}, \mathcal{M}\right)$. One easily derives $\delta_{l^{*} g}\left(r^{\#} \varphi\right)=r^{\#} \delta_{\kappa^{*} g} \varphi$, for $\varphi \in \check{C}^{\bullet}\left(V_{\bullet}, \mathcal{M}\right)$, i.e. $r^{\#}$ is a cochain map and even an isomorphism. Thus the corresponding cohomology groups 
are isomorphic and this isomorphism passes to the limit. Therefore the twisted Čech groups $\check{H}^{n}(B, \mathcal{M},[g])$ are well defined for the class $[g] \in \check{H}^{1}(B, \mathcal{G})$ up to the considered isomorphism.

We now consider the relation between pairs and twisted Čech cohomology. One should note at this point that, just as in the untwisted case, the first Čech "group" $\check{H}^{1}(B, \mathcal{N}, g)$ is a well-defined set even in case the sheaf $\mathcal{N}$ is just a sheaf of groups and not necessarily abelian. In that case the additive (commutative) relation of being cohomologous $\zeta_{j i} \sim \zeta_{j i}+\left(\delta_{g} \eta\right)_{j i}$ is replaced by the multiplicative relation $\left.\left.\zeta_{j i} \sim \eta_{j}\right|_{U_{i j}} \cdot g_{j i} \zeta_{j i} \eta_{i}^{-1}\right|_{U_{i j}}$, for $\zeta \in \check{Z}^{1}\left(U_{\bullet}, \mathcal{M}, g\right), \eta \in \check{C}^{0}\left(U_{\bullet}, \mathcal{M}\right)$. The next proposition is then just a reformulation of what we already observed in Proposition 2.2.

Proposition 2.3 Let $B$ be a base space. Let $\mathcal{N}$ be the sheaf of topological groups on $B$ defined by $\mathcal{M}(U):=C(U, \operatorname{Map}(G / N, P U(\mathcal{H})))$, and let $\mathcal{G}:=G / N$, i.e. $G / N(U):=C(U, G / N)$, for $U \subset B . \underline{G / N}$ acts on $\mathcal{M}$ in the obvious way, i.e. by translation in the arguments. Then the first twisted cohomology classifies pairs over $B$, i.e. we have a bijection

$$
\check{H}^{1}(B, \mathcal{M}, g) \cong \operatorname{Par}(E, B),
$$

if the class $[g] \in \check{H}^{1}(B, G / N)$ is the class for the bundle $E$.

Proof : Let $g_{. .}, \zeta$.. be the transition functions of a pair over $B$ for an atlas $U_{\bullet}$. So $g \in \breve{Z}^{1}\left(U_{\bullet}, \underline{G / N}\right)$, and the crucial point is to observe that equation (6) is equivalent to $\delta_{g} \zeta=\mathbb{1}$ and therefore each pair defines an element in $\breve{H}^{1}(B, \mathcal{M}, g)$. In fact, this is well defined, because if $g_{. .}^{\prime} \zeta_{\text {.. }}^{\prime}$ are transition functions for another atlas then (after choosing a common refinement) the two classes match under the isomorphism $r^{\#}$, i.e. $r^{\#} \zeta^{\prime}$ is cohomologous to $\zeta$. Similarly, an isomorphism of pairs leads to cohomologous cocycles. Conversely, any $\zeta \in \check{Z}^{1}\left(U_{\bullet}, \mathcal{M}, g\right)$ defines an associated pair, and if $\zeta^{\prime} \in \check{Z}^{1}\left(V_{\bullet}, \mathcal{M}, g\right)$ defines the same class as $\zeta$.. then the two associated pairs are isomorphic. Since each class arises in such a way the assertion is proven.

Let $g_{. .}, \zeta$.. be the transition functions of a pair over $B$. Since $G / N$ is compact and $B$ paracompact we can apply Lemma A.7 and Lemma A.8 for the family of transition functions $\zeta_{i j}: U_{i j} \rightarrow \operatorname{Map}(G / N, \operatorname{PU}(\mathcal{H})$. I.e. we can find a refined atlas $\left\{V_{k} \mid k \in K:=I \times B\right\}, V_{k} \subset U_{i}$ if $k=(i, x)$, such that on its twofold intersections $V_{k l}$ the restricted transition functions lift to continuous functions $\bar{\zeta}_{k l}: V_{k l} \rightarrow \operatorname{Bor}(G / N, \mathrm{U}(\mathcal{H}))$. These lifts are unique up to continuous functions $V_{k l} \rightarrow \operatorname{Bor}(G / N, \mathrm{U}(1))$. Let us denote by $g_{l k}$ the restriction $\left.g_{j i}\right|_{V_{l k}}$ in case $l=(j, y), k=(i, x) \in I \times B$. On threefold intersections the function $V_{k l m} \ni u \mapsto \bar{\zeta}_{k l}(u)\left(g_{l m}(u)+_{-}\right)$won't be continuos as a function to $\operatorname{Bor}(G / N, U(\mathcal{H}))$ in general, but it will as a function to ${ }^{5} L^{\infty}(G / N, U(\mathcal{H}))$. So

${ }^{5} L^{\infty}(G / N, \mathrm{U}(\mathcal{H}))$ has the weak topology. See equation (51) in section A.3. 
equation (6) implies that there are continuous $\psi_{m l k}: V_{m l k} \rightarrow L^{\infty}(G / N, \mathrm{U}(1))$ such that

$$
\begin{aligned}
\bar{\zeta}_{m l}(u)\left(g_{l k}(u)+z\right) \bar{\zeta}_{l k}(u)(z) & =\bar{\zeta}_{m k}(u)(z) \psi_{m l k}(u)(z) \cdot \mathbb{1} \\
\Leftrightarrow \delta_{g} \bar{\zeta} & =\psi,
\end{aligned}
$$

and it follows that $\delta_{g} \psi=1$. The functions $\psi_{k l m}$ therefore define a twisted Čech 2-cocycle $\psi_{\ldots} \in \breve{Z}^{2}\left(V_{\bullet}, L^{\infty}(G / N, \mathrm{U}(1)), g\right)$.

Proposition 2.4 The construction of $\psi$... defines a homomorphism of groups

$$
\operatorname{Par}(E, B) \rightarrow \check{H}^{2}\left(B, \underline{L^{\infty}(G / N, \mathrm{U}(1))}, g\right)
$$

if $[g] \in \check{H}^{1}(B, \underline{G / N})$ is the class of the bundle $E \rightarrow B$.

Proof : We must check that the class $[\psi \ldots]$ is independent of all choices. In fact, if $\bar{\zeta}_{j i}$ and $\bar{\zeta}_{j i}^{\prime}$ are different choices of lifts of $\zeta_{j i}$, they differ by a scalar function

$s_{j i}=\bar{\zeta}_{j i}^{-1} \bar{\zeta}_{j i}^{\prime}$ and $\psi \delta_{g} s$ is the cocycle obtained from $\bar{\zeta}_{j i}^{\prime}$, so the class $[\psi]$ is not effected. It is also easy that the class does not change under the choice of the atlas or by considering a pair stably isomorphic to the first one.

We do not achieve the statement that the above homomorphism is injective or surjective, so we are far from classifying pairs by this map.

\subsection{Dynamical Triples and their Local Structure}

Let $P \rightarrow E \rightarrow B$ be a pair. The quotient map $G \ni g \mapsto g N \in G / N$ induces a $G$ action on $E$.

Definition 2.3 A decker is just a continuous action $\rho: P \times G \rightarrow P$ that lifts the induced G-action on $E$ such that $\rho(., g): P \rightarrow P$ is a bundle automorphism, for all $g \in G$.

The existence of deckers can be a very restrictive condition on the bundle $P \rightarrow E$. (See e.g. Prop. 2.6 below.) In fact, they need not exist and need not to be unique in general, but the play a central rôle in what follows, therefore we introduced an extra name.

In context of $C^{*}$-dynamical systems, i.e. $C^{*}$-algebras with (strongly continuous) group actions, concretely, in context of the equivariant Brauer group several notions of equivalence of actions occur [CKRW]. In particular, the notions of isomorphic actions, stably isomorphic actions and exterior equivalent actions are combined to the notion of stably outer conjugate actions. We slightly modify these notion for our purposes. However, we postpone the definition until we made ourselves familiar with the local structure of dynamical triples.

Definition 2.4 A dynamical triple $(\rho, P, E)$ over $B$ is a pair $(P, E)$ over $B$ together with a decker $\rho: P \times G \rightarrow P$. 
Let $(\rho, P, E)$ be a dynamical tripel over $B$.

Proposition $2.5 \quad$ i) If we define $\rho^{\tau}(g):=\rho(., g): P \rightarrow P$, we obtain a diagram of topolgical groups

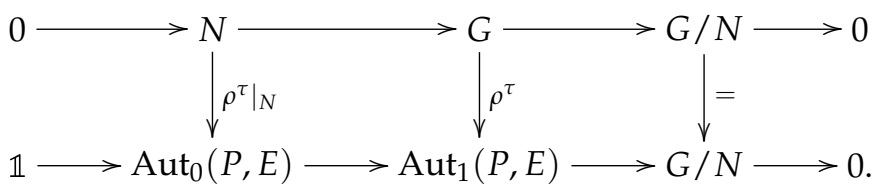

Conversely, if $B$ is locally compact then a commutative diagram (8) defines a decker.

ii) Locally, i.e. after choosing charts $U_{i}$, a decker defines a family of continuous cocycles $\mu_{i}: U_{i} \rightarrow Z_{\text {cont }}^{1}(G, \operatorname{Map}(G / N, \operatorname{PU}(\mathcal{H})))$ such that on twofold intersections $U_{i j} \ni u$ the transition functions of the pair and the cocycles are related by

$$
\mu_{i}(u)(g, z)=\zeta_{j i}(u)(z+g N)^{-1} \mu_{j}(u)\left(g, g_{j i}(u)+z\right) \zeta_{j i}(u)(z) .
$$

Conversely, any family of cocycles $\left\{\mu_{i}\right\}_{i \in I}$ that fulfils eq. (9) determines a unique decker.

Proof : i) The origin of the diagram is obvious. For the converse, it is sufficient to prove the result locally because the action of $G$ on $F$ preserves charts. Explicitly, over a chart $\left(U_{i}, k_{i}, h_{i}\right)$ the action of $g \in G$ is

$$
\begin{aligned}
h_{i}^{*}(\phi(g)): U_{i} \times G / N \times \mathrm{PU}(\mathcal{H}) & \rightarrow U_{i} \times G / N \times \mathrm{PU}(\mathcal{H}) . \\
(u, z, U) & \mapsto\left(u, g N+z, \mu_{i}^{\prime \prime}(g)(u, z) U\right)
\end{aligned}
$$

If $B$ is locally compact the exponential law (Lemma A.4) ensures that all functions $\mu_{i}^{\prime}:(u, g, z) \mapsto \mu_{i}^{\prime \prime}(g)(u, z)$ are jointly continuous.

ii) Locally, Lemma A.4 ensures that the transposed functions $\mu_{i}: U_{i} \rightarrow$ $\operatorname{Map}(G \times G / N, \operatorname{PU}(\mathcal{H}))$ made out of $\mu_{i}^{\prime}$ are well defined. The cocycle condition

$$
\mu_{i}(u)(g+h, z)=\mu_{i}(u)(g, z+h N) \mu_{i}(u)(h, z)
$$

and the validity of (9) are immediate as well as the converse statement.

It should be mentioned at this point that equation (9) (and its unitary version we consider later) is quite powerful as turns out. A first application is given in the next proposition. It is a complete answer to the existence of deckers in the case of $N=0$, i.e. $G=G / N$. The result is well-known, but we state a proof using (9) for the convenience of the reader. 
Proposition 2.6 Assume $N=0$ and let $P \stackrel{q}{\rightarrow} E \stackrel{p}{\rightarrow} B$ be a pair. Then a decker exists if and only if $P \cong p^{*} P^{\prime}$ for a $\mathrm{PU}(\mathcal{H})$-bundle $P^{\prime} \rightarrow B$.

Proof : If $P \cong p^{*} P^{\prime}$ for some $\mathrm{PU}(\mathcal{H})$-bundle $P^{\prime} \rightarrow B$ we obtain a decker by acting on the first entry of the fibered product $p^{*} P^{\prime}=E \times{ }_{B} P^{\prime}$.

Conversely, if a decker is given then we define a family $\zeta_{j i}^{\prime}: U_{j i} \rightarrow \mathrm{PU}(\mathcal{H})$ by $\zeta_{j i}^{\prime}(u):=\mu_{j}(u)\left(g_{j i}(u), 0\right)^{-1} \zeta_{j i}(u)(0)$, for $u \in U_{j i} \subset B$. This is well defined since $G=G / N$.

Claim $1:\left\{\zeta_{j i}^{\prime}\right\}_{j, i \in I}$ are transition functions for a $\mathrm{PU}(\mathcal{H})$-bundle $P^{\prime} \rightarrow B$.

Proof : Let $u \in U_{i} \cap U_{j} \cap U_{k}$. Then

$$
\begin{aligned}
& \zeta_{k j}^{\prime}(u) \zeta_{j i}^{\prime}(u) \\
= & \mu_{k}(u)\left(g_{k j}(u), 0\right)^{-1} \zeta_{k j}(u)(0) \mu_{j}(u)\left(g_{j i}(u), 0\right)^{-1} \zeta_{j i}(u)(0) \\
\stackrel{(9)}{=} & \underbrace{\mu_{k}(u)\left(g_{k j}(u), 0\right)^{-1} \mu_{k}(u)\left(g_{j i}(u), g_{k j}(u)+0\right)^{-1}}_{\text {cocy.cond. }} \underbrace{\zeta_{k j}(u)\left(0+g_{j i}(u)\right) \zeta_{j i}(u)(0)}_{\mu_{k}(u)\left(g_{j i}(u)+g_{k j}(u), 0\right)^{-1} \stackrel{(6)}{=} \zeta_{k i}(u)(0)} \\
= & \zeta_{k i}^{\prime}(u) .
\end{aligned}
$$

Claim 2: $P \cong p^{*} P^{\prime}$.

Proof : The bundle $q^{\prime}: p^{*} P^{\prime} \rightarrow E$ has transition functions

$$
p^{*} \zeta_{j i}^{\prime}: p^{-1}\left(U_{i j}\right) \cong U_{i j} \times G / N \ni(u, z) \mapsto \zeta_{j i}^{\prime}(u) .
$$

We define an isomorphism $f: P \rightarrow p^{*} P^{\prime}$ locally $f_{i}:=\left.f\right|_{q^{-1}\left(p^{-1}\left(U_{i}\right)\right)}$ by

$$
\begin{array}{rlc}
P \supset q^{-1}\left(p^{-1}\left(U_{i}\right)\right. & \stackrel{f_{i}}{\longrightarrow} & q^{\prime-1}\left(p^{-1}\left(U_{i}\right)\right) \subset p^{*} P^{\prime} \\
\downarrow \cong & & \downarrow \cong \\
U_{i} \times G / N \times \mathrm{U}(\mathcal{H}) & \longrightarrow & U_{i} \times G / N \times \mathrm{U}(\mathcal{H}) \\
(u, z, U) & \longmapsto & \left(u, z, \mu_{i}(u)(z, 0)^{-1} U\right) .
\end{array}
$$

This is in fact a well defined global isomorphism since form eq. (9) it follows for $G=G / N$ and $u \in U_{i} \cap U_{j}$

$$
\begin{aligned}
\mu_{i}(u)(z, 0)^{-1} & =\zeta_{j i}(u)(0)^{-1} \mu_{j}(u)\left(z, g_{j i}(u)\right)^{-1} \zeta_{j i}(u)(z) \\
& =\zeta_{j i}(u)(0)^{-1} \mu_{j}\left(g_{j i}(u)\right)(u, 0) \mu_{j}(u)\left(z+g_{j i}(u), 0\right)^{-1} \zeta_{j i}(u)(z) \\
& =\zeta_{j i}^{\prime}(u)^{-1} \mu_{j}(u)\left(z+g_{j i}(u), 0\right)^{-1} \zeta_{j i}(u)(z) .
\end{aligned}
$$

Thus the local definition of $f$ is independent of the chosen chart.

We now introduce the notions of equivalence we mentioned earlier. 
Definition 2.5 Two deckers $\rho, \rho^{\prime}: G \times P \rightarrow P$ on a pair $(P, E)$ are called exterior equivalent if the continuous function $c: P \times G \rightarrow P$ which is defined by $\rho(.,-g) \circ$ $\rho^{\prime}(., g)=c(., g): P \rightarrow P$, for $g \in G$, is locally of the following form: There

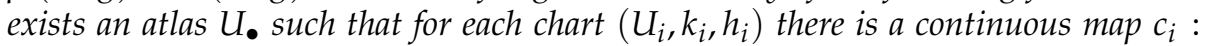
$U_{i} \times G \times G / N \rightarrow \mathrm{U}(\mathcal{H})$ which satisfies the three conditions ${ }^{6}$

(E0) $\left(h_{i}^{-1} \circ c(-, g) \circ h_{i}\right)(u, z, U)=\left(u, z, \operatorname{Ad}\left(c_{i}(u, g, z)\right) U\right) \in U_{i} \times G / N \times \operatorname{PU}(\mathcal{H})$,

(E1) $c_{j}\left(u, g, g_{j i}(u)+z\right)=\zeta_{j i}(u)(z)\left(c_{i}(u, g, z)\right) \in \mathrm{U}(\mathcal{H})$ and

(E2) $c_{i}(u, h+g, z)=\mu_{i}(u)(g, z)^{-1}\left(c_{i}(u, h, z+g N)\right) c_{i}(u, g, z) \in \mathrm{U}(\mathcal{H})$,

for the transition functions $g_{j i}, \zeta_{j i}$ of the pair and the cocycles $\mu_{i}$ of the decker $\rho$ as above.

It is clear that, if one has given a family of continuous unitary functions $\left\{c_{i}\right\}$ which satisfies (E0), (E1) and (E2) for the cocycles $\left\{\mu_{i}\right\}$ of a decker $\rho$, the family of cocycles

$$
\mu_{i}^{\prime}(u)(g, z):=\mu_{i}(u)(g, z) \operatorname{Ad}\left(c_{i}(u, g, z)\right)
$$

defines an exterior equivalent decker $\rho^{\prime}$ to $\rho$.

Let $c^{\tau}: G \rightarrow \operatorname{Aut}_{0}(P, E)$ be defined by $c^{\tau}(g):=c(., g)$. It satisfies the cocycle condition

$$
c^{\tau}(g+h)=c^{\tau}(g) \cdot \rho^{\tau}(h) c^{\tau}(h),
$$

where $\cdot$ is the right action of $\operatorname{Aut}_{1}(P, E)$ on $\operatorname{Aut}_{0}(P, E)$ given by conjugation. This right action lifts to the sections $\Gamma\left(E, P \times_{\mathrm{PU}(\mathcal{H})} \mathrm{U}(\mathcal{H})\right)$ of the to $P$ associated $\mathrm{U}(\mathcal{H})$-bundle in the following diagram, i.e. the vertical map is $\operatorname{Aut}_{1}(P, E)$ equivariant,

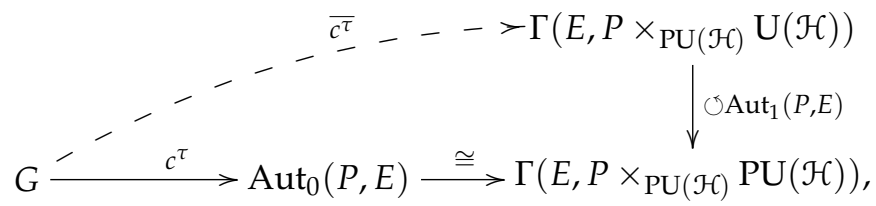

Therein the associated bundles are both obtained by the the conjugate action of $\mathrm{PU}(\mathcal{H})$ on the respective groups. Now, conditions (E0) - (E2) imply that we can lift $c^{\tau}$ to a unitary cocycle $\overline{\mathcal{c}^{\tau}}: G \rightarrow \Gamma\left(E, P \times_{\mathrm{PU}(\mathcal{H})} \mathrm{U}(\mathcal{H})\right)$, i.e.

$$
\overline{c^{\tau}}(g+h)=\overline{c^{\tau}}(g) \cdot \rho^{\tau}(h) \overline{c^{\tau}}(h)
$$

Similar to Proposition 2.5 this global statement is an equivalent formulation of exterior equivalence if the base $B$ is locally compact.

Proposition 2.7 Let B be locally compact, and let $\rho$ and $\rho^{\prime}$ be deckers on a pair $(P, E)$. These two deckers are exterior equivalent if and only if $c^{\tau}$ as defined above lifts to a unitary cocycle $\overline{c^{\tau}}$.

\footnotetext{
${ }^{6}$ Here and in what follows we will always use the notation $\zeta(c), \zeta \in \mathrm{PU}(\mathcal{H}), c \in \mathrm{U}(\mathcal{H})$, for the action of $\mathrm{PU}(\mathcal{H})$ on $\mathrm{U}(\mathcal{H})$ by conjugation.
} 
We do not give a detailed proof of this fact, it is again just an application of the exponential law for locally compact spaces.

The next statement gives an important example of exterior equivalent deckers.

Example 2.1 Let $\rho$ be a decker on an arbitrary pair $(P, E)$, and let $v: P \rightarrow P$ be a bundle automorphism. Then the conjugate decker $\rho^{v}$ is exterior equivalent to $\rho$ if the class $[v] \in \check{H}^{1}(E, \mathrm{U}(1))$ of the automorphism vanishes.

Proof : Let us denote by $\mu_{i}$ the cocycles of the decker $\rho$ which satisfy (9) on a chosen atlas $\left\{U_{i}\right\}_{i \in I}$. Because the class of $v$ vanishes, we can assume without restriction that it is locally implemented by unitary functions $v_{i}: U_{i} \rightarrow$ $\operatorname{Map}(G / N, \mathrm{U}(\mathcal{H}))$ such that $\zeta_{j i}(u)(z)\left(v_{i}(u)(z)\right)=v_{j}(u)\left(g_{j i}(u)+z\right)$.

Locally, $\rho\left({ }_{-},-g\right) \circ \rho^{v}\left(_{-}, g\right)$ is given by

$$
\begin{aligned}
& \mu_{i}(u)(-g, z+g N) \operatorname{Ad}\left(v_{i}(u)(z+g N)\right) \mu_{i}(u)(g, z) \operatorname{Ad}\left(v_{i}(u)(z)^{-1}\right) \\
= & \mu_{i}(u)(g, z)^{-1} \operatorname{Ad}\left(v_{i}(u)(z+g N)\right) \mu_{i}(u)(g, z) \operatorname{Ad}\left(v_{i}(u)(z)^{-1}\right) \\
= & \operatorname{Ad}\left(c_{i}(u, g, z)\right)
\end{aligned}
$$

for $c_{i}(u, g, z):=\mu_{i}(u)(g, z)^{-1}\left(v_{i}(u)(z+g N)\right) v_{i}(u)(z)^{-1} \in \mathrm{U}(\mathcal{H})$. So condition (E0) is satisfied. We check that the conditions (E1) and (E2) also holds. In fact,

$$
\begin{array}{ll} 
& \zeta_{j i}(u)(z)\left(c_{i}(u, g, z)\right) \\
= & \left.\zeta_{j i}(u)(z)\left(\mu_{i}(u)(g+h, z)^{-1}\right)\left(v_{i}(u)(z+g N+h N)\right) v_{i}(u)(z)^{-1}\right) \\
\stackrel{(9)}{=} & \mu_{j}(u)\left(g, g_{j i}(u)+z\right)^{-1}\left(\zeta_{j i}(u)(z+g N)\left(v_{i}(u)(z+g N)\right)\right) \zeta_{j i}(u)(z)\left(v_{i}(u)(z)\right)^{-1} \\
\stackrel{[v]=1}{=} & c_{j}(u)\left(g, g_{j i}(u)+z\right)
\end{array}
$$

which proves (E1), and

$$
\begin{aligned}
& c_{i}(u, g+h, z) \\
= & \mu_{i}(u)(g+h, z)^{-1}\left(v_{i}(u)(z+g N+h N)\right) v_{i}(u)(z)^{-1} \\
= & \mu_{i}(u)(g, z)^{-1}\left(\mu_{i}(u)(h, z+g N)^{-1}\left(v_{i}(u)(z+g N+h N)\right)\right) v_{i}(u)(z)^{-1} \\
= & \mu_{i}(u)(g, z)^{-1}\left(\mu_{i}(u)(h, z+g N)^{-1}\left(v_{i}(u)(z+g N+h N)\right) v_{i}(u)(z+g N)\right) \\
& \mu_{i}(u)(g, z)^{-1}\left(v_{i}(u)(z+g N)\right) v_{i}(u)(z)^{-1}
\end{aligned}
$$

which proves (E2).

Between two dynamical triples we introduce a notion of equivalence based on exterior equivalence.

Two dynamical triples $(\rho, P, E)$ and $\left(\rho^{\prime}, P^{\prime}, E^{\prime}\right)$ are isomorphic if there is a morphism $(\varphi, \vartheta, \theta)$ of the underlying pairs such that $\rho=\vartheta^{*} \rho^{\prime}$. The triples 
are outer conjugate if there is a morphism $(\varphi, \vartheta, \theta)$ of the underlying pairs such that $\rho$ and $\vartheta^{*} \rho^{\prime}$ are exterior equivalent on $(P, E)$. Furthermore, we call the triples stably isomorphic (respectively stably outer conjugate) if the triples $(\mathbb{1} \otimes \rho, \mathrm{PU}(\mathcal{H}) \otimes P, E)$ and $\left(\mathbb{1} \otimes \rho^{\prime}, \mathrm{PU}(\mathcal{H}) \otimes P^{\prime}, E^{\prime}\right)$ are isomorphic (resp. outer conjugate).

We can arrange these notions in a diagram of implications.

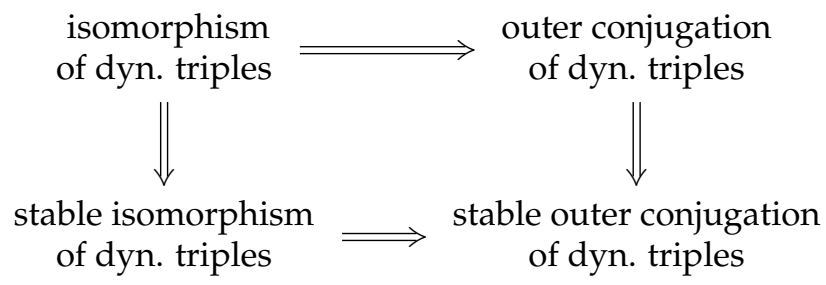

The following example shall illustrate an important feature of the notion of stably outer conjugation.

Example 2.2 Let $(\rho, P, E)$ be a dynamical triple, and let $\lambda_{G}: G \rightarrow \mathrm{U}\left(L^{2}(G)\right)$ be the left regular representation of $G$. Then the two triples $(\rho, P, E)$ and $\left(\left(\operatorname{Ad} \circ \lambda_{G}\right) \otimes\right.$ $\left.\rho, \mathrm{PU}\left(L^{2}(G)\right) \otimes P, E\right)$ are stably outer conjugate.

Proof: The triple $(\rho, P, E)$ and its stabilisation $\left(\mathbb{1} \otimes \rho, \operatorname{PU}\left(L^{2}(G)\right) \otimes P, E\right)$ are stably isomorphic and the triples $\left(\mathbb{1} \otimes \rho, \mathrm{PU}\left(L^{2}(G)\right) \otimes P, E\right)$ and $\left(\operatorname{Ad} \circ \lambda_{G} \otimes\right.$ $\left.\rho, \mathrm{PU}\left(L^{2}(G)\right) \otimes P, E\right)$ are exterior equivalent by $c_{i}(u, g, z):=\lambda_{G}(g) \otimes \mathbb{1}_{\mathcal{H}}$.

By Dyn we denote the set valued functor that sends a base space $B$ to the set of equivalence classes of stably outer conjugate dynamical triples over it, i.e.

$$
\operatorname{Dyn}(B):=\{\text { dynamical triples over } B\} / \text { stable outer conj. }
$$

In the same manner as we did for the functor Par we can fix a bundle $E \rightarrow B$ and define

$$
\operatorname{Dyn}(E, B):=\{\text { dynamical triples over } B \text { with fixed } E\} / \text { stable outer conj. over } \operatorname{id}_{E} \text {. }
$$

Isomorphic bundles $E, E^{\prime}$ lead to isomorphic sets $\operatorname{Dyn}(E, B) \cong \operatorname{Dyn}\left(E^{\prime}, B\right)$, and the bundle automorphisms $\operatorname{Aut}_{B}(E)$ act on $\operatorname{Dyn}(E, B)$ by pullback. This yields a decomposition $\operatorname{Dyn}(B) \cong \coprod_{[E]}\left(\operatorname{Dyn}(E, B) / \operatorname{Aut}_{B}(E)\right)$.

Our next goal is to find the link between dynamical triples and the cohomology theory we introduce now. Let $\mathcal{M}^{n}$ be the abelian sheaf on $B$ defined by $\mathcal{M}^{n}(U):=C\left(U, \operatorname{Bor}\left(G^{\times n}, L^{\infty}(G / N, \mathrm{U}(1))\right)\right)$, for $n=0,1,2, \ldots$ Let $U_{\bullet}=\left\{U_{i} \mid i \in I\right\}$ be an open cover of $B$ and let $g \in Z^{1}\left(U_{\bullet}, \underline{G / N}\right)$. Note that $\mathcal{M}^{n}$ is a right $G / N$-module, for all $n=0,1,2, \ldots$, by shifting the $G / N$-variable. We 
consider the the double complex

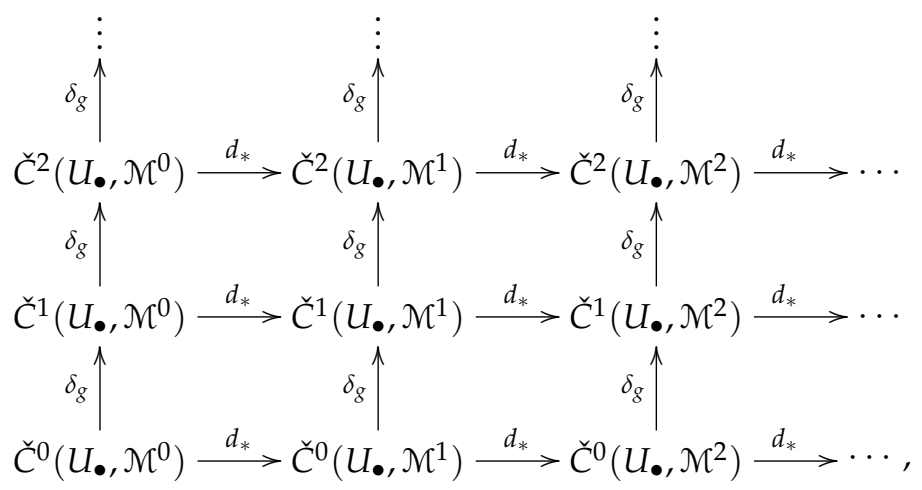

wherein the horizontal arrows $d_{*}$ are induced by the boundary operator $d$ : $\operatorname{Bor}\left(G^{\times n}, L^{\infty}(G / N, \mathrm{U}(1))\right) \rightarrow \operatorname{Bor}\left(G^{\times n+1}, L^{\infty}(G / N, \mathrm{U}(1))\right)$ of group cohomol$\operatorname{ogy}^{7}$ by acting point-wise on functions and the vertical arrows $\delta_{g}$ are the twisted Čech coboundary operators. In fact, all of the squares are commutative, hence we obtain a resulting total complex $\left(C_{\text {tot }}^{\bullet}\left(U_{\bullet}, \mathcal{M} \mathcal{M}^{\bullet}\right), \partial_{g}\right)$, i.e. $C_{\text {tot }}^{p}\left(U_{\bullet}, \mathcal{M} \bullet\right):=$ $\bigoplus_{p=k+l} \breve{C}^{k}\left(U_{\bullet}, \mathcal{M}^{l}\right)$ and $\left.\partial_{g}\right|_{C_{\text {tot }}^{p}\left(U_{\bullet}, \mathcal{M} \cdot\right)}:=\delta_{g}-(-1)^{p} d_{*}$ define a cochain complex. The choice of the sign in $\partial_{g}$ will be convenient. By $H_{\text {tot }}^{\bullet}\left(U_{\bullet}, \mathcal{M}^{\bullet}, g\right)$ we denote the corresponding cohomology groups, and by passing to the limit over all refinements of the open covering $U_{\bullet}$ we obtain

$$
H_{\text {tot }}^{\bullet}(B, \mathcal{M} \bullet, g):=\lim _{V_{\bullet}} H_{\text {tot }}^{\bullet}\left(V_{\bullet}, \mathcal{M}^{\bullet}, g\right) .
$$

We call this group the total cohomology of $B$ with twist $g$. In the same manner as explained on page 21 the limit does not depend on the covering $U_{\bullet}$ and is independent of the choice of the refinement maps.

It is also similar to the discussion on twisted Čech cohomology that the total cohomology groups are well defined objects for the class $[g]$ of a cocycle $g$ up to an isomorphism.

The connexion of dynamical triples and total cohomology has its origin in the local structure of triples as we explain now. Let $(\rho, P, E)$ be a dynamical triple. Let $U_{0}=\left\{U_{i} \mid i \in I\right\}$ be an atlas for the underlying pair with transition functions $g_{i j}, \zeta_{i j}$ and continuous cocycles $\mu_{i}$ as in Proposition 2.5. One should realise at this point that when we suppress the non-commutativity of $\mathrm{PU}(\mathcal{H})$ for a moment the equations $\delta_{g} \zeta_{. .}=\mathbb{1}, d\left(\mu_{i}(u)\right)=\mathbb{1}$ and equation (9) are equivalent to $\partial_{g}\left(\zeta . ., \mu_{.}\right)=\mathbb{1}$. We lift the transition functions and the cocycles to Borel functions. This will define a 2-cocycle for the total cohomology of $B$; in detail: Without restriction (see equation (7)) we can assume that the atlas is chosen such that the transition functions can be lifted continuously to Borel valued

${ }^{7}$ See A.2 
functions $\bar{\zeta}_{i j}$; they define a twisted Čech 2-cocycle $\delta_{g} \bar{\zeta}=: \psi_{\ldots} \in \check{C}^{2}\left(U_{\bullet}, \mathcal{M}^{0}\right)$. Further we can assume (if necessary we refine the atlas once more) that all charts are contractible. Therefore we can apply Corollary A.2 to each of the $\mu_{i}$ and obtain continuous functions $\bar{\mu}_{i}: U_{i} \rightarrow \operatorname{Bor}(G \times G / N, \mathrm{U}(\mathcal{H}))$ lifting $\mu_{i}$, for all $i \in I$. By Lemma A. 2 we can also pass to $\bar{\mu}_{i}: U_{i} \rightarrow \operatorname{Bor}\left(G, L^{\infty}(G / N, \mathrm{U}(\mathcal{H}))\right)$ which is important to as $L^{\infty}(G / N, \mathrm{U}(\mathcal{H}))$ is a continuous $G / N$-module. From the cocycle identity $d\left(\mu_{i}(u)\right)=\mathbb{1}$ we see that $d\left(\bar{\mu}_{i}(u)\right)=: \omega_{i}(u)$ defines a group cohomology 2-cocycle $\omega . \in \check{C}^{0}\left(U_{\bullet}, \mathcal{M}^{2}\right)$. On twofold intersections we can define $\phi_{j i}(u)(g, z):=\bar{\mu}_{i}(u)(g, z) \bar{\zeta}_{j i}(u)(z)^{-1} \bar{\mu}_{j}(u)\left(g, g_{j i}(u)+z\right)^{-1} \bar{\zeta}_{j i}(u)(z+g N)$ which is due to equation (9) $\mathrm{U}(1)$-valued, i.e. $\phi . . \in \breve{C}^{1}\left(U_{\bullet}, \mathcal{M}^{1}\right)$. Now, the three families of functions $\psi_{\ldots . .}, \phi$.. and $\omega$. satisfy the algebraic relations

$$
\begin{aligned}
\delta_{g} \psi & =1 \\
\delta_{g} \phi & =d_{*} \psi \\
d_{*} \phi & =\delta_{g} \omega \\
d_{*} \omega & =1
\end{aligned}
$$

which is equivalent to

$$
\partial_{g}\left(\psi_{\ldots,}, \phi_{. .,} \omega_{.}\right)=0 \in C_{\text {tot }}^{3}\left(U_{\bullet}, \mathcal{M}^{\bullet}\right),
$$

i.e. $\left(\psi_{\ldots, \ldots}, \phi_{. .}, \omega_{.}\right)$is a total 2 -cocycle. Of course, one can verify this by direct computation, but indeed it is implicitly clear, because, informally ${ }^{8}$, we have defined $\left(\psi_{. . .}, \phi_{. .}, \omega_{.}\right):=\partial_{g}\left(\bar{\zeta}_{. .}, \bar{\mu}.\right) \in C_{\text {tot }}^{2}\left(U_{\bullet}, \mathcal{M} \bullet\right)$.

Proposition 2.8 The assignment $(\rho, P, E) \mapsto\left(\psi \ldots, \phi_{. .}, \omega_{.}\right)$constructed above defines a homomorphism of groups

$$
\operatorname{Dyn}(E, B) \rightarrow H_{\text {tot }}^{2}\left(B, \mathcal{M} \mathcal{M}^{\bullet}, g_{. .}\right) .
$$

Proof : We must check that the defined total cohomology class is independent of all choices. This is simple to verify for the choice of the atlas, and the choice of the lifts of the transition functions and cocycles. As stably isomorphic pairs have the same local description, it is also clear that stably isomorphic pairs define the same total cohomology class. In detail we give the calculation that exterior equivalent triples define the same class:

Let $\rho$ and $\rho^{\prime}$ be exterior equivalent deckers on $(P, E)$. Let $c_{i}: U_{i} \times G \times$ $G / N \rightarrow \mathrm{U}(\mathcal{H})$ be such that (E0), (E1) and (E2) of Defintion 2.5 are satisfied. If $\mu_{i}$ and $\mu_{i}^{\prime}$ are the cocycles for the deckers, they both satisfy (9) for the same family of transition functions $\zeta_{j i}$. They are related by $\mu_{i}^{\prime}=\mu_{i}\left(\operatorname{Ad} \circ c_{i}\right)$, and if $\bar{\mu}_{i}$ is a lift for $\mu_{i}$, then $\bar{\mu}_{i}^{\prime}:=\bar{\mu}_{i} c_{i}$ defines a lift for $\mu_{i}^{\prime}$. Let $\left(\psi_{\ldots,}, \phi_{. .}, \omega\right.$.) be the

\footnotetext{
${ }^{8}$ i.e. up to the non-comutativity of $U(\mathcal{H})$
} 
cocycle obtained from $\left(\bar{\zeta}_{j i}, \bar{\mu}_{i}\right)$. Then we have

$$
\begin{aligned}
& \bar{\mu}_{i}^{\prime}(u)(h+g, z) \\
= & \bar{\mu}_{i}(u)(h+g, z) c_{i}(u, h+g, z) \\
= & \bar{\mu}_{i}(u)(h, z+g N) \bar{\mu}_{i}(u)(h, z) c_{i}(u, h+g, z) \omega_{i}(u)(g, h, z) \\
\stackrel{\text { (E2) }}{=} & \bar{\mu}_{i}(u)(h, z+g N) \bar{\mu}_{i}(u)(g, z) \mu_{i}(u)(g, z)^{-1}\left(c_{i}(u, h, z+g N)\right) c_{i}(g, z) \omega_{i}(u)(g, h, z) \\
= & \bar{\mu}_{i}(u)(h, z+g N) c_{i}(u, h, z+g N) \bar{\mu}_{i}(u)(g, z) c_{i}(g, z) \omega_{i}(u)(g, h, z) \\
= & \bar{\mu}_{i}^{\prime}(u)(h, z+g N) \bar{\mu}_{i}^{\prime}(u)(g, z) \omega_{i}(u)(g, h, z),
\end{aligned}
$$

so $\omega_{i}^{\prime}=\omega_{i}$, and

$$
\begin{aligned}
& \bar{\mu}_{i}^{\prime}(u)(g, z) \\
= & \bar{\mu}_{i}(u)(g, z) c_{i}(u, g, z) \\
= & \bar{\zeta}_{j i}(u)(z+g N)^{-1} \bar{\mu}_{j}(u)\left(g, g_{j i}(u)+z\right) \bar{\zeta}_{j i}(u)(z) c_{i}(u, g, z) \phi_{j i}(u)(g, z) \\
\stackrel{\text { (E1) }}{=} & \bar{\zeta}_{j i}(u)(z+g N)^{-1} \bar{\mu}_{j}(u)\left(g, g_{j i}(u)+z\right) c_{j}\left(u, g, g_{j i}(u)+z\right) \bar{\zeta}_{j i}(u)(z) \phi_{j i}(u)(g, z) \\
= & \bar{\zeta}_{j i}(u)(z+g N)^{-1} \bar{\mu}_{j}^{\prime}(u)\left(g, g_{j i}(u)+z\right) \bar{\zeta}_{j i}(u)(z) \phi_{j i}(u)(g, z),
\end{aligned}
$$

so $\phi_{j i}^{\prime}=\phi_{j i}$.

\subsection{Dual Pairs and Triples}

Of course, the whole discussion we made so far for $(G, N)$ can be done for $\left(\widehat{G}, N^{\perp}\right)$. I.e. we can replace $G$ by its dual group $\widehat{G}:=\operatorname{Hom}(G, U(1))$ and $N$ by the annihilator $N^{\perp}:=\left\{\chi|\chi|_{N}=1\right\} \subset \widehat{G}$ of $N$ everywhere. This is meaningful as $\widehat{G}$ is second countable, $N^{\perp}$ is discrete and $\widehat{G} / N^{\perp}$ compact (see A.1).

Definition 2.6 Let B be a base space.

i) A dual pair $(\widehat{P}, \widehat{E})$ over $B$ with underlying Hilbert space $\mathcal{H}$ is a sequence $\widehat{P} \rightarrow$ $\widehat{E} \rightarrow B$, wherein $\widehat{E} \rightarrow B$ is a $\widehat{G} / N^{\perp}$-principal fibre bundel and $\widehat{P} \rightarrow \widehat{E} a$ $\mathrm{PU}(\mathcal{H})$-principal fibre bundle, such that the latter bundle is already trivial over the fibres of $\widehat{E} \rightarrow B$.

ii) A dual decker $\hat{\rho}$ is an action $\hat{\rho}: \widehat{P} \times \widehat{G} \rightarrow \widehat{P}$ that lifts the induced $\widehat{G}$ action on $\widehat{E}$ and $\hat{\rho}\left({ }_{-}, \chi\right): \widehat{P} \rightarrow \widehat{P}$ is a bundle isomorphisms for all $\chi \in \widehat{G}$.

iii) A dual dynamical triple $(\hat{\rho}, \widehat{P}, \widehat{E})$ over $B$ is a pair $(\widehat{P}, \widehat{E})$ over B equipped with a dual decker $\hat{\rho}$

It is clear now how we define $\widehat{\operatorname{Par}}(B), \widehat{\operatorname{Par}}(\widehat{E}, B), \widehat{\operatorname{Dyn}}(B), \widehat{\operatorname{Dyn}}(\widehat{E}, B)$ and how all statements we have achieved so far translate to dual pairs and triples. 


\subsection{Topological Triples}

We introduce topological triples built out of a pair and a dual one. They were introduced first in [BRS] under the name T-duality triples in the special case $G=\mathbb{R}^{n}, N=\mathbb{Z}$. Our definition won't be exactly the same as in [BRS] - we comment on this in section 3.3.

There is a canonical $U(1)$-principal fibre bundle over $G / N \times \widehat{G} / N^{\perp}$ which is called Poincaré bundle. We recall its definition. Let

$$
Q:=(G / N \times \widehat{G} \times \mathrm{U}(1)) / N^{\perp},
$$

where the action of $N^{\perp}$ is defined by $(z, \chi, t) \cdot n^{\perp}:=\left(z, \chi+n^{\perp}, t\left\langle n^{\perp}, z\right\rangle^{-1}\right)$. Then the obvious map $Q \rightarrow G / N \times \widehat{G} / N^{\perp}$ is a $\mathrm{U}(1)$-principal fibre bundle. Indeed, $\mathrm{U}(1)$ acts freely and transitive in each fibre by multiplication in the third component and local sections of $Q \rightarrow G / N \times \widehat{G} / N^{\perp}$ are given by $G / N \times$ $V_{a} \ni(z, \hat{z}) \mapsto\left[\left(z, \hat{s}_{a}(\hat{z}), 1\right)\right] \in Q$, where, by Lemma A.1, $\hat{s}_{a}: V_{a} \rightarrow \widehat{G}$ is a family of local sections of the $N^{\perp}$-principal bundle $\widehat{G} \rightarrow \widehat{G} / N^{\perp}, V_{a} \subset \widehat{G} / N^{\perp}$. On the overlap $G / N \times V_{a b}$ two such sections are related by

$$
\begin{aligned}
{\left[\left(z, \hat{s}_{a}(\hat{z}), 1\right)\right] } & =\left[\left(z, \hat{s}_{b}(\hat{z})-n_{a b}^{\perp}(\hat{z}), 1\right)\right] \\
& =\left[\left(z, \hat{s}_{b}(\hat{z}),\left\langle n^{\perp}(\hat{z}), z\right\rangle\right)\right] \\
& =\left[\left(z, \hat{s}_{b}(\hat{z}), 1\right)\right] \cdot\left\langle n^{\perp}(\hat{z}), z\right\rangle,
\end{aligned}
$$

wherein $n_{a b}^{\perp}(\hat{z}):=-\hat{s}_{a}(\hat{z})+\hat{s}_{b}(\hat{z})$ which defines a family of transition functions $n_{a b}^{\perp}: V_{a b} \rightarrow N^{\perp}$ for the bundle $\widehat{G} \rightarrow \widehat{G} / N^{\perp}$. Thus we have found that $v_{a b}^{\perp}$ : $G / N \times V_{a b} \rightarrow \mathrm{U}(1)$ defined by $v_{a b}^{\perp}(z, \hat{z}):=\left\langle n_{a b}^{\perp}(\hat{z}), z\right\rangle$ are transition functions for the bundle $Q$.

Dually, there is a second $\mathrm{U}(1)$-bundle $R:=\left(G \times \widehat{G} / N^{\perp} \times \mathrm{U}(1)\right) / N \rightarrow$ $G / N \times \widehat{G} / N^{\perp}$ which has transition functions $v_{c d}: W_{c d} \times \widehat{G} / N^{\perp} \rightarrow \mathrm{U}(1)$ defined by $v_{c d}(z, \hat{z}):=\left\langle\hat{z}, n_{c d}(z)\right\rangle$ for an open cover $\left\{W_{a}\right\}$ of $G / N$ and transition functions $n_{c d}: W_{c d} \rightarrow N$ of $G \rightarrow G / N$.

We denote the Čech classes in $\check{H}^{1}\left(G / N \times \widehat{G} / N^{\perp}, \mathrm{U}(1)\right)$ which these bundles define by $[Q]$ and $[R]$.

Definition 2.7 The class $\pi:=-[Q]$ constucted above is called the Poincare class of $G / N \times \widehat{G} / N^{\perp}$.

Of course, in this definition we made a choice, but up to a sign there is none.

Lemma 2.2 $[Q]$ and $[R]$ are inverses of each other, i.e. $[Q]+[R]=0 \in \check{H}^{1}(G / N \times$ $\left.\widehat{G} / N^{\perp}, \underline{U(1)}\right)$.

Proof : We have to show that $\left\{v_{c d} v_{a b}^{\perp}: W_{c d} \times V_{a b} \rightarrow \mathrm{U}(1)\right\}_{(a, c),(b, d)}$ is a Čech coboundary. Let $\hat{s}_{a}: V_{a} \rightarrow \widehat{G}$ and $s_{c}: W_{c} \rightarrow G$ be families of local sections such that $n_{c d}(z)=-s_{c}(z)+s_{d}(z)$ and $n_{a b}^{\perp}(\hat{z})=-\hat{s}_{a}(\hat{z})+\hat{s}_{b}(\hat{z})$. We show that

$\left\{v_{c d} v_{a b}^{\perp}: W_{c d} \times V_{a b} \rightarrow \mathrm{U}(1)\right\}_{(a, c),(b, d)}=\delta\left(\left\{\left\langle\hat{s}_{a}(. .), s_{c}(-)\right\rangle: W_{c} \times V_{a} \rightarrow \mathrm{U}(1)\right\}_{(a, c)}\right)$, 
wherin $\delta$ is the usual Čech coboundary operator. It is $\left\langle\hat{z}, n_{c d}(z)\right\rangle=\left\langle\hat{s}_{a}(\hat{z}), n_{c d}(z)\right\rangle$ and $\left\langle n_{a b}^{\perp}(\hat{z}), z\right\rangle=\left\langle n_{a b}^{\perp}(\hat{z}), s_{d}(z)\right\rangle$, because $n_{c d}(z) \in N$ and $n_{a b}^{\perp}(\hat{z}) \in N^{\perp}$; therein the right hand side is the pairing $G \times \widehat{G} \rightarrow \mathrm{U}(1)$. Thus

$$
\begin{aligned}
v_{c d}(z, \hat{z}) v_{a b}^{\perp}(z, \hat{z}) & =\left\langle\hat{s}_{a}(\hat{z}), n_{c d}(z)\right\rangle\left\langle n_{a b}^{\perp}(\hat{z}), s_{d}(z)\right\rangle \\
& =\left\langle\hat{s}_{a}(\hat{z}),-s_{c}(z)+s_{d}(z)\right\rangle\left\langle-\hat{s}_{a}(\hat{z})+\hat{s}_{b}(\hat{z}), s_{d}(z)\right\rangle \\
& =\left\langle\hat{s}_{b}(\hat{z}), s_{d}(z)\right\rangle\left\langle\hat{s}_{a}(\hat{z}), s_{c}(z)\right\rangle^{-1}
\end{aligned}
$$

which proves the lemma.

We now turn to the definition of topological triples. Let $P \rightarrow E \rightarrow B$ be a pair and let $\widehat{P} \rightarrow \widehat{E} \rightarrow B$ be a dual pair with same underlying Hilbert space $\mathcal{H}$. We consider the following diagram of Cartesian squares

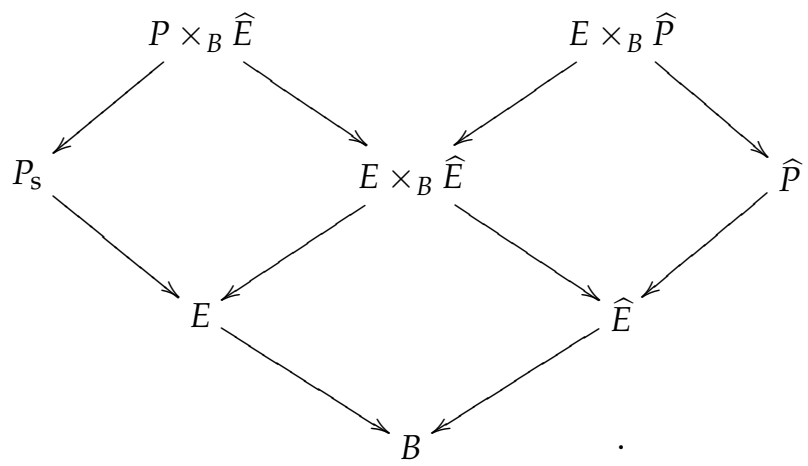

Assume that there is a PU(H)-bundle isomorphism $\kappa: E \times_{B} \widehat{P} \rightarrow P \times_{B} \widehat{E}$ which fits into the above diagram (12), i.e. it fixes its base $E \times_{B} \widehat{E}$. Let us choose a chart $U_{i} \subset B$ common for the pair and the dual pair and trivialise (12) locally. For each $u \in U_{i}$ this induces an automorphism $\kappa_{i}(u)$ of the trivial $\mathrm{PU}(\mathcal{H})$-bundle over $G / N \times \widehat{G} / N^{\perp}$,

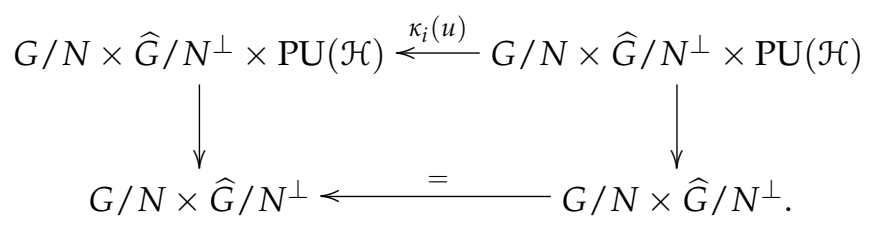

This automorphism defines a Čech class $\left[\kappa_{i}(u)\right] \in \check{H}^{1}\left(G / N \times \widehat{G} / N^{\perp}, \mathrm{U}(1)\right)$ (cp. Therem A.1).

Definition 2.8 We say $\kappa$ satisfies the Poincaré condition if for each chart $U_{i}$ and each $u \in U_{i}$ the equality $\left[\kappa_{i}(u)\right]=\pi+p_{1}^{*} a+p_{2}^{*} b$ holds, for the Poincaré class $\pi$ and some classes $a \in \breve{H}^{1}(G / N, \mathrm{U}(1))$ and $b \in \breve{H}^{1}\left(\widehat{G} / N^{\perp}, \mathrm{U}(1)\right)$. Here $p_{1}, p_{2}$ are the projections from $G / N \times \widehat{G} / \overline{N^{\perp}}$ on the first and second factor. 
Note that in this definition the classes $a, b$ are just of minor importance. They are manifestations of the freedom to choose another atlas as they vary under the change of the local trivialisations. In fact, one can always modify the local trivialisations of the given atlas such that $a$ and $b$ vanish.

Definition 2.9 A topological triple $(\kappa,(P, E),(\widehat{P}, \widehat{E}))$ over $B$ is a pair $(P, E)$ and dual pair $(\widehat{P}, \widehat{E})$ over $B$ (with same underlying Hilbert space $\mathcal{H}$ ) together with a commutative diagram

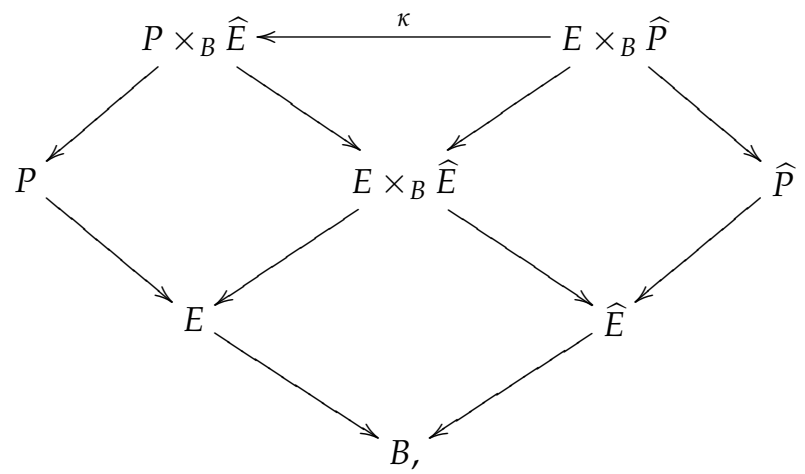

wherein all squares are Cartesian and $\kappa$ is an isomorphism that satisfies the Poincare condition.

We call two topological triples $(\kappa,(P, E),(\widehat{P}, \widehat{E}))$ and $\left(\kappa^{\prime},\left(P^{\prime}, E^{\prime}\right),\left(\widehat{P}^{\prime}, \widehat{E}^{\prime}\right)\right)$ (with underlying Hilbert spaces $\mathcal{H}, \mathcal{H}^{\prime}$ respectively) equivalent if there is a morphisms of pairs $(\varphi, \vartheta, \theta)$ from $(P, E)$ to $\left(P^{\prime}, E^{\prime}\right)$ and a morphism of dual pairs $(\hat{\varphi}, \hat{\vartheta}, \hat{\theta})$ from $(\widehat{P}, \widehat{E})$ to $\left(\widehat{P}^{\prime}, \widehat{E}^{\prime}\right)$ such that the induced diagram

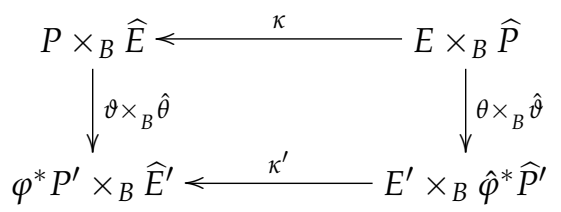

is commutative up to homotopy, i.e. the Čech class of the bundle automorphism $\left(\theta \times_{B} \hat{\vartheta}\right)^{-1} \circ \kappa^{\prime-1} \circ\left(\vartheta \times_{B} \hat{\theta}\right) \circ \kappa$ in $\check{H}^{1}\left(E \times_{B} \widehat{P}, \mathrm{U}(1)\right)$ vanishes. The triples are called stably equivalent if the stabilised triples $\left(\mathbb{1} \otimes \kappa,\left(P_{\mathcal{F}_{1}}, E\right),\left(\widehat{P}_{\mathcal{F}_{1}}, \widehat{E}\right)\right)$ and $\left(\mathbb{1} \otimes \kappa^{\prime},\left(P_{\mathcal{H}_{1}}^{\prime}, E^{\prime}\right),\left(\widehat{P}_{\mathcal{H}_{1}}^{\prime}, \widehat{E}^{\prime}\right)\right)$ are equivalent for some separable Hilbert space $\mathcal{H}_{1}$. The meaning of the index $\mathcal{H}_{1}$ is stabilisation as in equation (4). Stable equivalence will be the right choice of equivalence for us, and we introduce the set valued functor Top which associates to a base space $B$ the set of stable equivalence classes of topological triples, i.e.

$$
\operatorname{Top}(B):=\{\text { topological triples over } B\} / \text { stable equivalence } .
$$


If we choose a $G$ / $N$-bundle $E \rightarrow B$ we can consider all the topological triples with this bundle fixed and those stable equivalences for which the identity over $E$ can be extendend to a morphisms of the underlying pairs. We define

$$
\operatorname{Top}(E, B):=\{\text { topological triples with fixed } E \rightarrow B\} /{\text { stable equivalence over } \text { id }_{E}} .
$$

The automorphisms $\operatorname{Aut}_{B}(E)$ of $E$ over the identity of $B$ act on $\operatorname{Top}(E, B)$ by pullback, and there is a correspondence $\operatorname{Top}(B) \cong \coprod_{[E]}\left(\operatorname{Top}(E, B) / \operatorname{Aut}_{B}(E)\right)$.

Remark 2.4 We already stated in Remark 1.1 that our notion of topological triples in the case of $G=\mathbb{R}^{n}, N=\mathbb{Z}^{n}$ and the notion of T-duality triples as found in [BRS] do not agree. Nevertheless the two notions lead to the same isomorphism classes, but we postpone this clarification to section 3.3, Lemma 3.7.

By definition, the Poincaré class $\pi$ has a geometric interpretation in terms of the Poincaré bundle (11). For our purposes it will be important that we can give an analytical description of $\pi$.

Lemma 2.3 Choose a Borel section $\sigma: G / N \rightarrow G$ and an arbitrary section $\hat{\sigma}$ : $\widehat{G} / N^{\perp} \rightarrow \widehat{G}$ of the corresponding quotient maps. Then

i) the map 9

$$
\begin{aligned}
\kappa^{\sigma}: G / N \times \widehat{G} / N^{\perp} & \rightarrow \operatorname{PU}\left(L^{2}(G / N) \otimes \mathcal{H}\right) \\
(z, \hat{z}) & \mapsto \operatorname{Ad}(\underbrace{\langle\hat{z}), \sigma(-z)-\sigma(-)\rangle}_{=: \bar{\kappa}^{\sigma}(z, \hat{z}) \in L^{\infty}(G / N, \mathrm{U}(1))} \otimes \mathbb{1}_{\mathcal{H}})
\end{aligned}
$$

is continuous and independent of the choice of $\hat{\sigma}$. Therefore it defines (a bundle isomorphism of the trivial $\mathrm{PU}\left(L^{2}(G / N, \mathcal{H})\right)$-bundle and) a class

$$
\left[\kappa^{\sigma}\right] \in \check{H}^{1}\left(G / N \times \widehat{G} / N^{\perp}, \underline{\mathrm{U}(1)}\right),
$$

and this class is independent of the choice of $\sigma$;

ii) $\left[\kappa^{\sigma}\right]=\pi$.

Proof : i) Firstly, we observe that $\widehat{G} \ni \chi \mapsto\langle\chi, \sigma(-)\rangle \in \mathrm{U}\left(L^{2}(G / N)\right)$ is (strongly) continuous. In fact, the sequential continuity of this map ${ }^{10}$ follows by dominated convergence. Therefore the composition

$$
\begin{aligned}
(z, \chi) \mapsto & \lambda_{G / N}(z) \circ\langle\chi, \sigma(-)\rangle \circ \lambda_{G / N}(-z) \circ\langle\chi,-\sigma(-)\rangle \\
& =\langle\chi, \sigma(--z)-\sigma(-)\rangle
\end{aligned}
$$

is continous. Here $\lambda_{G / N}$ is the left regular representation, i.e. $\lambda_{G / N}(z) F(x):=$ $F(-z+x), F \in L^{2}(G / N)$. Now, if $n^{\perp} \in N^{\perp}$ we obtain $\left\langle n^{\perp}, \sigma(--z)-\sigma(-)\right\rangle=$

\footnotetext{
${ }^{9}$ An element $f=f(-) \in L^{\infty}(G / N, \mathrm{U}(1))$ is a multiplication operator on the Hilbert space $L^{2}(G / N)$, see section A.3.

${ }^{10} \widehat{G}$ is first countable.
} 
$\left\langle n^{\perp}, \sigma(-z)\right\rangle \in \mathrm{U}(1) \subset \mathrm{U}\left(L^{2}(G / N)\right.$, because the difference $\sigma(-z)-(\sigma(--z)-$ $\sigma(-))$ is in $N$. Thus $(z, \chi) \mapsto \operatorname{Ad}(\langle\chi, \sigma(--z)-\sigma(-)\rangle)$ factors through the quotient map $G / N \times \widehat{G} \rightarrow G / N \times \widehat{G} / N^{\perp}$ which establishes $\kappa^{\sigma}$ as stated above. To see that the class of $\kappa^{\sigma}$ is independent of $\sigma$ it is sufficient to recognise that $\kappa^{n}$, defined by the same formula as $\kappa^{\sigma}$, is unitary implemented for any Borel function $n: G / N \rightarrow N$. But this is the case, for $(z, \chi) \mapsto\langle\chi, n(--z)-n(-)\rangle \in$ $\mathrm{U}\left(L^{2}(G / N)\right)$ is continuous and factors through the quotient $G / N \times \widehat{G} / N^{\perp}$.

ii) Let $\widehat{G} / N^{\perp} \supset V_{a} \stackrel{\hat{s}_{a}}{\rightarrow} \widehat{G}$ be a family of local sections, so $\hat{s}_{b}(\hat{z})-\hat{s}_{a}(\hat{z})=$ : $n_{a b}^{\perp}(\hat{z}) \in N^{\perp}$ defines a set of transition functions. Let $W_{a}:=G / N \times V_{a}$, then $\kappa_{a}: W_{a} \ni(z, \hat{z}) \mapsto\left\langle\hat{s}_{a}(\hat{z}), \sigma(--z)-\sigma(-)\right\rangle \in \mathrm{U}\left(L^{2}(G / N)\right)$ defines locally a continuous unitary lift of $\kappa^{\sigma}$. Therefore, on twofold intersections $W_{b a} \ni(z, \hat{z})$ we have $\kappa_{b}(z, \hat{z}) \kappa_{a}(z, \hat{z})^{-1}=: \kappa_{a b}(z, \hat{z}) \in \mathrm{U}(1)$, and the class of $\kappa^{\sigma}$ is by definition the class $\left[\kappa_{\text {.. }}\right.$. of the cocycle $\kappa_{. .}$. We obtain

$$
\begin{aligned}
\kappa_{b}(z, \hat{z}) \kappa_{a}(z, \hat{z})^{-1} & =\left\langle\hat{s}_{b}(\hat{z})-\hat{s}_{a}(\hat{z}), \sigma(--z)-\sigma(-)\right\rangle \\
& =\left\langle n_{a b}^{\perp}(\hat{z}), \sigma(--z)-\sigma(-)\right\rangle \\
& =\left\langle n_{a b}^{\perp}(\hat{z}), \sigma(-z)\right\rangle \\
& =\left\langle n_{a b}^{\perp}(\hat{z}), z\right\rangle^{-1} \in \mathrm{U}(1)
\end{aligned}
$$

wherein the last equality identifies $N^{\perp}$ with $\widehat{G / N}$. Therefore the class of $\kappa^{\sigma}$ coincides with the negative of the class of the bundle $Q$.

Of course, the situation is symmetric and there is an analogous statement involving the class of bundle $R$. In that case, if we replace everything by its dual counterpart, we deal with the function

$$
\begin{aligned}
\hat{\kappa}^{\hat{\sigma}}: G / N \times \widehat{G} / N^{\perp} & \rightarrow \operatorname{PU}\left(L^{2}\left(\widehat{G} / N^{\perp}\right) \otimes \mathcal{H}\right) \\
(z, \hat{z}) & \mapsto \operatorname{Ad}(\underbrace{\left\langle\mathbb{1}_{\mathcal{H}}\right)}_{=: \overline{\hat{\kappa}}^{\hat{\sigma}}(z, \hat{z}(. .-\hat{z})-\hat{\sigma}(. .), \sigma(z)\rangle}
\end{aligned}
$$

with a Borel section $\hat{\sigma}: \widehat{G} / N^{\perp} \rightarrow \widehat{G}$.

Lemma 2.4 The class of $\hat{\kappa}^{\hat{\sigma}}$ is the negative of the Poincaré class, i.e. $\pi=\left[\kappa^{\sigma}\right]=$ $-\left[\hat{\kappa}^{\hat{\sigma}}\right]$.

Proof: We show that $(z, \hat{z}) \mapsto \kappa^{\sigma}(z, \hat{z}) \otimes \hat{\kappa}^{\hat{\sigma}}(z, \hat{z})$ is already unitarily imple- 
mented. Indeed,

$$
\begin{aligned}
& \kappa^{\sigma}(z, \hat{z}) \otimes \hat{\kappa}^{\hat{\sigma}}(z, \hat{z}) \\
= & \operatorname{Ad}(\langle\hat{\sigma}(\hat{z}), \sigma(--z)-\sigma(-)\rangle\langle\hat{\sigma}(. .-\hat{z})-\hat{\sigma}(. .), \sigma(z)\rangle) \\
= & \operatorname{Ad}(\langle\hat{\sigma}(\hat{z}), \sigma(--z)-\sigma(-)+\sigma(z)\rangle\langle\hat{\sigma}(. .-\hat{z})-\hat{\sigma}(.), \sigma(z)\rangle) \\
= & \operatorname{Ad}(\langle-\hat{\sigma}(. .-\hat{z})+\hat{\sigma}(. .), \sigma(--z)-\sigma(-)+\sigma(z)\rangle\langle\hat{\sigma}(. .-\hat{z})-\hat{\sigma}(. .), \sigma(z)\rangle) \\
= & \operatorname{Ad}(\langle-\hat{\sigma}(. .-\hat{z})+\hat{\sigma}(. .), \sigma(--z)-\sigma(-)\rangle) \\
= & \left.\operatorname{Ad}\left(\lambda_{\widehat{G} / N^{\perp}}(\hat{z}) \lambda_{G / N}(z)\langle-\hat{\sigma}(. .), \sigma(-)\rangle\right) \lambda_{\widehat{G} / N^{\perp}}(-\hat{z})\langle\hat{\sigma}(. .), \sigma(-)\rangle\right) \lambda_{G / N}(-z) \\
& \left.\left.\quad \lambda_{\widehat{G} / N^{\perp}}(\hat{z})\langle-\hat{\sigma}(. .),-\sigma(-)\rangle\right) \lambda_{\widehat{G} / N^{\perp}}(-\hat{z})\langle\hat{\sigma}(. .),-\sigma(-)\rangle\right) \\
& \in \operatorname{PU}\left(L^{2}(G / N, \mathcal{H}) \otimes L^{2}\left(\widehat{G} / N^{\perp}, \mathcal{H}\right)\right) .
\end{aligned}
$$

The argument of Ad is a continuous, unitary expression, since the left regular representations $\lambda_{\widehat{G} / N^{\perp}}$ and $\lambda_{G / N}$ are (strongly) continuous. 


\section{T-Duality}

In the last sections we have introduced our main objects (dynamical and topological triples). In the following sections we single out specific subclasses of those and show that they are related to each other. In addition, we show that their relations are precisely those which are obtained from the associated $C^{*}$ dynamical picture. Most part of this $C^{*}$-algebraic structure been observed in context of continuous trace algebras alone in [RR, Thm 2.2, Cor. 2.5], [OR, Cor. 2.1] and more recent in [MR, Thm. 3.1] with applications to T-duality.

However, we establish a different approach by use of the local structure of the underlying objects. In particular, we do not need any assumption about local compactness of the underlying spaces. If we assume our base spaces $B$ to be locally compact, then the bundles $E, \widehat{E}$ over $B$ will be locally compact and spectra of the involved continuous trace algebras; but the proof we present is independent of such an assumption. Moreover, it has the advantage of being explicit enough to point out the connexion between the $C^{*}$-algebraic approach and the topological approach to T-duality.

Notation: In several proofs of the following sections we have to check the validity of local identities. All of these are straight forward computations in general, but to keep the formulas readable we drop the base variable $u \in B$. E.g. an identity like

$$
\zeta_{j i}(u)(z+g N) \mu_{i}(u)(g, z)=\mu_{j}(u)\left(g, g_{j i}(u)+z\right) \zeta_{j i}(u)(z)
$$

becomes

$$
\zeta_{j i}(z+g N) \mu_{i}(g, z)=\mu_{j}\left(g, g_{j i}+z\right) \zeta_{j i}(z) .
$$

We indicate this with the label ${ }^{(1)}$ before any of these computations.

\subsection{The Duality Theory of Dynamical Triples}

We start with the definition of an important subclass of dynamical triples.

Definition 3.1 A dynamical triple is called dualisable if its associated total cohomology class (Proposition 2.8) is of the form $\left[\psi_{\ldots} . ., \phi_{. .}, \omega .=d_{*} v\right.$. $]$, i.e. the pair permits a sufficiently refined atlas such that $\omega$. is in the image of the boundary operator $d_{*}$.

Let us denote the set of dualisable dynamical triples over $B$ by $\operatorname{Dyn}^{\dagger}(B)$, respectively $\operatorname{Dyn}^{\dagger}(E, B)$ for fixed $E$. Similarly, $\widehat{\operatorname{Dyn}}^{\dagger}(B)$ and $\widehat{\operatorname{Dyn}}^{\dagger}(E, B)$ are the sets of dualisable dual dynamical triples. We are going to present a construction that associates to a dualisable dynamical triple $x$ a dual dualisable dynamical triple $\widehat{x}$. This construction will be an honest map on the level of equivalence classes, and there it turns out to be involutive, i.e. $[\widehat{x}]=[x]$.

Let $(\rho, P, E)$ be a dualisable dynamical triple over $B$. Choose a sufficiently refined atlas $\left\{U_{i} \mid i \in I\right\}$ in the sense that the transition functions $\zeta_{i j}$ and cocycles 
$\mu_{i}$ lift continuously to $\bar{\zeta}_{i j}$ and $\bar{\mu}_{i}$. Then let $\left(\psi_{\ldots, .}, \phi_{. .}, \omega\right)$ be the associated 2cocycle. Since the triple is dualisable, we can assume that $\omega$. is in the image of $d_{*}$, let $\omega .:=d_{*} v_{\text {. }}$. We can modify $\bar{\mu}_{i}$ by multiplying with $v_{i}^{-1}$. Therefore we can assume without restriction that $d\left(\bar{\mu}_{i}(u)\right)=\mathbb{1}$ and $\omega_{i}=1$, for all $i \in I, u \in U_{i}$. $\bar{\mu}_{i}$ is then unique up to a function $U_{i} \rightarrow Z_{\mathrm{Bor}}^{1}\left(G, L^{\infty}(G / N, \mathrm{U}(1))\right)$.

We are going to define a set of transition functions

$$
\hat{a}_{i j}:=\hat{g}_{i j} \times \hat{\zeta}_{i j}: U_{j i} \rightarrow \widehat{G} / N^{\perp} \ltimes \operatorname{Map}\left(\widehat{G} / N^{\perp}, \mathrm{PU}\left(L^{2}(G / N) \otimes \mathcal{H}\right)\right)
$$

for a dual pair. The vanishing of $\omega$. implies that $\phi_{i j}(u) \in Z_{\text {Bor }}^{1}\left(G, L^{\infty}(G / N ; \mathrm{U}(1))\right)$, for all $i, j, u$. This tells us ${ }^{(i)}$

$$
\begin{aligned}
\phi_{i j}\left(g, h N+_{-}\right) \phi_{i j}\left(h,_{-}\right) & =\phi_{i j}\left(g+h,_{-}\right) \\
& =\phi_{i j}\left(h+g_{r_{-}}\right) \\
& =\phi_{i j}\left(h, g N+_{-}\right) \phi_{i j}\left(g_{r_{-}}\right) \in L^{\infty}(G / N, \mathrm{U}(1))
\end{aligned}
$$

which implies for $g=n \in N$ and all $h N \in G / N$ that $\phi_{k l}(u)\left(n, h N+_{-}\right)=$ $\phi_{k l}(u)\left(n,_{-}\right)$holds, hence $\phi_{k l}(u)(n,-) \in L^{\infty}(G / N, \mathrm{U}(1))$ is a constant. Further, $n \mapsto \phi_{k l}(u)(n,-)$ is a homomorphism and continuous as $N$ is discrete. Thus there exists $\hat{g}_{i j}: U_{j i} \rightarrow \widehat{G} / N^{\perp}(\cong \widehat{N})$ such that

$$
\left\langle\hat{g}_{i j}(u), n\right\rangle=\left(\phi_{i j}(u)(n, z)\right)^{-1}
$$

for all $u \in U_{j i}, n \in N$ and (almost) all $z \in G / N$.

Proposition $3.1 \quad\left\{\hat{g}_{i j}: U_{j i} \rightarrow \widehat{G} / N^{\perp} \mid i, j \in I\right\}$ is a Čech 1-cocycle.

Proof : We have $\delta_{g} \phi=d_{*} \psi$, explicitely this reads for $g \in G, u \in U_{i j k}$

$$
\phi_{j k}(u)\left(g_{-}\right) \phi_{i k}(u)\left(g_{-}\right)^{-1} \phi_{i j}(u)\left(g, g_{j k}(u)+_{-}\right)=\psi_{i j k}(u)\left({ }_{-}+g N\right) \psi_{i j k}(u)\left(_{-}\right)^{-1} .
$$

For $g=n \in N$ the right-hand side vanishes, hence the Čech cocycle equation follows.

In the next lemma we state some properties of the lifted cocycles $\bar{\mu}_{i}$. It will be a useful technical tool later.

Lemma 3.1 The maps

(i) $U_{i} \times G /\left.N \ni(u, z) \mapsto \bar{\mu}_{i}(u)(., z)\right|_{N} \in \mathrm{U}\left(L^{2}(N) \otimes \mathcal{H}\right)$,

(ii) $U_{i} \times G / N \ni(u, z) \mapsto \operatorname{Ad}\left(\bar{\mu}_{i}(u)(., z)\right) \in \operatorname{PU}\left(L^{2}(G) \otimes \mathcal{H}\right)$,

(iii) $U_{i} \times G / N \ni(u, z) \mapsto \operatorname{Ad}\left(\bar{\mu}_{i}(u)(-\sigma(-), z)\right) \in \operatorname{PU}\left(L^{2}(G / N) \otimes \mathcal{H}\right)$,

(iv) $U_{j i} \times G / N \ni(u, z) \mapsto \operatorname{Ad}\left(\phi_{i j}(u)(., z)\right) \in \operatorname{PU}\left(L^{2}(G)\right)$, 
(v) $U_{j i} \times G / N \ni(u, z) \mapsto \operatorname{Ad}\left(\phi_{i j}(u)(-\sigma(-), z)\right) \in \operatorname{PU}\left(L^{2}(G / N)\right)$

are continuous; and for all $u \in U_{j i}, z \in G / N$ the formula

$$
\begin{aligned}
& \operatorname{Ad}\left(\bar{\zeta}_{j i}(u)\left(-_{-}\right) \phi_{j i}(u)(-\sigma(-), 0)^{-1}\right) \\
= & \operatorname{Ad}\left(\bar{\mu}_{j}(u)\left(-\sigma(-+z), g_{j i}(u)+z\right)\right) \zeta_{j i}(u)(z) \operatorname{Ad}\left(\bar{\mu}_{i}(u)(-\sigma(-+z), z)^{-1}\right) \\
& \operatorname{Ad}\left(\left\langle\hat{\sigma}\left(\hat{g}_{j i}(u)\right), \sigma(-+z)-\sigma(-)\right\rangle\right) \\
& \in \operatorname{PU}\left(L^{2}(G / N, \mathcal{H})\right)
\end{aligned}
$$

holds.

Proof : (i) Let $n \in N, g \in G$ and $z \in G / N$, then

$$
\begin{aligned}
\bar{\mu}_{i}(u)(n, z+g N) \bar{\mu}_{i}(u)(g, z) & =\bar{\mu}_{i}(u)(n+g, z) \\
& =\bar{\mu}_{i}(u)(g, z) \bar{\mu}_{i}(u)(n, z) .
\end{aligned}
$$

This implies that $\bar{\mu}_{i}(u)(n, z+g N)=\mu_{i}(u)(g, z)\left(\bar{\mu}_{i}(u)(n, z)\right) \in \mathrm{U}(\mathcal{H})$. Therein the right hand side is continuous in $g$, hence the left hand side is continuous in $z^{\prime}:=g N$. As the left hand side is symmetric in $z$ and $z^{\prime}$ it is continuos in $z$.

(ii) Let $\left(u_{\alpha}, z_{\alpha}\right) \rightarrow(u, z)$ be a converging net. Let $x_{\alpha}:=z_{\alpha}-z$ and choose $g_{\alpha} \rightarrow 0 \in G$ such that $g_{\alpha} N=x_{\alpha}$. Such $g_{\alpha}$ exist - take a local section of the quotient (Lemma A.1). Then

$$
\begin{aligned}
\operatorname{Ad}\left(\bar{\mu}_{i}\left(u_{\alpha}\right)\left(., z_{\alpha}\right)\right) & =\operatorname{Ad}\left(\bar{\mu}_{i}\left(u_{\alpha}\right)\left(., z+x_{\alpha}\right)\right) \\
& =\operatorname{Ad}\left(\bar{\mu}_{i}\left(u_{\alpha}\right)\left(.+g_{\alpha}, z\right)\right) \operatorname{Ad}\left(\bar{\mu}_{i}\left(u_{\alpha}\right)\left(g_{\alpha}, z\right)^{-1}\right) \\
& =\operatorname{Ad}\left(\bar{\mu}_{i}\left(u_{\alpha}\right)\left(.+g_{\alpha}, z\right)\right) \mu_{i}\left(u_{\alpha}\right)\left(g_{\alpha}, z\right)^{-1}
\end{aligned}
$$

The second factor converges to $\mu_{i}(u)(0, z)=\mathbb{1}$. For the first factor, note that $(u, g) \mapsto \operatorname{Ad}\left(\bar{\mu}_{i}(u)(.+g, z)\right)=\operatorname{Ad}\left(\lambda_{G}(g) \bar{\mu}_{i}(u)(., z) \lambda_{G}(-g)\right) \in \operatorname{PU}\left(L^{2}(G, \mathcal{H})\right)$ is continuous. This because $\operatorname{Bor}(G \times G / N, \mathrm{U}(\mathcal{H})) \ni \bar{\mu}_{i}(u) \mapsto \bar{\mu}_{i}(u)(., z) \in$ $L^{\infty}(G, \mathrm{U}(\mathcal{H}))$ is continuous.

(iii) We shall show that $v(.) \mapsto v\left(\sigma\left({ }_{-}\right)\right)$is a continuous map from the multiplication operators $L^{\infty}(G, \mathrm{U}(\mathcal{H}))$ to $L^{\infty}(G / N, \mathrm{U}(\mathcal{H}))$. Indeed, let $f \in L^{2}(G / N)$ and let $\chi$ be the characteristic function of $\sigma(G / N) \subset G$, so $\left(g \mapsto f_{\sigma}(g):=\right.$ $f(g N) \chi(g)) \in L^{2}(G)$. Let $v_{n}(.) \rightarrow v(.) \in L^{\infty}(G, \mathrm{U}(\mathcal{H}))$ be a converging sequence. Then

$$
\begin{aligned}
\| v_{n}\left(\sigma\left({ }_{-}\right)\right) f\left(\left(_{-}\right)-v(\sigma(-)) f(-) \|^{2}\right. & =\int_{G / N}\left|v_{n}(\sigma(z)) f(z)-v(\sigma(z)) f(z)\right|^{2} d z \\
& =\int_{G}\left|v_{n}(g) f_{\sigma}(g)-v(g) f_{\sigma}(g)\right|^{2} d g \\
& \rightarrow 0, \text { for } n \rightarrow \infty .
\end{aligned}
$$

$(i v),(v)$ The fourth and fifth statement follow directly from (i) and (ii) and from the definition of $\phi_{j i}$ on page 30 . 
Equation (17) follows by threefold application of the definition of $\phi_{j i}$. We let $n(x, y):=\sigma(x+y)-\sigma(x)-\sigma(y) \in N$, then ${ }^{\circledR}$

$$
\begin{aligned}
& \operatorname{Ad}\left(\bar{\zeta}_{j i}\left(-{ }_{-}\right) \phi_{j i}(-\sigma(-), 0)^{-1}\right) \\
= & \operatorname{Ad}\left(\bar{\mu}_{j}\left(-\sigma(-), g_{j i}\right) \bar{\zeta}_{j i}(0) \bar{\mu}_{i}(-\sigma(-), 0)^{-1}\right) \\
= & \operatorname{Ad}\left(\bar{\mu}_{j}\left(-\sigma(-), g_{j i}\right) \bar{\mu}_{j}\left(\sigma(z), g_{j i}\right)^{-1} \bar{\zeta}_{j i}(z)\right. \\
& \left.\bar{\mu}_{i}(\sigma(z), 0) \bar{\mu}_{i}\left(-\sigma\left({ }_{-}\right), 0\right)^{-1}\right) \\
= & \operatorname{Ad}\left(\bar{\mu}_{j}\left(-\sigma(-)-\sigma(z), g_{j i}+z\right) \bar{\zeta}_{j i}(z)\right. \\
& \left.\bar{\mu}_{i}(-\sigma(-)-\sigma(z), z)^{-1}\right) \\
= & \operatorname{Ad}\left(\bar{\mu}_{j}\left(-\sigma(-+z), g_{j i}+z\right) \bar{\mu}_{j}\left(n(-, z), g_{j i}+z\right) \bar{\zeta}_{j i}(z)\right. \\
& \left.\bar{\mu}_{i}(n(-, z), z)^{-1} \bar{\mu}_{i}(-\sigma(-+z), z)^{-1}\right) \\
= & \operatorname{Ad}\left(\bar{\mu}_{j}\left(-\sigma(-+z), g_{j i}+z\right) \bar{\zeta}_{j i}(z)\right. \\
& \left.\phi_{j i}\left(n\left({ }_{-}, z\right), z\right)^{-1} \bar{\mu}_{i}(-\sigma(-+z), z)^{-1}\right) .
\end{aligned}
$$

Since $\operatorname{Ad}\left(\phi_{j i}(u)\left(n\left({ }_{-}, z\right), z\right)^{-1}\right)=\operatorname{Ad}\left(\left\langle\hat{g}_{j i}(u), n\left({ }_{-}, z\right)\right\rangle\right)=\operatorname{Ad}\left(\left\langle\hat{\sigma}\left(g_{j i}(u)\right), \sigma\left(_{-}+\right.\right.\right.$ $z)-\sigma(-)\rangle)$, the assertion is proven.

We now turn to the definition of the projective unitary transition functions $\hat{\zeta}_{j i}$ for the dual pair, but before we remark on the local definition we make.

Remark 3.1 The ad hoc definition of the transition functions of the dual pair by formula (18) below may seem very unsatisfactory, because one cannot even guess the origin of this formula. In Theorem 3.8 we will see that the crossed product $G \times{ }_{\rho} \Gamma(E, F)$ (see appendix A.4) of the associated $C^{*}$-algebra of sections $\Gamma(E, F), F:=P \times_{\mathrm{PU}(\mathcal{H})}$ $\mathcal{K}(\mathcal{H})$, can be explicitly computed by a fibre-wise, modified Fourier transform. The behaviour of this transformation under the change of charts will lead finally to formula (18) (and shows that the crossed product is isomorphic to the associated $C^{*}$-algebra of sections of the dual pair).

However, we are in the pleasant situation that we can avoid the $C^{*}$-algebraic apparatus at this point and can formulate the theory in bundle theoretic terms only.

By Lemma 3.1, $(u, z) \mapsto \operatorname{Ad}\left(\phi_{j i}(u)(-\sigma(-), z)\right)$ is continuous hence its restriction to $U_{j i} \times\{0\}$ is: $u \mapsto \operatorname{Ad}\left(\phi_{j i}(u)(-\sigma(-), 0)\right)$. Then we let

$$
\begin{gathered}
\hat{\zeta}_{j i}(u)(\hat{z}):=\operatorname{Ad}\left(( \overline { \kappa } ^ { \sigma } ( - g _ { j i } ( u ) , \hat { g } _ { j i } ( u ) + \hat { z } ) \otimes \mathbb { 1 } _ { \mathcal { H } } ) \left(\lambda_{G / N}\left(-g_{j i}(u) \otimes \mathbb{1}_{\mathcal{H}}\right)\right.\right. \\
\left.\bar{\zeta}_{j i}(u)\left(-_{-}\right)\left(\phi_{j i}(u)(-\sigma(-), 0)^{-1} \otimes \mathbb{1}_{\mathcal{H}}\right)\right) .
\end{gathered}
$$


Therein $\lambda_{G / N}$ is the left regular representation on $L^{2}(G / N)$, and $\bar{\kappa}^{\sigma}$ is taken from Lemma 2.3. Indeed, this defines a continuous map

$$
\hat{\zeta}_{j i}: U_{j i} \rightarrow \operatorname{Map}\left(\widehat{G} / N^{\perp}, \operatorname{PU}\left(L^{2}(G / N) \otimes \mathcal{H}\right)\right),
$$

but its definition involved several choices, namely, the atlas, the liftings $\bar{\zeta}_{i j}, \bar{\mu}_{i}$ and the section $\sigma$.

Theorem 3.1 The family $\hat{a}_{. .}=\left\{\hat{a}_{i j}=g_{j i} \times \zeta_{j i} \mid i, j \in I\right\}$ is a Čech cocycle and its class $\left[\hat{a}_{. .}\right]$is independent of the choices involved.

Proof : We have to show that $\delta_{\hat{g}} \hat{\zeta}=\mathbb{1}$. We insert (18) and obtain ${ }^{(1)}$

$$
\begin{aligned}
& \hat{\zeta}_{j i}(\hat{z}) \hat{\zeta}_{k i}(\hat{z})^{-1} \hat{\zeta}_{k j}\left(\hat{g}_{j i}+\hat{z}\right) \\
= & \operatorname{Ad}\left(\left(\left\langle\hat{\sigma}\left(\hat{g}_{j i}+\hat{z}\right), \sigma\left({ }_{-}+g_{j i}\right)-\sigma(-)\right\rangle \otimes \mathbb{1}\right)\right. \\
& \left(\lambda_{G / N}\left(-g_{j i}\right) \otimes \mathbb{1}\right) \bar{\zeta}_{j i}\left(-_{-}\right)\left(\phi_{j i}\left(-\sigma\left(_{-}\right), 0\right)^{-1} \otimes \mathbb{1}\right) \\
& \left(\phi_{k i}(-\sigma(-), 0) \otimes \mathbb{1}\right) \bar{\zeta}_{k i}\left(-_{-}\right)^{-1}\left(\lambda_{G / N}\left(g_{k i}\right) \otimes \mathbb{1}\right) \\
& \left(\left\langle\hat{\sigma}\left(\hat{g}_{k i}+\hat{z}\right), \sigma\left(-+g_{k i}\right)-\sigma(-)\right\rangle^{-1} \otimes \mathbb{1}\right) \\
& \left(\left\langle\hat{\sigma}\left(\hat{g}_{k j}+\hat{g}_{j i}+\hat{z}\right), \sigma\left(-+g_{k j}\right)-\sigma(-)\right\rangle \otimes \mathbb{1}\right) \\
& \left.\left(\lambda_{G / N}\left(-g_{k j}\right) \otimes \mathbb{1}\right) \bar{\zeta}_{k j}\left(--_{-}\right)\left(\phi_{k j}(-\sigma(-), 0)^{-1} \otimes \mathbb{1}\right)\right) \\
= & \operatorname{Ad}\left(\left(\left\langle\hat{\sigma}\left(\hat{g}_{j i}+\hat{z}\right)-\hat{\sigma}\left(\hat{g}_{k i}+\hat{z}\right), \sigma\left(-+g_{j i}\right)-\sigma(-)\right\rangle \otimes \mathbb{1}\right)\right. \\
& \left(\psi_{k j i}\left(-g_{-}-g_{j i}\right) \otimes \mathbb{1}\right)\left(\phi_{j i}\left(-\sigma\left(-+g_{j i}\right), 0\right)^{-1} \otimes \mathbb{1}\right) \\
& \left.\left(\phi_{k i}\left(-\sigma\left(-+g_{j i}\right), 0\right) \otimes \mathbb{1}\right)\left(\phi_{k j}(-\sigma(-), 0)^{-1} \otimes \mathbb{1}\right)\right) .
\end{aligned}
$$

The argument of Ad has simplified to a multiplication operator, so it remains to show that it is a constant in $\mathrm{U}(1)$. By use of $\delta_{g} \phi=d_{*} \psi$ and $d_{*} \phi=1$ we continue

$$
\begin{aligned}
\ldots= & \operatorname{Ad}\left(\left(\left\langle\hat{\sigma}\left(\hat{g}_{j i}+\hat{z}\right)-\hat{\sigma}\left(\hat{g}_{k i}+\hat{z}\right), \sigma\left({ }_{-}+g_{j i}\right)-\sigma(-)\right\rangle \otimes \mathbb{1}\right)\right. \\
& \left.\psi_{k j i}(0)\left(\phi_{k j}\left(-\sigma\left(-+g_{j i}\right), g_{j i}\right) \otimes \mathbb{1}\right)\left(\phi_{k j}\left(-\sigma\left(_{-}\right), 0\right)^{-1} \otimes \mathbb{1}\right)\right) \\
= & \operatorname{Ad}\left(\left(\left\langle\hat{\sigma}\left(\hat{g}_{j i}+\hat{z}\right)-\hat{\sigma}\left(\hat{g}_{k i}+\hat{z}\right), \sigma\left(-+g_{j i}\right)-\sigma(-)\right\rangle \otimes \mathbb{1}\right)\right. \\
& \left.\psi_{k j i}(0)\left(\phi_{k j}\left(-\sigma(-)+\sigma\left(-+g_{j i}\right), 0\right)^{-1} \otimes \mathbb{1}\right)\right) \\
= & \operatorname{Ad}\left(\psi_{k j i}(0)\left(\phi_{k j}\left(-\sigma(-)+\sigma\left(-+g_{j i}\right), 0\right)^{-1} \otimes \mathbb{1}\right)\right. \\
& \left\langle\hat{\sigma}\left(\hat{g}_{j i}+\hat{z}\right)-\hat{\sigma}\left(\hat{g}_{k i}+\hat{z}\right), \sigma\left(g_{j i}\right)\right\rangle \\
& \left.\left(\langle\hat{\sigma}\left(\hat{g}_{j i}+\hat{z}\right)-\hat{\sigma}\left(\hat{g}_{k i}+\hat{z}\right), \underbrace{\sigma\left(-+g_{j i}\right)-\sigma(-)-\sigma\left(g_{j i}\right)}_{\in N}\rangle \otimes \mathbb{1}\right)\right),
\end{aligned}
$$


this can be transformed by use of the definition of $\hat{g}_{j i}$

$$
\begin{aligned}
\ldots= & \operatorname{Ad}\left(\psi_{k j i}(0)\left(\phi_{k j}\left(-\sigma(-)+\sigma\left(-+g_{j i}\right), 0\right)^{-1} \otimes \mathbb{1}\right)\right. \\
& \left\langle\hat{\sigma}\left(\hat{g}_{j i}+\hat{z}\right)-\hat{\sigma}\left(\hat{g}_{k i}+\hat{z}\right), \sigma\left(g_{j i}\right)\right\rangle \\
& \left.\left(\left\langle-\hat{g}_{k j}, \sigma\left(-+g_{j i}\right)-\sigma(-)-\sigma\left(g_{j i}\right)\right\rangle \otimes \mathbb{1}\right)\right) \\
= & \operatorname{Ad}\left(\psi_{k j i}(0) \phi_{k j}\left(\sigma\left(g_{j i}\right), 0\right)^{-1}\left\langle\hat{\sigma}\left(\hat{g}_{j i}+\hat{z}\right)-\hat{\sigma}\left(\hat{g}_{k i}+\hat{z}\right), \sigma\left(g_{j i}\right)\right\rangle\right) \\
= & \mathbb{1}_{L^{2}(G / N) \otimes \mathcal{H} .}
\end{aligned}
$$

This is the desired result.

To check that all the choices involved have no effect on the class of this cocycle is straight forward, and we skip the tedious computation here.

Let us define a pair for $\hat{g}_{i j}, \hat{\zeta}_{i j}$ explicitly. Let

$$
\widehat{E}:=\coprod_{i}\left(U_{i} \times \widehat{G} / N^{\perp}\right) / \sim
$$

with relation $(i, u, \hat{z}) \sim\left(j, u, \hat{g}_{j i}(u)+\hat{z}\right)$ and

$$
\widehat{P}:=\coprod_{i}\left(U_{i} \times \widehat{G} / N^{\perp} \times \operatorname{PU}\left(L^{2}(G / N) \otimes \mathcal{H}\right)\right) / \sim
$$

with relation $(i, u, \hat{z}, U) \sim\left(j, u, \hat{g}_{j i}(u)+\hat{z}, \hat{\zeta}_{j i}(u)(\hat{z}) U\right) .(\widehat{P}, \widehat{E})$ is a dual pair over $B$ with underlying Hilbert space $\widehat{\mathcal{H}}:=L^{2}(G / N) \otimes \mathcal{H}$. Then the statement of the theorem is that we have constructed a map

$$
\begin{aligned}
\operatorname{Dyn}^{\dagger}(B) & \rightarrow \widehat{\operatorname{Par}}(B), \\
{[(\rho, P, E)] } & \mapsto[(\widehat{P}, \widehat{E})]
\end{aligned}
$$

for one easily checks that outer conjugate triples define isomorphic duals. We are going to improve this statement in the next theorem.

Let us define a family of projective unitary 1-cocycles by the following simple formula. Let $\chi \in \widehat{G}, \hat{z} \in \widehat{G} / N^{\perp}, u \in U_{i}$, then we define

$$
\hat{\mu}_{i}(u)(\chi, \hat{z}):=\operatorname{Ad}\left(\langle\chi,-\sigma(-)\rangle \otimes \mathbb{1}_{\mathcal{H}}\right) \in \operatorname{PU}\left(L^{2}(G / N) \otimes \mathcal{H}\right) .
$$

Therein $\sigma: G / N \rightarrow G$ is the same Borel section which we have used to define $\hat{\zeta}_{j i}$. By dominated convergence ${ }^{11}$, it is clear that $\chi \mapsto\langle\chi,-\sigma(-)\rangle \in$ $L^{\infty}(G / N, \mathrm{U}(1))$ is continuous, so $\hat{\mu}_{i}$ is.

Theorem 3.2 The family $\left\{\hat{\mu}_{i} \mid i \in I\right\}$ defines a dual decker $\hat{\rho}$ on the pair $(\widehat{P}, \widehat{E})$, and we obtain a bijection from the set of dualisable dynamical triples to the set of dualisable dual dynamical triples,

$$
\begin{aligned}
\operatorname{Dyn}^{\dagger}(B) & \rightarrow \widehat{\operatorname{Dyn}}^{\dagger}(B) \\
{[\rho, P, E] } & \mapsto[\hat{\rho}, \widehat{P}, \widehat{E}]
\end{aligned}
$$

$11 \widehat{G}$ is first countable. 
In formulas (16),(18) and (20) we can replace everything by its dual counterpart, i.e. we interchange the rôle of triples and dual triples, then we obtain a map

$$
\widehat{\operatorname{Dyn}}^{\dagger}(B) \rightarrow \operatorname{Dyn}^{\dagger}(B) \text {. }
$$

Moreover, these two maps are natural and inverse to each other, so we have an equivalence of functors

$$
\mathrm{Dyn}^{\dagger} \cong \widehat{\mathrm{Dyn}}^{+} \text {. }
$$

Proof : To see that the cocycles $\hat{\mu}_{i}$ define a decker we have to verify that

$$
\hat{\mu}_{i}(u)(\chi, \hat{z})=\hat{\zeta}_{j i}(u)\left(\hat{z}+\chi N^{\perp}\right)^{-1} \hat{\mu}_{j}(u)\left(\chi, \hat{g}_{j i}(u)+\hat{z}\right) \hat{\zeta}_{j i}(u)(\hat{z}) .
$$

We just compute the right-hand side ${ }^{\circledR}$

$$
\begin{aligned}
& \hat{\zeta}_{j i}\left(\hat{z}+\chi N^{\perp}\right)^{-1} \hat{\mu}_{j}\left(\chi, \hat{g}_{j i}+\hat{z}\right) \hat{\zeta}_{j i}(\hat{z}) \\
= & \operatorname{Ad}\left(\bar{\zeta}_{j i}\left(-_{-}\right)^{-1}\left(\phi_{j i}(-\sigma(-), 0) \otimes \mathbb{1}\right)\left(\lambda_{G / N}\left(g_{j i}\right) \otimes \mathbb{1}\right)\right. \\
& \left(\left\langle\hat{\sigma}\left(\hat{z}+\chi N^{\perp}+\hat{g}_{j i}\right), \sigma(-)-\sigma\left(-+g_{j i}\right)\right\rangle \otimes \mathbb{1}\right) \\
& (\langle\chi,-\sigma(-)\rangle \otimes \mathbb{1}) \\
& \left(\left\langle\hat{\sigma}\left(\hat{z}+\hat{g}_{j i}\right),-\sigma(-)+\sigma\left(-+g_{j i}\right)\right\rangle \otimes \mathbb{1}\right) \\
& \left.\left(\lambda_{G / N}\left(-g_{j i}\right) \otimes \mathbb{1}\right) \bar{\zeta}_{j i}\left(-_{-}\right)\left(\phi_{j i}(-\sigma(-), 0)^{-1} \otimes \mathbb{1}\right)\right) \\
= & \operatorname{Ad}\left(\left(\left\langle\hat{\sigma}\left(\hat{z}+\chi N^{\perp}+\hat{g}_{j i}\right), \sigma\left(--g_{j i}\right)-\sigma(-)\right\rangle \otimes \mathbb{1}\right)\right. \\
& \left(\left\langle\chi,-\sigma\left(--g_{j i}\right)\right\rangle \otimes \mathbb{1}\right) \\
& \left.\left(\left\langle\hat{\sigma}\left(\hat{z}+\hat{g}_{j i}\right),-\sigma\left(--g_{j i}\right)+\sigma(-)\right\rangle \otimes \mathbb{1}\right)\right) \\
= & \operatorname{Ad}\left(\left(\langle\chi,-\sigma(-)\rangle\left\langle\hat{\sigma}\left(\hat{z}+\hat{g}_{j i}+\chi N^{\perp}\right)-\chi-\hat{\sigma}\left(\hat{z}+\hat{g}_{j i}\right), \sigma\left(--g_{j i}\right)-\sigma(-)\right) \otimes \mathbb{1}\right)\right) \\
= & \operatorname{Ad}((\langle\chi,-\sigma(-)\rangle \otimes \mathbb{1}) \underbrace{\left.\left.\hat{g}_{j i}+\chi N^{\perp}\right)-\chi-\hat{\sigma}\left(\hat{z}+\hat{g}_{j i}\right),-\sigma\left(g_{j i}\right)\right\rangle}_{=:\left(\hat{\sigma}_{j i}(\chi, \hat{z})\right)^{-1} \equiv \mathbb{1} \bmod \mathrm{U}(1)}) \\
= & \hat{\mu}_{i}(\chi, \hat{z}) .
\end{aligned}
$$

This establishes $\hat{\rho}$. A careful look at this calculation shows that $\hat{\phi}$.. indeed is the second term of the total dual cocycle $\left(\hat{\psi}_{. . .}, \hat{\phi}_{. .,}, 1\right)$ defined by our constructed dual dynamical triple. It is easy to see that another choice of the section $\sigma$ alters $\hat{\mu}_{i}$ and $\hat{\zeta}_{j i}$ precisely in such a way that they define an isomorphic pair. So we have established a map $\operatorname{Dyn}^{\dagger}(B) \rightarrow \widehat{\operatorname{Dyn}}^{\dagger}(B)$ and, by replacing everything by its dual, a map in opposite direction.

To prove the remaining assertions of the theorem, we shall apply our construction twice. We will find that the double dual $(\hat{\rho}, \widehat{P}, \widehat{\widehat{E}})$ is isomorphic to $\left(\right.$ Ad $\left.\circ \lambda_{G} \otimes \rho, P U\left(L^{2}(G)\right) \otimes P, E\right)$. In particular, we already see from the definition of $\hat{\phi}_{j i}$ that the double dual bundle $\widehat{E}$ has cocycle $\hat{\delta}_{j i}(u):=\left.\hat{\phi}_{j i}(u)\left(_{-}, 0\right)\right|_{N^{\perp}}=$ 
$g_{j i}(u)$, so there is $\theta: \widehat{\widehat{E}} \cong E$. We compute the double dual cocycle

$$
\hat{\hat{\zeta}}_{j i}: U_{i j} \rightarrow \operatorname{Map}\left(G / N, \operatorname{PU}\left(L^{2}\left(\widehat{G} / N^{\perp}\right) \otimes L^{2}(G / N) \otimes \mathcal{H}\right)\right) .
$$

By definition, we have by (18) and (15) ${ }^{(1)}$

$$
\begin{aligned}
& \hat{\hat{\zeta}}_{j i}(z) \\
= & \operatorname{Ad}\left(\left(\overline{\hat{\kappa}}^{\hat{\sigma}}\left(g_{j i}+z,-\hat{g}_{j i}\right) \otimes \mathbb{1} \otimes \mathbb{1}\right)\left(\lambda_{\hat{G} / N^{\perp}}\left(-\hat{g}_{j i}\right) \otimes \mathbb{1} \otimes \mathbb{1}\right)\right. \\
& \left.\bar{\zeta}_{j i}(-. .)\left(\hat{\phi}_{j i}(-\hat{\sigma}(. .), 0)^{-1} \otimes \mathbb{1} \otimes \mathbb{1}\right)\right) \\
= & \operatorname{Ad}\left(\left(\overline{\hat{\kappa}}^{\hat{\sigma}}\left(g_{j i}+z,-\hat{g}_{j i}\right) \otimes \mathbb{1} \otimes \mathbb{1}\right)\left(\lambda_{\widehat{\hat{G}} / N^{\perp}}\left(-\hat{g}_{j i}\right) \otimes \mathbb{1} \otimes \mathbb{1}\right)\right. \\
& \left(\bar{\kappa}^{\sigma}\left(-g_{j i}, \hat{g}_{j i}-. .\right) \otimes \mathbb{1}\right)\left(\mathbb{1} \otimes \lambda_{G / N}\left(-g_{j i}\right) \otimes \mathbb{1}\right) \\
& \left.\left(\mathbb{1} \otimes \bar{\zeta}_{j i}\left(-{ }_{-}\right)\right)\left(\mathbb{1} \otimes \phi_{j i}(-\sigma(-), 0)^{-1} \otimes \mathbb{1}\right)\left(\hat{\phi}_{j i}(-\hat{\sigma}(. .), 0)^{-1} \otimes \mathbb{1} \otimes \mathbb{1}\right)\right) .
\end{aligned}
$$

With equation (17) and the definitions of $\bar{\kappa}^{\sigma}, \phi_{j i}, \ldots$ this reads

$$
\begin{aligned}
\ldots= & \operatorname{Ad}\left(\left(\left\langle\hat{\sigma}\left(. .+\hat{g}_{j i}\right)-\hat{\sigma}(. .), \sigma\left(g_{j i}+z\right)\right\rangle \otimes \mathbb{1} \otimes \mathbb{1}\right)\right. \\
& \left(\lambda_{\widehat{G} / N^{\perp}}\left(-\hat{g}_{j i}\right) \otimes \mathbb{1} \otimes \mathbb{1}\right)\left(\left\langle\hat{\sigma}\left(\hat{g}_{j i}-. .\right), \sigma\left(-+g_{j i}\right)-\sigma(-)\right\rangle \otimes \mathbb{1}\right) \\
& \left.\left(\mathbb{1} \otimes \lambda_{G / N}\left(-g_{j i}\right) \otimes \mathbb{1}\right)\left(\mathbb{1} \otimes \bar{\mu}_{j}\left(-\sigma(-+z), g_{j i}+z\right)\right)\right) \\
& \left(\mathbb{1} \otimes \mathbb{1} \otimes \zeta_{j i}(z)\right) \operatorname{Ad}\left(\left(\mathbb{1} \otimes \bar{\mu}_{i}(-\sigma(.+z), z)\right)\left(\mathbb{1} \otimes\left\langle\hat{\sigma}\left(\hat{g}_{j i}\right), \sigma(.+z)-\sigma(-)\right\rangle \otimes \mathbb{1}\right)\right. \\
& \left(\left\langle\hat{\sigma}\left(\hat{g}_{j i}-. .\right)+\hat{\sigma}(. .)-\hat{\sigma}\left(\hat{g}_{j i},-\sigma\left(g_{j i}+z\right)+\sigma(z)\right\rangle \otimes \mathbb{1} \otimes \mathbb{1}\right)\right),
\end{aligned}
$$

and after some intermediate steps we find

$\hat{\hat{\zeta}}_{j i}(u)(z)=\eta_{j}(u)\left(g_{j i}(u)+z\right)^{-1} \operatorname{Ad}\left(\lambda_{\widehat{G} / N^{\perp}}\left(-\hat{g}_{j i}(u)\right)\right) \otimes \mathbb{1} \otimes \zeta_{j i}(u)(z) \eta_{i}(u)(z)$,

wherein

$$
\begin{gathered}
\eta_{i}(u)(z):=\operatorname{Ad}((\langle\hat{\sigma}(. .)+\hat{\sigma}(-. .), \sigma(z)\rangle\langle\hat{\sigma}(-. .), \sigma(--z)-\sigma(-)\rangle \otimes \mathbb{1} \otimes \mathbb{1}) \\
\left.\left(\mathbb{1} \otimes \bar{\mu}_{i}(u)\left(-\sigma\left({ }_{-}\right), z\right)^{-1}\right)\left(\mathbb{1} \otimes \lambda_{G / N}(z) \otimes \mathbb{1}\right)\right) .
\end{gathered}
$$

This already proves part of the second half of the theorem, for we see that the double dual is isomorphic to a pair with transition functions

$$
(u, z) \mapsto \operatorname{Ad}\left(\lambda_{\widehat{G} / N^{\perp}}\left(-\hat{g}_{j i}(u)\right)\right) \otimes \mathbb{1} \otimes \zeta_{j i}(u)(z) .
$$

For general reasons such a pair is isomorphic to a pair with transition function $\zeta_{j i}$, for

$$
(u, z) \mapsto \lambda_{\widehat{G} / N^{\perp}}\left(-\hat{g}_{j i}(u)\right) \otimes \mathbb{1} \otimes \mathbb{1} \in \mathrm{U}\left(L^{2}\left(\widehat{G} / N^{\perp}\right) \otimes L^{2}(G / N) \otimes \mathcal{H}\right)
$$


is a unitary cocycle. However, we can give a concrete isomorphism. Let us denote by $\mathcal{F}: L^{2}\left(\widehat{G} / N^{\perp}\right) \rightarrow L^{2}(N)$ the Fourier transform, then we obtain a multiplication operator

$$
\mathcal{F} \circ \lambda_{\widehat{G} / N^{\perp}}\left(-\hat{g}_{j i}(u)\right) \circ \mathcal{F}^{-1}=\left\langle\hat{g}_{j i}, .\right\rangle^{-1}=\left.\phi_{j i}(u)(., 0)\right|_{N} \in \mathrm{U}\left(L^{2}(N)\right) .
$$

Thus, by definition of $\phi_{j i}$ we have

$$
\begin{aligned}
& \operatorname{Ad}\left(\lambda_{\widehat{G} / N^{\perp}}\left(-\hat{g}_{j i}(u)\right)\right) \otimes \mathbb{1} \otimes \zeta_{j i}(u)(z) \\
= & \operatorname{Ad}\left(\left.\left(\mathcal{F}^{-1} \otimes \mathbb{1} \otimes \mathbb{1}\right) \circ \bar{\mu}_{j}(u)\left(., g_{j i}(u)+z\right)^{-1}\right|_{N} \circ(\mathcal{F} \otimes \mathbb{1} \otimes \mathbb{1})\right) \\
& \left(\mathbb{1} \otimes \mathbb{1} \otimes \zeta_{j i}(u)(z)\right) \operatorname{Ad}\left(\left.\left(\mathcal{F}^{-1} \otimes \mathbb{1} \otimes \mathbb{1}\right) \circ \bar{\mu}_{i}(u)(., z)\right|_{N} \circ(\mathcal{F} \otimes \mathbb{1} \otimes \mathbb{1})\right),
\end{aligned}
$$

and we let

$$
\vartheta_{i}(u)(z):=\operatorname{Ad}\left(\left.\left(\mathcal{F}^{-1} \otimes \mathbb{1} \otimes \mathbb{1}\right) \circ \bar{\mu}_{i}(u)(., z)\right|_{N} \circ(\mathcal{F} \otimes \mathbb{1} \otimes \mathbb{1})\right) \eta_{i}(u)(z) .
$$

Due to Lemma $3.1(i) \vartheta_{i}$ is continuous. We obtain

$$
\hat{\hat{\zeta}}_{j i}(u)(z)=\vartheta_{j}(u)\left(g_{j i}(u)+z\right)^{-1}\left(\mathbb{1} \otimes \mathbb{1} \otimes \zeta_{j i}(u)(z)\right) \vartheta_{i}(u)(z),
$$

so $\vartheta_{i}$ define local isomorphisms between $\widehat{\widehat{P}}$ and $\mathrm{PU}\left(L^{2}\left(\widehat{G} / N^{\perp}\right) \otimes L^{2}(G / N)\right) \otimes$ $P$ which fit together to a global isomorphism

$$
(\mathbb{1}, \vartheta, \theta):(\widehat{\widehat{P}}, \widehat{\hat{E}}) \rightarrow\left(\operatorname{PU}\left(L^{2}\left(\widehat{G} / N^{\perp}\right) \otimes L^{2}(G / N)\right) \otimes P, E\right) .
$$

We have to compute the behaviour of the double dual decker under this isomorphism, i.e. we have to compute

$$
\left.\mu_{i}^{\prime}(u)(g, z):=\vartheta_{i}(u)(z+g N) \hat{\mu}_{i}(u)(g, z)\right) \vartheta_{i}(u)(z)^{-1},
$$

for $\hat{\hat{\mu}}_{i}(u)(g, z)=\operatorname{Ad}(\langle\hat{\sigma}(.),-g.\rangle \otimes \mathbb{1} \otimes \mathbb{1})$. This yields ${ }^{\circledR}$

$$
\begin{aligned}
\mu_{i}^{\prime}(g, z)= & \operatorname{Ad}\left(\left.\left(\mathcal{F}^{-1} \otimes \mathbb{1} \otimes \mathbb{1}\right) \circ \bar{\mu}_{i}(., z+g N)\right|_{N} \circ(\mathcal{F} \otimes \mathbb{1} \otimes \mathbb{1})\right. \\
& \left(\mathbb{1} \otimes \lambda_{G / N}(g N) \otimes \mathbb{1}\right)(\langle\hat{\sigma}(. .),-g-\sigma(-)+\sigma(-+g N)\rangle \otimes \mathbb{1}) \\
& \left(\mathbb{1} \otimes \bar{\mu}_{i}(\sigma(-+g N)-\sigma(-), z)\right) \\
& \left.\left.\left(\mathcal{F}^{-1} \otimes \mathbb{1} \otimes \mathbb{1}\right) \circ \bar{\mu}_{i}(., z)^{-1}\right|_{N} \circ(\mathcal{F} \otimes \mathbb{1} \otimes \mathbb{1})\right) \\
= & \operatorname{Ad}\left(\left.\left(\mathcal{F}^{-1} \otimes \mathbb{1} \otimes \mathbb{1}\right) \circ \bar{\mu}_{i}(., z+g N)\right|_{N}\right. \\
& \left(\mathbb{1} \otimes \lambda_{G / N}(g N) \otimes \mathbb{1}\right)\left(\lambda_{N}(g+\sigma(-)-\sigma(-+g N)) \otimes \mathbb{1}\right) \\
& \left(\mathbb{1} \otimes \bar{\mu}_{i}(\sigma(-+g N)-\sigma(-), z)\right) \\
& \left.\left.\bar{\mu}_{i}(., z)^{-1}\right|_{N} \circ(\mathcal{F} \otimes \mathbb{1} \otimes \mathbb{1})\right) \\
= & \operatorname{Ad}\left(\left(\mathcal{F}^{-1} \otimes \mathbb{1} \otimes \mathbb{1}\right) \circ\left(\mathbb{1} \otimes \lambda_{G / N}(g N) \otimes \mathbb{1}\right)\right. \\
& \left(\lambda_{N}(g+\sigma(-)-\sigma(-+g N)) \otimes \mathbb{1}\right) \circ(\mathcal{F} \otimes \mathbb{1} \otimes \mathbb{1}) \\
& \left.\left(\mathbb{1} \otimes \mathbb{1} \otimes \bar{\mu}_{i}(g, z)\right)\right) .
\end{aligned}
$$


The last equality is just the cocycle condition for $\bar{\mu}_{i}$. We can make a further manipulation by use of the following isomorphism

$$
\mathcal{S}: L^{2}(N) \otimes L^{2}(G / N) \cong L^{2}(N \times G / N) \rightarrow L^{2}(G) .
$$

We let $(\mathcal{S}(f))(g):=f(g-\sigma(g N), g N)$. Its inverse is given by $\left(\mathcal{S}^{-1}(f)\right)(n, z):=$ $f(n+\sigma(z))$, and it is immediate to verify that

$$
\mathcal{S}^{-1} \circ \lambda_{G}(g) \circ \mathcal{S}=\lambda_{G / N}(g N) \lambda_{N}\left(g+\sigma\left(_{-}\right)-\sigma\left({ }_{-}+g N\right)\right) .
$$

This implies for the cocycle that

$$
\begin{aligned}
\mu_{i}^{\prime}(u)(g, z)= & \operatorname{Ad}\left(\left(\mathcal{F}^{-1} \otimes \mathbb{1} \otimes \mathbb{1}\right) \circ(\mathcal{S} \otimes \mathbb{1}) \circ\left(\lambda_{G}(g) \otimes \bar{\mu}_{i}(u)(g, z)\right)\right. \\
& \circ(\mathcal{S} \otimes \mathbb{1}) \circ(\mathcal{F} \otimes \mathbb{1} \otimes \mathbb{1}))
\end{aligned}
$$

To summarise, we have shown that there is an isomorphism of dynamical triples

$$
((\mathcal{S} \otimes \mathbb{1}) \circ(\mathcal{F} \otimes \mathbb{1} \otimes \mathbb{1}), \vartheta, \theta):(\hat{\hat{\rho}}, \widehat{\hat{P}}, \widehat{\widehat{E}}) \rightarrow\left(\left(\operatorname{Ad} \circ \lambda_{G}\right) \otimes \rho, \operatorname{PU}\left(L^{2}(G)\right) \otimes P, E\right) .
$$

So, as we discussed in Example 2.2, the double dual triple is outer conjugate to the triple we started with.

We finally comment on the naturality of the defined maps. Let $f: B^{\prime} \rightarrow B$ be a map of bases spaces. Then we have a diagram

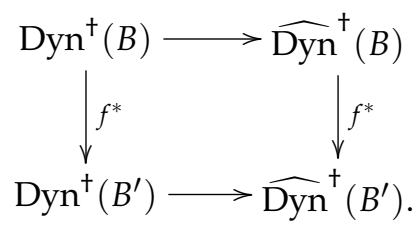

On the local level pullback with $f^{*}$ is the purely formal substitution of $u \in B$ by $f\left(u^{\prime}\right)$ for $u^{\prime} \in B^{\prime}$ in all formulas, and it follows that the diagram commutes.

This proves the theorem.

\subsection{The Relation to Topological T-Duality}

So far we have found that dualisable dynamical triples and dualisable dual dynamical triples have the same isomorphism classes. We demonstrate that the theory developed so far is intimately connected to topological T-duality.

Let $(\rho, P, E)$ be dualisable and $\widehat{P}, \widehat{E}$ as above. Let $P_{\text {top }}:=\operatorname{PU}\left(L^{2}(G / N)\right) \otimes P$.

Theorem 3.3 The two pairs $\left(P_{\text {top }}, E\right)$ and $(\widehat{P}, \widehat{E})$ with underlying Hilbert space $\widehat{\mathcal{H}}=L^{2}(G / N) \otimes \mathcal{H}$ span a topological triple $\left(\kappa^{\text {top }},\left(P_{\text {top }}, E\right),(\widehat{P}, \widehat{E})\right)$, and we obtain a map

$$
\tau(B): \operatorname{Dyn}^{\dagger}(B) \rightarrow \operatorname{Top}(B) .
$$


Moreover, this map is natural, i.e. it defines a natural transformation of functors

$$
\tau: \mathrm{Dyn}^{\dagger} \rightarrow \text { Top. }
$$

Proof : We have to show that there exists (a natural choice of) an isomorphism $\kappa^{\text {top }}: E \times_{B} \widehat{P} \rightarrow P_{\text {top }} \times_{B} \widehat{E}$ which satisfies the Poincaré condition. We are going to define $\kappa^{\text {top }}$ by local isomorphisms of the locally trivialised pairs. Let

$$
\kappa_{i}^{\text {top }}: U_{i} \rightarrow \operatorname{Map}\left(G / N \times \widehat{G} / N^{\perp}, \operatorname{PU}\left(L^{2}(G / N) \otimes \mathcal{H}\right)\right)
$$

be given by the formula

$$
\kappa_{i}^{\mathrm{top}}(u)(z, \hat{z}):=\operatorname{Ad}(\underbrace{\left(\bar{\kappa}^{\sigma}(z, \hat{z}) \otimes \mathbb{1}\right) \bar{\mu}_{i}(u)(-\sigma(-), z)^{-1}\left(\lambda_{G / N}(z) \otimes \mathbb{1}\right)}_{=: \bar{\kappa}_{i}^{\mathrm{top}}(u)(z, \hat{z})})
$$

$\kappa_{i}^{\text {top }}$ is continuous, for the first factor is exactly $\kappa^{\sigma}$ of Lemma 2.3 which defines the Poincaré class, thus it is continuous. The left regular representation $\lambda_{G / N}$ is continuous and by Lemma $3.1($ iii $)(u, z) \mapsto \operatorname{Ad}\left(\bar{\mu}_{i}(u)(-\sigma(-), z)\right)$ is continuous. The functions $(u, z, \hat{z}) \mapsto \mathbb{1} \otimes \zeta_{j i}(u)(z)$ and $(u, z, \hat{z}) \mapsto \mathbb{1} \otimes \hat{\zeta}_{j i}(u)(\hat{z})$ are transition functions for the bundles $P_{\text {dyn }} \times_{B} \widehat{E}$ and $E \times_{B} \widehat{P}$, and the functions $\kappa_{i}^{\text {top }}$ will define a global isomorphism if and only if

$$
\kappa_{j}^{\mathrm{top}}\left(u, g_{j i}(u)+z, \hat{g}_{j i}(u)+\hat{z}\right) \hat{\zeta}_{j i}(u)(\hat{z}) \kappa_{i}^{\mathrm{top}}(u, z, \hat{z})^{-1}=\mathbb{1} \otimes \zeta_{j i}(u)(z),
$$

for $(u, z, \hat{z}) \in U_{i j} \times G / N \times \widehat{G} / N^{\perp}$. By construction, the Poincaré condition will be satisfied automatically.

We are able to do this calculation on the projective unitary level directly, but later we need the same calculation on the unitary level, so we prepare ourselves first with a choice of lifts $\overline{\hat{\zeta}}_{j i}$.

Recall that $u \mapsto \operatorname{Ad}\left(\phi_{j i}(u)(-\sigma(-), 0)\right)$ is continuous. If we pass once more to a refined atlas which again we denote by $\left\{U_{i}\right\}$, then there exist continuous lifts

$$
\bar{\phi}_{j i}: U_{j i} \rightarrow L^{\infty}(G / N, \mathrm{U}(1))
$$

such that $\left.\operatorname{Ad}\left(\bar{\phi}_{j i}(u)\right)=\operatorname{Ad}\left(\phi_{j i}(u)\left(-\sigma()_{-}\right), 0\right)\right)$; by those we define lifted transition functions functions $\overline{\hat{\zeta}}_{j i}: U_{j i} \rightarrow L^{\infty}\left(\widehat{G} / N^{\perp}, L^{\infty}(G / N, \mathrm{U}(\mathcal{H}))\right)$ by

$$
\begin{gathered}
\bar{\zeta}_{j i}(u)(\hat{z}):=\left(\bar{\kappa}^{\sigma}\left(-g_{j i}(u), \hat{g}_{j i}(u)+\hat{z}\right) \otimes \mathbb{1}_{\mathcal{H}}\right)\left(\lambda_{G / N}\left(-g_{j i}(u)\right) \otimes \mathbb{1}_{\mathcal{H}}\right) \\
\bar{\zeta}_{j i}(u)\left(-_{-}\right)\left(\bar{\phi}_{j i}(u)^{-1} \otimes \mathbb{1}_{\mathcal{H}}\right),
\end{gathered}
$$

for $u \in U_{i j}, \hat{z} \in \widehat{G} / N^{\perp}$. 


$$
\begin{aligned}
\text { Now, we calculate }{ }^{\mathbb{1}} & \\
& \bar{\kappa}_{j}^{\text {top }}\left(g_{j i}+z, \hat{g}_{j i}+\hat{z}\right) \bar{\zeta}_{j i}(\hat{z}) \bar{\kappa}_{i}^{\mathrm{top}}(z, \hat{z})^{-1} \\
= & \left(\left\langle\hat{\sigma}\left(\hat{g}_{j i}+\hat{z}\right),-\sigma\left({ }_{-}\right)+\sigma\left(--g_{j i}-z\right)\right\rangle \otimes \mathbb{1}\right) \bar{\mu}_{j}\left(-\sigma\left(_{-}\right), g_{j i}+z\right)^{-1} \\
& \left(\lambda_{G / N}\left(g_{j i}+z\right) \otimes \mathbb{1}\right) \\
& \left(\left\langle\hat{\sigma}\left(\hat{g}_{j i}+\hat{z}\right),-\sigma\left({ }_{-}\right)+\sigma\left(-+g_{j i}\right)\right\rangle \otimes \mathbb{1}\right)\left(\lambda_{G / N}\left(-g_{j i}\right) \otimes \mathbb{1}\right) \\
& \bar{\zeta}_{j i}\left(-_{-}\right)\left(\bar{\phi}_{j i}^{-1} \otimes \mathbb{1}\right) \\
& \left(\lambda_{G / N}(-z) \otimes \mathbb{1}\right) \bar{\mu}_{i}(-\sigma(-), z)(\langle\hat{\sigma}(\hat{z}), \sigma(-)-\sigma(--z)\rangle \otimes \mathbb{1}) \\
= & \left(\left\langle\hat{\sigma}\left(\hat{g}_{j i}+\hat{z}\right)-\hat{\sigma}(\hat{z}),-\sigma(-)+\sigma(-z)\right\rangle \otimes \mathbb{1}\right) \\
& \bar{\mu}_{j}\left(-\sigma(-), g_{j i}+z\right)^{-1} \bar{\zeta}_{j i}\left(z-{ }_{-}\right)\left(\bar{\phi}_{j i}(--z)^{-1} \otimes \mathbb{1}\right) \bar{\mu}_{i}(-\sigma(-), z) \\
= & \left(\left\langle\hat{\sigma}\left(\hat{g}_{j i}+\hat{z}\right)-\hat{\sigma}(\hat{z}),-\sigma(-)+\sigma(-z)+\sigma(z)\right\rangle \otimes \mathbb{1}\right)\left\langle\hat{\sigma}\left(\hat{g}_{j i}+\hat{z}\right)-\hat{\sigma}(\hat{z}),-\sigma(z)\right\rangle \\
& \bar{\mu}_{j}(-\sigma(-))\left(u, g_{j i}+z\right)^{-1} \bar{\zeta}_{j i}\left(z-{ }_{-}\right)\left(\bar{\phi}_{j i}(--z)^{-1} \otimes \mathbb{1}\right) \bar{\mu}_{i}(-\sigma(-), z) \\
= & \left(\left\langle\hat{g}_{j i},-\sigma(-)+\sigma(--z)+\sigma(z)\right\rangle \otimes \mathbb{1}\right)\left\langle\hat{\sigma}\left(\hat{g}_{j i}+\hat{z}\right)-\hat{\sigma}(\hat{z}),-\sigma(z)\right\rangle \\
& \bar{\mu}_{j}\left(-\sigma(-), g_{j i}+z\right)^{-1} \bar{\zeta}_{j i}\left(z-{ }_{-}\right)\left(\bar{\phi}_{j i}(--z)^{-1} \otimes \mathbb{1}\right) \bar{\mu}_{i}(-\sigma(-), z) .
\end{aligned}
$$

The variable $\hat{z}$ only occurs in $\left\langle\hat{\sigma}\left(\hat{g}_{j i}+\hat{z}\right)-\hat{\sigma}(\hat{z}),-\sigma(z)\right\rangle$. To the remaining expressinon we can apply equation (17) of Lemma 3.1 which is valid on the unitary level up to a $\mathrm{U}(1)$-valued perturbation $\alpha_{j i}^{\prime}: U_{j i} \rightarrow L^{\infty}(G / N, \mathrm{U}(1))$. We obtain

$$
\ldots=\underbrace{\left\langle\hat{\sigma}\left(\hat{g}_{j i}(u)+\hat{z}\right)-\hat{\sigma}(\hat{z}),-\sigma(z)\right\rangle \alpha_{j i}^{\prime}(u)(z)^{-1}}_{=: \alpha_{j i}(u)(z, \hat{z})^{-1} \in \mathrm{U}(1)}\left(\mathbb{1} \otimes \bar{\zeta}_{j i}(u)(z)\right) .
$$

This establishes the existence of $\kappa^{\text {top }}$.

It remains to check that the constructed equivalence class of the topological triple only depends on the equivalence class of the dynamical triple, but this not difficult to establish. We just mention that if we start with an exterior equivalent decker which differs locally by $c_{i}: U_{i} \times G \times G / N \rightarrow \mathrm{U}(\mathcal{H})$ from the cocycle $\mu_{i}$, then $\kappa^{\text {top }}$ differs by the null-homotopic bundle automorphism which is locally of the form $c_{i}(u,-\sigma(-+z), z)$.

Finally, we remark that $\tau$ is natural. Indeed, let $f: B^{\prime} \rightarrow B$ be a map of bases spaces. Then there is a commutative diagram

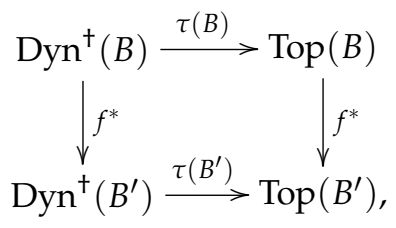

because in our local construction pullback with $f$ is just pullback of the underlying locally defined functions by $f$ which corresponds to the formal substitution of $u \in B$ by $f\left(u^{\prime}\right)$ for $u^{\prime} \in B^{\prime}$ in all formulas. 
Our aim is to construct an inverse of $\tau(B): \operatorname{Dyn}^{\dagger}(B) \rightarrow \operatorname{Top}(B)$. In particular, we wish to construct from the data of a topological triple

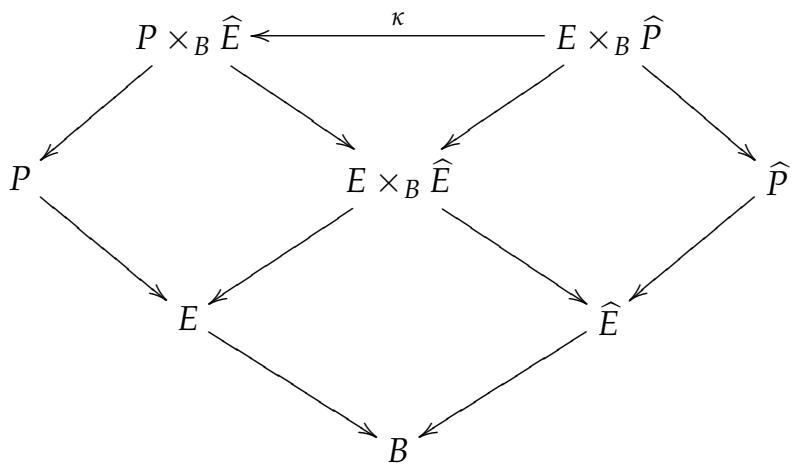

a decker $\rho$ on (a pair stably isomorphic to) $(P, E)$. The space $E \times_{B} \widehat{P}$ clearly has a $G / N$-action, and by use of $\kappa$ we can define a $G / N$-action on $P \times{ }_{B} \widehat{E}$. The leading idea is that the Poincaré condition enables us to push forward this action down to a $G$-action on $P$. We will see that this fails in general as an obstruction against a decker occurs.

Let $(\kappa,(P, E),(\widehat{P}, \widehat{E}))$ be a topological triple over $B$, and let $g_{i j}, \zeta_{i j}$ and $\hat{g}_{i j}, \hat{\zeta}_{i j}$ be transition functions for the pairs $(P, E)$ and $(\widehat{P}, \widehat{E})$. Without restriction we let $\widehat{\mathcal{H}}=L^{2}(G / N) \otimes \mathcal{H}$ be the underlying Hilbert space. The existence of $\kappa$ implies the existence of locally defined $\kappa_{i}: U_{i} \rightarrow \operatorname{Map}\left(G / N \times \widehat{G} / N^{\perp}, \mathrm{PU}(\widehat{\mathcal{H}})\right)$ such that

$$
\kappa_{j}(u)\left(g_{j i}(u)+z, \hat{g}_{j i}(u)+\hat{z}\right) \hat{\zeta}_{j i}(u)(\hat{z}) \kappa_{i}(u)(z, \hat{z})^{-1}=\zeta_{j i}(u)(z) .
$$

Since $\kappa$ satisfies the Poincaré condition each $\kappa_{i}$ can be written in the form

$$
\kappa_{i}(u)(z, \hat{z})=\kappa_{i}^{a}(u)(z) \kappa^{\sigma}(z, \hat{z}) \operatorname{Ad}\left(v_{i}(u, z, \hat{z})\right) \kappa_{i}^{b}(u)(\hat{z}),
$$

wherein $\kappa^{\sigma}$ is from Lemma 2.3 and $\kappa_{i}^{a}, \kappa_{i}^{b}$ are some continuous projective unitary functions and $v_{i}: U_{i} \times G / N \times \widehat{G} / N^{\perp} \rightarrow \mathrm{U}(\widehat{\mathcal{H}})$ is a continuous unitary function. We introduce some short hands to get rid of $\kappa_{i}^{a}$ and $\kappa_{i}^{b}$. We let $\zeta_{j i}^{\prime}(u)(z):=\kappa_{j}^{a}(u)\left(g_{j i}(u)+z\right)^{-1} \zeta_{j i}(u)(z) \kappa_{i}^{a}(u)(z)$ and analogously $\hat{\zeta}_{j i}^{\prime}(u)(\hat{z}):=$ $\kappa_{j}^{b}(u)\left(g_{j i}(u)+z\right) \hat{\zeta}_{j i}(u)(z) \kappa_{i}^{b}(u)(z)^{-1}$. Thus we have ${ }^{\circledR}$

$$
\begin{aligned}
\zeta_{j i}^{\prime}(z)= & \kappa^{\sigma}\left(g_{j i}+z, \hat{g}_{j i}+\hat{z}\right) \operatorname{Ad}\left(v_{j}\left(g_{j i}+z, \hat{g}_{j i}+\hat{z}\right)\right) \\
& \hat{\zeta}_{j i}^{\prime}(\hat{z}) \operatorname{Ad}\left(v_{i}(z, \hat{z})\right)^{-1} \kappa^{\sigma}(z, \hat{z})^{-1} .
\end{aligned}
$$

Let us choose, if necessary after a refinement of the given atlas, lifts $\bar{\zeta}_{j i}^{\prime}: U_{i j} \rightarrow$ $L^{\infty}(G / N, \mathrm{U}(\widehat{\mathcal{H}}))$ and ${\overline{\hat{\zeta}^{\prime}}}_{j i}: U_{i j} \rightarrow L^{\infty}\left(\widehat{G} / N^{\perp}, \mathrm{U}(\widehat{\mathcal{H}})\right)$ of the transition functions 
(Lemma A.8). Then we obtain ${ }^{(1)}$

$$
\begin{aligned}
\bar{\zeta}_{j i}^{\prime}(z)= & \left(\bar{\kappa}^{\sigma}\left(g_{j i}+z, \hat{g}_{j i}+\hat{z}\right) \otimes \mathbb{1}\right) v_{j}\left(u, g_{j i}+z, \hat{g}_{j i}+\hat{z}\right) \\
& \overline{\hat{\zeta}}_{j i}(\hat{z}) v_{i}(z, \hat{z})^{-1}\left(\bar{\kappa}^{\sigma}(z, \hat{z})^{-1} \otimes \mathbb{1}\right) \alpha_{j i}(z, \hat{z}),
\end{aligned}
$$

for some continuous $\alpha_{j i}: U_{i j} \rightarrow L^{\infty}\left(G / N \times \widehat{G} / N^{\perp}, \mathrm{U}(1)\right)$ and $\bar{\kappa}^{\sigma}(z, \hat{z}):=$ $\left\langle\hat{\sigma}(\hat{z}), \sigma\left({ }_{-}-z\right)-\sigma(-)\right\rangle \in L^{\infty}(G / N, \mathrm{U}(1)) \subset \mathrm{U}(\widehat{\mathcal{H}}) ; \sigma$ and $\hat{\sigma}$ are both chosen to be Borel. By direct computation, it follows that

$$
\left(\delta_{g \times \hat{g}} \alpha_{. .}\right)_{k j i}(u)(z, \hat{z})=\psi_{k j i}(u)(z) \hat{\psi}_{k j i}(u)(\hat{z})^{-1},
$$

for the twisted Čech cocycles $\psi \ldots . \mathbb{1}:=\delta_{g} \bar{\zeta}_{. .}^{\prime}$ and $\hat{\psi}_{\ldots} \cdot \mathbb{1}:=\delta_{\hat{g}} \overline{\hat{\zeta}}_{\text {... }}$. It is our task now to use the Poincaré condition of the isomorphism $\kappa$ to deduce a more concrete formula for $\alpha_{j i}$ from which we can extract the existence of a decker. It will be sufficient to investigate the structure of

$$
A_{j i}(u, z):=\operatorname{Ad}\left(\alpha_{j i}(u)(z, . .)\right) \in \mathrm{P} L^{\infty}\left(\widehat{G} / N^{\perp}, \mathrm{U}(1)\right) .
$$

We let

$$
\beta_{j i}(u, z):=\operatorname{Ad}\left(\left\langle\hat{\sigma}\left(. .+\hat{g}_{j i}(u)\right)-\hat{\sigma}(. .), \sigma\left(g_{j i}(u)+z\right)\right\rangle\right) \in \mathrm{P} L^{\infty}\left(\widehat{G} / N^{\perp}, \mathrm{U}(1)\right)
$$

Note for the next lemma that $\beta_{j i}(u, z)$ equals $\hat{\kappa}^{\hat{\sigma}}\left(g_{j i}(u)+z, \hat{g}_{j i}(u)\right)^{-1}$ up to conjugation by the unitary implemented $\operatorname{Ad}\left(\lambda_{\widehat{G} / N^{\perp}}\left(\hat{g}_{j i}(u)\right)\right)$, so the Čech classes $\left[\beta_{j i}\right]$ and $\left[(u, z) \mapsto \hat{\kappa}^{\hat{\sigma}}\left(g_{j i}(u)+z, \hat{g}_{j i}(u)\right)^{-1}\right]$ in $\check{H}^{1}\left(U_{j i} \times G / N, \underline{\mathrm{U}(1)}\right)$ defined by these functions agree. (Cp. Lemma 2.4)

The Hilbert space $\mathcal{H}$ occurs in the following to force that certain operators which are defined on different tensor factors of $\mathcal{H} \otimes L^{2}\left(\widehat{G} / N^{\perp}\right) \otimes \widehat{\mathcal{H}}$ commute.

Lemma 3.2 $A_{j i}$ is continuous, and there are continuous functions ${ }^{12}$

$\left.w_{j i}: U_{j i} \rightarrow \operatorname{Map}\left(G / N, L^{\infty}\left(\widehat{G} / N^{\perp}, \mathrm{U}^{\mathrm{ab}}(\mathcal{H})\right)\right)\right) \subset \operatorname{Map}\left(G / N, \mathrm{U}\left(\mathcal{H} \otimes L^{2}\left(\widehat{G} / N^{\perp}\right)\right)\right)$

and

$$
\gamma_{j i}: U_{j i} \rightarrow \mathrm{PU}^{\mathrm{ab}}(\mathcal{H})
$$

such that

$$
\mathbb{1}_{\mathcal{H}} \otimes A_{j i}(u, z)=\mathbb{1}_{\mathcal{H}} \otimes \beta_{j i}(u, z) \operatorname{Ad}\left(w_{j i}(u)(z)\right)\left(\gamma_{j i}(u) \otimes \mathbb{1}_{L^{2}\left(\widehat{G} / N^{\perp}\right)}\right) .
$$

${ }^{12} \mathrm{U}^{\mathrm{ab}}(\mathcal{H})$ is a commutative, contractible subgroup of $\mathrm{U}(\mathcal{H})$ such that $\mathrm{U}(1) \cdot \mathbb{1} \subset \mathrm{U}^{\mathrm{ab}}(\mathcal{H})$. See section A.3, page 92. 
Proof : Equation (25) implies

$$
\begin{aligned}
\mathbb{1} \otimes \zeta_{j i}^{\prime}(u)(z)= & \operatorname{Ad}\left(\mathbb{1}_{L^{2}\left(\widehat{G} / N^{\perp}\right)} \otimes \bar{\zeta}_{j i}(u)(z)\right) \\
= & \operatorname{Ad}\left(\left(\bar{\kappa}^{\sigma}\left(g_{j i}(u)+z, \hat{g}_{j i}(u)+. .\right) \otimes \mathbb{1}_{\mathcal{H}}\right) v_{j}\left(u, g_{j i}(u)+z, \hat{g}_{j i}(u)+. .\right)\right. \\
& \left.\left.{\overline{\zeta^{\prime}}}_{j i}(u)(. .) v_{i}(u, z, . .)\right)^{-1}\left(\overline{\mathcal{K}}^{\sigma}(z, . .)^{-1} \otimes \mathbb{1}_{\mathcal{H}}\right)\right)\left(A_{j i}(u)(z) \otimes \mathbb{1}_{\widehat{\mathcal{H}}}\right) \\
& \left.\in \operatorname{PU}\left(L^{2}\left(\widehat{G} / N^{\perp}\right) \otimes \widehat{\mathcal{H}}\right)\right) .
\end{aligned}
$$

The terms inside the bracket are continuous unitary functions on $U_{j i} \times G / N$. Thus we have equality of the Čech classes $\left[\zeta_{j i}^{\prime}\right]=\left[\mathbb{1} \otimes \zeta_{j i}^{\prime}\right]=\left[A_{j i} \otimes \mathbb{1}\right]=\left[A_{j i}\right] \in$ $\check{H}^{1}\left(U_{j i} \times G / N, \underline{\mathrm{U}(1)}\right)$. We compute the Čech class of $\mathbb{1} \otimes \zeta_{j i}^{\prime}$ by equation (24) for $\hat{z}=0$. Then $\kappa^{\sigma}(z, 0)=\mathbb{1}$, hence has trivial Čech class. The map $(u, z) \mapsto$ $\hat{\zeta}_{j i}^{\prime}(u, 0)$ has Čech class $\left[(u, z) \mapsto \hat{\zeta}_{j i}^{\prime}(u, 0)\right]$. By Remark A.2, let $\gamma_{j i}: U_{j i} \rightarrow$ $\operatorname{PU}^{\mathrm{ab}}(\mathcal{H})$ be such that $\left[(u, z) \mapsto \hat{\zeta}_{j i}^{\prime}(u, 0)\right]=\left[(u, z) \mapsto \gamma_{j i}(u)\right]=\operatorname{pr}_{U_{j i}}^{*}\left[\gamma_{j i}\right]$. Then

$$
\begin{aligned}
{\left[\mathbb{1} \otimes \zeta_{j i}^{\prime}\right] } & =\left[(u, z) \mapsto \kappa^{\sigma}\left(g_{j i}(u)+z, \hat{g}_{j i}(u)\right)\right]+\left[u \mapsto \hat{\zeta}_{j i}^{\prime}(u, 0)\right] \\
& =\left(\left(g_{j i} \circ \operatorname{pr}_{U_{j i}}+\operatorname{pr}_{G / N}\right) \times\left(\hat{g}_{j i} \circ \operatorname{pr}_{U_{j i}}\right)\right)^{*} \pi+\operatorname{pr}_{U_{j i}}^{*}\left[\gamma_{j i}\right] \\
& =\left[\beta_{j i}\right]+\operatorname{pr}_{U_{j i}}^{*}\left[\gamma_{j i}\right] .
\end{aligned}
$$

This implies that $A_{j i} \otimes \mathbb{1}_{\mathcal{H}}$ equals $\beta_{j i} \otimes \gamma_{j i}$ up to Ad of a continuous unitary function $w_{j i}: U_{j i} \times G / N \rightarrow \mathrm{U}\left(L^{2}\left(\widehat{G} / N^{\perp}, \mathcal{H}\right)\right)$. $w_{j i}$ takes values in the subgroup $L^{\infty}\left(\widehat{G} / N^{\perp}, \mathrm{U}^{\mathrm{ab}}(\widehat{\mathcal{H}})\right)$ only, since $A_{j i}, \beta_{j i}$ and $\gamma_{j i}$ are maps into the subgroup $\mathrm{P} L^{\infty}\left(\widehat{G} / N^{\perp}, \mathrm{U}^{\mathrm{ab}}(\widehat{\mathcal{H}})\right) \subset \mathrm{PU}\left(L^{2}\left(\widehat{G} / N^{\perp}, \widehat{\mathcal{H}}\right)\right)$.

Finally, we just interchange the order of the Hilbert spaces in the tensor product $L^{2}\left(\widehat{G} / N^{\perp}\right) \otimes \mathcal{H}$ to $\mathcal{H} \otimes L^{2}\left(\widehat{G} / N^{\perp}\right)$. This will be convenient later.

Remark 3.2 By passing to a refined atlas, we can always achieve that $\gamma_{j i}=\mathbb{1}$, for we can apply Lemma A.8 to the functions $U_{j i} \ni u \mapsto \hat{\zeta}_{j i}^{\prime}(u)(0)$. In that case we have $w_{j i}(u)(z)(\hat{z}) \in \mathrm{U}(1)$.

It is important to note that although $w_{j i}$ depends on the choice of $\gamma_{j i}$ the term $\left(d_{*} w_{. .}\right)_{j i}(u)=d\left(w_{j i}(u)\right)$ does not; here $d$ is the (first) boundary operator of group cohomology

$$
\begin{aligned}
\operatorname{Map}\left(G / N, L^{\infty}\left(\widehat{G} / N^{\perp}, \mathrm{U}^{\mathrm{ab}}(\mathcal{H})\right)\right) & \stackrel{d}{\longrightarrow} \operatorname{Map}\left(G, \operatorname{Map}\left(G / N, L^{\infty}\left(\widehat{G} / N^{\perp}, \mathrm{U}^{\mathrm{ab}}(\mathcal{H})\right)\right)\right) \\
w & \mapsto\left((h, z) \mapsto w(z+h N)(. .) w(z)(. .)^{-1}\right)
\end{aligned}
$$

The sheaf of continuous functions into the range (or source) of $d$ is a $\underline{G} / N \times \widehat{G} / N^{\perp}$ module in the obvious way. So the following statement is meaningful for the twisted Čech boundary operator $\delta_{g . .} \times \hat{g} .$.

Lemma 3.3 $\operatorname{Ad}\left(\left(\delta_{g . . \times \hat{g}_{. .}} d_{*} w_{. .}\right)_{k j i}(u)(h, z)(.).\right)=\mathbb{1}$, for all $u \in U_{k j i}, z \in G / N, h \in$ G. 
Proof : Since $d_{*}$ and $\delta_{g . .} \times \hat{g} .$. commute, we have to show that the expression $\operatorname{Ad}\left(\left(\delta_{g . .} \times \hat{g}_{. .} w_{. .}\right)_{k j i}(u)(z)(.).\right)$ is independent of $z$. Because $\left.\operatorname{Ad}\left(\left(\delta_{g \times \hat{g}} \alpha . .\right)\right)_{k j i}(u)(z, .).\right)=$ $\operatorname{Ad}\left(\hat{\psi}_{k j i}(u)(. .)^{-1}\right)$ and $\gamma_{j i}(u)$ are independent of $z$ we have to compute $\delta_{g . .} \times \hat{g}_{. .} \beta_{. .}$. This yields $\left.{ }^{(}\right)$

$$
\begin{aligned}
& \left(\delta_{g_{. .}} \times \hat{g}_{. .} \beta_{. .}\right)_{k j i}(z) \\
= & \operatorname{Ad}\left(\left\langle\hat{\sigma}\left(. .+\hat{g}_{j i}\right)-\hat{\sigma}(. .),-\sigma\left(g_{j i}+z\right)\right\rangle\right. \\
& \left\langle\hat{\sigma}\left(. .+\hat{g}_{k i}\right)-\hat{\sigma}(. .), \sigma\left(g_{k i}+z\right)\right\rangle \\
& \left.\left\langle\hat{\sigma}\left(. .+\hat{g}_{j i}+\hat{g}_{k j}\right)-\hat{\sigma}\left(. .+\hat{g}_{j i}\right),-\sigma\left(g_{k i}+z\right)\right\rangle\right) \\
= & \operatorname{Ad}\left(\left\langle\hat{\sigma}\left(. .+\hat{g}_{j i}\right)-\hat{\sigma}(. .), \sigma\left(g_{k i}\right)-\sigma\left(g_{j i}\right)\right\rangle\right),
\end{aligned}
$$

and the lemma is proven.

It follows that

$$
\varphi_{k j i}(u)(h, z):=\left(\delta_{g . .} \times \hat{g}_{. .} d_{*} w_{. .}\right)_{k j i}(u)(h, z)(. .) \in \mathrm{U}(1)
$$

satisfies $\delta_{g} \varphi=1$ and $d_{*} \varphi_{j i}=1$, i.e. it defines a twisted Čech 2-class

$$
\left[\varphi_{\ldots . .}\right] \in \check{H}^{2}\left(B, \underline{Z_{\text {cont }}^{1}(G, \operatorname{Map}(G / N, U(1)))}, g_{. .}\right) .
$$

Lemma 3.4 The construction of the class $[\varphi$...] defines a natural map

$$
\operatorname{Top}(E, B) \rightarrow \check{H}^{2}\left(B, Z_{\text {cont }}^{1}(G, \operatorname{Map}(G / N, \mathrm{U}(1))), g_{. .}\right) .
$$

Proof : We must show that the constructed class only depends on the isomorphism class of the triple. Let us check all choices in reversed order of their appearance.

Firstly, the choice of $w_{j i}$ is only unique up to a continuous scalar function $U_{j i} \times G / N \rightarrow \mathrm{U}(1)$, so $\varphi_{\ldots} .=\delta_{g \times \hat{g}} d_{*} w_{. .}$changes by a boundary.

Secondly, the choice of $\gamma_{j i}$, is only unique up to a unitary implemented function $U_{j i} \rightarrow \mathrm{U}^{\mathrm{ab}}(\mathcal{H})$, so $w_{j i}$ may change by this function. But this function is independent of $z \in G / N$, so $d_{*} w_{j i}$ does not change.

Thirdly, if we choose different lifts of the transition functions $\zeta_{j i}$, this would change $\alpha_{j i}$ by a function $U_{j i} \rightarrow L^{\infty}(G / N, \mathrm{U}(1))$, so $A_{j i}$ does not change.

Fourthly, if we choose different lifts of the transition functions $\hat{\zeta}_{j i}$, then $\alpha_{j i}$ is changed by a function $U_{j i} \rightarrow L^{\infty}\left(\widehat{G} / N^{\perp}, \mathrm{U}(1)\right)$, so $A_{j i}$, thus $w_{j i}$, changes by a function independent of $z \in G / N$, so $d_{*} w_{j i}$ does not change.

Fifthly, if we choose another atlas for our construction, we can take a common refinement and the normalisation procedure $\zeta_{j i} \mapsto \zeta_{j i}^{\prime}, \hat{\zeta}_{j i} \mapsto \hat{\zeta}_{j i}^{\prime}$, leads to the same equations as above, except we restricted us to the refined atlas, so the class of $\varphi$... is not changed.

Sixthly, if we start with a topological triple which is isomorphic to $(\kappa,(P, E),(\widehat{P}, \widehat{E})$, then, locally, the isomorphisms of the underlying pairs have the same effect as a change of the atlas which does not change the class of $\varphi_{\ldots . . .}$. However, 
we must take care of the homotopy commutativity of diagram (14). So if $\kappa^{\prime}$ is homotopic to $\kappa$, then they differ locally by a continuous unitary function $v_{i}^{\prime}: U_{i} \times G / N \times \widehat{G} / N^{\perp} \rightarrow \mathrm{U}(\widehat{\mathcal{H}})$, and equation (25) becomes

$$
\begin{aligned}
& v_{j}^{\prime}\left(u, g_{j i}(u)+z, \hat{g}_{j i}(u)+\hat{z}\right)^{-1} \bar{\zeta}_{j i}^{\prime}(u)(z) v_{i}^{\prime}(u, z, \hat{z}) \\
= & \left(\bar{\kappa}^{\sigma}\left(g_{j i}(u)+z, \hat{g}_{j i}(u)+\hat{z}\right) \otimes \mathbb{1}\right) v_{j}\left(u, g_{j i}(u)+z, \hat{g}_{j i}(u)+\hat{z}\right) \\
& \overline{\hat{\zeta}}_{j i}(u)(\hat{z}) v_{i}(u, z, \hat{z})^{-1}\left(\bar{\kappa}^{\sigma}(z, \hat{z})^{-1} \otimes \mathbb{1}\right) \alpha_{j i}^{\prime}(u)(z, \hat{z}) .
\end{aligned}
$$

We must investigate how $\alpha_{j i}^{\prime}$ is related to $\alpha_{j i}$. The family $v_{i}^{\prime}$ defines the bundle automorphism $\kappa^{\prime} \circ \kappa^{-1}$ on $P \times_{B} \widehat{E}$ so

$$
\zeta_{j i}^{\prime}(u)(z)\left(v_{i}^{\prime}\left(u, g_{j i}(u)+z, \hat{g}_{j i}(u)+\hat{z}\right)\right)=v_{j}^{\prime}(u, z, \hat{z}) \alpha_{j i}^{\prime \prime}(u)(z, \hat{z}),
$$

for a scalar $\alpha_{j i}^{\prime \prime}: U_{i} \times G / N \times \widehat{G} / N^{\perp} \rightarrow \mathrm{U}(1)$. It follows from the three equations for $\alpha_{j i}, \alpha_{j i}^{\prime}$ and $\alpha_{j i}^{\prime \prime}$ that $\alpha_{j i}^{\prime}=\alpha_{j i} \alpha_{j i}^{\prime \prime}$. So $w_{j i}$ changes by $\alpha_{j i}^{\prime \prime}$. But $\alpha_{. .}^{\prime \prime}$ is a cocycle $\delta_{g \times \hat{g}} \alpha_{. .}^{\prime \prime}=1$ which is easily computed by its definition (29), so $\delta_{g \times \hat{g}} w_{\text {.. }}$ and $\varphi \ldots$ do not change.

Finally, we just remark that the defined map is natural with respect to pullback. I.e. if $f: B^{\prime} \rightarrow B$ is a map of base spaces, then there is a commutative diagram

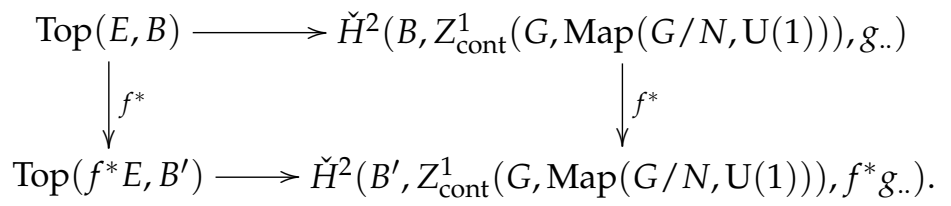

This proves the lemma.

The quotient map $G \rightarrow G / N$ induces a map on the twisted Čech groups

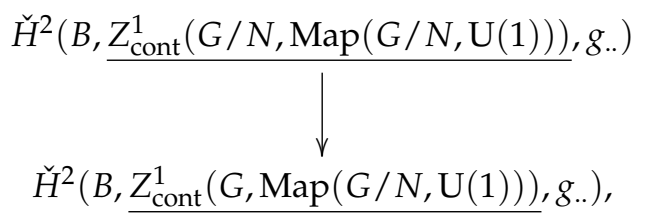

and the map of Lemma 3.4 has an obvious factorisation

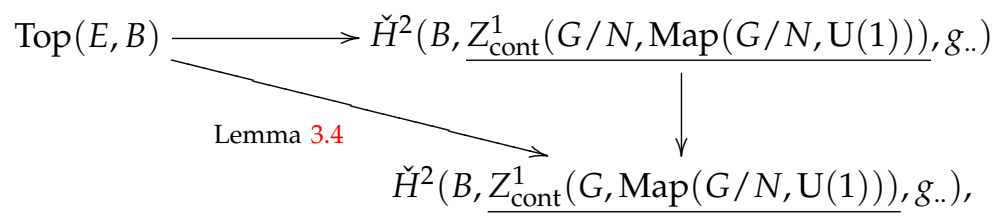


since the definition (27) of $\varphi$... implies

$$
\varphi_{k j i}(u)(h+n, z)=\varphi_{k j i}(u)(h, z),
$$

for all $n \in N$.

Definition 3.2 A topological T-duality triple over B is called almost strict if its Čech class $[\varphi . .$.$] defined by Lemma 3.4$ vanishes. The triple is called strict if its Čech class defined by the horizontal map in the diagram above vanishes already.

We denote by $\operatorname{Top}^{\text {as }}(B)$ respectively $\operatorname{Top}^{\mathrm{s}}(B)$ the set of all almost strict respectively strict topological triples over $B$; so we have obvious inclusions

$$
\operatorname{Top}^{\mathrm{s}}(B) \subset \operatorname{Top}^{\text {as }}(B) \subset \operatorname{Top}(B) .
$$

Remark 3.3 For the class of a topological triple $[(\kappa,(P, E),(\widehat{P}, \widehat{E}))] \in \operatorname{Top}(B)$ its class in $\operatorname{Top}(E, B)$ is only well-defined up to the action of $\operatorname{Aut}_{B}(E)$ on $\operatorname{Top}(E, B)$. However, the the vanishing of the obstruction class in Definition 3.2 is independent of the possible choices, so $\operatorname{Top}^{\text {as }}(B)$ and $\operatorname{Top}^{\mathrm{s}}(B)$ are well-defined.

We will see that strict and almost strict play a major rôle in our theory. The following two lemmata give a first feeling.

Lemma 3.5 The image of the map $\tau(B)$ from dualisable dynamical to topological triples is contained in the set of strict topological triples,

$$
\operatorname{im}(\tau(B)) \subset \operatorname{Top}^{\mathrm{s}}(B)
$$

Proof : In equation (23) we already computed $\alpha_{j i}$ for a topological triple which is constructed out of a dynamical one. (In (23) we did not normalise $\zeta_{j i} \mapsto$ $\zeta_{j i}^{\prime}$, but this does not change $\alpha_{j i}$. $)$ The result is $\alpha_{j i}(u)(z, \hat{z})=\left\langle\hat{\sigma}\left(\hat{g}_{j i}(u)+\hat{z}\right)-\right.$ $\hat{\sigma}(\hat{z}), \sigma(z)\rangle \alpha_{j i}^{\prime}(u)(z)$. So in this case we have

$$
\begin{aligned}
A_{j i}(u)(z) & =\operatorname{Ad}\left(\left\langle\hat{\sigma}\left(\hat{g}_{j i}(u)+. .\right)-\hat{\sigma}(. .), \sigma(z)\right\rangle\right) \\
& =\operatorname{Ad}\left(\left\langle\hat{\sigma}\left(\hat{g}_{j i}(u)+. .\right)-\hat{\sigma}(. .), \sigma\left(g_{j i}(u)+z\right)-\sigma\left(g_{j i}(u)\right\rangle\right)\right. \\
& =\beta_{j i}(u, z) \underbrace{\operatorname{Ad}\left(\left\langle\hat{\sigma}\left(\hat{g}_{j i}(u)+. .\right)-\hat{\sigma}(. .),-\sigma\left(g_{j i}(u)\right\rangle\right)\right.}_{\text {independent of } z \in G / N},
\end{aligned}
$$

i.e. we can choose $w_{j i}$ such that $w_{j i}(u)(z)$ does not depend on $z$. Of course, the choice of $w_{j i}$ is only determined up to a scalar $U_{j i} \times G / N \rightarrow \mathrm{U}(1)$, but in any case $\chi_{j i} \cdot \mathbb{1}:=d_{*} w_{j i}$ defines a function $U_{j i} \rightarrow Z_{\text {cont }}^{1}(G, \operatorname{Map}(G / N, \mathrm{U}(1)))$, so by construction $\varphi_{k j i}=\left(\delta_{g} \chi_{. .}\right)_{k j i}$ is a boundary.

The next (technical) lemma will be the crucial point in the construction of a decker from the data of a topological triple. 
Lemma 3.6 Assume $(\kappa,(P, E),(\widehat{P}, \widehat{E}))$ is almost strict. Then we can find a (sufficiently refined) atlas $\left\{U_{i} \mid i \in I\right\}$ such that for $w_{j i}$ from above there exists a family $m_{i}: U_{i} \rightarrow Z_{\text {cont }}^{1}\left(G, \operatorname{Map}\left(G / N, L^{\infty}\left(\widehat{G} / N^{\perp}, U^{\mathrm{ab}}(\mathcal{H})\right)\right)\right)$ such that

$$
\begin{aligned}
\operatorname{Ad}\left(d_{*} w_{j i}(u)(h, z)(. .)\right)= & \operatorname{Ad}\left(\left(\delta_{g . .} \times \hat{g} . . m .\right)_{j i}(u)(h, z)(. .)\right) \\
& \in \mathrm{PL}^{\infty}\left(\widehat{G} / N^{\perp}, \mathrm{U}^{\mathrm{ab}}(\mathcal{H})\right)
\end{aligned}
$$

Proof : The proof uses a standard Zorn's lemma argument as it can be found in [Di].

First we note that the space $Z:=Z_{\text {cont }}^{1}\left(G, \operatorname{Map}\left(G / N, L^{\infty}\left(\widehat{G} / N^{\perp}, U^{\mathrm{ab}}(\mathcal{H})\right)\right)\right)$ is contractible. In fact, if $H:[0,1] \times \mathrm{U}^{\mathrm{ab}}(\mathcal{H}) \rightarrow \mathrm{U}^{\mathrm{ab}}(\mathcal{H})$ is a contraction with each $H\left(t_{-}\right)$a group homomorphism, then the push-forward $H_{\#}:[0,1] \times Z \rightarrow$ $Z$ preserves the cocycle relation and is a contraction.

We can assume that the atlas is sufficiently refined such that, firstly, $\varphi_{\ldots}$ from (27) is a boundary, i.e. there exist $\chi_{j i}: U_{j i} \rightarrow Z_{\text {cont }}^{1}(G, \operatorname{Map}(G / N, U(1)))$ such that $\left(\delta_{g} \chi\right)_{k j i}=\varphi_{k j i}$, and secondly, as $B$ is a paracompact Hausdorff space, even the closed cover $\bigcup_{i \in I} \bar{U}_{i}=B$ is locally finite and that $w_{j i}, \chi_{j i}$ are well-defined on $\bar{U}_{j i}$, for all $j, i \in I$. We let

$$
\begin{aligned}
& M:=\left\{\left(J, m_{.}\right) \mid J \subset I \text {, for all } j \in J: m_{j}: \bar{U}_{j} \rightarrow Z\right. \text { such that } \\
& \text { for all } i, j \in J \text { and all } u \in U_{j i}, z \in G / N \text { we have } \\
& m_{i}(u)(h, z)(. .)=d_{*} w_{j i}(u)(h)(z)(. .)^{-1} \chi_{j i}(u)(h, z) \\
& \left.m_{j}(u)\left(h, g_{j i}(u)+z\right)\left(\hat{g}_{j i}(u)+. .\right)\right\}
\end{aligned}
$$

For each $i \in I,(\{i\},\{\mathbb{1}\}) \in M$, because we can assume that $w_{i i}=\mathbb{1}, \chi_{i i}=1$, so $M$ is non-empty. We define a partial order on $M$ by $(J, m) \leq.\left(J^{\prime}, m_{\text {. }}\right)$ if and only if $J \subset J^{\prime} \subset I$ and $m_{j}=m_{j}^{\prime}$, for all $j \in J$. For each chain in $M$ the union of the index and cocycle sets is an upper bound, hence by Zorn's lemma there exists a maximal element $(J, m$.). Assume $J \neq I$, so there is some $a \in I \backslash J$. Let $R:=\bigcup_{j \in J}\left(\bar{U}_{j} \cap \bar{U}_{a}\right) \subset \bar{U}_{a}$. For $u \in R$ we define $\tilde{m}_{a}(u)(h, z)(.):.=$ $d_{*} w_{j a}(u)(h, z)(. .)^{-1} m_{j}(u)\left(h, g_{j a}(u)+z\right)\left(\hat{g}_{j a}(u)+..\right) \chi_{j a}(u)(h, z)$, if $u \in \bar{U}_{j}$. Due to $\delta_{g} \chi=\delta_{g \times \hat{g}} d_{*} w$, this definition is independent of $j \in J$. We end up with a diagram

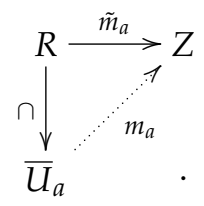

Since our cover is locally finite, $R$ is closed, but $Z$ is contractible, therefore an extension $m_{a}$ exists [DD, Lem. 4]. This contradicts the maximality of $\left(J, m_{.}\right)$, so $J=I$. Finally, equation (31) holds, since $\chi_{j i}(u)(h, z) \in \mathrm{U}(1)$.

The constructed family $\left\{m_{i}\right\}$ is the last ingredient to write down an explicit formula for a decker $\rho^{\text {dyn }}$ on (a stabilisation of) the pair $(P, E)$. It is not hard to 
guess that the cocycles $m_{i}$ will be an essential part of the cocycles $\mu_{i}^{\text {dyn }}$ which we will define to implement the decker $\rho^{\text {dyn }}$ locally. But unfortunately the constructed family $\left\{m_{i}\right\}$ is by no means unique, and this non-uniqueness is the origin of the following discussion. As a matter of fact this discussion will simplify drastically when we consider the special case of $G=\mathbb{R}^{n}$ with lattice $N=\mathbb{Z}^{n}$ in the next section below. However, now we continue with the discussion of almost strict triples from above and work out the general framework.

Let $\varphi_{. . .}$be the twisted Čech cocycle from equation (27). The triple under consideration is assumed to be almost strict, so (after refining the atlas $U_{\bullet}$ ) we have $\varphi_{k j i}=\left(\delta_{g} \chi_{. .}\right)_{k j i}$ for a chain $\chi_{. .} \in \check{C}^{1}\left(U_{\bullet}, Z_{\text {cont }}^{1}\left(G, \operatorname{Map}(G / N, \mathrm{U}(1)), g_{. .}\right)\right.$as in the proof above. Obviously, this chain $\chi$.. is only well defined up to a cocycle $\chi_{. .}^{1} \in \check{Z}^{1}\left(U_{\bullet}, Z_{\text {cont }}^{1}\left(G, \operatorname{Map}(G / N, U(1)), g_{. . .}\right)\right.$.

A choice of $\chi$.. determines the family $\left\{m_{i}\right\}$ in equation (32) not completely but up to a family $n_{i}: U_{i} \rightarrow Z_{\text {cont }}^{1}\left(G, \operatorname{Map}\left(G / N, L^{\infty}\left(\widehat{G} / N^{\perp}, \mathrm{U}^{\text {ab }}(\mathcal{H})\right)\right)\right)$ which satisfies $\left(\delta_{g \times \hat{g}} n \text {. }\right)_{j i}=\mathbb{1}$. We already mentioned that $m_{i}$ will be part of the cocycles $\mu_{i}^{\text {dyn }}$. Then it will turn out that the two families $\left\{m_{i}\right\}$ and $\left\{m_{i} n_{i}\right\}$ define exterior equivalent deckers. However, this is not the case when make we another choice of of $\chi_{. .}$, say $\chi_{. .} \chi_{. .}^{1}$ for $\chi_{. .}^{1}$ as above. Then $\left\{m_{i}\right\}$ must be replaced by $\left\{m_{i} m_{i}^{1}\right\}$ for $m_{i}^{1}$ being such that $\left(\delta_{g \times \hat{g}} m^{1}\right)_{j i}=\chi_{j i}^{1} \cdot \mathbb{1}$, and we will find that the corresponding deckers are exterior equivalent if and only if the class $\left[\chi_{. .}^{1}\right] \in \breve{H}^{1}\left(B, Z_{\text {cont }}^{1}(G / N, \operatorname{Map}(G / N, U(1))), g ..\right)$ vanishes. In other words, for each class $\left[\chi_{.}^{1}\right]$ we obtain a different class of dynamical triples. It is then the obvious question, whether the different dynamical triples still have something in common. Or if $x$ is the class of almost strict topological triple and $x^{\text {dyn }}$ is the class of one of the possible dynamical triples indicated, is there any relation between $x$ and $\tau(B)\left(x^{\text {dyn }}\right)$ ? Can we describe the difference, in particuar, when do they equal? A partial answer of this question is already given in Lemma 3.5 which shows that $x$ and $\tau(B)\left(x^{\mathrm{dyn}}\right)$ only can equal if $x$ is strict. 
Consider the short exact sequences

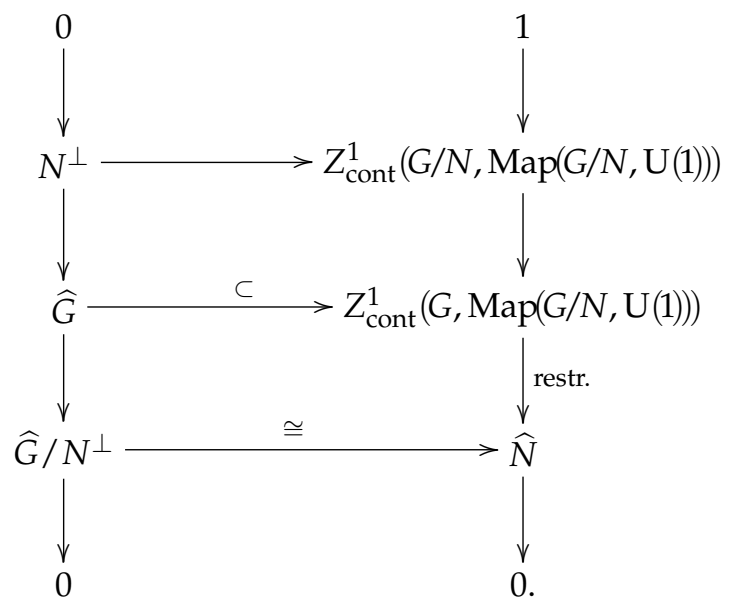

They both induce long exact sequences in (twisted) Čech cohomology. The relevant part for us is

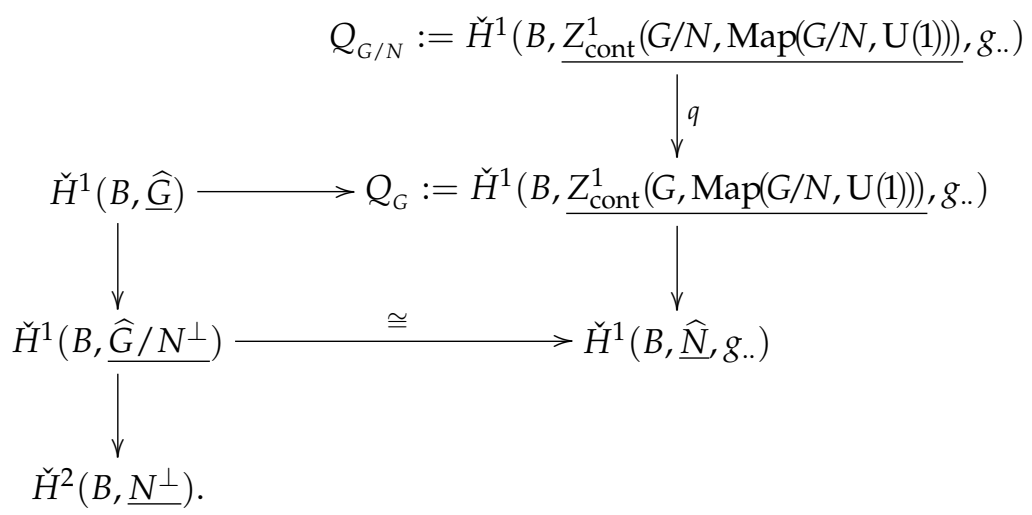

Due to equation (30) restriction $\left.\chi_{j i}(u)\right|_{N}$ defines a class $\left[\chi . . \mid{ }_{N}\right] \in \check{H}^{1}\left(B, \widehat{\widehat{N}}, g_{\text {.. }}\right)$ and by diagram (33) a class in $\breve{H}^{1}\left(B, \widehat{G} / N^{\perp}\right)$, i.e the class of a $\widehat{G} / N^{\perp}$-principal fibre bundle $\widehat{E}_{\chi}$.. $\rightarrow B$. Exactness of the columns in diagram (33) implies that this class is only well-defined up to the quotient group $Q_{G} / q\left(Q_{G / N}\right)$, because the class varies with the choice of $\chi$.. The bundle $\widehat{E}_{\chi \text {.. }}$ will be connected to the description of $\tau(B)\left(x^{\text {dyn }}\right)$. Namely, if $x=[(\kappa,(P, E),(\widehat{P}, \widehat{E}))]$ and $x^{\text {dyn }}=$ $\left[\left(\rho^{\text {dyn }}, P_{\text {dyn }}, E\right)\right]\left(P_{\text {dyn }}\right.$ will be a stabilisation of $P$ with a certain Hilbert space), then the result will be

$$
\tau(B)\left(x^{\mathrm{dyn}}\right)=\left[\left(\kappa^{\mathrm{top}},\left(P_{\text {dyn }_{\text {top }}} E\right),\left(\widehat{P_{\text {dyn }}}, \widehat{E} \oplus \widehat{E}_{\chi .}\right)\right)\right] .
$$

If the topological triple is strict, then by definition we can choose $\chi_{\text {.. }}$ such that $\left.\chi_{j i}(u)\right|_{N}=1$, so the class $\left[\left.\chi_{. .}\right|_{N}\right]$ vanishes and the bundle $\widehat{E}_{\chi . .}$ is trivialisable. In 
that case we give a description of $\widehat{P_{\mathrm{dyn}}}$ in the proof of the theorem below. If the topological triple is in the image of $\tau(B)$, then we even know more as the next theorem makes precise. Therein $\mathcal{P}(M)$ denotes the power set of a set $M$, and we denote the image of $\tau(B)$ by $\operatorname{Top}^{\mathrm{im}}(B)$, so

$$
\operatorname{Top}^{\mathrm{im}}(B) \subset \operatorname{Top}^{\mathrm{s}}(B) \subset \operatorname{Top}^{\text {as }}(B) \subset \operatorname{Top}(B) .
$$

Theorem 3.4 The map $\tau(B)$ is injective, and there are three maps

$$
\begin{aligned}
\delta^{\text {as }}(B) & : \operatorname{Top}^{\text {as }}(B) \rightarrow \mathcal{P}\left(\operatorname{Dyn}^{\dagger}(B)\right) \\
\delta^{\mathrm{s}}(B) & : \operatorname{Top}^{\mathrm{s}}(B) \rightarrow \mathcal{P}\left(\operatorname{Dyn}^{\dagger}(B)\right) \\
\delta^{\operatorname{im}}(B) & : \operatorname{Top}^{\operatorname{im}}(B) \rightarrow \operatorname{Dyn}^{\dagger}(B)
\end{aligned}
$$

with the following properties:

(a) For each $x \in \operatorname{Top}^{\text {as }}(B)$ the set $\delta^{\text {as }}(B)(x) \subset \operatorname{Dyn}^{\dagger}(B)$ is a $Q_{G}$-torsor.

(b) If $x \in \operatorname{Top}^{\mathrm{s}}(B)$, then $\delta^{\mathrm{s}}(B)(x) \subset \delta^{\mathrm{as}}(B)(x)$ is a $q\left(Q_{G / N}\right)$-subtorsor, and for each $x^{\text {dyn }} \in \delta^{\mathrm{s}}(B)(x)$ the (class of the) $\widehat{G} / N^{\perp}$-bundle $\widehat{E_{\text {dyn }}}$ of the (class of the) dual pair of $\tau(B)\left(x^{\text {dyn }}\right)$ is (the class of) the $\widehat{G} / N^{\perp}$-bundle $\widehat{E}$ of $x$.

(c) If $x$ is in the image of $\tau(B)$, then $\delta^{\mathrm{im}}(B)(x) \in \delta^{\mathrm{s}}(B)(x)$, and $\delta^{\mathrm{im}}(B)$ is the inverse of $\tau(B)$.

Moreover, $\delta^{?}(B)$ is natural in the sense that it extends to a natural transformation of functors

$$
\delta^{?}: \operatorname{Top}^{?} \rightarrow \mathcal{P} \circ \mathrm{Dyn}^{\dagger}, \quad ?=\text { as, s, im. }
$$

Proof : The proof consists of several steps which are rather technical, so we first give an overview of the proof:

In Step 1 we construct from the data of an almost strict topological triple a decker on the underlying pair (after stabilisation). The local construction will depend on many choices and it is the statement of Step 2 that almost all of these choices do not interfere with the equivalence class of triple defined. An exception is the choice of $\chi$.. (above) which will cause the $Q_{G}$-torsor and the $q\left(Q_{G / N}\right)$-subtorsor structure in $(a),(b)$. Then in Step 3 we compute the composition $\delta^{\mathrm{im}}(B) \circ \tau(B)$ and find that this is the identity on $\operatorname{Dyn}^{\dagger}(B)$, hence $\tau(B)$ is injective and $\delta^{\mathrm{im}}(B)$ is surjective. In Step 4 we compute the reverse composition $\tau(B) \circ \delta^{\mathrm{s}}(B)$, which will lead us to the result stated in $(b)$. In particular this calculation shows that $\tau(B) \circ \delta^{\mathrm{im}}(B)$ is the identity on the image of $\tau(B)$. As both compositions $\delta^{\mathrm{im}}(B) \circ \tau(B)$ and $\tau(B) \circ \delta^{\mathrm{im}}$ are the identity maps the statement of $(C)$ is clear then. In Step 5 we finally comment on the naturality of the maps.

In the hole of the proof we maintain the notation introduced above. 
Step 1: For an almost strict topological triple $(\kappa,(P, E),(\widehat{P}, \widehat{E}))$ we construct a dualisable dynamical triple $\left(\rho, P_{\mathrm{dyn}}, E_{\mathrm{dyn}}\right)$.

The idea is simple. All we have to do is to write down an explicit formula for a family of cocycles which satisfies equation (9) for the transition functions of the pair $(P, E)$.

Assume the topological triple has underlying Hilbert space $\widehat{\mathcal{H}}=L^{2}(G / N) \otimes$ $\mathcal{H}$. Let $\zeta_{j i}, \zeta_{j i}^{\prime}, v_{i}, w_{j i}, \varphi_{k j i}, \ldots$ be as above. We can assume that the atlas $U_{\bullet}$ is sufficiently refined such that $\varphi_{. . .}$is a boundary. So we choose $\chi_{\text {.. as above such }}$ that $\varphi_{\ldots .}=\delta_{g} \chi_{. .}$, then let $m_{i}$ be defined by (32) in the last lemma.

Let us consider $U_{j i} \ni u \mapsto \mathbb{1}_{\mathcal{H}} \otimes \lambda_{\widehat{G} / N^{\perp}}\left(\hat{g}_{j i}(u)\right) \in \mathrm{U}\left(\mathcal{H} \otimes L^{2}\left(\widehat{G} / N^{\perp}\right)\right)$, this defines a unitary implemented Čech cocycle, therefore it is a boundary, i.e. there are $l_{i}^{0}: U_{i} \rightarrow \mathrm{U}\left(\mathcal{H} \otimes L^{2}\left(\widehat{G} / N^{\perp}\right)\right)$ such that $l_{j}^{0}(u)^{-1} l_{i}^{0}(u)=\mathbb{1}_{\mathcal{H}} \otimes$ $\lambda_{\widehat{G} / N^{\perp}}\left(\hat{g}_{j i}(u)\right)$. Note that this need not be true for $\lambda_{\widehat{G} / N^{\perp}}\left(\hat{g}_{j i}(u)\right)$ alone as $\widehat{G} / N^{\perp}$ may be finite, $L^{2}\left(\widehat{G} / N^{\perp}\right)$ finite dimensional and $U\left(L^{2}\left(\widehat{G} / N^{\perp}\right)\right)$ not contractible. We conclude that there exists a family $\lambda_{i}: U_{i} \rightarrow \operatorname{Map}\left(G / N, \operatorname{PU}\left(\mathcal{H} \otimes L^{2}\left(\widehat{G} / N^{\perp}\right) \otimes\right.\right.$ $\left.\left.L^{2}(G / N) \otimes \mathcal{H}\right)\right)$ such that this family permits lifts $l_{i}: U_{i} \rightarrow \operatorname{Map}(G / N, \mathrm{U}(\mathcal{H} \otimes$ $\left.\left.L^{2}\left(\widehat{G} / N^{\perp}\right) \otimes L^{2}(G / N) \otimes \mathcal{H}\right)\right)$ such that

$$
\begin{aligned}
& l_{j}(u)\left(g_{j i}(u)+z\right)^{-1}\left(\mathbb{1} \otimes \mathbb{1} \otimes \bar{\zeta}_{j i}(u)(z)\right) l_{i}(u)(z) \\
= & \mathbb{1} \otimes \lambda_{\widehat{G} / N^{\perp}}\left(\hat{g}_{j i}(u)\right) \otimes \bar{\zeta}_{j i}(u)(z) .
\end{aligned}
$$

For example we may take $\lambda_{i}(u)(z)=\operatorname{Ad}\left(l_{i}^{0}(u) \otimes \mathbb{1} \otimes \mathbb{1}\right)$, but it will be important that we allow ourselves to have the freedom of a more flexible form of $\lambda_{i}$. One should also note that equation (34) is stated for unitary and not for projective unitary operators.

We define a family (of local isomorphisms ) $\vartheta_{i}: U_{i} \rightarrow \operatorname{Map}(G / N, \operatorname{PU}(\mathcal{H} \otimes$ $\left.\left.L^{2}\left(\widehat{G} / N^{\perp}\right) \otimes L^{2}(G / N) \otimes \mathcal{H}\right)\right)$ by

$$
\begin{aligned}
\vartheta_{i}(u)(z):= & \lambda_{i}(u)(z)\left(\mathbb{1}_{\mathcal{H}} \otimes \mathbb{1}_{L^{2}\left(\widehat{G} / N^{\perp}\right)} \otimes \kappa_{i}^{a}(u, z)\right) \\
& \operatorname{Ad}\left(\left(\mathbb{1}_{\mathcal{H}} \otimes \bar{\kappa}^{\sigma}(z, . .) \otimes \mathbb{1}_{\mathcal{H}}\right)\left(\mathbb{1}_{\mathcal{H}} \otimes v_{i}(u, z, . .)\right)\right)
\end{aligned}
$$

which we use to define another family (of cocycles)

$$
\mu_{i}^{\text {dyn }}: U_{i} \rightarrow Z_{\text {cont }}^{1}\left(G, \operatorname{Map}\left(G / N, \operatorname{PU}\left(\mathcal{H} \otimes L^{2}\left(\widehat{G} / N^{\perp}\right) \otimes L^{2}(G / N) \otimes \mathcal{H}\right)\right)\right.
$$

by

$$
\begin{aligned}
\mu_{i}^{\text {dyn }}(u)(h, z):= & \vartheta_{i}(u)(z+h N) \\
& \operatorname{Ad}\left(\left(m_{i}(u)(h, z) \otimes \mathbb{1}_{L^{2}(G / N)} \otimes \mathbb{1}_{\mathcal{H}}\right)\right. \\
& \left.\left(\mathbb{1}_{\mathcal{H}} \otimes\langle\hat{\sigma}(. .), h\rangle \otimes \mathbb{1}_{L^{2}(G / N)} \otimes \mathbb{1}_{\mathcal{H}}\right)\right) \\
& \vartheta_{i}(u)(z)^{-1} .
\end{aligned}
$$


The stabilised pair $\left(P_{\text {dyn }}, E_{\text {dyn }}\right):=\left(\mathrm{PU}\left(\mathcal{H} \otimes L^{2}\left(\widehat{G} / N^{\perp}\right)\right) \otimes P, E\right)$ has transition functions $g_{j i}, \zeta_{j i}^{\text {dyn }}:=\mathbb{1}_{\mathcal{H}} \otimes \mathbb{1}_{L^{2}\left(\widehat{G} / N^{\perp}\right)} \otimes \zeta_{j i}$, and we claim that on $\left(P_{\text {dyn }}, E_{\text {dyn }}\right)$ the family $\left\{\mu_{i}^{\text {dyn }}\right\}_{i \in I}$ defines a dualisable decker $\rho^{\text {dyn }}$. To verify this we have to check that

$$
\mu_{j}^{\mathrm{dyn}}(u)\left(h, g_{j i}(u)+z\right)^{-1} \zeta_{j i}^{\mathrm{dyn}}(u)(z+h N) \mu_{i}^{\mathrm{dyn}}(u)(h, z)=\zeta_{j i}^{\mathrm{dyn}}(u)(z) .
$$

Although lengthy, this is a straight forward calculation. Indeed, we have ${ }^{(1)}$

$$
\begin{aligned}
& \mu_{j}^{\mathrm{dyn}}\left(h, g_{j i}+z\right)^{-1} \zeta_{j i}^{\mathrm{dyn}}(z+h N) \mu_{i}^{\mathrm{dyn}}(h, z) \\
= & \vartheta_{j}\left(g_{j i}+z\right) \operatorname{Ad}\left(\left(m_{j}\left(h, g_{j i}+z\right)(. .)^{-1} \otimes \mathbb{1} \otimes \mathbb{1}\right)(\mathbb{1} \otimes\langle\hat{\sigma}(. .),-h\rangle \otimes \mathbb{1} \otimes \mathbb{1})\right) \\
& \vartheta_{j}\left(g_{j i}+z+h N\right)^{-1}\left(\mathbb{1} \otimes \mathbb{1} \otimes \zeta_{j i}(z+h N)\right) \vartheta_{i}(z+h N) \\
& \operatorname{Ad}\left(\left(m_{i}(h, z)(. .) \otimes \mathbb{1} \otimes \mathbb{1}\right)(\mathbb{1} \otimes\langle\hat{\sigma}(. .), h\rangle \otimes \mathbb{1} \otimes \mathbb{1})\right) \vartheta_{i}(z)^{-1} \\
= & \vartheta_{j}\left(g_{j i}+z\right) \operatorname{Ad}\left(\left(m_{j}\left(h, g_{j i}+z\right)(. .)^{-1} \otimes \mathbb{1} \otimes \mathbb{1}\right)(\mathbb{1} \otimes\langle\hat{\sigma}(. .),-h\rangle \otimes \mathbb{1} \otimes \mathbb{1})\right) \\
& \operatorname{Ad}\left(\left(\mathbb{1} \otimes v_{j}\left(g_{j i}+z+h N, . .\right)^{-1}\right)\left(\mathbb{1} \otimes \bar{\kappa}^{\sigma}\left(g_{j i}+z+h N, . .\right)^{-1} \otimes \mathbb{1}\right)\right) \\
& \left(\mathbb{1} \otimes \operatorname{Ad}\left(\lambda_{\widehat{G} / N^{\perp}}\left(\hat{g}_{j i}\right)\right) \otimes \zeta_{j i}^{\prime}(z+h N)\right) \\
& \operatorname{Ad}\left(\left(\mathbb{1} \otimes \bar{\kappa}^{\sigma}(z+h N, . .) \otimes \mathbb{1}\right)\left(\mathbb{1} \otimes v_{i}(z+h N, . .)\right)\right) \\
& \operatorname{Ad}\left(\left(m_{i}(h, z)(. .) \otimes \mathbb{1} \otimes \mathbb{1}\right)(\mathbb{1} \otimes\langle\hat{\sigma}(. .), h\rangle \otimes \mathbb{1} \otimes \mathbb{1})\right) \vartheta_{i}(z)^{-1} \\
= & \vartheta_{j}\left(g_{j i}+z\right) \operatorname{Ad}\left(\left(m_{j}\left(h, g_{j i}+z\right)(. .)^{-1} \otimes \mathbb{1} \otimes \mathbb{1}\right)(\mathbb{1} \otimes\langle\hat{\sigma}(. .),-h\rangle \otimes \mathbb{1} \otimes \mathbb{1})\right) \\
& \operatorname{Ad}\left(\mathbb{1} \otimes \lambda_{\widehat{G} / N^{\perp}}\left(g_{j i}\right) \otimes \mathbb{1} \otimes \mathbb{1}\right) \\
& \mathbb{1} \otimes\left[\operatorname{Ad}\left(v_{j}\left(g_{j i}+z+h N, \hat{g}_{j i}+. .\right)^{-1}\left(\bar{\kappa}^{\sigma}\left(z+h N, \hat{g}_{j i}+. .\right)^{-1} \otimes \mathbb{1}\right)\right)\right. \\
& \left.\left.\zeta_{j i}^{\prime}(z+h N)\right) \operatorname{Ad}\left(\left(\bar{\kappa}^{\sigma}(z+h N, . .) \otimes \mathbb{1}\right) v_{i}(z+h N, . .)\right)\right] \\
& \operatorname{Ad}\left(\left(m_{i}(h, z)(. .) \otimes \mathbb{1} \otimes \mathbb{1}\right)(\mathbb{1} \otimes\langle\hat{\sigma}(. .), h\rangle \otimes \mathbb{1} \otimes \mathbb{1})\right) \vartheta_{i}(z)^{-1} .
\end{aligned}
$$

The expression inside the squared brackets can be rewritten by equation (25). This reads

$$
\begin{aligned}
\ldots= & \vartheta_{j}\left(g_{j i}+z\right) \operatorname{Ad}\left(\left(m_{j}\left(h, g_{j i}+z\right)(. .)^{-1} \otimes \mathbb{1} \otimes \mathbb{1}\right)(\mathbb{1} \otimes\langle\hat{\sigma}(. .),-h\rangle \otimes \mathbb{1} \otimes \mathbb{1})\right) \\
& \operatorname{Ad}\left(\mathbb{1} \otimes \lambda_{\widehat{G} / N^{\perp}}\left(g_{j i}\right) \otimes \mathbb{1} \otimes \mathbb{1}\right) \\
& \mathbb{1} \otimes\left[\operatorname{Ad}\left({\overline{\zeta^{\prime}}}_{j i}(. .)\right)\left(A_{j i}(z+h N) \otimes \mathbb{1} \otimes \mathbb{1}\right)\right] \\
& \operatorname{Ad}\left(\left(m_{i}(h, z)(. .) \otimes \mathbb{1} \otimes \mathbb{1}\right)(\mathbb{1} \otimes\langle\hat{\sigma}(. .), h\rangle \otimes \mathbb{1} \otimes \mathbb{1})\right) \vartheta_{i}(z)^{-1} .
\end{aligned}
$$


If we insert furthermore the results of the previous lemmata, we obtain

$$
\begin{aligned}
\ldots= & \vartheta_{j}(z) \operatorname{Ad}\left(\left(m_{j}\left(h, g_{j i}+z\right)(. .)^{-1} \otimes \mathbb{1} \otimes \mathbb{1}\right)(\mathbb{1} \otimes\langle\hat{\sigma}(. .),-h\rangle \otimes \mathbb{1} \otimes \mathbb{1})\right) \\
& \operatorname{Ad}\left(\mathbb{1} \otimes \lambda_{\widehat{G} / N^{\perp}}\left(g_{j i}\right) \otimes \mathbb{1} \otimes \mathbb{1}\right) \\
& \operatorname{Ad}\left(\mathbb{1} \otimes{\overline{\hat{\zeta}^{\prime}}}_{j i}(. .)\right)\left(\mathbb{1} \otimes \beta_{j i}(z+h N) \otimes \mathbb{1} \otimes \mathbb{1}\right)\left(\operatorname{Ad}\left(w_{j i}(z+h N)(. .)\right) \otimes \mathbb{1} \otimes \mathbb{1}\right) \\
& \left(\gamma_{j i} \otimes \mathbb{1} \otimes \mathbb{1}\right) \operatorname{Ad}\left(\left(m_{i}(h, z)(. .) \otimes \mathbb{1} \otimes \mathbb{1}\right)(\mathbb{1} \otimes\langle\hat{\sigma}(. .), h\rangle \otimes \mathbb{1} \otimes \mathbb{1})\right) \vartheta_{i}(z)^{-1} \\
= & \vartheta_{j}(z) \operatorname{Ad}\left(\mathbb{1} \otimes \lambda_{\widehat{G} / N^{\perp}}\left(g_{j i}\right) \otimes \mathbb{1} \otimes \mathbb{1}\right) \\
& \operatorname{Ad}\left(\left(\mathbb{1} \otimes{\overline{\hat{\zeta}^{\prime}}}_{j i}(. .)\right)\left(\mathbb{1} \otimes\left\langle\hat{\sigma}\left(. .+\hat{g}_{j i}\right)-\hat{\sigma}(. .), \sigma\left(g_{j i}+z+h N\right)-h\right\rangle \otimes \mathbb{1} \otimes \mathbb{1}\right)\right. \\
& \left.\left(w_{j i}(z)(. .) \otimes \mathbb{1} \otimes \mathbb{1}\right)\right)\left(\gamma_{j i} \otimes \mathbb{1} \otimes \mathbb{1}\right) \vartheta_{i}(z)^{-1} .
\end{aligned}
$$

As $\sigma\left(g_{j i}+z+h N\right)-h$ and $\sigma\left(g_{j i}+z\right)$ differ by some element in $N$, we obtain the identity

$$
\begin{aligned}
& \left\langle\hat{\sigma}\left(. .+\hat{g}_{j i}\right)-\hat{\sigma}(. .), \sigma\left(g_{j i}+z+h N\right)-h\right\rangle \\
= & \left\langle\hat{\sigma}\left(. .+\hat{g}_{j i}\right)-\hat{\sigma}(. .), \sigma\left(g_{j i}+z+h N\right)-h-\sigma\left(g_{j i}+z\right)\right\rangle \\
& \left\langle\hat{\sigma}\left(. .+\hat{g}_{j i}\right)-\hat{\sigma}(. .), \sigma\left(g_{j i}+z\right)\right\rangle \\
= & \left\langle\hat{g}_{j i}(u), \sigma\left(g_{j i}+z+h N\right)-h-\sigma\left(g_{j i}+z\right)\right\rangle \\
& \left\langle\hat{\sigma}\left(. .+\hat{g}_{j i}\right)-\hat{\sigma}(. .), \sigma\left(g_{j i}+z\right)\right\rangle .
\end{aligned}
$$

Therein the first factor is a scalar and the second defines $\beta_{j i}$. So the main calculation continues

$$
\begin{aligned}
\ldots= & \vartheta_{j}(z) \operatorname{Ad}\left(\mathbb{1} \otimes \lambda_{\widehat{G} / N^{\perp}}\left(g_{j i}\right) \otimes \mathbb{1} \otimes \mathbb{1}\right) \operatorname{Ad}\left(\left(\mathbb{1} \otimes{\overline{\hat{\zeta}^{\prime}}}_{j i}(. .)\right)\right) \\
& \left(\mathbb{1} \otimes \beta_{j i}(z) \otimes \mathbb{1} \otimes \mathbb{1}\right)\left(\operatorname{Ad}\left(w_{j i}(z)(. .)\right) \otimes \mathbb{1} \otimes \mathbb{1}\right)\left(\gamma_{j i} \otimes \mathbb{1} \otimes \mathbb{1}\right) \vartheta_{i}(z)^{-1},
\end{aligned}
$$

and again by equation (25)

$$
\begin{aligned}
\ldots= & \vartheta_{j}(z) \operatorname{Ad}\left(\mathbb{1} \otimes \lambda_{\widehat{G} / N^{\perp}}\left(g_{j i}\right) \otimes \mathbb{1} \otimes \mathbb{1}\right) \\
& \mathbb{1} \otimes\left[\operatorname{Ad}\left(v_{j}\left(g_{j i}+z, \hat{g}_{j i}+. .\right)^{-1}\left(\bar{\kappa}^{\sigma}\left(z, \hat{g}_{j i}+. .\right)^{-1} \otimes \mathbb{1}\right)\right)\right. \\
& \left.\left(\mathbb{1} \otimes \zeta_{j i}^{\prime}(z)\right) \operatorname{Ad}\left(\left(\bar{\kappa}^{\sigma}(z, . .) \otimes \mathbb{1}\right) v_{i}(z, . .)\right)\right] \vartheta_{i}(z)^{-1} \\
= & \vartheta_{j}(z) \mathbb{1} \otimes \operatorname{Ad}\left(v_{j}\left(g_{j i}+z, . .\right)^{-1}\left(\bar{\kappa}^{\sigma}(z, . .)^{-1} \otimes \mathbb{1}\right)\right) \\
& \operatorname{Ad}\left(\mathbb{1} \otimes \lambda_{\widehat{G} / N^{\perp}}\left(g_{j i}\right)\right) \otimes \zeta_{j i}^{\prime}(z) \\
& \mathbb{1} \otimes \operatorname{Ad}\left(\left(\bar{\kappa}^{\sigma}(z, . .) \otimes \mathbb{1}\right) v_{i}(z, . .)\right) \vartheta_{i}(z)^{-1} \\
= & \mathbb{1} \otimes \mathbb{1} \otimes \zeta_{j i}(z) \\
= & \zeta_{j i}^{\text {dyn }}(z) .
\end{aligned}
$$

This shows that the $\mu_{i}^{\text {dyn }}$ s define a decker $\rho^{\text {dyn }}$ on $\left(P_{\text {dyn }}, E_{\text {dyn }}\right)$. By construction $\rho^{\text {dyn }}$ is dualisable, because $(h, z) \mapsto\left(m_{i}(u)(h, z)(..) \otimes \mathbb{1} \otimes \mathbb{1}\right)(\mathbb{1} \otimes\langle\hat{\sigma}(.), h.\rangle \otimes$ 
$\mathbb{1} \otimes \mathbb{1})$ is a continuous and unitary implemented 1-cocycle.

Step 2: The construction of Step 1 defines a map $\delta^{\text {as }}(B): \operatorname{Top}^{\text {as }}(B) \rightarrow \mathcal{P}\left(\operatorname{Dyn}^{\dagger}(B)\right)$ and $\delta^{\text {as }}(B)(x)$ is a $Q_{G}$-torsor, for each $x \in \operatorname{Top}^{\text {as }}(B)$. If $x$ is strict then there is a distinguished $q\left(Q_{G / N}\right)$-subtorsor $X^{\mathrm{dyn}}$, and if $x$ is in the image of $\tau(B)$ we single out a specific element $x^{0} \in X^{\text {dyn }}$. We then just define $\delta^{\mathrm{s}}(B)(x):=X^{\text {dyn }}$ and $\delta^{\mathrm{im}}(B)(x):=x^{0}$ in the respective cases.

We have to show that all choices involved do not change the class of the dynamical triple $\left(\rho^{\mathrm{dyn}}, P_{\mathrm{dyn}}, E_{\mathrm{dyn}}\right)$. That the choice of the atlas has no effect on the class of the constructed dynamical triple is rather obvious. It is less obvious for the choices of $\lambda_{i}$ (or $\left.l_{i}\right), m_{i}$ and the homotopy class of $\kappa$, i.e. the choice of an isomorphic topological triple. We convince ourselves that three other choices of these define exterior equivalent deckers.

Firstly, if we choose another $\lambda_{i}$, say $\lambda_{i}^{\prime}=\operatorname{Ad}\left(l_{i}^{\prime} \otimes \mathbb{1} \otimes \mathbb{1}\right)$, then by equation (34) the family $v_{i}(u)(z):=\lambda_{i}(u)(z) \lambda_{i}^{\prime-1}(u)(z)$ defines an automorphism of $P_{\text {dyn, }}$ and the Čech class of this automorphism $[v.] \in \check{H}^{1}(B, \underline{\mathrm{U}(1)})$ vanishes. Thus we are precisely in the situation of Example 2.1.

Secondly, equation (32) shows that $m_{i}$ is unique up to functions $n_{i}: U_{i} \rightarrow$ $Z_{\text {cont }}^{1}\left(G, \operatorname{Map}\left(G / N, L^{\infty}\left(\widehat{G} / N^{\perp}, \mathrm{U}^{\mathrm{ab}}(\mathcal{H})\right)\right)\right)$ such that $\delta_{g \times \hat{g}} n$. $=\mathbb{1}$. We change the atlas of the the constructed dynamical triple such that we have transition functions $\zeta_{j i}^{1}(u)(z):=\vartheta_{j}(u)\left(g_{j i}(u)+z\right)^{-1} \zeta_{j i}(u)(z) \vartheta_{i}(u)(z)$ and cocycles $\mu_{i}^{1}(u)(h, z):=\operatorname{Ad}\left(\left(m_{i}(u)(h, z) \otimes \mathbb{1}_{L^{2}(G / N)} \otimes \mathbb{1}_{\mathcal{H}}\right)\left(\mathbb{1}_{\mathcal{H}} \otimes\langle\hat{\sigma}(.), h.\rangle \otimes \mathbb{1}_{L^{2}(G / N)} \otimes\right.\right.$ $\left.\left.\mathbb{1}_{\mathcal{H}}\right)\right)$. Assume $m_{i}$ is changed by $n_{i}$. Then because of the commutativity of $\mathrm{U}^{\mathrm{ab}}(\mathcal{H})$ we have $n_{i}(u)(h+g, z)=\mu_{i}^{1}(u)(g, z)^{-1}\left(n_{i}(u)(h, z+g N)\right) n_{i}(u)(g, z)$; and because of the $\lambda_{i}$-terms in $\vartheta_{i}$ we have that $\zeta_{j i}^{1}(u)(z)\left(n_{i}(u)(h, z)(.).\right)=$ $n_{i}(u)(h, z)\left(. .-\hat{g}_{j i}(u)\right)=n_{j}(u)\left(h, g_{j i}(u)+z\right)(.$.$) . So c_{i}:=n_{i}$ defines an exterior equivalence (Definition 2.5).

Thirdly, if $\kappa^{\prime}$ is homotopic to $\kappa$, the Čech class of the bundle automorphism $\kappa^{\prime} \circ \mathcal{K}^{-1}$ vanishes, and the change of $\mu_{i}^{\mathrm{dyn}}$ caused by this automorphism is again covered by Example 2.1.

Let us now discuss the choice of $\chi_{\ldots}$. We always have the freedom to change it by a cocycle $\chi^{1} \in \check{Z}^{1}\left(U_{\bullet}, Z_{\text {cont }}^{1}(G, \operatorname{Map}(G / N, U(1))), g\right.$.. $)$. The consequence is a change of $m_{i}$ by $m_{i}^{1}: U_{i} \rightarrow Z_{\text {cont }}^{1}\left(G, \operatorname{Map}\left(G / N, L^{\infty}\left(\widehat{G} / N^{\perp}, U^{\mathrm{ab}}(\mathcal{H})\right)\right)\right)$ such that $\delta_{g \times \hat{g}} m_{.}^{1}=\chi_{. .}^{1}$. It is clear that if $\chi_{. .}^{1}=\delta_{g} \chi^{2}$ is a boundary, then the deckers $\rho^{\mathrm{dyn}}$ and $\rho^{\mathrm{dyn}}{ }^{1}$ which correspond to $m_{i}$ and $m_{i} m_{i}^{1}$ are exterior equivalent, for we can define $n_{i}:=\chi_{i}^{2} m_{i}^{1}$, so $\delta_{g \times \hat{g}} n$. $=\mathbb{1}$ which leads us to the case we already discussed above. If the class $\left[\chi_{. .}^{1}\right]$ does not vanish, then it follows that the corresponding deckers are not exterior equivalent and the corresponding triples are not stably outer conjugate.

Thus it follows that for each $\left[\chi_{. .}^{1}\right] \in Q_{G}$ we get a different dynamical triple, and we define $\delta^{\text {as }}(B)(x) \subset \operatorname{Dyn}^{\dagger}(B)$ to be the set of these dynamical triples. 
It is obvious that $Q_{G}$ acts freely and transitively on $\delta^{\text {as }}(B)(x)$, i.e. it is a $Q_{G}$ torsor. If the triple $x$ is strict, then we can choose $\chi$.. such that $\left.\chi_{j i}(u)\right|_{N}=1$, and this property is preserved by the action of $q\left(Q_{G / N}\right) \subset Q_{G}$. So we singled out a specific $q\left(Q_{G / N}\right)$-subtorsor $X^{\text {dyn }} \subset \delta^{\text {as }}(B)(x)$, and we define $\delta^{\mathrm{s}}(B)(x):=X^{\mathrm{dyn}}$. If $x$ is in the image of $\tau(B)$, then we saw in in the proof of Lemma 3.5 that $w_{j i}$ satisfies $d_{*} w_{j i}(u)(z) \in \mathrm{U}(1) \cdot \mathbb{1}$, so it is meaningful to define $\chi_{j i}:=d_{*} w_{j i}$. Then we let $x^{0} \in X^{\text {dyn }}$ be the element which corresponds to this particular choice of $\chi_{. .}$, and we put $\delta^{\operatorname{im}}(B)(x):=x^{0}$.

Step 3: $\delta^{\operatorname{im}}(B) \circ \tau(B)=\mathrm{id}_{\operatorname{Dyn}^{\dagger}(B)}$, so $\tau(B)$ is injective and $\delta(B)$ is surjective.

The formal calculation is similar to to what we did in the of the proof of Theorem 3.2.

Let $(\rho, P, E)$ be a dualisable dynamical triple having transition functions $g_{j i}, \zeta_{j i}$ and cocycles $\mu_{i}$. Recall the definition of $\tau(B)$ in particular of the topological triple $\left(\kappa^{\text {top }},\left(P_{\text {top }}, E\right),(\widehat{P}, \widehat{E})\right)$ out of which we must compute $\left(\rho^{\text {dyn }}, P_{\text {top }_{\text {dyn }}} E\right)$.

$P_{\text {top }_{\text {dyn }}}=\operatorname{PU}\left(\mathcal{H} \otimes L^{2}\left(\widehat{G} / N^{\perp}\right) \otimes L^{2}(G / N)\right) \otimes P$ is stably isomorphic to $P$ and we shall compute $\mu_{i}^{\text {dyn }}$. We first give one possible, explicit formula for $\lambda_{i}(u)(z)$. Let $\hat{\mathcal{F}}: L^{2}\left(\widehat{G} / N^{\perp}\right) \rightarrow L^{2}(N)$ be the inverse Fourier transform, then

$$
\hat{\mathcal{F}} \circ \lambda_{\widehat{G} / N^{\perp}}\left(\hat{g}_{j i}(u)\right) \circ \hat{\mathcal{F}}^{-1}=\left\langle\hat{g}_{j i}(u), .\right\rangle^{-1} \in L^{\infty}(N, \mathrm{U}(1)),
$$

and the definition of $\hat{g}_{j i}$ implies

$$
\begin{aligned}
& \operatorname{Ad}\left(\lambda_{\widehat{G} / N^{\perp}}\left(\hat{g}_{j i}(u)\right)\right) \otimes \zeta_{j i}^{\text {top }}(u)(z) \\
= & \operatorname{Ad}\left(\left.\left(\hat{\mathcal{F}}^{-1} \otimes \mathbb{1} \otimes \mathbb{1}\right) \circ \bar{\mu}_{j}(u)\left(., g_{j i}(u)+z\right)^{-1}\right|_{N} \circ(\hat{\mathcal{F}} \otimes \mathbb{1} \otimes \mathbb{1})\right) \\
& \mathbb{1} \otimes \zeta_{j i}^{\text {top }}(u)(z) \operatorname{Ad}\left(\left.\left(\hat{\mathcal{F}}^{-1} \otimes \mathbb{1} \otimes \mathbb{1}\right) \circ \bar{\mu}_{i}(u)(., z)\right|_{N} \circ(\hat{\mathcal{F}} \otimes \mathbb{1} \otimes \mathbb{1})\right) .
\end{aligned}
$$

Therefore we have

$$
\begin{aligned}
l_{i}(u)(z)= & \left(\left(\mathbb{1} \otimes \hat{\mathcal{F}}^{-1} \otimes \mathbb{1} \otimes \mathbb{1}\right) \circ\left(\left.\mathbb{1} \otimes \bar{\mu}_{i}(u)(., z)\right|_{N}\right) \circ(\mathbb{1} \otimes \hat{\mathcal{F}} \otimes \mathbb{1} \otimes \mathbb{1})\right. \\
& \in \mathrm{U}\left(\mathcal{H} \otimes L^{2}\left(\widehat{G} / N^{\perp}\right) \otimes L^{2}(G / N) \otimes \mathcal{H}\right)
\end{aligned}
$$

which is continuous by Lemma $3.1(i)$, hence $\lambda_{i}(u)(z)=\operatorname{Ad}\left(l_{i}(u)(z)\right)$. From the local definition of $\kappa^{\text {top }}$ in equation (21) we read off that

$$
\kappa_{i}(u)(z, \hat{z})=\kappa_{i}^{a}(u, z) \kappa^{\sigma}(z, \hat{z}) \operatorname{Ad}\left(v_{i}(u, z, \hat{z})\right)
$$

for $\kappa_{i}^{a}(u, z):=\operatorname{Ad}\left(\bar{\mu}_{i}(u)(-\sigma(-), z)^{-1}\right)$ and $v_{i}(u, z, \hat{z}):=\lambda_{G / N}(z) \otimes \mathbb{1}$. In the proof of Lemma 3.5 we already observed that in the case of a topological triple constructed out of dynamical one we have $d_{*} w_{j i}(u)(z) \in \mathrm{U}(1) \cdot \mathbb{1}$, and by the definition of $\delta^{\mathrm{im}}(B)$ we choose $\chi_{\text {.. }}:=d_{*} w_{. .}$. Therefore we can choose $m_{i}=$ $\mathbb{1}$. As a consequence the first $\mathcal{H}$-slot of the tensor product $\mathcal{H} \otimes L^{2}\left(\widehat{G} / N^{\perp}\right) \otimes$ 
$L^{2}(G / N) \otimes \mathcal{H}$ will contain the identity operator $\mathbb{1}$ only. We have all ingredients together to compute $\mu_{i}^{\text {dyn }(i)}$.

$$
\begin{aligned}
\mu_{i}^{\mathrm{dyn}}(h, z)= & \vartheta_{i}(z+h N)(\mathbb{1} \otimes\langle\hat{\sigma}(. .), h\rangle \otimes \mathbb{1} \otimes \mathbb{1}) \vartheta_{i}(z)^{-1} \\
= & \operatorname{Ad}\left(\left.\left(\mathbb{1} \otimes \hat{\mathcal{F}}^{-1} \otimes \mathbb{1} \otimes \mathbb{1}\right) \circ \mathbb{1} \otimes \bar{\mu}_{i}(., z+h N)\right|_{N} \circ(\mathbb{1} \otimes \hat{\mathcal{F}} \otimes \mathbb{1} \otimes \mathbb{1})\right. \\
& \left(\mathbb{1} \otimes \mathbb{1} \otimes \bar{\mu}_{i}(-\sigma(-), z+h N)^{-1}\right) \\
& \left.\left.\left(\mathbb{1} \otimes \bar{\kappa}^{\sigma}(z+h N, . .)\right)\left(\mathbb{1} \otimes \mathbb{1} \otimes \lambda_{G / N}(z+h N) \otimes \mathbb{1}\right)\right)\right) \\
& (\mathbb{1} \otimes\langle\hat{\sigma}(. .), h\rangle \otimes \mathbb{1} \otimes \mathbb{1}) \\
& \left.\left(\mathbb{1} \otimes \mathbb{1} \otimes \lambda_{G / N}(-z) \otimes \mathbb{1}\right)\left(\mathbb{1} \otimes \bar{\kappa}^{\sigma}(z+h N, . .)^{-1}\right)\right) \\
& \left(\mathbb{1} \otimes \mathbb{1} \otimes \bar{\mu}_{i}(-\sigma(-), z)\right) \\
& \left.\left(\mathbb{1} \otimes \hat{\mathcal{F}}^{-1} \otimes \mathbb{1} \otimes \mathbb{1}\right) \circ \mathbb{1} \otimes\left(\left.\bar{\mu}_{i}(., z)\right|_{N}\right)^{-1} \circ(\mathbb{1} \otimes \hat{\mathcal{F}} \otimes \mathbb{1} \otimes \mathbb{1})\right) .
\end{aligned}
$$

Since $(\hat{\mathcal{F}} \otimes \mathbb{1}) \circ\left\langle\hat{\sigma}(.),. h-\sigma\left({ }_{-}+h N\right)+\sigma\left({ }_{-}\right)\right\rangle \circ\left(\hat{\mathcal{F}}^{-1} \otimes \mathbb{1}\right)=\lambda_{N}\left(h-\sigma\left({ }_{-}+h N\right)+\right.$ $\sigma(-)) \in \mathrm{U}\left(L^{2}\left(\widehat{G} / N^{\perp}\right) \otimes L^{2}(G / N)\right)$ and by the cocycle identity for $\bar{\mu}_{i}$ this transforms to

$$
\begin{aligned}
\ldots= & \operatorname{Ad}\left(\left(\mathbb{1} \otimes \hat{\mathcal{F}}^{-1} \otimes \mathbb{1} \otimes \mathbb{1}\right) \circ\left(\mathbb{1} \otimes \bar{\mu}_{i}\left(\sigma\left(_{-}\right)+., z+h N-_{-}\right)\right)\right. \\
& \left.\left(\mathbb{1} \otimes \lambda_{G / N}(h N) \otimes \mathbb{1} \otimes \mathbb{1}\right)\left(\mathbb{1} \otimes \lambda_{N}\left(h-\sigma(-+h N)+\sigma\left({ }_{-}\right)\right)\right) \otimes \mathbb{1}\right) \\
& \left.\left(\mathbb{1} \otimes\left(\bar{\mu}_{i}\left(\sigma(-)+., z-_{-}\right)\right)^{-1}\right) \circ(\mathbb{1} \otimes \hat{\mathcal{F}} \otimes \mathbb{1} \otimes \mathbb{1})\right) \\
= & \operatorname{Ad}\left(\left(\mathbb{1} \otimes \hat{\mathcal{F}}^{-1} \otimes \mathbb{1} \otimes \mathbb{1}\right) \circ\right. \\
& \left.\left(\mathbb{1} \otimes \lambda_{G / N}(h N) \otimes \mathbb{1} \otimes \mathbb{1}\right)\left(\mathbb{1} \otimes \lambda_{N}\left(h-\sigma\left({ }_{-}+h N\right)+\sigma\left({ }_{-}\right)\right)\right) \otimes \mathbb{1}\right) \\
& \left(\mathbb{1} \otimes \bar{\mu}_{i}\left(\sigma(-+h N)+.+h-\sigma\left({ }_{-}+h N\right)+\sigma(-), z-_{-}\right)\right) \\
& \left(\mathbb{1} \otimes\left(\bar{\mu}_{i}(-\sigma(-)-., z)\right)\right) \\
& \circ(\mathbb{1} \otimes \hat{\mathcal{F}} \otimes \mathbb{1} \otimes \mathbb{1})) \\
= & \operatorname{Ad}\left(\left(\mathbb{1} \otimes \hat{\mathcal{F}}^{-1} \otimes \mathbb{1} \otimes \mathbb{1}\right) \circ\right. \\
& \left.\left(\mathbb{1} \otimes \lambda_{G / N}(h N) \otimes \mathbb{1} \otimes \mathbb{1}\right)\left(\mathbb{1} \otimes \lambda_{N}(h-\sigma(-+h N)+\sigma(-))\right) \otimes \mathbb{1}\right) \\
& \left.\left(\mathbb{1} \otimes \mathbb{1} \otimes \mathbb{1} \otimes\left(\bar{\mu}_{i}(h, z)\right)\right) \circ(\mathbb{1} \otimes \hat{\mathcal{F}} \otimes \mathbb{1} \otimes \mathbb{1})\right) .
\end{aligned}
$$

Let $\mathcal{S}: L^{2}(N) \otimes L^{2}(G / N) \rightarrow L^{2}(G)$ be the isomorphism introduced on page 47 . There we discussed its behaviour with respect to the left regular representation on $L^{2}(G)$. This leads us finally to

$$
\begin{aligned}
\mu_{i}^{\mathrm{dyn}}(u)(h, z)= & \operatorname{Ad}\left(\left(\mathbb{1} \otimes \hat{\mathcal{F}}^{-1} \otimes \mathbb{1} \otimes \mathbb{1}\right) \circ\left(\mathbb{1} \otimes \mathcal{S}^{-1} \otimes \mathbb{1}\right) \circ\right. \\
& \left.\left(\mathbb{1} \otimes \lambda_{G}(h) \otimes \bar{\mu}_{i}(u)(h, z)\right)\right) \\
& \circ(\mathbb{1} \otimes \mathcal{S} \otimes \mathbb{1}) \circ(\mathbb{1} \otimes \hat{\mathcal{F}} \otimes \mathbb{1} \otimes \mathbb{1})) .
\end{aligned}
$$


Thus we have shown that $(\rho, P, E)$ and $\left(\rho^{\mathrm{dyn}}, P_{\mathrm{top}_{\mathrm{dyn}}}, E\right)$ are outer conjugate.

Step 4: We compute $\left.\tau(B)\left(\delta^{\mathrm{s}}\right)(B)(x)\right)$ which will complete the statement of $(b)$. In particular this calculation shows that $\tau(B) \circ \delta^{\mathrm{im}}(B)=\mathrm{id}_{\mathrm{im}(\tau(B))}$.

Let $(\kappa,(P, E),(\widehat{P}, \widehat{E}))$ be a topological triple with underlying Hilbert space $\widehat{\mathcal{H}}=$ $L^{2}(G / N) \otimes \mathcal{H}$. For the first steps we only need the assumption that the triple is almost strict. So let $\left(\rho^{\mathrm{dyn}}, P_{\mathrm{dyn}}, E_{\mathrm{dyn}}\right)$ be the dynamical triple which we constructed in Step 1. As we saw $E_{\text {dyn }}$ is nothing but $E$ itself. From this dynamical triple we are going to construct the topological triple

$$
\left(\kappa^{\mathrm{top}},\left(P_{\mathrm{dyn}_{\mathrm{top}}}, E_{\mathrm{dyn}}\right),\left(\widehat{P_{\mathrm{dyn}}}, \widehat{E_{\mathrm{dyn}}}\right)\right)
$$

(according to the construction of $\tau(B)$ ) which we then have to compare with $(\kappa,(P, E),(\widehat{P}, \widehat{E}))$.

To compute the dual pair $\left(\widehat{P_{\mathrm{dyn}}}, \widehat{E_{\mathrm{dyn}}}\right)$ we with determination of $\phi_{j i}^{\mathrm{dyn}}$ the second term of the total cocycle $\left(\psi_{. .}^{\text {dyn }}, \phi_{. .}^{\text {dyn }}, 1\right)$ of the constructed dynamical triple $\left(\rho^{\mathrm{dyn}}, P_{\mathrm{dyn}}, E_{\mathrm{dyn}}\right)$. By equation (16), the restriction of $\phi_{j i}^{\mathrm{dyn}}$ to $N$ defines the (the cocycle of) bundle $\widehat{E_{\text {dyn }}}$.

The definition of the cocycles $\mu_{i}^{\text {dyn }}$ of the decker $\rho^{\text {dyn }}$ contains $\lambda_{i}$. We make use of the possibility to choose $\lambda_{i}(u)(z)=\operatorname{Ad}\left(l_{i}^{0}(u) \otimes \mathbb{1} \otimes \mathbb{1}\right)$ as we explained in Step 1. In Step 2 we saw that this choice does not have any effect on the level of equivalence classes. Assume that the atlas is sufficiently refined and consists of contractible charts such that the following continuous lifts exist. Lifts of the transition functions $\bar{\zeta}_{j i}^{\text {dyn }}=\mathbb{1} \otimes \mathbb{1} \otimes \bar{\zeta}_{j i}$ and lifts $\bar{\kappa}_{i}^{a}: U_{i} \rightarrow L^{\infty}(G / N, \mathrm{U}(\widehat{\mathcal{H}}))$ of $\kappa^{a}$ which we use to define unitary lifts $\bar{\mu}_{i}^{\text {dyn }}$ of $\mu_{i}^{\text {dyn }}$ according to (35) in the obvious way, i.e. we drop the Ad. Let $\delta_{j i}: U_{j i} \rightarrow L^{\infty}(G / N, \mathrm{U}(1))$ be such that $\alpha_{j i}(u)(z, \hat{z})=\delta_{j i}(u)(z)\left\langle\hat{\sigma}\left(\hat{g}_{j i}(u)+\hat{z}\right)-\hat{\sigma}(\hat{z}), \sigma\left(g_{j i}(u)+z\right\rangle w_{j i}(u)(z)(\hat{z}) \bar{\gamma}_{j i}(u)\right.$, for a lift $\bar{\gamma}_{j i}: U_{j i} \rightarrow \mathrm{U}(\mathcal{H})$ of $\gamma_{j i}$; therein all notation is as above. Then by definition

$$
\begin{aligned}
& \phi_{j i}^{\mathrm{dyn}}(u)(h, z) \\
= & \bar{\mu}_{i}^{\mathrm{dyn}}(u)(h, z) \bar{\zeta}_{j i}^{\mathrm{dyn}}(u)(z)^{-1} \bar{\mu}_{j}^{\mathrm{dyn}}(u)\left(h, g_{j i}(u)+z\right)^{-1} \bar{\zeta}_{j i}^{\mathrm{dyn}}(u)(z+h N),
\end{aligned}
$$

and if we repeat the calculation of Step 1 on the unitary level, there are four equalities which must be modified by $\mathrm{U}(1)$-valued functions. Namely, equation (36) by $\delta(u)(z+h N)$, equation (37) by $\chi_{j i}$ from equation (32), equation (39) by the scalar term of (38) and equation $(40)$ by $\delta(u)(z)^{-1}$. We finally find

$$
\begin{aligned}
\phi_{j i}^{\mathrm{dyn}}(u)(h, z)= & \left\langle\hat{g}_{j i}(u), \sigma\left(g_{j i}(u)+z+h N\right)-h-\sigma\left(g_{j i}(u)+z\right)\right\rangle \\
& \chi_{j i}(u)(h, z)\left(d \delta_{j i}(u)\right)(h, z) .
\end{aligned}
$$


It follows that $\widehat{E_{\mathrm{dyn}}} \cong \widehat{E}$ if and only if the topological triple we started with is strict and we have chosen $\chi_{.}$such that $\left.\chi_{j i}(u)\right|_{N}=1$. Indeed, the cocycles of these bundles are $\hat{g}_{j i}^{\mathrm{dyn}}(u):=\left(\left.\phi_{j i}^{\mathrm{dyn}}(u)(., z)\right|_{N}\right)^{-1}=\left.\hat{g}_{j i}(u) \chi_{j i}(u)(-., z)\right|_{N}$. This proves $(b)$.

To complete the computation of the dual we have to compute

$\widehat{\zeta_{j i}^{\text {dyn }}}: U_{j i} \rightarrow \operatorname{Map}\left(\widehat{G} / N^{\perp}, \operatorname{PU}\left(L^{2}(G / N) \otimes \mathcal{H} \otimes L^{2}\left(\widehat{G} / N^{\perp}\right) \otimes L^{2}(G / N) \otimes \mathcal{H}\right)\right)$.

by equation (18). The reader should not be confused by the two different $L^{2}(G / N)$ factors in the tensor product. The first is due to the stabilisation in the definition of the dual (18), and the second is due to the Hilbert space we started with which is $L^{2}(G / N) \otimes \mathcal{H}$. We use the symbols $\smile$ and _ to distinguish multiplication operators on the two Hilbert spaces; $\smile$ for the first factor, due to the definition of the dual and _ for the second factor as we did all the time. The dual transition functions are given by ${ }^{\circledR}$

$$
\begin{aligned}
\widehat{\zeta_{j i}^{\mathrm{dyn}}}(\hat{z})= & \operatorname{Ad}\left(\left(\overline{\mathcal{\kappa}}^{\sigma}\left(-g_{j i}, \hat{g}_{j i}^{\mathrm{dyn}}+\hat{z}\right) \otimes \mathbb{1} \otimes \mathbb{1} \otimes \mathbb{1} \otimes \mathbb{1}\right)\right. \\
& \left(\lambda_{G / N}\left(-g_{j i}\right) \otimes \mathbb{1} \otimes \mathbb{1} \otimes \mathbb{1} \otimes \mathbb{1}\right) \\
& \left.\left(\bar{\zeta}_{j i}^{\mathrm{dyn}}(-\smile)\right)\left(\phi_{j i}^{\mathrm{dyn}}(-\sigma(\smile), 0)^{-1} \otimes \mathbb{1} \otimes \mathbb{1} \otimes \mathbb{1} \otimes \mathbb{1}\right)\right) \\
= & \operatorname{Ad}\left(\left(\bar{\kappa}^{\sigma}\left(-g_{j i}, \hat{g}_{j i}^{\mathrm{dyn}}+\hat{z}\right) \otimes \mathbb{1} \otimes \mathbb{1} \otimes \mathbb{1} \otimes \mathbb{1}\right)\right. \\
& \left(\lambda_{G / N}\left(-g_{j i}\right) \otimes \mathbb{1} \otimes \mathbb{1} \otimes \mathbb{1} \otimes \mathbb{1}\right) \\
& \left(\mathbb{1} \otimes l_{j}^{0} \otimes \mathbb{1} \otimes \mathbb{1}\right)\left[\mathbb{1} \otimes \lambda_{\widehat{G} / N^{\perp}}\left(\hat{g}_{j i}\right) \otimes\left(\bar{\zeta}_{j i}(-\smile)\right]\right. \\
& \left.\left(\mathbb{1} \otimes l_{i}^{0^{-1}} \otimes \mathbb{1} \otimes \mathbb{1}\right)\left(\phi_{j i}^{\mathrm{dyn}}(-\sigma(\smile), 0)^{-1} \otimes \mathbb{1} \otimes \mathbb{1} \otimes \mathbb{1} \otimes \mathbb{1}\right)\right) .
\end{aligned}
$$

By equation (25) this is ${ }^{(1)}$

$$
\begin{aligned}
\ldots= & \operatorname{Ad}\left(\left(\mathbb{1} \otimes l_{j}^{0} \otimes \mathbb{1} \otimes \mathbb{1}\right)\right. \\
& \left(\bar{\kappa}^{\sigma}\left(-g_{j i}, \hat{g}_{j i}^{\mathrm{dyn}}+\hat{z}\right) \otimes \mathbb{1} \otimes \mathbb{1} \otimes \mathbb{1} \otimes \mathbb{1}\right)\left(\lambda_{G / N}\left(-g_{j i}\right) \otimes \mathbb{1} \otimes \mathbb{1} \otimes \mathbb{1} \otimes \mathbb{1}\right) \\
& \left(\mathbb{1} \otimes \mathbb{1} \otimes \bar{\kappa}_{j}^{a}\left(g_{j i}-\smile\right)\right)\left(\mathbb{1} \otimes \mathbb{1} \otimes \bar{\kappa}^{\sigma}\left(g_{j i}-\smile, \hat{g}_{j i}+\hat{z}\right) \otimes \mathbb{1}\right) \\
& \left(\mathbb{1} \otimes \mathbb{1} \otimes v_{j}\left(g_{j i}-\smile, \hat{g}_{j i}+\hat{z}\right)\right)\left(\mathbb{1} \otimes \mathbb{1} \otimes \bar{\kappa}_{j}^{b}\left(\hat{g}_{j i}+\hat{z}\right)\right) \\
& \left(\mathbb{1} \otimes \mathbb{1} \otimes \lambda_{\widehat{G}^{\prime} N^{\perp}}\left(\hat{g}_{j i}\right) \otimes \overline{\hat{\zeta}}_{j i}(\hat{z})\right)\left(\alpha_{j i}(-\smile, \hat{z}) \otimes \mathbb{1} \otimes \mathbb{1} \otimes \mathbb{1} \otimes \mathbb{1}\right) \\
& \left(\mathbb{1} \otimes \mathbb{1} \otimes \bar{\kappa}_{i}^{b}(\hat{z})^{-1}\right)\left(\mathbb{1} \otimes \mathbb{1} \otimes v_{i}(-\smile, \hat{z})^{-1}\right) \\
& \left(\mathbb{1} \otimes \mathbb{1} \otimes \bar{\kappa}^{\sigma}(-\smile, \hat{z})^{-1} \otimes \mathbb{1}\right)\left(\mathbb{1} \otimes \mathbb{1} \otimes \bar{\kappa}_{i}^{a}(-\smile)^{-1}\right) \\
& \left(\phi_{j i}^{\mathrm{dyn}}(-\sigma(\smile), 0)^{-1} \otimes \mathbb{1} \otimes \mathbb{1} \otimes \mathbb{1} \otimes \mathbb{1}\right) \\
& \left.\left(\mathbb{1} \otimes l_{i}^{0^{-1}} \otimes \mathbb{1} \otimes \mathbb{1}\right)\right),
\end{aligned}
$$


and if we insert the formulas for $\bar{\kappa}^{\sigma}, \alpha_{j i}$ and $\phi_{j i}^{\mathrm{dyn}}$ from above, we end up with ${ }^{\natural}$

$$
\begin{aligned}
\ldots= & \operatorname{Ad}\left(\left(\mathbb{1} \otimes l_{j}^{0} \otimes \mathbb{1} \otimes \mathbb{1}\right)\right. \\
& \left(\left\langle\hat{\sigma}\left(\hat{g}_{j i}^{\mathrm{dyn}}+\hat{z}\right), \sigma\left(\smile+g_{j i}\right)-\sigma(\smile)\right\rangle \otimes \mathbb{1} \otimes \mathbb{1} \otimes \mathbb{1} \otimes \mathbb{1}\right) \\
& \left(\lambda_{G / N}\left(-g_{j i}\right) \otimes \mathbb{1} \otimes \mathbb{1} \otimes \mathbb{1} \otimes \mathbb{1}\right) \\
& \left(\mathbb{1} \otimes \mathbb{1} \otimes \bar{\kappa}_{j}^{a}\left(g_{j i}-\smile\right)\right) \\
& \left(\mathbb{1} \otimes \mathbb{1} \otimes\left\langle\hat{\sigma}\left(\hat{g}_{j i}+\hat{z}\right), \sigma\left(--g_{j i}+\smile\right)-\sigma(-)\right\rangle \otimes \mathbb{1}\right) \\
& \left(\mathbb{1} \otimes \mathbb{1} \otimes v_{j}\left(g_{j i}-\smile, \hat{g}_{j i}+\hat{z}\right)\right)\left(\mathbb{1} \otimes \mathbb{1} \otimes \bar{\kappa}_{j}^{b}\left(\hat{g}_{j i}+\hat{z}\right)\right) \\
& \left(\mathbb{1} \otimes \mathbb{1} \otimes \lambda_{\widehat{G} / \mathrm{N}^{\perp}}\left(\hat{g}_{j i}\right) \otimes \overline{\hat{\zeta}}_{j i}(\hat{z})\right)\left(\delta_{j i}(-\smile) \otimes \mathbb{1} \otimes \mathbb{1} \otimes \mathbb{1} \otimes \mathbb{1}\right) \\
& \left(\left\langle\hat{\sigma}\left(\hat{g}_{j i}+\hat{z}\right)-\hat{\sigma}(\hat{z}), \sigma\left(g_{j i}-\smile\right)\right\rangle \otimes \mathbb{1} \otimes \mathbb{1} \otimes \mathbb{1} \otimes \mathbb{1}\right) \\
& \left(w_{j i}(-\smile)(\hat{z}) \otimes \mathbb{1} \otimes \mathbb{1} \otimes \mathbb{1}\right)\left(\mathbb{1} \otimes \bar{\gamma}_{j i} \otimes \mathbb{1} \otimes \mathbb{1} \otimes \mathbb{1}\right) \\
& \left(\mathbb{1} \otimes \mathbb{1} \otimes \bar{\kappa}_{i}^{b}(\hat{z})^{-1}\right)\left(\mathbb{1} \otimes \mathbb{1} \otimes v_{i}(-\smile, \hat{z})^{-1}\right) \\
& (\mathbb{1} \otimes \mathbb{1} \otimes\langle\hat{\sigma}(\hat{z}),-\sigma(-+\smile)+\sigma(-)\rangle \otimes \mathbb{1}) \\
& \left(\mathbb{1} \otimes \mathbb{1} \otimes \bar{\kappa}_{i}^{a}(-\smile)^{-1}\right) \\
& \left(\left[\left\langle\hat{g}_{j i}, \sigma\left(g_{j i}-\smile\right)+\sigma(\smile)-\sigma\left(g_{j i}\right)\right\rangle \chi_{j i}(-\sigma(\smile), 0)\right.\right. \\
& \left.\left.\left(d \delta_{j i}\right)(-\sigma(\smile), 0)\right]^{-1} \otimes \mathbb{1} \otimes \mathbb{1} \otimes \mathbb{1} \otimes \mathbb{1}\right) \\
& \left.\left(\mathbb{1} \otimes l_{i}^{0-1} \otimes \mathbb{1} \otimes \mathbb{1}\right)\right) .
\end{aligned}
$$

It follows from the definition of $\bar{\gamma}_{j i}$ that $\operatorname{Ad}\left(w_{j i}(u)(0)(\hat{z})^{-1}\right)=\gamma_{j i}(u)$, so after some further manipulation with all the $\langle\ldots\rangle$-expressions this finally transforms to ${ }^{(1)}$

$$
\begin{aligned}
\ldots= & \operatorname{Ad}\left(\left(\mathbb{1} \otimes l_{j}^{0} \otimes \mathbb{1} \otimes \mathbb{1}\right)\right. \\
& \left(\mathbb{1} \otimes \mathbb{1} \otimes \bar{\kappa}_{j}^{a}(-\smile)\right) \\
& \left(\mathbb{1} \otimes \mathbb{1} \otimes\left\langle\hat{\sigma}\left(\hat{g}_{j i}+\hat{z}\right),-\sigma(\smile)+\sigma\left(\_+\smile\right)-\sigma(-)\right\rangle \otimes \mathbb{1}\right) \\
& \left(\mathbb{1} \otimes \mathbb{1} \otimes v_{j}\left(-\smile, \hat{g}_{j i}+\hat{z}\right)\right)\left(\mathbb{1} \otimes \mathbb{1} \otimes \bar{\kappa}_{j}^{b}\left(\hat{g}_{j i}+\hat{z}\right)\right) \\
& \left.\lambda_{G / N}\left(-g_{j i}\right) \otimes \mathbb{1} \otimes \lambda_{\widehat{G} / N^{\perp}}\left(\hat{g}_{j i}\right) \otimes \overline{\hat{\zeta}}_{j i}(\hat{z})\right) \\
& \left(w_{j i}(-\smile)(\hat{z}) w_{j i}(0)(\hat{z})^{-1} \chi_{j i}(-\sigma(\smile), 0)^{-1} \otimes \mathbb{1} \otimes \mathbb{1} \otimes \mathbb{1}\right) \\
& \left(\mathbb{1} \otimes \mathbb{1} \otimes \bar{\kappa}_{i}^{b}(\hat{z})^{-1}\right)\left(\mathbb{1} \otimes \mathbb{1} \otimes v_{i}(-\smile, \hat{z})^{-1}\right) \\
& (\mathbb{1} \otimes \mathbb{1} \otimes\langle\hat{\sigma}(\hat{z}), \sigma(\smile)-\sigma(-+\smile)+\sigma(-)\rangle \otimes \mathbb{1}) \\
& \left(\mathbb{1} \otimes \mathbb{1} \otimes \bar{\kappa}_{i}^{a}(-\smile)^{-1}\right) \\
& \left(\mathbb{1} \otimes l_{i}^{0-1} \otimes \mathbb{1} \otimes \mathbb{1}\right) \\
& \left.\left(\left\langle\hat{\sigma}\left(\hat{g}_{j i}^{\text {dyn }}+\hat{z}\right)-\hat{\sigma}\left(\hat{g}_{j i}+\hat{z}\right),-\sigma\left(\smile-g_{j i}\right)+\sigma(\smile)\right\rangle \otimes \mathbb{1} \otimes \mathbb{1} \otimes \mathbb{1} \otimes \mathbb{1}\right)\right) .
\end{aligned}
$$

So far we did not use that the topological triple we started with is strict and 
that we want to compute the composition $\tau(B) \circ \delta^{\mathrm{s}}(B)$. We use this now, so the $G$-slot of $\chi_{j i}$ factors through $G / N$ and in particular $\hat{g}_{j i}^{\text {dyn }}=\hat{g}_{j i}$, so we find

$$
\ldots=\eta_{j}^{\prime}\left(u, \hat{g}_{j i}(u)+\hat{z}\right)\left(\xi_{j i}(u)(\hat{z}) \otimes \hat{\zeta}_{j i}(u)(\hat{z})\right) \eta_{i}^{\prime}(u)(z)^{-1}
$$

wherein we have used the short hands

$$
\begin{aligned}
\xi_{j i}(u)(\hat{z}):= & \operatorname{Ad}\left(\left[\left(\lambda_{G / N}\left(-g_{j i}(u)\right) \otimes \mathbb{1}\right) w_{j i}(u)(-\smile)(\hat{z}) w_{j i}(u)(0)(\hat{z})^{-1}\right.\right. \\
& \left.\left.\chi_{j i}(u)(-\sigma(\smile), 0)^{-1}\right] \otimes \lambda_{\widehat{G} / N^{\perp}}\left(\hat{g}_{j i}(u)\right)\right) \\
& \in \mathrm{U}\left(L^{2}(G / N) \otimes \mathcal{H} \otimes L^{2}\left(\widehat{G} / N^{\perp}\right)\right)
\end{aligned}
$$

and

$$
\begin{aligned}
\eta_{i}^{\prime}(u)(z):= & \operatorname{Ad}\left(\left(\mathbb{1} \otimes l_{i}^{0}(u) \otimes \mathbb{1} \otimes \mathbb{1}\right)\left(\mathbb{1} \otimes \mathbb{1} \otimes \bar{\kappa}_{i}^{a}(u)(-\smile)\right)\right. \\
& (\mathbb{1} \otimes \mathbb{1} \otimes\langle\hat{\sigma}(\hat{z}),-\sigma(\smile)+\sigma(-+\smile)-\sigma(-)\rangle \otimes \mathbb{1}) \\
& \left.\left(\mathbb{1} \otimes \mathbb{1} \otimes v_{i}(u,-\smile, \hat{z})\right)\left(\mathbb{1} \otimes \mathbb{1} \otimes \bar{\kappa}_{i}^{b}(u)(\hat{z})\right)\right) \\
& \in \operatorname{PU}\left(L^{2}(G / N) \otimes \mathcal{H} \otimes L^{2}\left(\widehat{G} / N^{\perp}\right) \otimes L^{2}(G / N) \otimes \mathcal{H}\right) .
\end{aligned}
$$

At this point we can read off that the bundle defined by $\xi_{j i}$ is trivialisable if $\chi_{. .}^{-1} d_{*} w_{j i}=\mathbb{1}$ which is the datum we must consider when we compute $\tau(B) \circ$ $\delta^{\mathrm{im}}(B)$ (cp. Step 2). In this case let $x_{i}: U_{i} \rightarrow \operatorname{Map}\left(\widehat{G} / N^{\perp}, \mathrm{U}\left(L^{2}(G / N) \otimes \mathcal{H} \otimes\right.\right.$ $\left.\left.L^{2}\left(\widehat{G} / N^{\perp}\right)\right)\right)$ be such that $\operatorname{Ad}\left(x_{j}(u)\left(\hat{g}_{j i}(u)+\hat{z}\right) x_{i}(u)(\hat{z})^{-1}\right)=\xi_{j i}(u)(\hat{z})$. Then we have shown that $\eta_{i}(u)(\hat{z}):=\eta_{i}^{\prime}(u)(\hat{z}) \operatorname{Ad}\left(x_{i}(u)(\hat{z}) \otimes \mathbb{1} \otimes \mathbb{1}\right)$ defines a family of local isomorphisms which fit together to a global isomorphism of principal bundles $\eta: \widehat{P_{\mathrm{s}}} \rightarrow \widehat{P_{\mathrm{dyn}}}$. The subscript s denotes stabilisation with respect to the Hilbert space $L^{2}(G / N) \otimes \mathcal{H} \otimes L^{2}\left(\widehat{G} / N^{\perp}\right)$. We claim that the topological triple $(\kappa,(P, E),(\widehat{P}, \widehat{E}))$ and the constructed triple $\left(\kappa^{\text {top }},\left(P_{\text {dyn }_{\text {top }}}, E\right),\left(\widehat{P_{\text {dyn }}}, \widehat{E}\right)\right)$ are equivalent; $\kappa^{\text {top }}$ is defined by equation (21) out of $\mu_{i}^{\text {dyn }}$. This will prove the identity $\tau(B) \circ \delta(B)=\mathrm{id}_{\mathrm{im}(\tau(B)}$. We show that the diagram

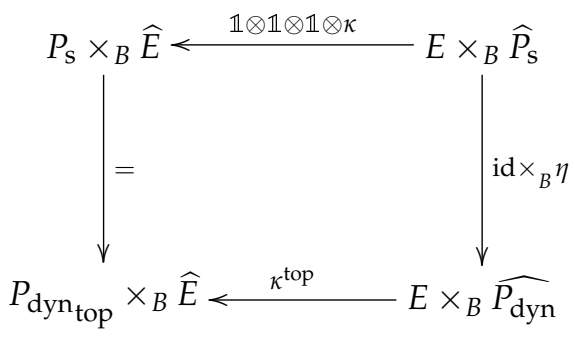

commutes up to homotpy. Locally, this means that there exist continuous maps $V_{i}: U_{i} \times G / N \times \widehat{G} / N^{\perp} \rightarrow \mathrm{PU}\left(L^{2}(G / N) \otimes \mathcal{H} \otimes L^{2}\left(\widehat{G} / N^{\perp}\right) \otimes L^{2}(G / N) \otimes \mathcal{H}\right)$ 
such that $\kappa_{i}^{\text {top }}(u)(z, \hat{z}) \eta_{i}(u)(\hat{z})=\left(\mathbb{1} \otimes \mathbb{1} \otimes \mathbb{1} \otimes \kappa_{i}(u)(z, \hat{z})\right) \operatorname{Ad}\left(V_{i}(u, z, \hat{z})\right)$. To prove this we will use that the projective unitary group is homotopy comutative in the sense of Corollary A.1. We have ${ }^{(1)}$

$$
\begin{aligned}
& \kappa_{i}^{\text {top }}(z, \hat{z}) \eta_{i}(\hat{z}) \\
= & \operatorname{Ad}((\langle\hat{\sigma}(\hat{z}), \sigma(\smile-z)-\sigma(\smile)\rangle \otimes \mathbb{1} \otimes \mathbb{1} \otimes \mathbb{1} \otimes \mathbb{1}) \\
& \left.\left(\mu_{i}^{\text {dyn }}(-\sigma(\smile), z)\right)^{-1}\left(\lambda_{G / N}(z) \otimes \mathbb{1} \otimes \mathbb{1} \otimes \mathbb{1} \otimes \mathbb{1}\right)\right) \eta_{i}(\hat{z}) \\
= & \operatorname{Ad}((\langle\hat{\sigma}(\hat{z}), \sigma(\smile-z)-\sigma(\smile)\rangle \otimes \mathbb{1} \otimes \mathbb{1} \otimes \mathbb{1} \otimes \mathbb{1}) \\
& \left(\mathbb{1} \otimes l_{i}^{0} \otimes \mathbb{1} \otimes \mathbb{1}\right)\left(\mathbb{1} \otimes \mathbb{1} \otimes \mathbb{1} \otimes \bar{\kappa}_{i}^{a}(z)\right) \\
& (\mathbb{1} \otimes \mathbb{1} \otimes\langle\hat{\sigma}(. .), \sigma(--z)-\sigma(-)\rangle \otimes \mathbb{1}) \\
& \left(\mathbb{1} \otimes \mathbb{1} \otimes v_{i}(z, . .)\right) \\
& (\mathbb{1} \otimes\langle\hat{\sigma}(. .), \sigma(\smile)\rangle \otimes \mathbb{1} \otimes \mathbb{1}) \\
& \left(m_{i}(-\sigma(\smile), z)(. .)^{-1} \otimes \mathbb{1} \otimes \mathbb{1}\right) \\
& \left(\mathbb{1} \otimes v_{i}(z-\smile, . .)^{-1}\right) \\
& (\mathbb{1} \otimes\langle\hat{\sigma}(. .),-\sigma(--z+\smile)+\sigma(-)\rangle \otimes \mathbb{1}) \\
& \left(\mathbb{1} \otimes \mathbb{1} \otimes \mathbb{1} \otimes \bar{\kappa}_{i}^{a}(z-\smile)^{-1}\right)\left(\mathbb{1} \otimes l_{i}^{0}-1\right. \\
& \left(\lambda_{G / N}(z) \otimes \mathbb{1} \otimes \mathbb{1}\right) \\
& \left(\mathbb{1} \otimes l_{i}^{0} \otimes \mathbb{1} \otimes \mathbb{1}\right)\left(\mathbb{1} \otimes \mathbb{1} \otimes \bar{\kappa}_{i}^{a}(-\smile)\right) \\
& (\mathbb{1} \otimes \mathbb{1} \otimes\langle\hat{\sigma}(\hat{z}),-\sigma(\smile)+\sigma(.+\smile)-\sigma(-)\rangle \otimes \mathbb{1}) \\
& \left(\mathbb{1} \otimes \mathbb{1} \otimes v_{i}(-\smile, \hat{z})\right)\left(\mathbb{1} \otimes \mathbb{1} \otimes \bar{\kappa}_{i}^{b}(\hat{z})\right) \\
& \left.\left(x_{i}(\hat{z}) \otimes \mathbb{1} \otimes \mathbb{1}\right)\right) .
\end{aligned}
$$

We see that the terms $\kappa_{i}^{a}(u)(z)$ and $\kappa_{i}^{b}(u)(z)$ occur. All terms with $l_{i}^{0}, m_{i}, v_{i}, x_{i}$ are continuous unitary maps The only bracket $\langle\ldots\rangle$ terms not continuous as unitary maps are the two terms in the first and third last line. If we collect them, we shall better pay attention to $\lambda_{G / N}(z)$ which acts in the $\smile$-variable. Then these two terms equal

$$
\begin{aligned}
& \left\langle\hat{\sigma}(\hat{z}), \sigma\left({ }_{-}+\smile-z\right)-\sigma\left(_{-}\right)-\sigma(\smile)\right\rangle \\
= & \langle\hat{\sigma}(\hat{z}), \sigma(--z)-\sigma(-)\rangle \\
& \left\langle\hat{\sigma}(\hat{z}),-\sigma(--z)+\sigma\left(_{-}+\smile-z\right)-\sigma(\smile)\right\rangle \\
= & \left(\mathbb{1} \otimes \bar{\kappa}^{\sigma}(z, \hat{z})\right)\left\langle\hat{z},-\sigma(--z)+\sigma\left(_{-}+\smile-z\right)-\sigma(\smile)\right\rangle \\
& \in \mathrm{U}\left(L^{2}(G / N) \otimes L^{2}(G / N)\right)
\end{aligned}
$$


and the second factor is continuous as a unitary map. Therefore we have

$$
\begin{aligned}
\kappa_{i}^{\text {top }}(u)(z, \hat{z}) \eta_{i}(u)(\hat{z})=\ldots=\quad & \left(\mathbb{1} \otimes \mathbb{1} \otimes \mathbb{1} \otimes \kappa_{i}^{a}(u)(z)\right) \\
& \left(\mathbb{1} \otimes \mathbb{1} \otimes \mathbb{1} \otimes \kappa^{\sigma}(z, \hat{z})\right) \\
& \left(\mathbb{1} \otimes \mathbb{1} \otimes \mathbb{1} \otimes \kappa_{i}^{b}(u)(z)\right) \operatorname{Ad}\left(V_{i}^{\prime}(u, z, \hat{z})\right) \\
& \left(\mathbb{1} \otimes \mathbb{1} \otimes \mathbb{1} \otimes \kappa_{i}(u)(z, \hat{z}) \operatorname{Ad}\left(V_{i}(u, z, \hat{z})\right),\right.
\end{aligned}
$$

for suitable continuous unitary maps $V_{i}^{\prime}, V_{i}$, and we are done.

Step 5: Naturality.

In the same way as we already stated for $\tau$, we have a commutative diagram

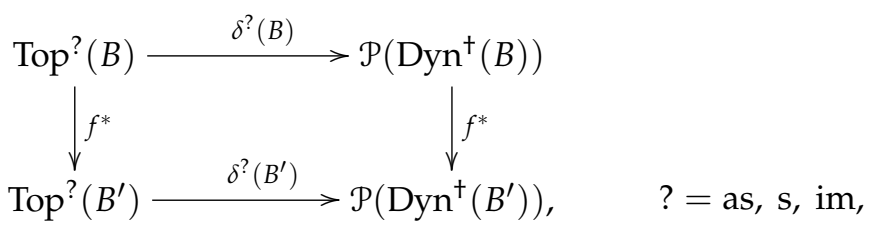

for each continuous map between base spaces $f: B^{\prime} \rightarrow B$

Definition 3.3 Let $P \rightarrow E \rightarrow B$ be a pair over the base space B. We say that this pair has an extension to a topological (resp. almost strict topological, strict topological, dynamical or dualisable dynamical) triple if the class $[(P, E)] \in \operatorname{Par}(B)$ is in the image of the forgetful map from topological (resp. almost strict topological, strict topological, dynamical or dualisable dynamical) triples over $B$ to pairs over $B$.

Corollary 3.1 Let $(P, E)$ be a pair over $B$. Then the following are equivalent:

(i) $(P, E)$ has an extension to a strict topological triple;

(ii) $(P, E)$ has an extension to an almost strict topological triple;

(iii) $(P, E)$ has an extension to a dualisable dynamical triple.

Proof : This is an immediate consequence of the previous theorem.

\subsection{The Case of $G=\mathbb{R}^{n}$ and $N=\mathbb{Z}^{n}$}

So far we kept our analysis completely general in the sense that we did not specify the groups $G, N$. We now turn to the important case of $G=\mathbb{R}^{n}$ with lattice $N=\mathbb{Z}^{n}, n=1,2,3, \ldots$ In the whole of this section we use the notation $\mathbb{T}^{n}:=\mathbb{R}^{n} / \mathbb{Z}^{n}$ for the torus and $\hat{\mathbb{T}}^{n}:=\widehat{\mathbb{R}}^{n} / \mathbb{Z}^{n \perp}$ for the dual torus. There should be no confusion to decide between $\hat{\mathbb{T}}^{n}$ and the dual group $\widehat{\mathbb{T}^{n}} \cong \mathbb{Z}^{n}$.

The first thing we should check is that in case of $G=\mathbb{R}^{n}, N=\mathbb{Z}^{n}$ our definition of topological triples agrees with the one introduced in [BRS]. 
The definition of T-duality triples in [BRS, Def. 2.8] differs in two points from what we stated in Definiton 2.9. The first point is that they use the language of twists [BRS, A.1] instead of $\mathrm{PU}(\mathcal{H})$-principal fibre bundles to model T-duality diagrams. But the category of $\mathrm{PU}(\mathcal{H})$-principal bundles with homotopy classes of bundle isomorphisms as morphisms is a model of twists, and our notion of stable equivalence of topological triples leads to the same equivalence classes as twists modulo isomorphism. This, because the notion of equivalence we use for topological triples requires the commutativity of diagram (14) only up to homotopy. To explain the second point let us consider the filtration of $H^{3}(E, \mathbb{Z})$ associated to the Leray-Serre spectral sequence

$$
0 \subset F^{3} H^{3}(E, \mathbb{Z}) \subset F^{2} H^{3}(E, \mathbb{Z}) \subset F^{1} H^{3}(E, \mathbb{Z}) \subset H^{3}(E, \mathbb{Z}) .
$$

By definition, an element $h \in H^{3}(E, \mathbb{Z})$ is in the subgroup $F^{k+1} H^{3}(E, \mathbb{Z})$ if for any $k$-dimensional $C W$-complex $C$ and any map $f: C \rightarrow B h$ is in the kernel of the induced map $f^{*}: H^{3}(E, \mathbb{Z}) \rightarrow H^{3}\left(C \times_{B} E, \mathbb{Z}\right)$. In our definition of a topological triple $(\kappa,(P, E),(\widehat{P}, \widehat{E}))$ we require that the class $[P] \in H^{3}(E, \mathbb{Z})$ of the bundle $P \rightarrow E$ lies in the subgroup $F^{1} H^{3}(E, \mathbb{Z})$ which is equivalent to the requirement of the triviality of $P$ over the fibres of $E \rightarrow B$ (see Definition 2.2); analogously for $\widehat{P}$. In [BRS] the definition of T-duality triples requires that the class $[P]$ is even in the second step of the filtration $[P] \in F^{2} H^{3}(E, \mathbb{Z})$; analogously for $\widehat{P}$. The following lemma states that in the case of $G=\mathbb{R}^{n}$ and $N=\mathbb{Z}^{n}$ these two conditions are equivalent.

Lemma 3.7 Let $G=\mathbb{R}^{n}$ and $N=\mathbb{Z}^{n}$, and let $(\kappa,(P, E),(\widehat{P}, \widehat{E}))$ be a topological triple over $B$, then

$$
[P] \in F^{2} H^{3}(E, \mathbb{Z}) \text { and }[\widehat{P}] \in F^{2} H^{3}(\widehat{E}, \mathbb{Z}) .
$$

Proof : Let $C$ be any 1-dimensional CW-complex and $f: C \rightarrow B$ any continuous map. We have to show that the class $[P]$ is in the kernel of the induced map $f^{*}: H^{3}(E, \mathbb{Z}) \rightarrow H^{3}\left(C \times{ }_{B} E, \mathbb{Z}\right)$. As $C$ is 1-dimensional, $H^{2}(C, \mathbb{Z})=0$ and there are no non-trivial torus bundles over it. Thus, if we pull back the topological triple $(\kappa,(P, E),(\widehat{P}, \widehat{E}))$ along $f$, it becomes

$$
\left(\kappa_{C},\left(P_{C}, C \times_{B} E \cong C \times \mathbb{T}^{n}\right),\left(\widehat{P}_{C}, C \times_{B} \widehat{E} \cong C \times \hat{\mathbb{T}}^{n}\right)\right)
$$

and the centre part of the corresponding diagram degenerates to the projections

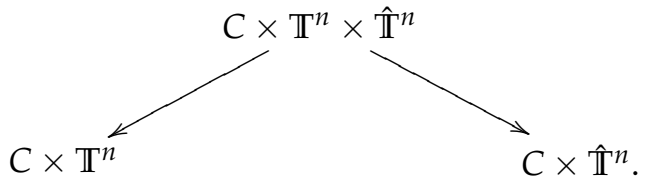


We extend this diagram to

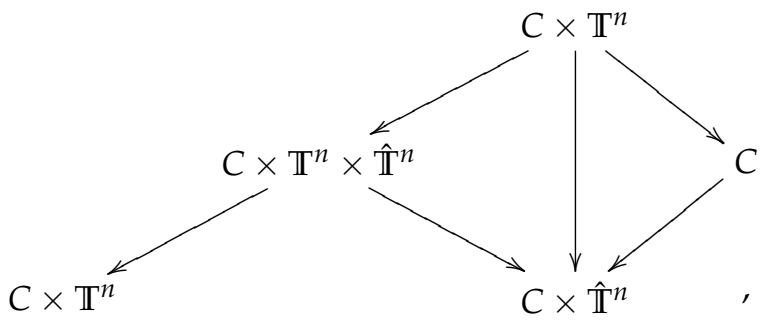

with the obvious inclusions and projection, so the diagonal composition is the identity. When we apply the the cohomology functor $H^{3}(., \mathbb{Z})$ to this diagram the vertical arrow becomes zero as it factors over $H^{2}(C, \mathbb{Z})=0$, but as $f^{*}[P]=$ $\left[P_{C}\right] \in H^{3}\left(C \times \mathbb{T}^{n}, \mathbb{Z}\right)$ is mapped by the identity from the left lower to the right upper group and as $\left[P_{C}\right]$ and $\left[\widehat{P}_{C}\right]$ equal when pulled back to $H^{3}(C \times$ $\mathbb{T}^{n} \times \hat{\mathbb{T}}^{n}, \mathbb{Z}$ ), we conclude it is zero, since its image in the right upper group coincides with the image of $f^{*}[\widehat{P}]=\left[\widehat{P}_{C}\right]$ under the vertical arrow.

This proves that $f^{*}[P]=0 \in H^{3}\left(C \times{ }_{B} E, \mathbb{Z}\right)$ and the same argument shows that the corresponding statement is true for $[\widehat{P}]$.

So we observe that our functor Top : \{base spaces $\} \rightarrow\{$ sets $\}$ is the same functor which is introduced in [BRS, Def. 2.11] under the name Triple $e_{n}$, and below we are going to use a central result of [BRS] about this functor, namely that the functor Top $=\operatorname{Triple}_{n}$ is representable by a space $R_{n}$ (see Lemma 3.9 below).

We now want to discuss Theorem 3.4 in the case of $G=\mathbb{R}^{n}, N=\mathbb{Z}^{n}$. In this case the question which of the topological triples are almost strict has a trivial answer and also the torsor structure of Theorem 3.4 becomes trivial. The criterion we make use of is the following lemma.

Lemma 3.8 Let $\mathrm{Z}$ be a topological abelian group which is contractible as topological space and is equipped with a continuous (right) G/N-action. Then

$$
\check{H}^{k}\left(B, \underline{Z},\left[g_{. .}\right]\right)=0 \quad k=1,2 .
$$

Proof : Similar to the proof of Lemma 3.6, the proof makes use of the standard Zorn's lemma argument.

$\mathrm{k}=1$ : Let $\left\{U_{i}\right\}_{i \in I}$ be an open cover of $B$, and let $\varphi_{\text {.. }} \in \check{Z}^{1}\left(U_{\bullet}, \underline{Z}\right)$ be a twisted 1-cocycle. We shall construct functions $\chi_{i}: U_{i} \rightarrow Z$ such that $\left(\delta_{g} \chi\right)$.. $=\varphi_{. . .} B$ is paracompact, hence without restriction we can assume that even the closed 
cover $\left\{\bar{U}_{i}\right\}$ is locally finite and all $\varphi_{i j}$ are defined on the whole of $\bar{U}_{i j}$. Let

$$
\begin{aligned}
K:=\{(J, \chi .) \quad \mid & J \subset I, \text { for all } j \in J: \chi_{j}: \bar{U}_{j} \rightarrow Z, \\
& \text { such that for all } j, k \in J \text { and } u \in \bar{U}_{j k}: \\
& \left.\chi_{j}(u) \chi_{k}(u)^{-1} \cdot g_{k j}(u)=\varphi_{k j}(u)\right\}
\end{aligned}
$$

$K$ is non-empty, since $\left(\{i\},\left\{\varphi_{i i}\right\}\right) \in L$, for each $i \in I$. We define a partial order on $K$ such that every chain has an upper bound. We let $(J, \chi) \leq.\left(J^{\prime}, \chi^{\prime}\right)$ if and only if $J \subset J^{\prime} \subset I$ and $\chi_{j}=\chi_{j}^{\prime}$, for all $j \in J$. By Zorn's lemma, let $(J, \chi$.) denote a maximal element of $K$.

Assume $J \neq I$, so there is some $a \in I \backslash J$. Let $R:=\bigcup_{j \in J}\left(\bar{U}_{j} \cap \bar{U}_{a}\right) \subset \bar{U}_{a}$. For $u \in R$ we define $\tilde{\chi}_{a}(u):=\varphi_{j a}(u) \chi_{j}(u) \cdot g_{j a}(u)$ if $u \in \bar{U}_{j}$. This definition is independent of $j \in J$. We end up with a diagram

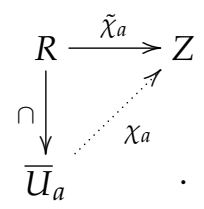

Since our cover is locally finite, $R$ is closed, but $Z$ is contractible, therefore an extension $\chi_{a}$ exists [DD, Lem. 4]. This contradicts the maximality of $(J, \chi$. $)$, so $J=I$.

$\mathrm{k}=2$ : Let $\varphi_{\ldots} \in \check{Z}^{2}\left(U_{\bullet}, \underline{Z}\right)$ be a twisted 2-cocycle. We shall construct functions $\chi_{i j}: U_{j i} \rightarrow Z$ such that $\left(\delta_{g} \chi\right)_{\ldots}=\varphi_{\ldots}$. Again by paracompactness of $B$ we can assume that even the closed cover $\left\{\bar{U}_{i}\right\}$ is locally finite and all $\varphi_{i j k}$ are defined on the whole of $\bar{U}_{i j k}$. Let

$$
\begin{aligned}
& L:=\left\{\left(J, \chi_{. .}\right) \quad \mid \quad J \subset I, \text { for all } i, j \in J: \chi_{i j}: \bar{U}_{i j} \rightarrow Z,\right. \\
& \text { such that for all } i, j, k \in J \text { and } u \in \bar{U}_{i j k}: \\
&\left.\chi_{j i}(u) \chi_{k i}(u)^{-1} \chi_{k j}(u) \cdot g_{j i}(u)=\varphi_{k j i}(u)\right\}
\end{aligned}
$$

The set $L$ is non-empty, since for each $i \in I\left(\{i\},\left\{\varphi_{i i i}\right\}\right) \in L$. We define a partial order on $L$ such that every chain has an upper bound. We let $\left(J, \chi_{. .}\right) \leq\left(J^{\prime}, \chi_{. .}^{\prime}\right)$ if and only if $J \subset J^{\prime} \subset I$ and $\chi_{i j}=\chi_{i j}^{\prime}$, for all $i, j \in J$. By Zorn's lemma, let $\left(J, \chi_{\text {.. }}\right)$ denote a maximal element of $L$. Assume $a \in I \backslash J$. Then let

$$
\begin{aligned}
M_{a}:= & \left\{\left(K, \psi_{\cdot a}\right) \mid K \subset J, \text { for all } k \in K: \psi_{k a}: \bar{U}_{k a} \rightarrow Z,\right. \\
& \text { such that for all } k, l \in K \text { and for } u \in \bar{U}_{l k a} \\
& \left.\psi_{k a}(u) \psi_{l a}(u)^{-1} \chi_{k l}(u) \cdot g_{l a}(u)=\varphi_{l k a}(u)\right\}
\end{aligned}
$$


The set $M_{a}$ is non-empty as for each $j \in J$ we find $(\{j\},\{1\}) \in M_{a}$. This because we always have $1 \cdot 1 \cdot \chi_{j j}(u) \cdot g_{j a}(u)=\varphi_{j j j}(u) \cdot g_{j a}(u)=\varphi_{j j a}(u)$. In the same manner as before, let $\left(K, \psi_{\cdot a}\right) \leq\left(K^{\prime}, \psi_{. a}^{\prime}\right)$ if and only if $K \subset K^{\prime} \subset I$ and $\psi_{k a}=\psi_{k a}^{\prime}$, for all $k \in K$. $\leq$ is a partial order on $M_{a}$ and every chain has an upper bound. Let $(K, \psi . a)$ be a maximal element. Assume $b \in J \backslash K$. Let $S:=$ $\bigcup_{k \in K}\left(\bar{U}_{k} \cap \bar{U}_{b} \cap \bar{U}_{a}\right) \subset \bar{U}_{b a}$. For $u \in R$ we define $\tilde{\psi}_{b a}(u):=\varphi_{k b a}(u) \chi_{k b}(u)$. $g_{b a}(u)^{-1} \psi_{k a}(u)$, if $u \in \bar{U}_{k b a}, k \in K$. By a one line calculation we find that this definition is independent of $k \in K$. So we have a diagram

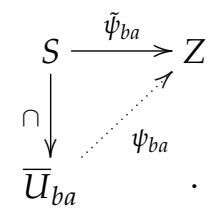

$S$ is closed, since our cover is locally finite. Thus, since $Z$ is contractible, there is an extension $\psi_{b a}\left[\mathrm{DD}\right.$, Lem. 4]. This contradicts the maximality of $\left(K, \psi_{. a}\right)$, so $K=J$. We define $\psi_{a a}:=\varphi_{a a a}$ and then $\psi_{a j}(u):=\varphi_{a j a}(u) \cdot g_{a j}(u) \psi_{a a}(u)$. $g_{a j}(u)^{-1} \psi_{j a}(u) \cdot g_{a j}(u)$, for $j \in J$. We let $J^{\prime}:=\{a\} \cup J$ and extend $\chi_{. .}$to $J^{\prime}$ by

$$
\chi_{i j}^{\prime}:=\left\{\begin{array}{ll}
\chi_{i j}, & \text { if } i, j \in J, \\
\psi_{i a}, & \text { if } j=a, i \in J, \\
\psi_{a a}, & \text { if } i=j=a, \\
\psi_{a j}, & \text { if } i=a, j \in J
\end{array} .\right.
$$

It is straight forward to check that $\left(J^{\prime}, \chi_{. .}^{\prime}\right) \in L$, and as clearly $\left(J, \chi_{. .}\right) \leq\left(J^{\prime}, \chi_{. .}^{\prime}\right)$ we have a contradiction. Hence $J=I$, and the lemma is proven.

Theorem 3.5 Let $G=\mathbb{R}^{n}, N=\mathbb{Z}^{n}$. Then every topological triple is almost strict, i.e. in this case $\operatorname{Top}^{\text {as }}(B)=\operatorname{Top}(B)$. Moreover, the group $Q_{\mathbb{R}}$ defined in diagram (33) vanishes, hence the three natural transformations of Theorem 3.4 reduce to one single transformation

$$
\delta: \text { Top } \rightarrow \text { Dyn }^{\dagger} .
$$

Proof : By the lemma above, it is sufficient to show that

$$
Z:=Z_{\text {cont }}^{1}\left(\mathbb{R}^{n}, \operatorname{Map}\left(\mathbb{T}^{n}, \mathrm{U}(1)\right)\right)
$$

is contractible. Then it follows that $\check{H}^{2}\left(B, \underline{Z}, g_{\text {.. }}\right)=\{1\}$ and every topological triple is almost strict. Because $Q_{\mathbb{R}}=\check{H}^{2}\left(B, \underline{Z}, g_{\text {.. }}\right)=\{1\}$, the torsor structures in Theorem 3.4 are trivial and we obtain a natural map $\delta(B): \operatorname{Top}(B) \rightarrow$ $\operatorname{Dyn}^{\dagger}(B)$.

We now show that $Z$ is contractible. For a cocycle $\alpha \in Z$ we have $\alpha(0)(z)=$ $\alpha(0+0)(z)=\alpha(0)(z) \alpha(0)(z)$ for all $z \in \mathbb{T}^{n}$, so $\alpha(0)(z)=1$ and each $\alpha(g)$ : $\mathbb{T}^{n} \rightarrow \mathrm{U}(1)$ is null homotopic as $\mathbb{R}^{n}$ is path connected. Thus

$$
Z=Z_{\text {cont }}^{1}\left(\mathbb{R}^{n}, \operatorname{Map}\left(\mathbb{T}^{n}, \mathrm{U}(1)\right)\right)=Z_{\text {cont }}^{1}\left(\mathbb{R}^{n}, \operatorname{Map}_{0}\left(\mathbb{T}^{n}, \mathrm{U}(1)\right)\right),
$$


for $\operatorname{Map}_{0}\left(\mathbb{T}^{n}, \mathrm{U}(1)\right):=\{f \simeq$ const. $\}$. But for each $\alpha: \mathbb{R}^{n} \rightarrow \operatorname{Map}_{0}\left(\mathbb{T}^{n}, \mathrm{U}(1)\right)$ there exists a lift $\bar{\alpha}$ in

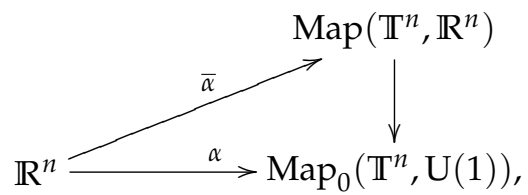

and because of $d \alpha=1$, we have $d \bar{\alpha}=m \in Z_{\text {cont }}^{2}\left(\mathbb{R}^{n}, \operatorname{Map}\left(\mathbb{T}^{n}, \mathbb{Z}\right)\right) \cong \mathbb{Z}$. Then $\alpha^{\prime}(g)(z):=\bar{\alpha}(g)(z)-m$ defines a unique element $\alpha^{\prime} \in Z_{\text {cont }}^{1}\left(\mathbb{R}^{n}, \operatorname{Map}_{0}\left(\mathbb{T}^{n}, \mathbb{R}\right)\right)$ such that $\alpha^{\prime}(g)(z) \mathbb{Z}=\alpha(g)(z)$. In fact, the mapping $\alpha \mapsto \alpha^{\prime}$ is a homoeomorphism

$$
Z_{\text {cont }}^{1}\left(\mathbb{R}^{n}, \operatorname{Map}_{0}\left(\mathbb{T}^{n}, \mathrm{U}(1)\right)\right) \rightarrow Z_{\text {cont }}^{1}\left(\mathbb{R}^{n}, \operatorname{Map}\left(\mathbb{T}^{n}, \mathbb{R}\right)\right),
$$

and the latter space is easily seen to be contractible by $h\left(t, \alpha^{\prime}\right)(g)(z):=t \alpha^{\prime}(g)(z)$, $t \in[0,1]$. This proves the theorem.

Corollary 3.2 Let $G=\mathbb{R}^{n}, N=Z^{n}$. Then a pair has an extension to a toplogical T-duality triple if and only if it has an extension to a dualisable dynamical triple.

Proof : This follows from Corollary 3.1 and Theorem 3.5.

So far we have seen that in the case of $G=\mathbb{R}^{n}, N=\mathbb{Z}^{n}$

$$
\operatorname{Top}^{\mathrm{im}}(B) \subset \operatorname{Top}^{\mathrm{s}}(B) \subset \operatorname{Top}^{\text {as }}(B) \stackrel{!}{=} \operatorname{Top}(B),
$$

and the composition of the natural transformations $\tau$ and $\delta$ is the identity transformation $\delta \circ \tau=\mathrm{id}: \mathrm{Dyn}^{\dagger} \rightarrow \mathrm{Dyn}^{\dagger}$. The opposite composition $J:=\tau \circ \delta$ : Top $\rightarrow$ Top is an idempotent $J \circ J=J$. Unfortunately, inside our local theory we won't be able to answer the question whether $J$ is the identity on Top, i.e. all inclusions in (41) are equalities and $\delta$ and $\tau$ are both equivalences of functors inverse to each other. Nevertheless we can give this appealing answer when we restrict the functors Top and $\mathrm{Dyn}^{\dagger}$ to the subcategory $\mathrm{CW} \subset$ \{bases spaces of CW-complexes (or, more general, of base spaces with the homotopy type of a CW-complex). The point is that we can use a main result of [BRS] about the representability of the functor Top $\left.\right|_{C W}=\operatorname{Triple}_{n}$. However, the statement therein is not completely precise. We restate it for convenience.

Lemma 3.9 ([BRS, Thm. 7.24]) The functor Top $\left.\right|_{C W}: \mathrm{CW} \rightarrow\{$ sets $\}$ is representable by a space $R_{n} \in \mathrm{CW}$, i.e. there is an equivalence of functors

$$
\Psi:\left.\left[., R_{n}\right] \cong \operatorname{Top}\right|_{\mathrm{CW}}(\text {. ) , }
$$

where the squared brackets denote the homotopy classes of continuous maps.

The definition of $\Psi$ is via pullback of a certain topological triple $x_{\text {univ }} \in \operatorname{Top}\left(R_{n}\right)$ (the universal triple) over $R_{n}$; for $B \in \mathrm{CW},[f] \in\left[B, R_{n}\right]$ it is

$$
\Psi(B)([f]):=f^{*} x_{\text {univ }} .
$$


Further, it is shown in [BRS, Sec. 4] that the space $R_{n}$ has an homotopy action of the so-called T-duality group $O(n, n, \mathbb{Z})$ which is the group of $2 n \times 2 n$ matrices that fix the form

$$
\mathbb{Z}^{2 n} \ni\left(a_{1}, \ldots, a_{n}, b_{1}, \ldots, b_{n}\right) \mapsto \sum a_{i} b_{i} \in \mathbb{Z} .
$$

So each element of $O(n, n, \mathbb{Z})$ defines a homotopy class of maps $R_{n} \rightarrow R_{n}$. In particular, the element

$$
\left(\begin{array}{ll}
\mathbf{0}_{n} & \mathbf{1}_{n} \\
\mathbf{1}_{n} & \mathbf{0}_{n}
\end{array}\right) \in O(n, n, \mathbb{Z})
$$

defines (the homotopy class of) a function $T: R_{n} \rightarrow R_{n}$. T is constructed such that $T \circ T=\operatorname{id}_{R_{n}}$ and such that the pullback $T^{*}: \operatorname{Top}\left(R_{n}\right) \rightarrow \operatorname{Top}\left(R_{n}\right)$ exchanges the underlying torus bundles ${ }^{13}$. To be precise, the construction of $T$ is such that if $[(\kappa,(P, E),(\widehat{P}, \widehat{E}))] \in \operatorname{Top}\left(R_{n}\right)$ is a topological triple, then $T^{*}$ maps this triple to a triple $\left[\left(\kappa^{\prime},\left(P^{\prime}, E^{\prime}\right),\left(\widehat{P}^{\prime}, \widehat{E}^{\prime}\right)\right)\right]$, such that $E^{\prime} \cong \widehat{E}$ and $\widehat{E}^{\prime} \cong E$. Note that

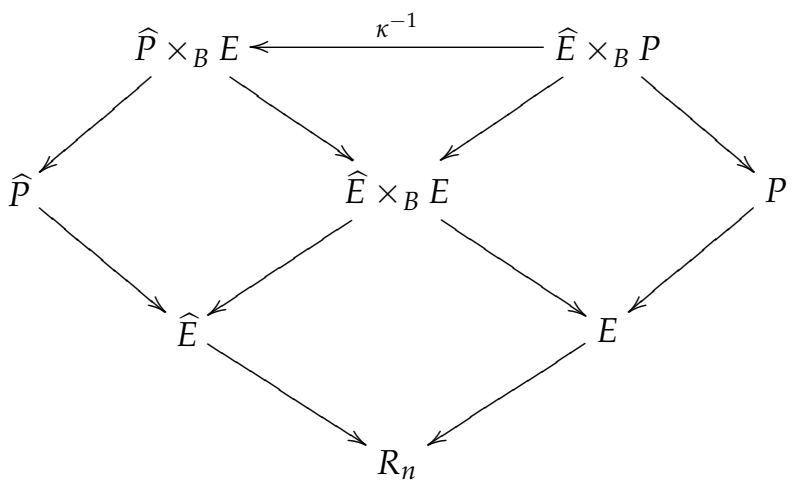

is not a topological triples as the inverse of $\kappa$ does not satisfy the Poincare condition, but also note that

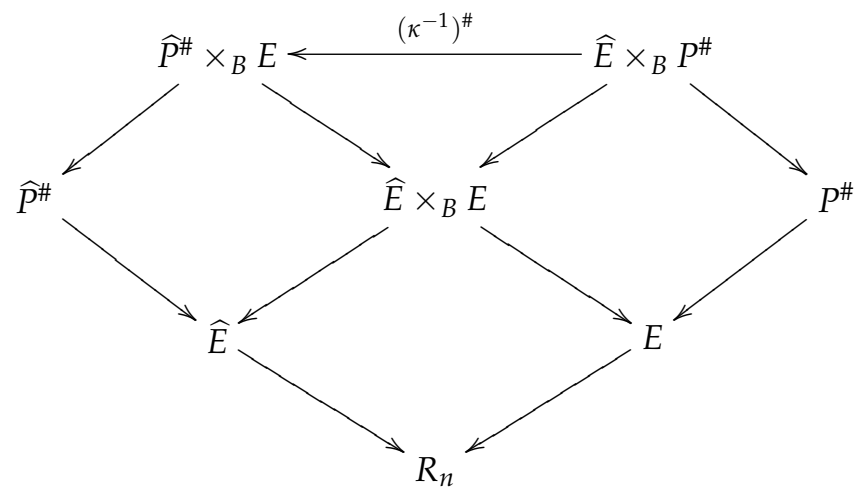

${ }^{13}$ This is meaningful, as the case of the groups $G=\mathbb{R}^{n}, N=\mathbb{Z}^{n}$ is self-dual, i.e. $G \cong \widehat{G}, N \cong N^{\perp}$. 
is a topological triple, wherein the superscript \# denotes the complex conjugate $^{14}$ bundles and isomorphism.

We claim that

$$
T^{*}[(\kappa,(P, E),(\widehat{P}, \widehat{E}))]=\left[\left(\left(\kappa^{-1}\right)^{\#},\left(\widehat{P}^{\#}, \widehat{E}\right),\left(P^{\#}, \widehat{E}\right)\right)\right] .
$$

In fact, by [BRS, Prop. 7.4] the set of topological triples over $R_{n}$ with fixed torus bundles $E^{\prime}, \widehat{E}^{\prime}$ is a torsor over $H^{3}\left(R_{n}, \mathbb{Z}\right)$ and by [BRS, Lem. 3.3] $H^{3}\left(R_{n}, \mathbb{Z}\right)=0$. In other words, there only exists one triple over $R_{n}$ which has underlying torus bundles $E^{\prime} \cong \widehat{E}$ and $\widehat{E}^{\prime} \cong E$, hence equation (42) is valid.

The properties of the map $T$ which is called universal T-duality enables us to prove the statement indicated above:

Theorem 3.6 Let $G=\mathbb{R}^{n}$ and $N=\mathbb{Z}^{n}$. Then we have an equivalence of functors

$$
\left.\delta\right|_{\mathrm{CW}}:\left.\left.\mathrm{Top}\right|_{\mathrm{CW}} \cong \mathrm{Dyn}^{\dagger}\right|_{\mathrm{CW}}:\left.\tau\right|_{\mathrm{CW}} .
$$

In particular

$$
\operatorname{Top}^{\mathrm{im}}(B)=\operatorname{Top}^{\mathrm{s}}(B)=\operatorname{Top}^{\mathrm{as}}(B)=\operatorname{Top}(B),
$$

for all $B \in \mathrm{CW}$.

Proof : It suffices to show that $J(B)=$ id, for all $B \in \mathrm{CW}$. $J$ is a natural transformation, so

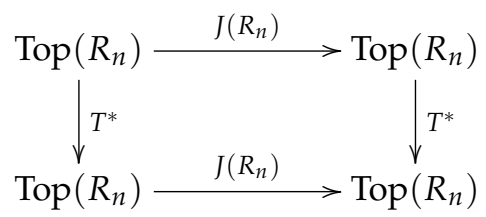

is a commutative diagram. But by construction $J(B)$ does not change the underlying pairs of the corresponding topological triples, for any $B$, so by the commutativity of the diagram $J\left(R_{n}\right)$ at least does not change the underlying pairs and underlying dual pairs of the corresponding triples. That $J\left(R_{n}\right)$ is the identity, i.e. it does not change the equivalence class of the isomorphisms $\kappa$, follows from the trivial $H^{3}\left(R_{n}, \mathbb{Z}\right)$-torsor structure of the set of topological triples with fixed torus bundles. Hence $J\left(R_{n}\right)=$ id.

Now, let $B \in \mathrm{CW}$ and $x \in \operatorname{Top}(B)$ be any topological triple. By the universal property of $x_{\text {univ }}$, there is $f: B \rightarrow R_{n}$ such that $x=f^{*} x_{\text {univ }}$ and, by naturality of $J$,

$$
J(B)(x)=J(B)\left(f^{*} x_{\text {univ }}\right)=f^{*}\left(J\left(R_{n}\right)\left(x_{\text {univ }}\right)\right)=f^{*}\left(x_{\text {univ }}\right)=x .
$$

So $J(B)=$ id, for all $B \in \mathrm{CW}$.

\footnotetext{
${ }^{14}$ Complex conjugation may be defined by taking $\mathcal{H}=l^{2} \mathbb{N}$ and then defining the complex conjugate bundles and isomorphism by complex conjugation of the local transition functions and local isomorphisms.
} 
We end this section with a remark on homotopic deckers. Its content is based on the fact that the functor Top $\left.\right|_{\mathrm{CW}} \cong\left[., R_{n}\right]$ is homotopy invariant.

Remark 3.4 Let $(P, E)$ be a pair over $B \in C W$, then $(P \times[0,1], E \times[0,1])$ is a pair over $B \times[0,1]$. Let $(\rho, P \times[0,1], E \times[0,1])$ be a dualisable dynamical triplein other words $\rho$ is a homotopy of dualisable deckers $\rho_{0}$ and $\rho_{1}$ on $(P, E)$ - then the classes $\left[\left(\rho_{0}, P, E\right)\right]$ and $\left[\left(\rho_{1}, P, E\right)\right]$ coincide.

Proof: As the the inclusions $i_{t}: B \hookrightarrow B \times[0,1], t=0,1$, are homotopic, we have an equality

$$
\begin{aligned}
\tau(B)\left(\left[\left(\rho_{0}, P, E\right)\right]\right) & =i_{0}^{*} \tau(B \times[0,1])([(\rho, P \times[0,1], E \times[0,1])]) \\
& =i_{1}^{*} \tau(B \times[0,1])([(\rho, P \times[0,1], E \times[0,1])]) \\
& =\tau(B)\left(\left[\left(\rho_{1}, P, E\right)\right]\right),
\end{aligned}
$$

and the claim follows from $\delta(B) \circ \tau(B)=$ id.

\subsection{The Structure of the Associated $C^{*}$-Dynamical Systems}

Let $(\rho, P, E)$ be a dualisable dynamical triple and let us denote by $F:=P \times_{\mathrm{PU}}(\mathcal{H})$ $\mathcal{K}(\mathcal{H})$ the associated $C^{*}$-bundle. The decker $\rho$ induces a $G$-action on $F$ by $[x, K] \cdot g:=[\rho(x, g), K] x \in P, K \in \mathcal{K}(\mathcal{H})$. This action defines another action $\alpha^{\rho}$ of $G$ on the $C^{*}$-algebra of sections $\Gamma(E, F)$ such that $\left(\Gamma(E, F), G, \alpha^{\rho}\right)$ becomes a $C^{*}$-dynamical system. This action is given by

$$
\left(\alpha^{\rho} g s\right)(e):=s(e \cdot g N) \cdot(-g), \quad e \in E, g \in G, s \in \Gamma(E, F) .
$$

In the same manner we obtain a dual $C^{*}$-dynamical system $\left(\Gamma(\widehat{E}, \widehat{F}), \widehat{G}, \alpha^{\hat{\rho}}\right)$ for the associated $C^{*}$-bundle $\widehat{F}:=\widehat{P} \times \mathrm{PU}\left(L^{2}(G / N) \otimes \mathcal{H}\right) \mathcal{K}\left(L^{2}(G / N) \otimes \mathcal{H}\right)$ of the dual triple $(\hat{\rho}, \widehat{E}, \widehat{P})$ of $(\rho, E, P)$. The essence of this section is that we can establish an isomorphism of $C^{*}$-dynamical systems from the crossed product ${ }^{15}$ of the first to the dual $C^{*}$-dynamical system (Thm. 3.8)

$$
\left(G \times \alpha^{\rho} \Gamma(E, F), \widehat{G}, \widehat{\alpha^{\rho}}\right) \cong\left(\Gamma(\widehat{E}, \widehat{F}), \widehat{G}, \alpha^{\hat{\rho}}\right) .
$$

We are going to calculate the crossed product under a series of isomorphisms which again will be a local calculation. We start with the description of the simplest case, namely $B$ being a point.

In the situation of the trivial pair over the point $B=\{*\}$ the sections can be identified with the continuous functions

$$
\Gamma(\text { triv. pair }) \cong C(G / N, \mathcal{K}(\mathcal{H})),
$$

\footnotetext{
${ }^{15}$ See the section on crossed products on page 92 for notation.
} 
since any section $s: G / N \rightarrow G / N \times \mathcal{K}(\mathcal{H})$ is uniquely given by a function $f$ such that $s(z)=(z, f(z))$. Then the action of $g \in G$ on such a function $f$ is obtained from

$$
\begin{aligned}
\left(\alpha_{g}^{\rho} s\right)(z) f & =s(z+g N) \cdot(-g) \\
& =(z+g N, f(z+g N) \cdot(-g) \\
& =(z, \mu(-g, z+g N)(f(z+g N))) \\
& =\left(z,(\mu(g, z))^{-1}(f(z+g N))\right) \\
& =:\left(z,\left(\alpha_{g}^{\mu} f\right)(z)\right)
\end{aligned}
$$

for the 1-cocycle $\mu$ determined by $\rho$.

For the $C^{*}$-algebra of the dual trivial pair over the point we have

$$
\Gamma(\text { dual triv. pair }) \cong C\left(\widehat{G} / N^{\perp}, \mathcal{K}\left(L^{2}\left(N^{\perp}, \mathcal{H}\right)\right)\right) .
$$

It will be convenient at some point to deal with the Hilbert space $L^{2}(\widehat{G / N}, \mathcal{H})$ rather than with $L^{2}(G / N) \otimes \mathcal{H}$. So we have to transform the cocycle $\hat{\mu}$ of the dual decker $\hat{\rho}$ from equation (20) by Fourier transform $\mathcal{F}: L^{2}(G / N) \rightarrow$ $L^{2}(\widehat{G / N})$,

$$
\left(\mathcal{F}_{*} \hat{\mu}\right)(\chi, \hat{z}):=\left(\mathcal{F} \otimes \mathbb{1}_{\mathcal{H}}\right) \circ \hat{\mu}(\chi, \hat{z}) \circ\left(\mathcal{F}^{-1} \otimes \mathbb{1}_{\mathcal{H}}\right),
$$

and as in eq. (43) this gives us an action

$$
\alpha^{\mathcal{F}_{*} \hat{\mu}}: \widehat{G} \rightarrow \operatorname{Aut}\left(C\left(\widehat{G} / N^{\perp}, \mathcal{K}\left(L^{2}\left(N^{\perp}, \mathcal{H}\right)\right)\right)\right)
$$

by $\left(\alpha_{*}^{\mathcal{F}_{*} \hat{\mu}} \chi f\right)(\hat{z}):=\left(\mathcal{F}_{*} \hat{\mu}\right)(\chi)(\hat{z})^{-1}\left(f\left(\hat{z}+\chi N^{\perp}\right)\right)$, for $\chi \in \widehat{G}, \hat{z} \in \widehat{G} / N^{\perp}$.

The next lemma is a simple link between $\mathcal{F}_{*} \hat{\mu}$ and the left regular representation $\lambda_{\widehat{G / N}}$ on $L^{2}(\widehat{G / N})$. $\lambda_{\widehat{G / N}} \in \operatorname{Hom}\left(\widehat{G / N}, \mathrm{U}\left(L^{2}(\widehat{G / N})\right)\right)$ is a continuous homomorphism.

Lemma 3.10 There exists a continuous extension $\Lambda \in \operatorname{Hom}\left(\widehat{G}, \mathrm{U}\left(L^{2}(\widehat{G / N})\right)\right)$ such that

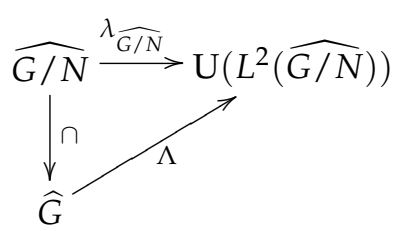

commutes.

Proof : Let $\beta \in \widehat{G / N}$. The Fourier transform $\mathcal{F}$ turns $\lambda_{\widehat{G / N}}(\beta)$ into the multiplication operator $\left\langle-\beta,,_{-}\right\rangle=\mathcal{F}^{-1} \circ \lambda^{\perp}(\beta) \circ \mathcal{F} \in \mathrm{U}\left(L^{2}(G / N)\right)$. Let $\sigma: G / N \rightarrow G$ be our chosen Borel section. We define an extension of $\beta \mapsto\left\langle-\beta\right.$, s $\left._{-}\right\rangle$by $\widehat{G} \ni$ $\chi \mapsto\langle\chi,-\sigma(-)\rangle$. This is a strongly continuous homomorphism, and it follows that $\Lambda(\chi):=\mathcal{F} \circ\langle\chi,-\sigma(-)\rangle \circ \mathcal{F}^{-1}$ gives us the desired homomorphism $\Lambda: \widehat{G} \rightarrow \mathrm{U}\left(L^{2}(\widehat{G / N})\right)$. 
With the notation of this lemma and equation (20) we can reformulate the defintion of $\mathcal{F}_{*} \hat{\mu}$, we have $\left(\mathcal{F}_{*} \hat{\mu}\right)(\chi, \hat{z})=\operatorname{Ad}\left(\Lambda(\chi) \otimes \mathbb{1}_{\mathcal{H}}\right)$ which is the adequate formula for the next theorem. Therein $\alpha \mapsto \alpha^{\perp}$ denotes the natural isomorphism $\widehat{G / N} \cong N^{\perp} \subset \widehat{G}$ which is defined by $\left\langle\alpha^{\perp}, g\right\rangle:=\langle\alpha, g N\rangle$.

Theorem 3.7 Assume the considered dynamical triple over $B=\{*\}$ is dualisable. Then there is an isomorphism of $C^{*}$-dynamical systems

$$
\left(G \times \alpha_{\alpha^{\mu}} C(G / N, \mathcal{K}(\mathcal{H})), \widehat{G}, \widehat{\alpha^{\mu}}\right) \stackrel{\cong}{\longrightarrow}\left(C\left(\widehat{G} / N^{\perp}, \mathcal{K}\left(L^{2}(\widehat{G / N}, \mathcal{H})\right)\right), \widehat{G}, \alpha^{\mathcal{F}_{*} \hat{\mu}}\right) .
$$

Proof : First note that naturally

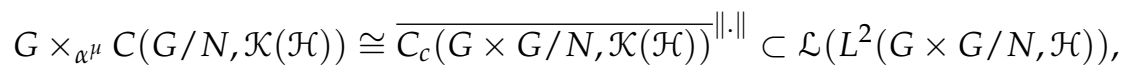

wherein $f \in C_{c}(G \times G / N, \mathcal{K}(\mathcal{H}))$ acts on $F \in L^{2}(G \times G / N, \mathcal{H})$ by

$$
(f \times F)(g, z)=\int_{G}(\mu(-g)(z))^{-1}(f(h, z-g N)) F(g-h, z) d h .
$$

We assume the dynamical triple to be dualisable, so we can lift $\mu$ to a unitary (Borel) cocycle $\bar{\mu}: G \times G / N \rightarrow \mathrm{U}(\mathcal{H})$. Note that for $K \in \mathcal{K}(\mathcal{H})$ )

$$
\bar{\mu}(-g, z) \mu(-g, z)^{-1}(K)=K \bar{\mu}(-g, z): \mathcal{H} \rightarrow \mathcal{H} .
$$

We now define a unitary isomorphism

$$
u: L^{2}(G \times G / N, \mathcal{H}) \rightarrow L^{2}\left(\widehat{G}, L^{2}(\widehat{G / N}, \mathcal{H})\right)
$$

by the composition $u:=$ shift $\circ($ Fourier trans. $) \circ$ mult $_{\bar{\mu}(-,-)}$, explicitely

$$
\begin{aligned}
(u F)(\chi)(\alpha) & =(\widehat{\mu(-,-)} F)\left(\chi+\alpha^{\perp},-\alpha\right) \\
& =\int_{G \times G / N}\left\langle\chi+\alpha^{\perp}, g\right\rangle\langle-\alpha, z\rangle \bar{\mu}(-g, z) F(g, z) d(g, z),
\end{aligned}
$$

for $\chi \in \widehat{G}, \alpha \in \widehat{G / N}$. The next step is to calculate $u\left(f \times_{-}\right) u^{-1}$. This is straightforward, but to keep the calculation readable we first introduce some short hands: $\bar{\mu} F(g, z):=\bar{\mu}(-g, z) F(g, z), f_{\bar{\mu}}(g, z):=f(g, z) \bar{\mu}(g, z)^{-1}$ and $\hat{f}^{2}$ the 
Fourier transform in the second, the $G / N$ variable only. Now

$$
\begin{aligned}
& u(f \times F)(\chi)(\alpha) \\
& =\int_{G \times G / N} \int_{G}\left\langle\chi+\alpha^{\perp}, g\right\rangle\langle-\alpha, z\rangle f(h, z-g N) \bar{\mu}(-g, z) F(g-h, z) d h d(g, z) \\
& =\int_{G \times G / N} \int_{G}\left\langle\chi+\alpha^{\perp}, g\right\rangle\langle-\alpha, z\rangle f_{\bar{\mu}}(h, z-g N) \bar{\mu} F(g-h, z) d h d(g, z) \\
& =\int_{G \times G} \int_{\widehat{G / N}}\left\langle\chi+\alpha^{\perp}, g\right\rangle{\widehat{f_{\bar{\mu}}}}^{2}(h, \beta)\langle\beta, g N\rangle \widehat{\bar{\mu}}^{2}(g-h,(-\alpha)-\beta) d \beta d(h, g) \\
& =\int_{\widehat{G / N}} \int_{G} \widehat{f}_{\bar{\mu}}^{2}(h, \beta)\left\langle\chi+\alpha^{\perp}+\beta^{\perp}, h\right\rangle \widehat{\bar{\mu} F}\left(\chi+\alpha^{\perp}+\beta^{\perp},(-\alpha)-\beta\right) d h d \beta \\
& =\int_{\widehat{G / N}} \widehat{f_{\bar{\mu}}}\left(\chi+\alpha^{\perp}+\beta^{\perp}, \beta\right) \widehat{\bar{\mu} F}\left(\chi+\alpha^{\perp}+\beta^{\perp},(-\alpha)-\beta\right) d \beta \\
& =\int_{\widehat{G / N}} \underbrace{\widehat{f_{\bar{\mu}}}\left(\chi+\gamma^{\perp}, \gamma-\alpha\right)} \underbrace{\widehat{\bar{\mu} F}\left(\chi+\gamma^{\perp},-\gamma\right)} d \gamma, \\
& =: f^{\bar{\mu}}(\chi)(\alpha, \gamma) \quad=u F(\chi)(\gamma)
\end{aligned}
$$

and we think of $f^{\bar{\mu}}$ as a continuous family of Hilbert-Schmidt operators $f^{\bar{\mu}}$ : $\widehat{G} \rightarrow \mathcal{K}\left(L^{2}(\widehat{G / N}, \mathcal{H})\right)$, i.e. we do not decide in notation between the operator $f^{\bar{\mu}}(\chi)$ and its integral kernel. From the definition of the kernel $f^{\bar{\mu}}$ we obtain $f^{\bar{\mu}}\left(\chi+\beta^{\perp}\right)(\alpha, \gamma)=f^{\bar{\mu}}(\chi)(\alpha+\beta, \gamma+\beta), \alpha, \gamma, \beta \in \widehat{G / N}$, so the operator $f^{\bar{\mu}}(\chi)$ satisfies the identity

$$
f^{\bar{\mu}}\left(\chi+\beta^{\perp}\right)=\left(\lambda_{\widehat{G / N}}(\beta)^{-1} \otimes \mathbb{1}\right) f^{\bar{\mu}}(\chi)\left(\lambda_{\widehat{G / N}}(\beta) \otimes \mathbb{1}\right) \in \mathcal{K}\left(L^{2}(\widehat{G / N}, \mathcal{H})\right),
$$

for the left regular representation $\lambda_{\widehat{G / N}}$. We now use the chosen extension $\Lambda \in$ $\operatorname{Hom}\left(\widehat{G}, \operatorname{PU}\left(L^{2}(\widehat{G / N}, \mathcal{H})\right)\right)$ from Lemma 3.10 to define $T_{\bar{\mu}}: C_{c}(G \times G / N, \mathcal{K}(\mathcal{H})) \rightarrow$ $C\left(\widehat{G} / N^{\perp}, \mathcal{K}\left(L^{2}(\widehat{G / N}, \mathcal{H})\right)\right)$ by

$$
\begin{aligned}
\left(T_{\bar{\mu}} f\right)\left(\chi N^{\perp}\right) & :=(\Lambda(\chi) \otimes \mathbb{1}) f^{\bar{\mu}}(\chi)\left(\Lambda(\chi)^{-1} \otimes \mathbb{1}\right) \\
& =\operatorname{Ad}(\Lambda(\chi) \otimes \mathbb{1})) f^{\bar{\mu}}(\chi) .
\end{aligned}
$$

It is now a lengthy but straight forward calculation to check that, firstly, $T_{\bar{\mu}}$ commutes with the ${ }^{*}$-operation, i.e. $T_{\bar{\mu}}\left(f^{\times}\right)(\hat{z})=\left(T_{\bar{\mu}} f\right)(\hat{z})^{*}$, for $\hat{z} \in \widehat{G} / N^{\perp}$ and $f^{\times}(g, z)=\mu(g)(z)^{-1}\left(f(-g, z+g N)^{*}\right)$. Secondly, $T_{\bar{\mu}}$ preserves the product, i.e. $T_{\bar{\mu}}\left(f_{1} \times f_{2}\right)(\hat{z})=T_{\bar{\mu}} f_{1}(\hat{z}) T_{\bar{\mu}} f_{2}(\hat{z})$. Thirdly, $T_{\bar{\mu}}$ preserves the norm, i.e. $\left\|f \times{ }_{-}\right\|=$ $\sup _{\hat{z} \in \widehat{G} / N^{\perp}}\left\|T_{\bar{\mu}} f(\hat{z})\right\|$.

Moreover $T_{\bar{\mu}}$ has dense image, so it extends uniquely to a $C^{*}$-algebra isomorphism

$$
T_{\bar{\mu}}: G \times{ }_{\alpha^{\mu}} C(G / N, \mathcal{K}(\mathcal{H})) \rightarrow C\left(\widehat{G} / N^{\perp}, \mathcal{K}\left(L^{2}(\widehat{G / N}, \mathcal{H})\right)\right) .
$$


It remains to show that $T_{\bar{\mu}}\left(\widehat{\alpha}_{\chi} f\right)=\alpha^{\mathcal{F}_{*} \hat{\mu}} \chi\left(T_{\bar{\mu}} f\right)$ :

By definition ${\widehat{\alpha^{\mu}}}_{\chi}$ is just the multiplication with the character $\chi$, so the Fourier transform gives us simply a shift in the argument by $\chi$. We get

$$
\begin{aligned}
& T_{\bar{\mu}}\left(\widehat{\alpha}^{\mu} \chi f\right)\left(\chi^{\prime} N^{\perp}\right)=\operatorname{Ad}\left(\Lambda\left(\chi^{\prime}\right) \otimes \mathbb{1}\right) f^{\bar{\mu}}\left(\chi^{\prime}+\chi\right) \\
& =\operatorname{Ad}\left(\Lambda(\chi)^{-1} \otimes \mathbb{1}\right) \operatorname{Ad}\left(\Lambda\left(\chi^{\prime}+\chi\right) \otimes \mathbb{1}\right)\left(f^{\bar{\mu}}\left(\chi^{\prime}+\chi\right)\right) \\
& =\left(\mathcal{F}_{*} \hat{\mu}\right)(\chi)\left(\chi^{\prime} N^{\perp}\right)^{-1} T_{\bar{\mu}} f\left(\chi^{\prime} N^{\perp}+\chi N^{\perp}\right) \\
& =\alpha^{\mathcal{F}_{*} \hat{\mu}} \chi\left(T_{\bar{\mu}} f\right)\left(\chi^{\prime} N^{\perp}\right) \text {. }
\end{aligned}
$$

The discussion in the previous theorem will serve as a description of the local situation of the general case. Let $(\rho, P, E)$ be a dualisable dynamical triple, and let $(\hat{\rho}, \widehat{P}, \widehat{E})$ be its dual. We defined the associated $C^{*}$-bundles $F, \widehat{F}$ above. Recall the definition of $\hat{g}_{j i}$ out of $\phi_{j i}$ (p. 39). A priori there need not exist a continuous lift $\varphi_{j i}$ in the diagram

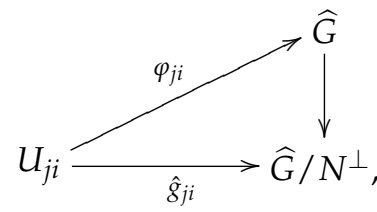

but by Lemma A. 8 we assume without restriction that our atlas is sufficiently refined such that $\varphi_{j i}$ exists. We define $\varphi_{j i}^{\prime}(u)(g N):=\phi_{j i}(u)(g, 0)\left\langle\varphi_{j i}(u), g\right\rangle^{-1}$. Although the function $u \mapsto \varphi_{j i}^{\prime}(u) \in L^{\infty}(G / N, \mathrm{U}(1))$ need not to be continuous, the function $u \mapsto \operatorname{Ad}\left(\varphi_{j i}^{\prime}(u)\right) \in \mathrm{PL}^{\infty}(G / N, \mathrm{U}(1))$ is continuous by Lemma 3.1, and we have the identity

$$
\begin{aligned}
\phi_{n m}(u)(g, h N) & =\phi_{n m}(u)(g+h, 0) \phi_{n m}(u)(h, 0)^{-1} \\
& =\varphi_{n m}(u)(g+h) \varphi_{n m}^{\prime}(u)(g N+h N) \varphi_{n m}(u)(h)^{-1} \varphi_{n m}^{\prime}(u)(h N)^{-1} \\
& =\varphi_{n m}(u)(g) d\left(\varphi_{n m}^{\prime}(u)\right)(g, h N) .
\end{aligned}
$$

Theorem 3.8 There is an isomorphism of $C^{*}$-dynamical systems

$$
\left(G \times \alpha_{\alpha^{\rho}} \Gamma(E, F), \widehat{G}, \widehat{\alpha^{\rho}}\right) \stackrel{\cong}{\longrightarrow}\left(\Gamma(\widehat{E}, \widehat{F}), \widehat{G}, \alpha^{\hat{\rho}}\right) .
$$

Proof : We generalise the proof of Theorem 3.7. The sections $s \in \Gamma(E, F)$ are in one to one correspondence with families of functions $s_{i} \in C\left(U_{i} \times G / N, \mathcal{K}(\mathcal{H})\right)$, $i \in I$, which satisfy

$$
s_{i}(u, z)=\zeta_{j i}(u)(z)^{-1}\left(s_{j}\left(u, g_{j i}(u)+z\right)\right), \quad u \in U_{i j}, z \in G / N .
$$


It is $G \times{ }_{\alpha \phi} \Gamma(E, F)=\overline{C_{c}(G, \Gamma(E, F))}\|\cdot\|$, and for each chart $U_{i}$ of the pair $(P, E)$ we have induced restriction maps

$$
\begin{aligned}
G \times{ }_{\alpha^{\rho}} \Gamma(E, F) & \rightarrow G \times{ }_{\alpha^{\mu_{i}}} C\left(U_{i} \times G / N, \mathcal{K}(\mathcal{H})\right) \\
\cup & \cup \\
C_{c}(G, \Gamma(E, F)) & \rightarrow C\left(U_{i}, C_{c}(G \times G / N, \mathcal{K}(\mathcal{H}))\right) . \\
f & \mapsto f_{i}
\end{aligned}
$$

The triple $(\rho, P, E)$ is dualisable, so we can lift (if necessary after a refinement of the atlas) the cocycles $\mu_{i}$ to unitary Borel cocycles $\bar{\mu}_{i}: U_{i} \rightarrow Z_{\text {Bor }}^{1}\left(G, L^{\infty}(G / N, \mathrm{U}(\mathcal{H}))\right)$. For each such $\bar{\mu}_{i}$ we define an operator

$$
T_{i}: C\left(U_{i}, C_{c}(G \times G / N, \mathcal{K}(\mathcal{H}))\right) \rightarrow C\left(U_{i} \times \widehat{G} / N^{\perp}, \mathcal{K}\left(L^{2}(\widehat{G / N}, \mathcal{H})\right)\right)
$$

as in equation (45) by

$$
T_{i} f_{i}\left(u, \chi N^{\perp}\right):=\left(T_{\bar{\mu}_{i}}(u) f_{i}(u)\right)\left(\chi N^{\perp}\right)=\operatorname{Ad}(\Lambda(\chi)) f_{i}(u)^{\bar{\mu}_{i}(u)}(\chi),
$$

wherein we used the notation of equation (44). In view of equation (47) we are interested in the relation between $T_{i} f_{i}$ and $T_{j} f_{j}$ on overlaps $U_{i j}$. So let $u \in U_{j i}$, then

$$
\begin{aligned}
& f_{i}(u)^{\bar{\mu}_{i}(u)}(\chi)(\alpha, \gamma) \\
& \stackrel{(44)}{=} \quad \int_{G \times G / N} f_{i}(u)(g, z) \bar{\mu}_{i}(u)(g, z)^{-1}\left\langle\chi+\gamma^{\perp}, g\right\rangle\langle\gamma-\alpha, z\rangle d(g, z) \\
& \stackrel{(47) \text { and def. of } \phi_{j i}}{=} \int_{G \times G / N} \bar{\zeta}_{j i}(u)(z)^{*} f_{j}(u)\left(g, g_{j i}(u)+z\right) \bar{\mu}_{j}(u)\left(g, g_{j i}(u)+z\right)^{-1} \\
& \bar{\zeta}_{j i}(u)(g N+z) \phi_{j i}(u)(g, z)\left\langle\chi+\gamma^{\perp}, g\right\rangle\langle\gamma-\alpha, z\rangle d(g, z) \\
& =\quad \int_{G \times G / N} \bar{\zeta}_{j i}^{\varphi}(u)(z)^{*} f_{j}(u)_{\bar{\mu}_{j}(u)}\left(g, g_{j i}(u)+z\right) \\
& \bar{\zeta}_{j i}^{\varphi}(u)(g N+z)\left\langle\varphi_{j i}(u)+\chi+\gamma^{\perp}, g\right\rangle\langle\gamma-\alpha, z\rangle d(g, z) \\
& =\text { : }
\end{aligned}
$$

The notation $f_{j}(u)_{\bar{\mu}_{j}(u)}$ is as in the previous theorem, and $\bar{\zeta}_{j i}^{\varphi}(u)(z):=\bar{\zeta}_{j i}(u)(z) \varphi_{j i}^{\prime}(u)(z)$.

Since $G / N$ is compact and $\bar{\zeta}_{j i}(u)(z) \in \mathrm{U}(\mathcal{H})$ it follows that $\bar{\zeta}_{j i}^{\varphi}(u)(-) v \in$ $L^{2}(G / N, \mathcal{H})$, for each $v \in \mathcal{H}$. So it possesses a Fourier decomposition

$$
\bar{\zeta}_{j i}^{\varphi}(u)(g N+z)=\int_{\widehat{G / N}} \widehat{\overline{\zeta_{j i}^{\varphi}}(u)}(\delta)\langle-\delta, z\rangle\left\langle-\delta^{\perp}, g\right\rangle d \delta
$$


and

$$
\bar{\zeta}_{j i}^{\varphi}(u)(z)^{*}=\int_{\widehat{G / N}} \widehat{\bar{\zeta}_{j i}^{\varphi}(u)}(\varepsilon)^{*}\langle\varepsilon, z\rangle d \varepsilon,
$$

for some $\widehat{\bar{\zeta}_{j i}^{\varphi}(u)}\left({ }_{-}\right): \widehat{G / N} \rightarrow \mathcal{L}(\mathcal{H})$. The calculation continues inserting this into the previous equation,

$$
\begin{aligned}
& \mathbf{q}= \int_{\widehat{G / N}} \int_{\widehat{G / N}} \widehat{\overline{\bar{\zeta}_{j i}^{\varphi}(u)}}(\varepsilon)^{*} f_{j} \widehat{(u)_{\bar{\mu}_{j}}(u)}\left(\varphi_{j i}(u)+\chi+\gamma^{\perp}-\delta^{\perp}, \gamma-\delta-\alpha+\varepsilon\right) \\
&\left\langle\gamma-\delta-\alpha+\varepsilon,-g_{j i}(u)\right\rangle \widehat{\bar{\zeta}_{i j}^{\varphi}(u)}(\delta) d \varepsilon d \delta \\
&=\int_{\widehat{G / N}} \int_{\widehat{G / N}} \widehat{\bar{\zeta}_{j i}^{\varphi}(u)}(\varepsilon)^{*} f_{j}(u)^{\bar{\mu}_{j}(u)}\left(\varphi_{j i}(u)+\chi\right)(\alpha-\varepsilon, \gamma-\delta) \\
&=\int_{\widehat{G / N}} \int_{\widehat{G / N}} \widehat{\bar{\zeta}_{j i}^{\varphi}(u)}\left(\alpha-\delta-\alpha+\varepsilon,-g_{j i}(u)\right\rangle \widehat{\bar{\zeta}}_{i j}^{\varphi}(u)(\delta) d \varepsilon d \delta \\
&\left\langle\delta^{\prime},-g_{j i}(u)\right\rangle \widehat{\bar{\zeta}_{i j}^{\varphi}(u)}\left(\gamma-\delta_{j i}^{\prime}\right) d \varepsilon^{\prime} d \delta^{\prime} .
\end{aligned}
$$

Now, bearing in mind that $f_{i}(u)^{\bar{\mu}_{i}(u)}(\chi)(\alpha, \gamma)$ is the kernel of the integral operator $f_{i}(u)^{\bar{\mu}_{i}(u)}(\chi) \in \mathcal{K}\left(L^{2}(\widehat{G / N}, \mathcal{H})\right)$, the last expression becomes

$$
\begin{aligned}
f_{i}(u)^{\bar{\mu}_{i}(u)}(\chi) & =\eta_{j i}(u)^{*} f_{j}(u)^{\bar{\mu}_{i}(u)}\left(\varphi_{j i}(u)+\chi\right) \eta_{j i}(u) \\
& =\operatorname{Ad}\left(\eta_{j i}(u)^{*}\right)\left(f_{j}(u)^{\bar{\mu}_{j}(u)}\left(\varphi_{j i}(u)+\chi\right)\right) \in \mathcal{K}\left(L^{2}(\widehat{G / N}, \mathcal{H})\right),
\end{aligned}
$$

wherein $\eta_{j i}(u) \in \mathrm{U}\left(L^{2}\left(N^{\perp}, \mathcal{H}\right)\right)$ is the composition of the two operators

$$
\begin{aligned}
L^{2}(\widehat{G / N}, \mathcal{H}) & \rightarrow L^{2}(\widehat{G / N}, \mathcal{H}) \\
F & \mapsto \overline{\bar{\zeta}}_{j i}^{\varphi}(u)\left(-_{-}\right) * F: \beta \mapsto \int_{\widehat{G / N}} \widehat{\overline{\bar{\zeta}_{j i}^{\varphi}}}(u)(\beta-\alpha) F\left(\alpha^{\perp}\right) d \alpha \\
\text { and } & \\
L^{2}(\widehat{G / N}, \mathcal{H}) & \rightarrow L^{2}(\widehat{G / N}, \mathcal{H}) \\
F & \mapsto\left(\beta \mapsto\left\langle\beta,-g_{j i}(u)\right\rangle F(\beta)\right) .
\end{aligned}
$$

Note that both of these operators are in fact unitary, so $\eta_{j i}(u)$ is. The calculation 
done so far can now give us the relation we are looking for

$$
\begin{aligned}
T_{i} f_{i}\left(u, \chi N^{\perp}\right)= & \operatorname{Ad}(\Lambda(\chi) \otimes \mathbb{1}) f_{i}(u)^{\bar{\mu}_{i}(u)}(\chi) \\
= & \operatorname{Ad}(\Lambda(\chi) \otimes \mathbb{1}) \operatorname{Ad}\left(\eta_{j i}(u)^{-1}\right) f_{j}(u)^{\bar{\mu}_{j}(u)}\left(\varphi_{j i}(u)+\chi\right) \\
= & \underbrace{\operatorname{Ad}\left((\Lambda(\chi) \otimes \mathbb{1}) \eta_{j i}(u)^{-1}\left(\Lambda\left(\varphi_{j i}(u)+\chi \hat{F}_{j i}\right)(u)\left(\chi N^{\perp}\right)^{-1} \otimes \mathbb{1}\right)\right)}_{=:} \\
& \operatorname{Ad}\left(\Lambda\left(\varphi_{j i}(u)+\chi\right) \otimes \mathbb{1}\right) f_{j}(u)^{\bar{\mu}_{j}(u)}\left(\varphi_{j i}(u)+\chi\right) \\
= & \left(\mathcal{F}_{*} \hat{\zeta}_{j i}\right)(u)\left(\chi N^{\perp}\right)^{-1} T_{j} f_{j}(u \underbrace{\varphi_{j i}(u) N^{\perp}}_{=\hat{g}_{j i}}+\chi N^{\perp}) .
\end{aligned}
$$

$\left(\mathcal{F}_{*} \hat{\zeta}_{j i}\right)(u)\left(\chi N^{\perp}\right)$ is in fact well defined for $\chi N^{\perp} \in \widehat{G} / N^{\perp}$, since $\Lambda\left(\chi+\gamma^{\perp}\right)=$ $\Lambda(\chi) \Lambda\left(\gamma^{\perp}\right)=\Lambda(\chi) \lambda_{\widehat{G / N}}(\gamma)$, and the left regular representation operator $\lambda_{\overparen{G / N}}(\gamma)$ commutes with the convolution operator (48) and commutes with the multiplication operator (49) up to $\left\langle\gamma,-g_{j i}(u)\right\rangle$, a $\mathrm{U}(1)$-valued multiple of the identity. Hence the commutator of $\operatorname{Ad}\left(\Lambda\left(\gamma^{\perp}\right) \otimes \mathbb{1}\right)$ and $\operatorname{Ad}\left(\eta_{j i}(u)\right)$ vanishes in $\operatorname{PU}\left(L^{2}(\widehat{G / N}, \mathcal{H})\right)$.

In view of equation (47), equation (50) shows that the family $\left\{T_{i} f_{i}\right\}_{i \in I}$ defines a section in a $\mathcal{K}\left(L^{2}(\widehat{G / N}, \mathcal{H})\right)$-bundle over $E$ with transition functions $\mathcal{F}_{*} \hat{\zeta}_{j i}$. Up to Fourier transform, this bundle is nothing but $\widehat{F}$ itself, for we find

$$
\begin{aligned}
& \left(\mathcal{F}_{*} \hat{\zeta}_{j i}\right)(u)(\hat{z}) \\
= & \operatorname{Ad}\left((\mathcal{F} \otimes \mathbb{1}) \circ\left(\left\langle\varphi_{j i}(u)+\hat{\sigma}(\hat{z}),-\sigma(-)\right\rangle \otimes \mathbb{1}\right) \circ\left(\mathcal{F}^{-1} \otimes \mathbb{1}\right) \circ \eta_{j i}(u)(\hat{z}) \circ\right. \\
& \left.(\mathcal{F} \otimes \mathbb{1}) \circ(\langle\hat{\sigma}(\hat{z}), \sigma(-)\rangle \otimes \mathbb{1}) \circ\left(\mathcal{F}^{-1} \otimes \mathbb{1}\right)\right) \\
= & \operatorname{Ad}\left((\mathcal{F} \otimes \mathbb{1}) \circ\left(\left\langle\varphi_{j i}(u)+\hat{\sigma}(\hat{z}),-\sigma(-)\right\rangle \otimes \mathbb{1}\right)\right. \\
& \left(\lambda_{G / N}\left(-g_{j i}(u)\right) \otimes \mathbb{1}\right) \bar{\zeta}_{j i}(u)\left(-_{-}\right)\left(\varphi_{j i}^{\prime}(u)\left(-_{-}\right) \otimes \mathbb{1}\right) \\
& \left.(\langle\hat{\sigma}(\hat{z}), \sigma(-)\rangle \otimes \mathbb{1}) \circ\left(\mathcal{F}^{-1} \otimes \mathbb{1}\right)\right) \\
= & \operatorname{Ad}\left(( \mathcal { F } \otimes \mathbb { 1 } ) \circ \left(\left\langle\varphi_{j i}(u)+\hat{\sigma}(\hat{z}), \sigma\left(-+g_{j i}(u)-\sigma(-)\right\rangle \otimes \mathbb{1}\right)\right.\right. \\
& \left(\lambda_{G / N}\left(-g_{j i}(u)\right) \otimes \mathbb{1}\right) \bar{\zeta}_{j i}(u)\left(-_{-}\right)\left(\left\langle\varphi_{j i}(u),-\sigma(-)\right\rangle \varphi_{j i}^{\prime}(u)\left(-_{-}\right) \otimes \mathbb{1}\right) \\
& \left.\circ\left(\mathcal{F}^{-1} \otimes \mathbb{1}\right)\right) \\
= & \operatorname{Ad}\left((\mathcal{F} \otimes \mathbb{1}) \circ\left(\left\langle\hat{\sigma}\left(\hat{g}_{j i}(u)+\hat{z}\right),-\sigma(-)\right\rangle \otimes \mathbb{1}\right)\right. \\
& \left.\left(\lambda_{G / N}\left(-g_{j i}(u)\right) \otimes \mathbb{1}\right) \bar{\zeta}_{j i}(u)\left(-{ }_{-}\right)\left(\phi_{j i}(u)(-\sigma(-), 0) \otimes \mathbb{1}\right) \circ\left(\mathcal{F}^{-1} \otimes \mathbb{1}\right)\right) \\
= & (\mathcal{F} \otimes \mathbb{1}) \circ \hat{\zeta}_{j i}(u)(\hat{z}) \circ\left(\mathcal{F}^{-1} \otimes \mathbb{1}\right) .
\end{aligned}
$$


So the the family $\left\{T_{i} f_{i}\right\}$ defines a section $T f \in \Gamma(\widehat{E}, \widehat{F})$, and we have constructed a map $T: C_{\mathcal{C}}(G, \Gamma(E, F)) \rightarrow \Gamma(\widehat{E}, \widehat{F})$ which extends to an isomorphism of $C^{*}$-algebras

$$
\left.T: G \times \alpha_{\alpha} \Gamma(E, F)\right) \rightarrow \Gamma(\widehat{E}, \widehat{F})
$$

Then the relation $T\left(\widehat{\alpha}_{\chi} f\right)=\alpha^{\hat{\rho}} \chi(T f)$ is established by local calculation in the same manner as over the point in equation (46). Thus $T$ is in fact an isomorphism of $C^{*}$-dynamical systems. 


\section{A Some Notation and Basic Lemmata}

\section{A.1 Groups}

Let $G$ be a Hausdorff locally compact abelian group and $N$ some discrete, cocompact subgroup, i.e. $G / N$ is compact.

Lemma A.1 (i) The quotient map $G \rightarrow G / N$ has local sections.

(ii) The quotient map $G \rightarrow G / N$ has a Borel section.

Proof: (i) $N \subset G$ is discrete, i.e. there exists an open neighbourhood $U$ of $0 \in G$ such that $U \cap N=\{0\}$. Let $+: G \times G \rightarrow G$ be the addition. + is continuous so $+^{-1}(U)$ is an open neighbourhood of $(0,0) \in G \times G$. So there is an open neighbourhood $V \subset G$ of $0 \in G$ such that $V \times V \subset+{ }^{-1}(U)$. Let $W:=V \cap(-V)$. Then $W$ is an open neighbourhood of $0 \in G$, and for all $x \in W$ and $n \in N \backslash\{0\}$ the sum $x+n \notin W$, for $x \in W$ implies $-x \in W$ and in case $x+n \in W$ we would find $(x+n)+(-x)=n \in U-$ a contradiction. Therefore $W$ maps injectively to $G / N$, and as the quotient map is open it defines a homoeomorphism from $W$ to its image $W / N$. This defines a local section from $W / N$ to $G$, and using addition in $G / N$ we can move $W / N$ all over $G / N$ to get a local section in the neighbourhood of each point in $G / N$.

(ii) This follows from compactness of $G / N$. For let $s_{m}: U_{m} \rightarrow G, m=1, \ldots, n$ be a family of local sections such that $\bigcup_{m=1}^{n} U_{m}=G / N$, then

$$
\sigma(z):= \begin{cases}s_{1}(z), & \text { if } z \in U_{1}, \\ s_{2}(z), & \text { if } z \in U_{2} \backslash U_{1}, \\ \vdots & \vdots \\ s_{n}(z), & \text { if } z \in U_{n} \backslash \bigcup_{m=1}^{n-1} U_{m}\end{cases}
$$

defines a Borel section.

The dual group of $G$ is $\widehat{G}:=\operatorname{Hom}(G, \mathrm{U}(1))$. With compact-open topology it becomes again a Hausdorff, locally compact group, and if $G$ is second countable, then also $\widehat{G}$ is. For parings of a group and its dual we will use bracket notation $\langle\chi, g\rangle,\langle\alpha, n\rangle, \cdots \in \mathrm{U}(1)$, for $g \in G, \chi \in \widehat{G}, n \in N, \alpha \in \widehat{N}$.

We recall part of the classical duality theorems [Ru]. Pontrjagin Duality states that the canonical map $G$ to $\widehat{\widehat{G}}$ is an isomorphism of topological groups. Moreover, if $N^{\perp}:=\left\{\chi \in \widehat{G}|\chi|_{N}=1\right\}$ is the annihilator of $N$, then there is a canonical isomorphism $\widehat{G / N} \ni \alpha \mapsto(g \mapsto\langle\alpha, g N\rangle) \in N^{\perp}$, and by the same means $\widehat{G} / N^{\perp} \cong \widehat{N}$. Further, the dual group of a discrete group is compact and vice versa, so $N^{\perp} \subset \widehat{G}$ is a discrete cocompact subgroup, thus the situation is completely symmetric under exchange of $N, G$ by $N^{\perp}, \widehat{G}$. Let us denote the integration of a (compactly supported, continuous) function $f: G \rightarrow \mathbb{C}$ against 
the Haar measure of $G$ simply by $\int_{G} f(g) d g$. For the Fourier transform $\hat{f}$ of $f$ we use the convention $\hat{f}(\chi):=\int_{G}\langle\chi, g\rangle f(g) d g, \chi \in \widehat{G}$. It extends to an isomorphism $L^{2}(G) \stackrel{\wedge}{\rightarrow} L^{2}(\widehat{G})$.

\section{A.2 Group and Čech Cohomology}

For a topological $G$-module $M$ let us denote by $C_{\text {cont }}^{k}(G, M)\left(\operatorname{resp} . C_{\text {Bor }}^{k}(G, M)\right.$ ) the continuous (resp. Borel) maps $G^{k} \rightarrow M, k=0,1,2, \ldots\left(C_{?}^{0}(G, M):=M\right)$. They are topological spaces with the compact-open topology. The differential $d: C_{?}^{k}(G, M) \rightarrow C_{?}^{k+1}(G, M)$, given by

$$
\begin{aligned}
d f\left(g_{1}, \ldots, g_{k+1}\right) & :=(-1)^{k+1} f\left(g_{1}, \ldots, g_{k}\right) \\
& +\sum_{i=1}^{k}(-1)^{i} f\left(g_{1}, \ldots, g_{i}+g_{i+1}, \ldots, g_{k+1}\right) \\
& +g_{1} \cdot f\left(g_{2}, \ldots, g_{k+1}\right)
\end{aligned}
$$

makes $C_{?}^{*}(G, M)$ a cochain complex with cohomology groups $H_{?}^{k}(G, M):=$ $Z_{?}^{k}(G, M) / B_{?}^{k}(G, M)$, for the cocycles $Z_{?}^{k}(G, M):=\operatorname{ker}\left(d_{k}\right)$ and the boundaries $B_{?}^{k}(G, M):=\operatorname{im}\left(d_{k-1}\right)$.

Let $U_{\bullet}=\left\{U_{i} \mid i \in I\right\}$ be an open covering of a space $B$. By $U_{i_{0} \ldots i_{n}}$ we denote the intersection $U_{i_{0}} \cap \cdots \cap U_{i_{n}}$. Let $\mathcal{F}$ be any abelian sheaf and let $\check{C}^{k}\left(U_{\bullet}, \mathcal{F}\right):=$ $\prod \mathcal{F}\left(U_{i_{0} \ldots i_{k}}\right)$. The boundary operator $\delta: \check{C}^{k}\left(U_{\bullet}, \mathcal{F}\right) \rightarrow \check{C}^{k+1}\left(U_{\bullet}, \mathcal{F}\right)$ is given by

$$
(\delta \varphi)_{i_{0} \ldots i_{k+1}}:=\varphi_{i_{1} \ldots i_{k+1}}\left|U_{i_{0} \ldots i_{k+1}}-\varphi_{i_{0} i_{2} \ldots i_{k+1}}\right| U_{i_{0} \ldots i_{k+1}}+\cdots+(-1)^{k+1} \varphi_{i_{0} \ldots i_{k}} \mid U_{i_{0} \ldots i_{k+1}}{ }
$$

and we use the standard notation for the cohomology groups $\check{H}^{k}\left(U_{\bullet}, \mathcal{F}\right)$.

We also use the notation $\underline{A}$ to denote the locally constant sheaf of continuous functions to $A$, for any abelian topological group $A$.

\section{A.3 The Unitary and the Projective Unitary Group}

Let $\mathcal{H}$ be some infinite dimensional, separable Hilbert space with unitary group $\mathrm{U}(\mathcal{H})$ which we equip with the strong (or equivalently weak) operator topology. We denote by

$$
\operatorname{Ad}: \mathrm{U}(\mathcal{H}) \rightarrow \mathrm{PU}(\mathcal{H}):=\mathrm{U}(\mathcal{H}) / \mathrm{U}(1)
$$

the quotient map from the unitary onto the projective unitary group, and we endow the latter with the quotient topology.

Let $U \subset \mathrm{U}(\mathcal{H})$ be any subset, e.g. $U=\mathrm{U}(1) \cdot \mathbb{1}$ and let $M$ be some measure space, e.g. $G$ or $G / N$, then we denote by

$$
L^{\infty}(M, U) \subset \mathrm{U}\left(L^{2}(M) \otimes \mathcal{H}\right)
$$


the set of unitary operators which are given by (equivalence classes of) measurable functions $f: M \rightarrow U$ which act as multiplication operators. In particular $L^{\infty}(M, U)$ has the subspace topology of $U\left(L^{2}(M) \otimes \mathcal{H}\right)$ which is usually referred as the weak topology on $L^{\infty}(M, U)$. The image of $L^{\infty}(M, U)$ under Ad $: \mathrm{U}\left(L^{2}(M) \otimes \mathcal{H}\right) \rightarrow \mathrm{PU}\left(L^{2}(M) \otimes \mathcal{H}\right)$ is denoted by $L^{\infty}(M, U)$.

Let us equip the Borel functions $\operatorname{Bor}(G, U)$ with the compact-open topology.

Lemma A.2 The natural map

$$
\operatorname{Bor}(G, U) \rightarrow L^{\infty}(G, U)
$$

is continuous.

Proof : A standard $\varepsilon / 3$-argument. Let $f_{\alpha} \rightarrow f$ be a converging net in $\operatorname{Bor}(G, U)$, i.e. for each compact $K \subset G$ and any $w \in \mathcal{H}$ we have

$$
\left\|f_{\alpha}-f\right\|_{K, w}:=\sup _{g \in K}\left\|f_{\alpha}(g) w-f(g) w\right\|_{\mathcal{H}} \rightarrow 0 .
$$

We have to show that for all $v \in L^{2}(G, \mathcal{H})$

$$
\left\|f_{\alpha} v-f v\right\|_{L^{2}(G, \mathcal{H})} \rightarrow 0 .
$$

Recall that in case of the Haar measure the compactly supported functions $C_{c}(G)$ are dense in $L^{2}(G)$. So for any $v \in L^{2}(G, \mathcal{H})$ and $\varepsilon>0$ there exist compactly supported functions $h_{i} \in C_{\mathcal{c}}(G)$, vectors $w_{j} \in \mathcal{H}$ and numbers $a_{i j} \in \mathbb{C}$ such that $\left\|v-\sum_{i, j=1}^{N} a_{i j} h_{i} \otimes w_{j}\right\|_{L^{2}(G, \mathcal{H})}<\varepsilon / 3$.

Choose $K:=\bigcup_{i=1}^{N}$ supp $h_{i} \subset G$ and $C:=1+\sum_{i, j=1}^{N}\left|a_{i j}\right|^{2} \int_{K}\left|h_{i}(g)\right|^{2} d g>0$. Then there exists an $\alpha_{0}$ such that $\left\|f_{\alpha}-f\right\|_{K, w_{j}}<\frac{\varepsilon}{3 N \sqrt{C}}$, for all $\alpha>\alpha_{0}$ and all $j=1, \ldots, N$.

We can estimate now

$$
\begin{aligned}
&\left\|f_{\alpha} v-f v\right\|_{L^{2}(G, \mathcal{H})} \leq\left\|f_{\alpha}\left(v-\sum_{i, j=1}^{N} a_{i j} h_{i} \otimes w_{j}\right)\right\|_{L^{2}(G, \mathcal{H})}+\left\|\left(f_{\alpha}-f\right)\left(\sum_{i, j=1}^{N} a_{i j} h_{i} \otimes w_{j}\right)\right\|_{L^{2}(G, \mathcal{H})}+\left\|f\left(\sum_{i, j=1}^{N} a_{i j} h_{i} \otimes w_{j}-v\right)\right\|_{L^{2}(G, \mathcal{H})} \\
& \leq \quad\left\|f_{\alpha}\right\|_{\mathrm{Op}} \cdot \varepsilon / 3+\left(\int_{K}\left\|\sum_{i, j=1}^{N} a_{i j} h_{i}(g)\left(f_{\alpha}(g) w_{j}-f(g) w_{j}\right)\right\|_{\mathcal{H}}^{2} d g\right)^{1 / 2}+\|f\|_{\mathrm{OP}} \cdot \varepsilon / 3 \\
& \leq \quad \varepsilon / 3+\left(N^{2} \sum_{i, j=1}^{N}\left|a_{i j}\right|^{2} \int_{K}\left|h_{i}(g)\right|^{2}\left\|\left(f_{\alpha}(g) w_{j}-f(g) w_{j}\right)\right\|_{\mathcal{H}}^{2} d g\right)^{1 / 2}+\varepsilon / 3 \\
& \leq \varepsilon / 3+\left(N^{2} \sum_{i, j=1}^{N}\left|a_{i j}\right|^{2} \int_{K}\left|h_{i}(g)\right|^{2} \frac{\varepsilon^{2}}{9 N^{2} C} d g\right)^{1 / 2}+\varepsilon / 3 \\
& \leq \varepsilon / 3+\sqrt{\varepsilon^{2} / 9}+\varepsilon / 3=\varepsilon,
\end{aligned}
$$


for all $\alpha>\alpha_{0}$.

For PU $(\mathcal{H})$-principal bundles we have the following well-known classification theorem. (See e.g. [Di, Thm. 10.8.4] for the first and of [PR] for the second statement; although therein it is not stated as below, we can carry over the proofs.) To state the theorem we introduce some notation. Let us denote by $\operatorname{Iso}(E)$ the set of isomorphism classes of $\mathrm{PU}(\mathcal{H})$-principal bundles over $E$, and if $P \rightarrow E$ is a $P U(\mathcal{H})$-principal bundle, we denote by $\operatorname{Aut}_{0}(P, E)$ the group of bundle automorphisms (over the identity of $E$ ). There is the subgroup $\operatorname{Null}(P, E) \subset \operatorname{Aut}_{0}(P, E)$ which consists of all null-homotopic bundle automorphisms.

Theorem A.1 Let E be a paracompact Hausdorff space and $P \rightarrow E$ be any fixed $\mathrm{PU}(\mathcal{H})$-principal bundle.

1. $\mathrm{PU}(\mathcal{H})$-bundles over E are classified by the second Čech cohomology with values in the locally constant sheaf of continuous functions to $\mathrm{U}(1)$,

$$
\operatorname{Iso}(E) \cong \check{H}^{2}(E, \underline{U(1)}) \text {. }
$$

2. There is a short exact sequence

$$
0 \rightarrow \operatorname{Null}(P, E) \rightarrow \operatorname{Aut}_{0}(P, E) \rightarrow \check{H}^{1}(E, \mathrm{U}(1)) \rightarrow 0 .
$$

In particular, if $P \rightarrow E$ is the trivial bundle $P=E \times \mathrm{PU}(\mathcal{H})$ we identify $\operatorname{Aut}_{0}(P, E)$ with the continuous functions $C(E, P U(\mathcal{H}))$. If we keep this in mind, a corollary of the classification theorem of bundle automorphisms is the following statement which is sometimes called homotopy commutativity of the projective unitary group.

Corollary A.1 Let E be a paracompact Hausdorff space and $f, g: E \rightarrow \mathrm{PU}(\mathcal{H})$ two continuous functions. Then there extists a continuous function $V: E \rightarrow \mathrm{U}(\mathcal{H})$ such that

$$
f(x) g(x)=g(x) f(x) \operatorname{Ad}(V(x)), \quad x \in E .
$$

Proof : $x \mapsto f(x) g(x)$ and $x \mapsto g(x) f(x)$ define the same Čech class.

We do not give a proof of Theorem A.1, but we remark that it depends heavily on the the fact that the unitary group $\mathrm{U}(\mathcal{H})$ is contractible. For the strong topology on $\mathrm{U}(\mathcal{H})$ this is not difficult to prove and may be found in [Di, 10.8]. We line out the proof for convenience. Assume $\mathcal{H}=L^{2}([0,1])$, then let $\varphi_{t}: L^{2}(0, t) \cong L^{2}(0,1)$ be the isometric isomorphism defined by $\varphi_{t}(f)(x):=$ $\sqrt{t} f(t x)$, for $t>0$. We define $H:[0,1] \times \mathrm{U}\left(L^{2}(0,1)\right) \rightarrow \mathrm{U}\left(L^{2}(0,1)\right)$ by $H(0, U):=\mathbb{1}$ and

$$
(H(t, U)(f))(x):=\left\{\begin{array}{ll}
\left(\varphi_{t}^{-1} \circ U \circ \varphi_{t}\left(\left.f\right|_{(0, t)}\right)\right)(x), & \text { if } 0<x<t, \\
f(x), & \text { if } t<x<1,
\end{array} \text { for } t>0,\right.
$$

then $H$ is a homotopy connecting the identity on $\mathrm{U}(\mathcal{H})$ and the constant function with value $\mathbb{1} \in \mathrm{U}(\mathcal{H})$, thus $H$ is a contraction. 
Remark A.1 For each fixed time slice $t \in[0,1] H(t$, . ) is a group homomorphism. So $\mathrm{U}(\mathcal{H})$ is contractible as group

Remark A.2 Theorem A.1 also holds when we replace the unitary group $\mathrm{U}(\mathcal{H})$ by any contractible, abelian (sub)group $\mathrm{U}^{\mathrm{ab}}(\mathcal{H})$ such that $\mathrm{U}(1) \subset \mathrm{U}^{\mathrm{ab}}(\mathcal{H})$. I.e. the second Čech cohomology $\breve{H}^{2}(E, \mathrm{U}(1))$ also classifies $\mathrm{PU}^{\mathrm{ab}}(\mathcal{H})$-bundles over paracompact Hausdorff spaces $E$ and the first Čech cohomology $\check{H}^{1}(E, \mathrm{U}(1))$ also classifies $\operatorname{PU}^{\mathrm{ab}}(\mathcal{H})$-bundle automorphisms, for $\mathrm{PU}^{\mathrm{ab}}(\mathcal{H}):=\mathrm{U}^{\mathrm{ab}}(\mathcal{H}) / \mathrm{U}(1)$.

The next lemma shows that there exists a appropriate commutative version $\mathrm{U}(1) \rightarrow \mathrm{U}^{\mathrm{ab}}(\mathcal{H}) \rightarrow \mathrm{PU}^{\mathrm{ab}}(\mathcal{H})$ of $\mathrm{U}(1) \rightarrow \mathrm{U}(\mathcal{H}) \rightarrow \mathrm{PU}(\mathcal{H})$.

Lemma A.3 There exists a contractible, commutative subgroup $\mathrm{U}^{\mathrm{ab}}(\mathcal{H}) \subset \mathrm{U}(\mathcal{H})$ such that $\mathrm{U}(1) \cdot \mathbb{1} \subset \mathrm{U}^{\mathrm{ab}}(\mathcal{H})$.

Proof : We let $\mathcal{H}=L^{2}(0,1)$ and $\mathrm{U}^{\mathrm{ab}}(\mathcal{H}):=L^{\infty}([0,1], \mathrm{U}(1))$. It is a commutative subgroup and $\mathrm{U}(1) \cdot \mathbb{1} \subset \mathrm{U}^{\mathrm{ab}}(\mathcal{H})$. The contraction $H$ from above restricts to $h:=\left.H\right|_{[0,1] \times \mathrm{U}^{\mathrm{ab}}(\mathcal{H})}:[0,1] \times \mathrm{U}^{\mathrm{ab}}(\mathcal{H}) \rightarrow \mathrm{U}^{\mathrm{ab}}(\mathcal{H})$ and is given by

$$
h(t, g)(s):=\left\{\begin{array}{ll}
g\left(\frac{1}{t} s\right), & \text { if } s<t \\
1, & \text { if } s>t
\end{array} .\right.
$$

\section{A.4 Crossed Products}

Let $(A, G, \alpha)$ be a $C^{*}$-dynamical system, i.e. $A$ is some $C^{*}$-algebra equipped with a (strongly) continuous action $\alpha: G \rightarrow \operatorname{Aut}(A)$. Let $(\pi, \mathcal{H})$ be some faithful representation of $A$. We embed $C_{c}(G, A)$, the compactly supported continuous functions $G \rightarrow A$, into $\mathcal{L}\left(L^{2}(G, \mathcal{H})\right)$ : define

$$
f \times_{-}: L^{2}(G, \mathcal{H}) \rightarrow L^{2}(G, \mathcal{H})
$$

by

$$
(f \times F)(g):=\int_{G} \pi\left(\alpha_{-g}(f(h))\right) F(g-h) d h,
$$

for $f \in C_{c}(G, A), F \in L^{2}(G, \mathcal{H})$. The adjoint operator of $f \times$ is easily calculated and is $\left(f \times_{-}\right)^{*}=f^{\times} \times{ }_{-}$, wherein $f^{\times}(g):=\alpha_{g}(f(-g))^{*}$. Clearly $f^{\times}$has compact support, and $f^{\times}$is continuous (all $\alpha_{g}$ have norm 1 ). So $C_{c}(G, A)$ is closed under the ${ }^{\times}$-operation. Furthermore one has ${ }^{16} f_{1} \times\left(f_{2} \times F\right)=\left(f_{1} \times f_{2}\right) \times F$, wherein

$$
\left(f_{1} \times f_{2}\right)(g):=\int_{G} f_{1}(h) \alpha_{h}\left(f_{2}(g-h)\right) d h
$$

\footnotetext{
${ }^{16}$ We use the same symbol $\times$ for different maps.
} 
again defines an element of $C_{\mathcal{c}}(G, A)$. Thus $C_{\mathcal{c}}(G, A) \hookrightarrow \mathcal{L}\left(L^{2}(G, \mathcal{H})\right)$ is a *subalgebra. The crossed product of $G$ and $A$ is then defined as the norm completion

$$
G \times{ }_{\alpha} A:=\left({\overline{C_{c}(G, A)}}^{\|\cdot\|}, \times, \times\right) .
$$

(This is in fact well-defined, since the operator norm of $f \times_{-}$is independent of the faithful representation $(\pi, \mathcal{H})$.)

For $\chi \in \widehat{G}, f \in C_{c}(G, A)$ we set $\hat{\alpha}_{\chi}(f)(g):=\langle\chi, g\rangle f(g)$ which extends to a strongly continuous action $\hat{\alpha}: \widehat{G} \rightarrow \operatorname{Aut}\left(G \times{ }_{\alpha} A\right)$, so $\left(G \times_{\alpha} A, \widehat{G}, \hat{\alpha}\right)$ again defines a $C^{*}$-dynamical system. Going once more through the process of building the crossed product gives a $C^{*}$-dynamical system $\left(\widehat{G} \times \hat{\alpha}\left(G \times{ }_{\alpha} A\right), G, \hat{\hat{\alpha}}\right)$, and a key statement in the analysis of crossed products is the following Takai Duality Theorem (see e.g. [Pe1]).

Theorem A.2 There is an isomorphism of $C^{*}$-dynamical systems

$$
\left(\widehat{G} \times \hat{\alpha}\left(G \times{ }_{\alpha} A\right), G, \hat{\hat{\alpha}}\right) \cong\left(A \otimes_{C^{*}} \mathcal{K}\left(L^{2}(G)\right), G, \alpha \otimes \operatorname{Ad} \circ \varrho\right),
$$

for the right regular representation $\varrho: G \rightarrow \mathrm{U}\left(L^{2}(G)\right)$, i.e. $\left(\varrho_{g} f\right)(h):=f(h+g)$ and $\operatorname{Ad}\left(\varrho_{g}\right)(K)=\varrho_{g} K \varrho_{g}^{-1}$, for $K \in \mathcal{K}\left(L^{2}(G)\right)$.

\section{A.5 Some Topology}

We will sometimes use the word space as abbreviation for topological space. Let $X, Y$ be topological spaces. By Bor $(X, Y)$ we denote the set of Borel functions from $X$ to $Y$. We endow this space with the compact-open topology, i.e. we define a basis of the topology by all sets of the form $U_{K, V}:=\{f: X \rightarrow$ $Y \mid f(K) \subset V\}$ for compact $K \subset X$ and open $V \subset Y$. The subspace of continuous maps will be denoted by $\operatorname{Map}(X, Y) \subset \operatorname{Bor}(X, Y)$. We use the notation $C(X, Y)$ for the continuous functions if we do not want to specify the topology on it, or in case $Y$ is a normed space, then we put the supremums norm on $C(X, Y)$. Recall the exponential law [Sch] for Map $(., .$.$) .$

Lemma A.4 Let $X, Y, Z$ be topological spaces. Assume $X$ and $Y$ to be Hausdorff and $Y$ locally compact. Then we have a homoeomorphism

$$
\begin{aligned}
\operatorname{Map}(X \times Y, Z) & \cong \operatorname{Map}(X, \operatorname{Map}(Y, Z)) \\
f & \mapsto(x \mapsto f(x,-)) .
\end{aligned}
$$

Let $E \rightarrow B$ be a surjective fibration and assume $Y$ is locally compact and Hausdorff. By use of the exponential law it is then immediate that $\operatorname{Map}(Y, E) \rightarrow$ $\operatorname{Map}(Y, B)$ still has the homotopy lifting property with respect to all Hausdorff spaces; we denote this property by $T_{2} H L P$. For contractible $E$ the map $\operatorname{Map}(Y, E) \rightarrow \operatorname{Map}_{0}(Y, B):=\{f \simeq$ const. $\}$ becomes a surjection having the $T_{2} H L P$. 
We now restrict ourselves to the particular case of $E=\mathrm{U}(\mathcal{H}), B=\mathrm{PU}(\mathcal{H})$. Since $\mathrm{U}(\mathcal{H})$ is Polish $[\mathrm{Ke}]$ the quotient map $\mathrm{U}(\mathcal{H}) \rightarrow \mathrm{PU}(\mathcal{H})$ admits a Borel section $s: \mathrm{PU}(\mathcal{H}) \rightarrow \mathrm{U}(\mathcal{H})[\mathrm{Ke}$, Thm. 12.7].

Let $f \in \operatorname{Map}(Y, \operatorname{PU}(\mathcal{H}))$ be a map not homotopic to a constant map. The Borel function $s_{*} f=s \circ f: Y \rightarrow \mathrm{U}(\mathcal{H})$ gives rise to a bijection of sets

$$
\begin{aligned}
\operatorname{Map}(Y, \mathrm{U}(\mathcal{H})) & \rightarrow \operatorname{Map}(Y, \mathrm{U}(\mathcal{H})) \cdot s_{*} f . \\
g & \mapsto g \cdot s_{*} f
\end{aligned}
$$

We turn this map into a homoeomorphism by defining the topology of the right hand side to be the image of the topology of the left hand side. Then

$$
\operatorname{Map}(Y, \mathrm{U}(\mathcal{H})) \cdot s_{*} f \rightarrow \operatorname{Map}_{0}(Y, \mathrm{PU}(\mathcal{H})) \cdot f .
$$

as well as the assembled map

$$
\coprod_{f \in \pi}\left(\operatorname{Map}(Y, \mathrm{U}(\mathcal{H})) \cdot s_{*} f\right) \rightarrow \coprod_{f \in \pi}\left(\operatorname{Map}_{0}(Y, \operatorname{PU}(\mathcal{H})) \cdot f\right)
$$

becomes a surjection satisfying the $T_{2} H L P$. Here $\pi:=\{f: Y \rightarrow \operatorname{PU}(\mathcal{H})\}$ is a set of representatives of the first homotopy group $\pi_{0}(\operatorname{Map}(Y, P U(\mathcal{H})))$. If $Y$ is compact $\operatorname{Map}_{0}(Y, \operatorname{PU}(\mathcal{H})) \subset \operatorname{Map}(Y, \operatorname{PU}(\mathcal{H}))$ is open, i.e.

$$
\operatorname{Map}(Y, \operatorname{PU}(\mathcal{H}))=\coprod_{f \in \pi}\left(\operatorname{Map}_{0}(Y, \operatorname{PU}(\mathcal{H})) \cdot f\right) .
$$

To consider non compact $Y$ the following easy statement is helpful.

Lemma A.5 Let $Z$ be any (pointed) space, and let $Z_{z}$ denote the path-connected component of $z \in Z$. Then

$$
\coprod_{[z] \in \pi_{0}(Z)} Z_{z} \rightarrow \bigcup_{[z] \in \pi_{0}(Z)} Z_{z}=Z
$$

is a fibration.

Proof : Give any test space $X$ and a diagram

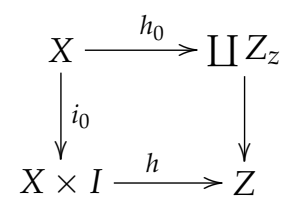

we define the (unique) homotopy $\tilde{h}: X \times I \rightarrow \amalg Z_{z}$ that fits into the above diagram simply by $\tilde{h}(x, t):=h(x, t)$. One has to check that $\tilde{h}$ is continuous. For this it is sufficient to show that $\tilde{h}^{-1}\left(Z_{z}\right)$ is open. Since $h$ maps path-connected components into path-connected components we have $h^{-1}\left(Z_{z}\right)=h_{0}^{-1}\left(Z_{z}\right) \times I$. Since $h_{0}^{-1}\left(Z_{z}\right) \subset X$ is open, it follows that $\tilde{h}^{-1}\left(Z_{z}\right)=h^{-1}\left(Z_{z}\right)=h_{0}^{-1}\left(Z_{z}\right) \times I \subset$ $X \times I$ is open. 
This lemma illustrates that

$$
\coprod_{f \in \pi}\left(\operatorname{Map}(Y, \mathrm{U}(\mathcal{H})) \cdot s_{*} f\right) \rightarrow \operatorname{Map}(Y, \operatorname{PU}(\mathcal{H}))
$$

is surjective and satisfies the $T_{2} H L P$ for all locally compact Hausdorff spaces $Y$, e.g. $Y=G \times G / N$. This implies the next

Corollary A.2 Let $U$ be a contractible Hausdorff space, and let $\mu: U \rightarrow \operatorname{Map}(G \times$ $G / N, \operatorname{PU}(\mathcal{H}))$ be continuous. Then there exists a continuous lift $\bar{\mu}: U \rightarrow \operatorname{Bor}(G \times$ $G / N, \mathrm{U}(\mathcal{H}))$ such that $\operatorname{Ad}_{*} \circ \bar{\mu}=\mu$, i.e. $\operatorname{Ad} \circ \bar{\mu}(u)=\mu(u)$ for all $u \in U$.

Proof : (52) has the $T_{2} H L P$ and $U$ is contractible and Hausdorff.

For the remainder of this paragraph we stick to compact $Y$, e.g. $Y=G / N$. We shall consider the map $\operatorname{Ad}_{*}: \operatorname{Bor}(Y, \mathrm{U}(\mathcal{H})) \rightarrow \operatorname{Bor}(Y, \mathrm{PU}(\mathcal{H}))$.

Lemma A.6 $\quad \mathrm{Ad}_{*}$ is continuous and open.

Proof : Note first that $\mathrm{Ad}: \mathrm{U}(\mathcal{H}) \rightarrow \mathrm{PU}(\mathcal{H})$ is continuous and open.

Continuity: Let $U_{K, V}:=\{g \mid g(K) \subset V, K \subset Y$ compact, $V \subset \mathrm{PU}(\mathcal{H})$ open $\}$. Then $\operatorname{Ad}_{*}^{-1}\left(U_{K, V}\right)=\left\{f \mid f(K) \subset \operatorname{Ad}^{-1}(V)\right\}$ is open in $\operatorname{Bor}(Y, U(\mathcal{H}))$.

Openness: Let $U_{K, W}:=\{f \mid f(K) \subset W, K \subset Y$ compact, $W \subset \mathrm{U}(\mathcal{H})$ open $\}$. Then the inclusion $\operatorname{Ad}_{*}\left(U_{K, W}\right) \subset\{g \mid g(K) \subset \operatorname{Ad}(W)\}$ is obvious; we show equality. To do so it is sufficient to construct a Borel section $s$ of $\mathrm{U}(\mathcal{H}) \rightarrow$ $\mathrm{PU}(\mathcal{H})$ such that $s(\operatorname{Ad}(W)) \subset W . \mathrm{PU}(\mathcal{H})$ is separable. Take a countable dense set $\left\{x_{i}\right\}_{i} \in \mathbb{N}$ and a local trivialisation $U_{0} \subset \mathrm{PU}(\mathcal{H})$ of $\mathrm{U}(\mathcal{H}) \rightarrow \mathrm{PU}(\mathcal{H})$. Let $U_{i}:=U_{0} x_{i} \subset \operatorname{PU}(\mathcal{H})$ and $V_{i}:=\operatorname{Ad}(W) \cap U_{i}$, so $\bigcup_{i} V_{i}=\operatorname{Ad}(W)$. It suffices to construct Borel sections $s_{i}: V_{i} \rightarrow W$ and puzzling them together by

$$
s(z):=\left\{\begin{array}{ll}
s_{1}(z), & \text { if } z \in V_{1}, \\
s_{2}(z), & \text { if } z \in V_{2} \backslash V_{1}, \\
\vdots & \vdots \\
s_{n}(z), & \text { if } z \in V_{n} \backslash \bigcup_{m=1}^{n-1} V_{m} \\
\vdots & \vdots
\end{array},\right.
$$

then $s$ is Borel, since we put together a countable family. The sections $s_{i}: V_{i} \rightarrow$ $W$ may be obtained by similar manners: Let $h_{i}: \operatorname{Ad}^{-1}\left(V_{i}\right) \rightarrow V_{i} \times \mathrm{U}(1)$ be a trivialisation, and let $W_{i}:=h_{i}\left(\operatorname{Ad}^{-1}\left(V_{i}\right) \cap W\right)$. Then $W_{i} \subset V_{i} \times \mathrm{U}(1)$ is open. Thus for each $l \in L:=\left\{l \in \mathbb{N} \mid x_{l} \in V_{i}\right\}$ there is $\varphi_{l} \in \mathrm{U}(1)$ and an open neighbourhood $V_{i}^{l} \ni x_{l}$ such that $V_{i}^{l} \times\left\{\varphi_{l}\right\} \subset W_{i}$. We define

$$
s_{i}(z):=\left\{\begin{array}{ll}
h_{i}^{-1}\left(z, \varphi_{l_{1}}\right), & \text { if } z \in V_{i}^{l_{1}}, \\
h_{i}^{-1}\left(z, \varphi_{l_{2}}\right), & \text { if } z \in V_{i}^{l_{1}} \backslash V_{i}^{l_{2}}, \\
\vdots & \vdots \\
h_{i}^{-1}\left(z, \varphi_{l_{n}}\right), & \text { if } z \in V_{i}^{l_{n}} \backslash \bigcup_{m=1}^{n-1} V_{i}^{l_{m}} \\
\vdots & \vdots
\end{array},\right.
$$


for a counting $l_{1}, l_{2}, \ldots$ of $L$. This completes the proof.

Lemma A.7 If $Y$ is compact then $\operatorname{Ad}_{*}: \operatorname{Bor}(Y, U(\mathcal{H})) \rightarrow \operatorname{Bor}(Y, \operatorname{PU}(\mathcal{H}))$ is a locally trivial principal fibre bundle with structure group $\operatorname{Bor}(Y, \mathrm{U}(1))$.

Proof : Let $t: V \rightarrow \mathrm{U}(\mathcal{H})$ be a local section of $\mathrm{U}(\mathcal{H}) \rightarrow \mathrm{PU}(\mathcal{H})$ for some open $V \subset \operatorname{PU}(\mathcal{H})$. Then $t_{*}: U_{V} \ni f \mapsto t \circ f \in \operatorname{Bor}(Y, \mathrm{U}(\mathcal{H}))$ is a local section on $U_{V}:=\{f: Y \rightarrow \operatorname{PU}(\mathcal{H}) \mid f(Y) \subset V\}$ which is open, since $Y$ is compact. We can cover the whole of $\operatorname{Bor}(Y, \mathrm{PU}(\mathcal{H}))$ by translates of $U_{V}$ under the action of $\operatorname{Bor}(Y, \mathrm{PU}(\mathcal{H}))$ on itself. The lemma will be proven if we can show that $\operatorname{Bor}(Y, \mathrm{PU}(\mathcal{H}))$ and $\operatorname{Bor}(Y, \mathrm{U}(\mathcal{H})) / \operatorname{Bor}(Y, \mathrm{U}(1))$ are homoeomorphic. Take a Borel section $\sigma: \mathrm{PU}(\mathcal{H}) \rightarrow \mathrm{U}(\mathcal{H})$. Then

$$
\begin{aligned}
\sigma_{*}: \operatorname{Bor}(Y, \operatorname{PU}(\mathcal{H})) & \rightarrow \operatorname{Bor}(Y, \mathrm{U}(\mathcal{H})) / \operatorname{Bor}(Y, \mathrm{U}(1)) \\
f & \mapsto[\sigma \circ f]
\end{aligned}
$$

is easily seen to be a bijection such that

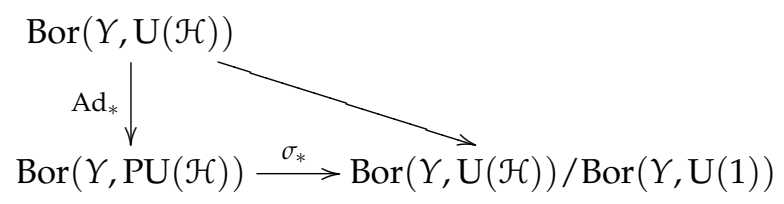

commutes. It follows that $\sigma_{*}$ is a homoeomorphism, for the quotient map and $\mathrm{Ad}_{*}$ are both continuous and open.

We will use the above lemma in combination with the next.

Lemma A.8 Let $P \rightarrow M$ be a locally trivial fibre bundle, and B a paracompact space. Then for each covering $\left\{U_{i} \mid i \in I\right\}$ of $B$ and maps $\zeta_{i j}: U_{i} \cap U_{j} \rightarrow M$, there exists a refinement $\left\{V_{i x} \mid i x \in I \times B\right\}\left(V_{i x} \subset U_{i}\right)$ and continuous $\bar{\zeta}_{i x, j y}: V_{i x} \cap V_{j y} \rightarrow P$ such that the diagram

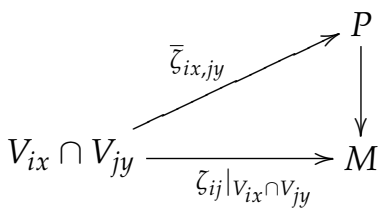

commutes.

Proof : Without restriction we can assume that the covering $\left\{U_{i}\right\}_{i \in I}$ is locally finite, so for each $x \in X I_{x}:=\left\{k \in I \mid\{x\} \cap U_{k} \neq \varnothing\right\}$ is finite. If $x \notin U_{i}$, we define $V_{i x}:=\varnothing$. If $x \in U_{i}$, then $i \in I_{x}$. For each $\zeta_{i k}(x), k \in I_{x}$, choose a local trivialisation $M_{i, k, x} \ni \zeta_{i k}(x)$ of $P \rightarrow M$. Then $V_{i x}:=\bigcap_{k \in I_{x}} \zeta_{i k}^{-1}\left(M_{i, k, x}\right) \subset U_{i}$ is open. By construction the image of $\left.\zeta_{i j}\right|_{V_{i x} \cap V_{j y}}$ is contained in $\bigcap_{k \in I_{x}} M_{i, k, x}$, and we can compose $\zeta_{i j} \mid V_{i x} \cap V_{j y}$ with any local section, say $M_{i, i, x} \rightarrow P$, to define $\bar{\zeta}_{i x, j y}$. 


\section{Index}

\begin{tabular}{|c|c|c|c|}
\hline Symbol & Page & $g_{j i}$ & 19 \\
\hline$\langle.,\rangle$. & 88 & $\hat{g}_{j i}$ & 39 \\
\hline$[.,]$. & 19,76 & $\hat{g}_{j i}$ & 66 \\
\hline & & $I$ & 18 \\
\hline $\mathcal{A}$ & 18 & $\kappa$ & 13 \\
\hline $\mathcal{A}_{1}$ & 19 & $\kappa_{i}$ & 33 \\
\hline$A_{j i}$ & 51 & $K_{i}$ & 53 \\
\hline Ad & 89 & $\kappa_{i}^{u}, \kappa_{i}^{\sigma}$ & 50 \\
\hline$\alpha_{j i}$ & 51 & & 35 \\
\hline$\alpha^{\rho}$ & 79 & $\overline{\hat{\kappa}}^{\tilde{\sigma}}$ & 36 \\
\hline$\alpha^{\mu}$ & 80 & $\kappa^{\text {top }}$ & 47 \\
\hline Aut, Aut $_{0}$, Aut $_{1}$ & 17 & $\kappa_{i}^{\text {top }}$ & 48 \\
\hline$B$ & 15 & $\mathcal{K}(\mathcal{H})$ & ?? \\
\hline $\mathcal{B} \mathcal{A}_{1}$ & 19 & $L^{\infty}$ & 89 \\
\hline$\beta_{j i}$ & 51 & $l_{i}, l_{i}^{0}, \lambda_{i}$ & 60 \\
\hline$\delta_{g}$ & 20 & $\lambda_{G}$ & 28 \\
\hline Bor & 93 & $\lambda_{G / N}$ & 41 \\
\hline$c_{i}$ & 26 & $m_{i}$ & 56 \\
\hline$c^{\tau}$ & 26 & Map & 93 \\
\hline$\chi, \chi_{j i}$ & 56 & $\mu_{i}$ & 24 \\
\hline Dyn & 28 & $\bar{\mu}_{i}$ & 30 \\
\hline Dyn & 31 & $\hat{\mu}_{i}$ & 43 \\
\hline Dyn $^{+}$ & 38 & $\mu_{i}^{\mathrm{dyn}}$ & 61 \\
\hline$\delta$ & 75 & $N$ & 15 \\
\hline$\delta^{\mathrm{s}}, \delta^{\mathrm{as}}, \delta^{\mathrm{im}}$ & 59 & $N^{\perp}$ & 88 \\
\hline E & 15 & $\omega, \omega_{i}$ & 30 \\
\hline$\widehat{E}$ & 31,43 & $P,(P, E)$ & 15 \\
\hline$E_{\mathrm{dyn}}$ & 61 & $\mathcal{P}$ & 59 \\
\hline $\mathcal{E} \mathcal{A}_{1}$ & 19 & $\widehat{P}$ & 31,43 \\
\hline$F$ & 15 & $P_{\text {top }}$ & 47 \\
\hline $\mathcal{F}$ & 46,80 & $P_{\text {dyn }}$ & 61 \\
\hline$\hat{\mathcal{F}}$ & 64 & $P_{\mathcal{H}}$ & 16 \\
\hline$f^{\bar{\mu}}$ & 82 & Par & 16 \\
\hline$G, G / N$ & 15 & $\widehat{\mathrm{Par}}$ & 31 \\
\hline$\widehat{G}, \widehat{G} / N^{\perp}$ & 88 & $\mathrm{PU}(\mathcal{H})$ & 89 \\
\hline$\Gamma, \Gamma_{0}$ & 15,79 & $\pi$ & 32 \\
\hline$\gamma_{j i}$ & 92 & $\varphi, \varphi_{k j i}$ & 53 \\
\hline & & $\varphi^{*} P$ & 15 \\
\hline
\end{tabular}




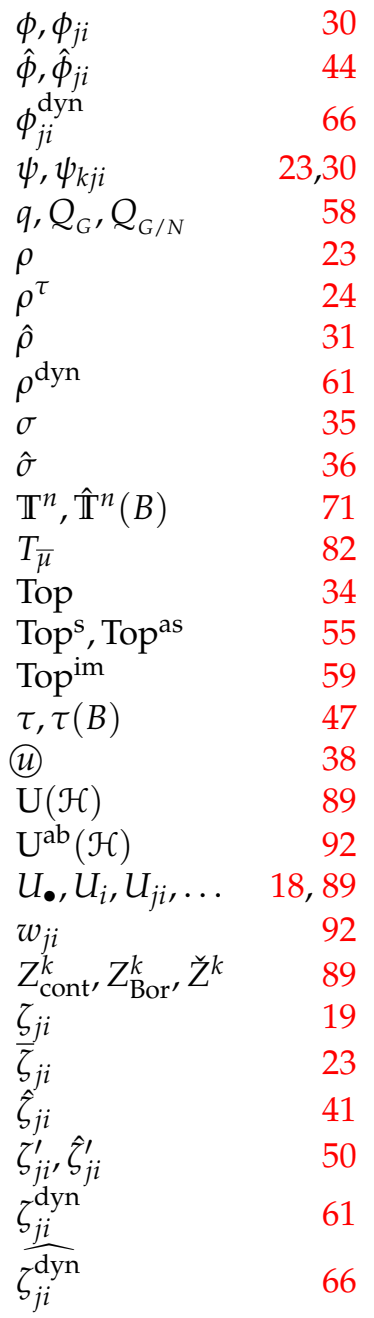




\section{References}

[BEM] P. Bouwknegt, J. Evslin V. Mathai: On the Topology and H-Flux of TDual Manifolds; arXiv: hep-th/0312052

[BHM1] P. Bouwknegt, K. Hannabus, V. Mathai: T-Duality for Principal Torus Bundles, JHEP 0403 (2004) 018; arXiv: hep-th/0312284

[BHM2] P. Bouwknegt, K. Hannabus, V. Mathai: Nonassociative tori and applications to T-duality, Commun.Math.Phys. 264 (2006), 41-6; arXiv: hepth/0412092

[BM] P. Bouwknegt, V. Mathai: D-branes, B-fields and twisted K-theory, J. High Energy Phys. 3 (2000), (7); arXiv: hep-th/0002023.

[BS] U. Bunke, Th. Schick: On the topology of T-duality, Rev. in Math. Phys., vol 17 no. 1, Feb 2005, pp. 77-112; arXiv: math.GT/0501487

[BRS] U. Bunke, Ph. Rumpf, Th. Schick: The topology of T-duality for $T^{n}$ bundels, Rev. in Math. Phys., Vol. 18, No. 10 (2006), pp. 1103-1154; arXiv: math.GT/0501487

[BSST] U. Bunke, Th. Schick, M. Spitzweck, A. Thom: Duality for topological abelian group stacks and T-duality, arXive: math/0701428

[CKRW] D. Crocker, A. Kumjian, I. Raeburn, D. P. Williams: An Equivariant Brauer Group and Actions of Groups on $C^{*}$-Algebras, arXiv: functan/9412002

[DD] J. Dixmier, Douady: Champes continus d'espace hilbertien et de $C^{*}$ algebres, Bulletin de la societe mathematique de france, t. 91 (1963) pp. $227-284$

[Di] J. Dixmier: $C^{*}$-Algebras, North-Holland, Amsterdam, 1977

[ER] S. Echterhoff, J. Rosenberg: Fine structure of the Mackey machine for actions of abelian groups with constant Mackey obstruction, Pacific J. Math. 170(1), pp. 17-52, 1995

[FP] R. Fritsch, R. A. Piccinini: Cellular structures in topology, Cambridge Univ. Press, 1990

[Ke] A.S. Kechris: Classical Descriptive Set Theory, Springer, NY, 1995

[Mo] C. C. Moore: Group Extensions and Cohomology for Locally Compact Groups III, Trans. of Amer. Math. Soc., Vol. 221, No. 1 (July 1976), pp. 1-33

[MR] V. Mathai, J. Rosenberg: T-duality for torus bundles with $H$-flux via noncommutative topology, II: the high-dimensional case and the T-duality group, Adv.Theor.Math.Phys. 10 (2006) 123-158; arXiv: hep-th/0508084 
[OR] D. Olsen, I. Raeburn: Poinwise Unitary Automorphism Groups, J. of Funct. Anal., 93, pp. 278-309, 1990

[Pe1] G. K. Pederson: $C^{*}$-Algebras and their Automorphism Groups, Academic Press, 1979

[Pe2] G. K. Pederson: Crossed products by locally unitary automorphism groups and principal bundles, J. Operator Theory 11, 215-141, 1984

[Po] J. Polchinski: String Theory, Cambridge University Press, 2005

[PR] J. Phillips, I. Raeburn: Automorphisms of $C^{*}$-algebras and Second Čech Cohomology, Ind. Univ. Math. Journal, Vol. 29, 6, 1980

[RR] I. Raeburn, J. M. Rosenberg: Crossed Products of Continuous Trace $C^{*}$ Algebras by Smooth Actions, Trans. of Amer. Math. Soc., Vol. 305, No. 1, pp. $1-45,1988$

[RW] I. Raeburn, D. Williams: Pull backs of $C^{*}$-algebras and crossed products by certain diagonal actions, Trans. of Amer. Soc. 287, 755-777, 1985

[Ru] W. Rudin: Fourier Analysis on Groups, Wiley Classics Library Edition, 1990

[Sch] http://www.math.uni-bonn.de/people/schwede/Lehre/Skripte/AlgTopII_10.pdf 